\title{
"A DAY IN THE LIFE OF THE JOS" THE DESIGN OF AN EDUCATIONAL GAME ON PRIVACY
}

\author{
by \\ Christine Mekhail

\begin{abstract}
A thesis submitted to the Faculty of Graduate and Postdoctoral Affairs

in partial fulfillment of

the requirements for the degree of

MASTER OF ARTS
\end{abstract} \\ School of Computer Science \\ at \\ CARLETON UNIVERSITY
}

Ottawa, Ontario

September, 2016

(c) Copyright by Christine Mekhail, 2016 


\begin{abstract}
The need to educate children on protecting their online privacy and security is pressing, as more children are going online unsupervised. This thesis examines how to make privacy and security education relevant to children's everyday life. We have partnered with MediaSmarts to create a new interactive educational game. As a starting point, we conducted a user study of an existing game, then we designed, prototyped, and evaluated a new game with children. In our new "A Day in the Life of the Jos" (ADITL) game, players follow Jo and Josie, a brother and sister, through their day and help them make positive privacy and security-related choices. Players then witness the outcomes of their decisions on the Jos' lives as they unfold within the game. Evaluation results show that participants found ADITL appealing and that adding gamification elements encouraged engagement with the educational material and promoted interaction with the lessons.
\end{abstract}




\section{Acknowledgements}

First I would like to thank to my supervisor Sonia Chiasson. Without her dedication to her students, support, and hard work this thesis would not have come to fruition. I will always cherish the support, insight, guidance, and the amount of work you have put towards the completion of my thesis, and my Masters journey. Thank you for being a kind human being, before being an excellent, wise guide and supervisor. I would also like to thank Professor Robert Biddle for his guidance in giving his expertise advice during my thesis research phase and through out my Masters courses. Professor Biddle you always amaze me with your wealth of knowledge and insight. You add years of knowledge to ours by freely sharing your years of experience and knowledge with us students. I also would like to thank my professors who helped increase my learning in the various HCI subjects through out my Masters journey.

Many thanks goes to my thesis committee members, Robert Biddle, and Kasia Muldner who provided valuable insight and constructive feedback. Special thanks goes to MediaSmarts team and their excellent guidance and prompt feedback during working on the game redesign. Thanks for providing me the opportunity to work on an area of my passion. Thanks to MITACS for funding my MediaSmarts internship. Thanks to the NSERC CREATE CLUE program for funding my second internship position, and special thanks to Professors Anthony Whitehead and Audrey Girouard who helped me secure the internship position.

Finally, thanks to my husband Sherif, who without his help, vision, and support, I would not have been able to complete this journey. Thanks for the many nights and weekends which you took care of our son, so that I can finish working on this thesis. Your selfless love is what keeps me going and challenging all negative beliefs I might have. 


\section{Table of Contents}

Abstract $\quad$ ii

Acknowledgements $\quad$ iii

List of Tables viii

List of Figures $\quad$ ix

Chapter 1 Introduction 1

1.1 Motivation . . . . . . . . . . . . . . . . . 1

1.2 Research Challenge . . . . . . . . . . . . . . . . . . 2

1.3 Contributions . . . . . . . . . . . . . . . . . . 2

1.4 Thesis Outline . . . . . . . . . . . . . . . . . 3

$\begin{array}{lll}\text { Chapter } 2 \text { Background } & 4\end{array}$

2.1 Children's Developmental Characteristics . . . . . . . . . . . . . 4

2.1.1 Cognitive and physical development . . . . . . . . . . . . . . 4

2.1.2 Social and emotional development . . . . . . . . . . . 5

2.2 Children and Media . . . . . . . . . . . . . . . . . 6

2.2.1 Use of social media . . . . . . . . . . . . . . . . 6

2.2.2 Use of mobile devices . . . . . . . . . . . . . . . 6

2.3 Technology in the Classroom . . . . . . . . . . . . . 6

2.4 Educational Games . . . . . . . . . . . . . . . . . . . 7

2.4.1 Games in the classroom . . . . . . . . . . . . 8

2.5 Security Education . . . . . . . . . . . . . . . . 9

2.5.1 Comics to teach security . . . . . . . . . . . . . 10

2.5.2 Security games . . . . . . . . . . . . . 10

2.6 Design Strategies for Games . . . . . . . . . . . . . . . . . . . . . 12

2.6.1 General strategies . . . . . . . . . . . . . . . 12 
2.6 .2 Learning strategies . . . . . . . . . . . . . . . . . . 13

2.7 Summary . . . . . . . . . . . . . . . . . . . 13

Chapter 3 Initial User Study of Existing Game "Jo Cool or Jo Fool" 14

3.1 Details of JCJF . . . . . . . . . . . . . . . . . . . . . . 14

3.2 Research Topics . . . . . . . . . . . . . . . . . . . . . . . . 18

3.3 Methodology . . . . . . . . . . . . . . . . . . . . . . . . 18

3.3 .1 Ethics . . . . . . . . . . . . . . . . . . 18

3.3.2 Recruitment and Sampling . . . . . . . . . . . . . . . . 19

3.3.3 Procedure . . . . . . . . . . . . . . . . . . . . . . . . . . . . 19

3.4 Results . . . . . . . . . . . . . . . . . . . . . . . . 21

3.4.1 Device Usage and Context . . . . . . . . . . . . . . . . 21

3.4 .2 Pre- and Post-session Interview _ . . . . . . . . . . . . 22

3.4.3 Pre- and Post-scenario Questionnaires . . . . . . . . . . . 24

3.4.4 Reading Times . . . . . . . . . . . . . . . . 25

$3.4 .5 \quad$ User Feedback . . . . . . . . . . . . . . . . . . . . . . . 28

3.4 .6 Eye Tracking Data Analysis . . . . . . . . . . . . . . . . 32

3.4.7 User Feedback Questionnaire . . . . . . . . . . . . . . 39

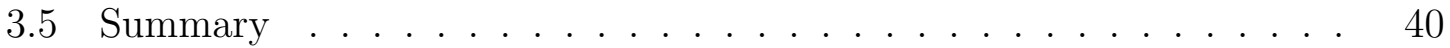

Chapter 4 Design of the new game: "A Day in The Life of the Jos" 42

4.1 The ADDIE instructional design model . . . . . . . . . . . . . 42

4.2 Analysis Phase . . . . . . . . . . . . . . . . . 44

4.2 .1 Intended audience . . . . . . . . . . . . . . . . . 44

4.2 .2 Educational goals . . . . . . . . . . . . . . . . 44

4.2 .3 Entertainment goals . . . . . . . . . . . . . . . . 46

4.2 .4 Conversation-provoking goals $\ldots \ldots \ldots \ldots$

4.3 Design Phase . . . . . . . . . . . . . . . . . . . . . . . 47

4.3 .1 Game topics . . . . . . . . . . . . . . . . . 47

4.3 .2 ADITL overview . . . . . . . . . . . . . . 48

4.3 .3 Game mechanics . . . . . . . . . . . . . . . . . . . . . . 49 
4.4 Development phase: First ADITL prototype . . . . . . . . . . 55

4.4 .1 Visual design . . . . . . . . . . . . . . . . 55

$4.4 .2 \quad$ D1 storyline . . . . . . . . . . . . . . . . . . 57

4.5 Evaluation Phase 1: Pilot Study . . . . . . . . . . . . . . 60

4.5 .1 Procedure . . . . . . . . . . . . . . . . . . . 61

$4.5 .2 \quad$ Data analysis . . . . . . . . . . . . . . . . . 62

4.5 .3 Feedback on Visual Design . . . . . . . . . . . . . . . 62

4.5.4 Feedback on Game Design . . . . . . . . . . . . . . . . 64

4.6 Implementation Phase: Second ADITL Prototype . . . . . . . . . 65

Chapter 5 Evaluation of the new game: "A Day in The Life of the $\begin{array}{ll}\text { Jos" } & 67\end{array}$

5.1 Methodology . . . . . . . . . . . . . . . . . 67

5.1 .1 Demographics . . . . . . . . . . . . . . . . . 67

5.1 .2 Recruitment and Sampling . . . . . . . . . . . . . . 67

5.1 .3 Procedure . . . . . . . . . . . . . . . . . . 68

5.2 Results . . . . . . . . . . . . . . . . . . . . . . . . . 69

5.2.1 Pre- and Post-game-day Interviews _ . . . . . . . . . . . 70

5.2 .2 Game Choices . . . . . . . . . . . . . . . . . . . . . . . 74

5.2 .3 Reading Times . . . . . . . . . . . . . . . . 78

5.2.4 User Feedback Questionnaire . . . . . . . . . . . . 78

5.2.5 Eye Tracking Data Analysis _ . . . . . . . . . . . . 80

5.2 .6 Qualitative Feedback . . . . . . . . . . . . . . 85

5.2 .7 General Observations . . . . . . . . . . . . . . . . . . . 90

5.3 Comparison with old game . . . . . . . . . . . . . . . 91

5.3 .1 Visual appeal . . . . . . . . . . . . . . . . . . 91

5.3 .2 Game topics . . . . . . . . . . . . . . . . . . . 91

5.3 .3 Game elements . . . . . . . . . . . . . . . . . . . . . . 92

5.3 .4 Reading Times . . . . . . . . . . . . . . . . . . . . . 93

5.3.5 User feedback questionnaire . . . . . . . . . . . . . . . 93 
5.3.6 Amount of learning . . . . . . . . . . . . . . . . . . . 94

$\begin{array}{lll}\text { Chapter } 6 & \text { Discussion and Conclusion } & 95\end{array}$

6.1 Discussion . . . . . . . . . . . . . . . . . . . . . . 95

6.1.1 Children as study participants . . . . . . . . . . . 95

6.1.2 Children's Interaction With Games . . . . . . . . . . . . . . 97

6.2 Recommendations . . . . . . . . . . . . . . . . . . . . . 99

6.3 Limitations and Future Work . . . . . . . . . . . . . . . . . . . 99

6.4 Conclusion . . . . . . . . . . . . . . . . . . . . 100

$\begin{array}{ll}\text { Bibliography } & 102\end{array}$

$\begin{array}{ll}\text { Appendix A Appendix } & 110\end{array}$

A.1 JCJF detailed game scenarios . . . . . . . . . . . . . . . . 110

A.2 Parental/Legal Guardian Permission for Children Participation in Re-

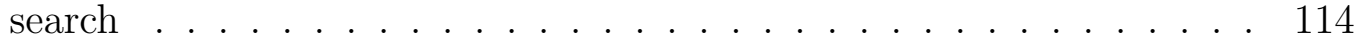

A.3 Verbal Informed Assent Script for the Children . . . . . . . . . . . . 117

A.4 Debriefing Form . . . . . . . . . . . . . . . . . . . . . . . . . . 118

A.5 User Feedback Questionnaire . . . . . . . . . . . . . . . . . . 120

A.6 Pre- and Post-game-day Interviews . . . . . . . . . . . . . . . 122

A.7 D1-Cyberbullyng Day full script: . . . . . . . . . . . . . . . . 123

A.8 25 game scenarios across the five topics . . . . . . . . . . . 128 


\section{List of Tables}

Table 3.1 Number of views for each scenario. *For S2 and S4, we lost the audio and eye-tracking video recording of one participant due to a technical error, leaving 2 full data sets and one partial data set for these two scenarios. . . . . . . . . . . . . . . . 21

Table 3.2 Participants' device usage, main uses and favourite sites. . . . . 23

Table 3.3 Participants' password practices, and their understanding of online privacy. ${ }^{*}$ Missing data for P5 due to technical error. . . . .

Table 4.1 The number of scenarios tested per participant. . . . . . . . . 63

Table 4.2 Participants' preferred character, calendar, and background styles from among the three concepts. . . . . . . . . . . . . 63

Table 5.1 Total number of participants who viewed each scenario. . . . . 70 


\section{List of Figures}

Figure 3.1 (a) JCJF landing page. (b) Main navigation page. (c) through

(f) show the sequence of screens of Sc1: Splurge Cola where Josie chooses to provide her personal information to a commercial website and learns about the consequences. . . . . . . . 16

Figure 3.2 Screenshots from the remaining 11 scenarios. . . . . . . . . . . 17

Figure 3.3 Demographics of the 16 participants for the JCJF user study. . 19

Figure 3.4 Scoring of answers for the pre-and-post test questions on online privacy 1 = poor, 2 = marginal, and $3=$ excellent. ${ }^{*} \mathrm{P} 5$ 's audio recording was lost. . . . . . . . . . . . . 25

Figure 3.5 Pre-and-post scenario questionnaire responses. Responses were scored and the bar graphs represent the number of participants who answered the right/wrong answer. . . . . . . . . . 26

Figure 3.6 Participants' mean reading times per scenario in seconds. P5's data was not recorded. . . . . . . . . . . . 27

Figure 3.7 Scenarios mean reading times. . . . . . . . . . . . . . . . . . 28

Figure 3.8 Likert scale responses for "The content was Long to Short". . 30

Figure 3.9 Likert scale responses for "The game scenarios were? Boring to Interesting" . . . . . . . . . . . . . . . . . . . . . 30

Figure 3.10 Likert scale response for "Playing the game was? Boring to Exciting". . . . . . . . . . . . . . . 31

Figure 3.11 Likert scale responses for "How much did you learn? Nothing

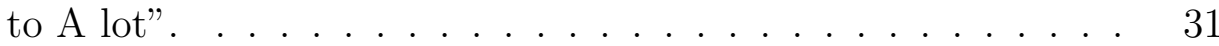

Figure 3.12 Likert scale responses for "The game was? Boring to Fun". . . 31

Figure 3.13 Likert scale response for "The game design was? Ugly to Nice". 31

Figure 3.14 Likert scale response for "Would you recommend this game to a friend? Never to absolutely". . . . . . . . . . . . . . . . . 32

Figure 3.15 Heatmap showing a participant's fixation on Jo's face. . . . . . 33

Figure 3.16 Scan path showing a participant's fixation on Josie's face. . . . 34 
Figure 3.17 Scan path showing a participant's fixation on Josie's face following a zig-zag reading pattern where they read some, look around, and read some, and look around again. . . . . . . . .

Figure 3.18 Scan path showing a participant's fixation on the text alone without referring to Jo's face, following a line-by-line reading pattern . . . . . . . . . . . . . . .

Figure 3.19 Scan path showing a participant's fixation on the forward arrow to move them to the next screen, while ignoring the Jos who were not making eye contact with the camera. . . . . . . . .

Figure 3.20 Scan path showing a participant's fixation of the Cheapmail scenario. Participants were drawn to the title and the coloured button at the bottom of the screen. They spent time exploring the navigation buttons at the top, but skipped the plain text treatment of the body of the email. . . . . . . . . . . .

Figure 3.21 Scan path showing a participant's fixation of the Teenworld.com scenario. Scan path shows how participants spent considerable amount of time trying different link options but were not successful as the Teenworld.com site was not active. The red diamonds represent the participant's clicks. . . . . . . . . . .

Figure 3.22 Scan path showing a participant's fixation on the left hand side tab trying to find out the navigation to the next page. Scan path shows how participants gazed at other links on the left hand side of the screen, while not noticing that only the "schools" link that blinked in red is active. . . . . . . . . . . .

Figure 3.23 Scan path showing a participant's line-by-line and zig-zag reading patterns. Participants some times relied on such mixed reading pattern to take a break from dense text areas. . . . .

Figure 3.24 Scan path showing a participant's skimming over text areas. This screen came as the 4th page of the long Sc 10 (see Figure $3.2 \mathrm{j}-1) \ldots \ldots \ldots \ldots \ldots \ldots$ 
Figure 4.1 The 5 phases of the ADDIE instructional design model. Figure adapted from Wikimedia Commons [11]. . . . . . . . . . 43

Figure 4.2 The main landing page of ADITL. A calendar shows the active days containing scenarios in red. . . . . . . . . . . . . 49

Figure 4.3 The Day View hosts links to scenarios for a particular topic. . 50

Figure 4.4 Scene from the online information verification scenario where Josie sees a post on her social media page with the news that school busses are cancelled and that it is a snow day. . . . . 50

Figure 4.5 First scene of the first scenario in D1 . . . . . . . . . 53

Figure 4.6 Second scene of the first scenario in D1 . . . . . . . 53

Figure 4.7 Third scene of the first scenario in D1 . . . . . . . . . . 54

Figure 4.8 After the player makes a choice, the newsfeed opens to show posts on Josie's social media page. . . . . . . . . . . . 54

Figure 4.9 The end of day summary. The player can scroll to see all of their choices with scores and feedback. . . . . . . . . 55

Figure 4.10 Scene from the photo sharing scenario showing Josie on a field trip and taking picture of her friend Sofia screaming because a butterfly landed on her face. . . . . . . . . . . . . . 56

Figure 4.11 Scene from the online privacy scenario showing Josie looking disheveled at a slumber party. . . . . . . . . . . . 56

Figure 4.12 First Concept's calendar landing page. . . . . . . . . . 57

Figure 4.13 First Concept's day view. . . . . . . . . . . . . . 58

Figure 4.14 Second Concept: The landing page of the second concept, including icons on the active calendar days. . . . . . . . 58

Figure 4.15 Third Concept's calendar landing page. . . . . . . . . . . 59

Figure 4.16 Third Concept's day view. . . . . . . . . . . . . . . . 59

Figure 4.17 Demographics of the eight participants of the pilot user study. 61

Figure 5.1 Demographics of the 14 participants of the ADITL user study. 68 
Figure 5.2 Distribution of scores for each for question 1 from the pre- and post-game day interviews. D1* and D3* are missing the record-

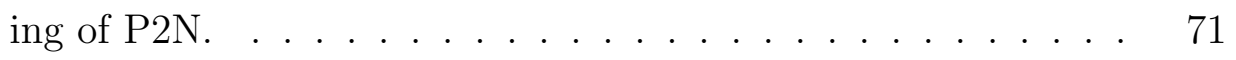

Figure 5.3 Distribution of scores for each for question 2 from the pre- and post-game day interviews. D1* and D3* are missing the record-

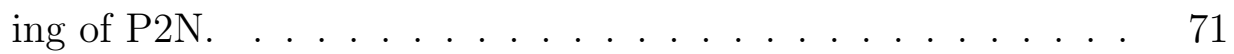

Figure 5.4 Total score earned per day for in-game decisions out of 10 . *D1's maximum score is $9 \ldots \ldots \ldots$. . . . . . . . . 75

Figure 5.5 Number of participants earning each game score for the 5 scenarios of the 5 game days. Three scenarios differed from the norm. D1-Sc3 had a total score of 1 for one choice, and the other two choices were valued at 0. D1-Sc5 and D4-Sc5 had a total score of 2 for one choice, with the other two choices valued at $0 \ldots \ldots \ldots \ldots \ldots \ldots \ldots \ldots \ldots$

Figure 5.6 Reading times per day for each participant. . . . . . . . 77

Figure 5.7 Mean reading times per scenario for each of the game days. . . 78

Figure 5.8 Distribution of Likert scale response for "The content was: Long to Short". . . . . . . . . . . . . . . 80

Figure 5.9 Distribution of Likert scale response for "The game scenarios were: Boring to Interesting" . . . . . . . . . . . . . 8 80

Figure 5.10 Distribution of Likert scale response for "Playing the game was: Boring to Exciting". . . . . . . . . . . . . . . 81

Figure 5.11 Distribution of Likert scale response for "How much did you learn? Nothing to A lot". . . . . . . . . . . . . . . . 81

Figure 5.12 Distribution of Likert scale response for "The game was: Boring to Fun". . . . . . . . . . . . . . . . . . . . 81

Figure 5.13 Distribution of Likert scale response for "The game design was: Ugly to Nice". . . . . . . . . . . . . . . . . . . . . . 81

Figure 5.14 Distribution of Likert scale response for "Would you recommend this game to a friend? Never to Absolutely". . . . . . . . . . 82 
Figure 5.15 Heat map showing a participant's gaze at the game's main and secondary design elements. . . . . . . . . . . . . . . . . 82

Figure 5.16 Heat map showing a participant focusing on the game content. 83

Figure 5.17 Scan path of P7R's gaze towards Josie's phone as it starts to move, indicating that Josie received a text message. . . . . . . 84

Figure 5.18 Portion of heat map showing P14R's gaze on the score and the newsfeed. ...................... 84

Figure 5.19 Scan path shows P5N looking repeatedly at the newsfeed and score (yellow boxes) on the main page. . . . . . . . . . . 86

Figure 5.20 Heat map showing P9N focusing on new social media posts. . 86

Figure 5.21 Scan path showing P10N's line-by-line reading pattern. . . . . 87

Figure 5.22 Scan path showing P13R's zig-zag reading pattern. . . . . . . 87

Figure 5.23 Scan path showing how participants only glanced quickly at the long "fake" textual description. . . . . . . . . . . . . . . 88

Figure 5.24 Likert scale response for "The game design was? Ugly to Nice" for JCJF. . . . . . . . . . . . . . . . . . . . . . . 91

Figure 5.25 Likert scale response for "The game design was: Ugly to Nice" for ADITL. . . . . . . . . . . . . . . . . . . .

Figure A.1 Parental / Legal Guardian Permission for Children Participation in Research page 1. . . . . . . . . . . . . . . . . 115

Figure A.2 Parental / Legal Guardian Permission for Children Participa-

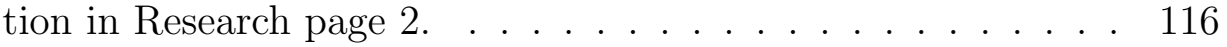

Figure A.3 Verbal Informed Assent Script for the Children . . . . . . . . 117

Figure A.4 Debriefing Form . . . . . . . . . . . . . . . . . . . . . . 119

Figure A.5 User Feedback Questionnaire . . . . . . . . . . . . . . . . . 121

Figure A.6 The main landing page of ADITL. A calendar shows the active days containing scenarios in red. . . . . . . . . . . . 128

Figure A.7 The Day View hosts links to scenarios for a particular topic. . 129

Figure A.8 D1-Cyberbullying: Scene 1 of first scenario. . . . . . . . . . . 129 
Figure A.9 D1-Cyberbullying: Scene 2 of first scenario. . . . . . . . . . . 130

Figure A.10 D1-Cyberbullying: Scene 3 of first scenario. . . . . . . . . . . 130

Figure A.11 D1-Cyberbullying: Scene 1 of second scenario. . . . . . . . . . 131

Figure A.12 D1-Cyberbullying: Scene 2 of second scenario. . . . . . . . . . 131

Figure A.13 D1-Cyberbullying: Scene 3 of second scenario. . . . . . . . . . 132

Figure A.14 D1-Cyberbullying: Scene 1 of third scenario. . . . . . . . . . . 132

Figure A.15 D1-Cyberbullying: Scene 2 of third scenario. . . . . . . . . . . 133

Figure A.16 D1-Cyberbullying: Scene 3 of third scenario. . . . . . . . . . . 133

Figure A.17 D1-Cyberbullying: Scene 1 of fourth scenario. . . . . . . . . . 134

Figure A.18 D1-Cyberbullying: Scene 2 of fourth scenario. . . . . . . . . . 134

Figure A.19 D1-Cyberbullying: Scene 3 of fourth scenario. . . . . . . . . . 135

Figure A.20 D1-Cyberbullying: Scene 1 of fifth scenario. . . . . . . . . . . 135

Figure A.21 D1-Cyberbullying: Scene 2 of fifth scenario. . . . . . . . . . . 136

Figure A.22 D1-Cyberbullying: Scene 3 of fifth scenario. . . . . . . . . . . 136

Figure A.23 The Day View of D2-Data mining topic. . . . . . . . . . . . . 137

Figure A.24 D2-Data mining: Scene 1 of first scenario. . . . . . . . . . . . 137

Figure A.25 D2-Data mining: Scene 2 of first scenario. . . . . . . . . . . . 138

Figure A.26 D2-Data mining: Scene 3 of first scenario. . . . . . . . . . . . 138

Figure A.27 D2-Data mining: Scene 1 of second scenario. . . . . . . . . . . 139

Figure A.28 D2-Data mining: Scene 2 of second scenario. . . . . . . . . . . 139

Figure A.29 D2-Data mining: Scene 3 of second scenario. . . . . . . . . . . 140

Figure A.30 D2-Data mining: Scene 4 of second scenario. . . . . . . . . . . 140

Figure A.31 D2-Data mining: Scene 1 of third scenario. . . . . . . . . . . . 141

Figure A.32 D2-Data mining: Scene 2 of third scenario. . . . . . . . . . . . 141

Figure A.33 D2-Data mining: Scene 3 of third scenario. . . . . . . . . . . . 142

Figure A.34 D2-Data mining: Scene 1 of fourth scenario. . . . . . . . . . . 142

Figure A.35 D2-Data mining: Scene 2 of fourth scenario. . . . . . . . . . . 143

Figure A.36 D2-Data mining: Scene 3 of fourth scenario. . . . . . . . . . . 143

Figure A.37 D2-Data mining: Scene 1 of fifth scenario. . . . . . . . . . . . 144

Figure A.38 D2-Data mining: Scene 2 of fifth scenario. . . . . . . . . . . . 144 
Figure A.39 D2-Data mining: Scene 3 of fifth scenario. . . . . . . . . . 145

Figure A.40 The Day View of D3-Internet privacy topic. . . . . . . . . . 146

Figure A.41 D3-Internet privacy: Scene 1 of first scenario. . . . . . . . . 146

Figure A.42 D3-Internet privacy: Scene 2 of first scenario. . . . . . . . . 147

Figure A.43 D3-Internet privacy: Scene 3 of first scenario. . . . . . . . . . 147

Figure A.44 D3-Internet privacy: Scene 1 of second scenario. . . . . . . . . 148

Figure A.45 D3-Internet privacy: Scene 2 of second scenario. . . . . . . . . 148

Figure A.46 D3-Internet privacy: Scene 3 of second scenario. . . . . . . . . 149

Figure A.47 D3-Internet privacy: Scene 1 of third scenario. . . . . . . . . 149

Figure A.48 D3-Internet privacy: Scene 2 of third scenario. . . . . . . . . 150

Figure A.49 D3-Internet privacy: Scene 3 of third scenario. . . . . . . . . . 150

Figure A.50 D3-Internet privacy: Scene 1 of fourth scenario. . . . . . . . . 151

Figure A.51 D3-Internet privacy: Scene 2 of fourth scenario. . . . . . . . . 151

Figure A.52 D3-Internet privacy: Scene 3 of fourth scenario. . . . . . . . . 152

Figure A.53 D3-Internet privacy: Scene 1 of fifth scenario. . . . . . . . . 152

Figure A.54 D3-Internet privacy: Scene 2 of fifth scenario. . . . . . . . . 153

Figure A.55 D3-Internet privacy: Scene 3 of fifth scenario. . . . . . . . . 153

Figure A.56 The Day View of D4-Image sharing topic. . . . . . . . . . 154

Figure A.57 D4-Image sharing: Scene 1 of first scenario. . . . . . . . . . . 154

Figure A.58 D4-Image sharing: Scene 2 of first scenario. . . . . . . . . . 155

Figure A.59 D4-Image sharing: Scene 3 of first scenario. . . . . . . . . . 155

Figure A.60 D4-Image sharing: Scene 1 of second scenario. . . . . . . . . 156

Figure A.61 D4-Image sharing: Scene 2 of second scenario. . . . . . . . . 156

Figure A.62 D4-Image sharing: Scene 3 of second scenario. . . . . . . . . 157

Figure A.63 D4-Image sharing: Scene 1 of third scenario. . . . . . . . . 157

Figure A.64 D4-Image sharing: Scene 2 of third scenario. . . . . . . . . 158

Figure A.65 D4-Image sharing: Scene 3 of third scenario. . . . . . . . . 158

Figure A.66 D4-Image sharing: Scene 1 of fourth scenario. . . . . . . . . 159

Figure A.67 D4-Image sharing: Scene 2 of fourth scenario. . . . . . . . . 159

Figure A.68 D4-Image sharing: Scene 3 of fourth scenario. . . . . . . . . 160 
Figure A.69 D4-Image sharing: Scene 1 of fifth scenario. . . . . . . . . . 160

Figure A.70 D4-Image sharing: Scene 2 of fifth scenario. . . . . . . . . . 161

Figure A.71 D4-Image sharing: Scene 3 of fifth scenario. . . . . . . . . . 161

Figure A.72 The Day View of D5-Verification of information from online sources topic. . . . . . . . . . . . . . . . . 162

Figure A.73 D5-Verification of information from online sources: Scene 1 of first scenario.

Figure A.74 D5-Verification of information from online sources: Scene 2 of first scenario.

Figure A.75 D5-Verification of information from online sources: Scene 3 of first scenario.

Figure A.76 D5-Verification of information from online sources: Scene 1 of second scenario. . . . . . . . . . . . . . . . . . 166

Figure A.77 D5-Verification of information from online sources: Scene 2 of second scenario.

Figure A.78 D5-Verification of information from online sources: Scene 3 of second scenario. . . . . . . . . . . . . . . . . . . 168

Figure A.79 D5-Verification of information from online sources: Scene 1 of third scenario. . . . . . . . . . . . . . . . . . . . 169

Figure A.80 D5-Verification of information from online sources: Scene 2 of third scenario. . . . . . . . . . . . . . . . . . 170

Figure A.81 D5-Verification of information from online sources: Scene 3 of third scenario. . . . . . . . . . . . . . . . . . . . . . . . 171

Figure A.82 D5-Verification of information from online sources: Scene 1 of fourth scenario. . . . . . . . . . . . . . . . . . 172

Figure A.83 D5-Verification of information from online sources: Scene 2 of fourth scenario. . . . . . . . . . . . . . . . . . 173

Figure A.84 D5-Verification of information from online sources: Scene 3 of fourth scenario. . . . . . . . . . . . . . . . . . . . . . . 174 
Figure A.85 D5-Verification of information from online sources: Scene 1 of fifth scenario. . . . . . . . . . . . . . . . . 175

Figure A.86 D5-Verification of information from online sources: Scene 2 of fifth scenario. . . . . . . . . . . . . . 176

Figure A.87 D5-Verification of information from online sources: Scene 3 of fifth scenario. . . . . . . . . . . . . . . . 177 


\section{Chapter 1}

\section{Introduction}

\subsection{Motivation}

As of 2015 there were 3,366 million Internet users [31] and this number continues to increase. According to a 2013 Pew research study, 74\% of the teens 12 to 17 access the Internet on cell phones, tablets, and other mobile devices at least occasionally [39]. Additionally, in 2010, 98\% of Canadian children in grades 4-11 had access to the Internet outside of school [80]. A 2007 study of 40,000 New York students [42] found that $48 \%$ of children as young as 5-6 years are online regularly and only half of those students reported that their parents watch them as they use the computer.

The need to educate children on ways to protect their online privacy and security is pressing, as more and more children are going online unsupervised. Steeves' report [79] to the Office of the Privacy Commissioner of Canada emphasizes the need for educational initiatives. Sharples et al. [76] similarly advocates for empowering children by teaching secure and responsible web behaviour while promoting a safe environment.

Children need to understand in a relevant manner the negative effects of not protecting their online security and privacy. However, convincing them of its importance is a challenge, as users in general do not think of protecting their online privacy and security as their primary task [85].

In Canada, the main source of educational games on this subject is MediaSmarts [49]. MediaSmarts is a not-for profit organization based in Ottawa but with national reach. Their main goal is to promote digital literacy in children and teach them to be good digital citizens. They have developed online educational games and tutorials that can be used within the classroom by teachers to educate children of different ages. However, given how quickly technology evolves, many of their educational tool are now outdated. We have partnered with MediaSmarts through 
MITACS to create new interactive educational material and to empirically evaluate the effectiveness of these approaches.

\subsection{Research Challenge}

The challenge addressed in this thesis is to design an educational online game to teach children aged 11 and 12 different methods to protect their online privacy and security. Specifically, our research questions are as follows:

1. How do we make education of privacy and security relevant to children's everyday life?

2. Do integrated interactive educational games form an effective, and enjoyable method for learning complex concepts like online privacy and security to children?

\subsection{Contributions}

The main contributions of this thesis are:

1. The design and prototyping of an online educational game that teaches children (11 - 12 year olds) ways to protect their online privacy and security.

2. Empirical evaluation showing improved effectiveness of the new game over an existing game, including eye-tracking data showing improved interaction with the new game elements.

1. The design and prototyping of an online educational game that teaches children (11 - 12 year olds) ways to protect their online privacy and security: Using a user-centred and educational design approach, we iteratively designed a new game entitled "A Day in the Life of The Jos" (ADITL). In collaboration with MediaSmarts, we iteratively designed an online game to be deployed in a classroom setting and to be included as part of the online privacy and security pedagogical curriculum. I designed the conceptual framework for the game, the game mechanics, the user interface, and the visual elements of the game. This 
included writing the initial scenario scripts, designing the overall look-and-feel of all the game interactions, illustrating the multiple screens for each of the 25 scenarios, and defining the game elements. Work on the game elements included designing the visual interface and interaction of the newsfeed and the scoring mechanism to report the consequences of users' choices.

2. Empirical evaluation showing improved effectiveness of the game over an existing game, including eye-tracking data showing improved interaction with the new game elements: We incorporated children's feedback and opinion through the multiple steps of our design and development process. We first started by performing user study of the existing game, Jo Cool or Jo Fool (JCJF). Based on the children's feedback and eye-tracking results, we decided on elements of the existing game to incorporate in the new game's design and which features to improve. As part of the design process, we conducted a pilot user study, where we tested 3 different visual design prototypes and the proposed storyline with children. Finally we conducted a third user study evaluating a medium fidelity prototype of ADITL, showing improved engagement with the learning material. Additionally, we compared the learning outcomes and the children's preferences of ADITL over JCJF.

\subsection{Thesis Outline}

The following are the different sections of this thesis: Chapter 2 reviews the background research in online privacy education, prior games developed to teach both adults and children about different security and privacy issues, and different educational methods intended for children. In Chapter 3, we summarize our user study of the old game (JCJF), highlighting main findings. Chapter 4 details our instructional design process, the design of the new game: "A Day in the Life of the Jos" (ADITL), and the ADITL second prototype. Chapter 5 describes the user study evaluating the new ADITL game, and compares results of the two games. We conclude with Chapter 6 , where we discuss our findings, limitations, and future work. 


\section{Chapter 2}

\section{Background}

In this chapter, we give background on areas relevant to the thesis. We specifically focus on literature in children's online privacy and security education with regards to games developed for that purpose. We shed light on children's developmental characteristics. We highlight children's use of mobile devices and social media. We discuss technology use within the classroom, particularly the use of games in formal education. Lastly, we give general game design strategies applicable to our work.

\subsection{Children's Developmental Characteristics}

After examining HCI, educational, and psychological literature on child development, we discovered that they mainly fell within these two areas: cognitive and physical development, and social and emotional development [8] [40] [64].

\subsubsection{Cognitive and physical development}

Cognitive Development: Cognitive development is the child's ability to process information, think perceptually and master a particular language [40]. It is the mental and intellectual growth of a child [8]. Children aged 11 to 12 years old have developed the ability of logical thinking, deductive reasoning, and complex problem solving on a smaller scale [23].

Mental Development: Eleven and twelve year olds have mentally developed to consider an idea-based thought pattern [65]. Their brains have rapidly developed, enabling them to consider various solutions without having to act them out. They can also deal with hypothetical situations [65]. They can think in an abstract manner and imagine different potential outcomes for a situation [23].

Literacy: Children aged 11 and 12 years have developed strong reading skills, as well as communication skills. Their focus is to express themselves so they aim 
to harness their vocabulary and mastery of the language, with proper spelling and writing [19].

Specialization: 11 to 12 year olds have the ability to become an expert in one particular area, and they perceive themselves as specialists, not generalists [23].

Physical Development: Children of this age group tend to have gained much control over their fine and gross motor skills. They are comfortable using digital devices and manipulating a mouse as an input device [8].

\subsubsection{Social and emotional development}

Socialization: During this stage, children develop strong ties with their peers; adult influence decreases in comparison with their peers' influence [19]. Same-gender best friends start to develop as well. This age is also the start of puberty, where emotions quickly changes from one extreme to another [19]. Children at this stage like to socialize; however, they find themselves increasingly relying on electronic socializing with the ubiquitous use of mobile devices. A study performed by Kaplan et al. [34] showed how children (11-14 years) preferred communicating via digital devices even within the same room while performing other tasks like reading on a digital library. They switched between the reading app and the instant messenger app to communicate with their peers digitally.

Emotional development: Children of this age start to develop a sense of individuality [23]. Decision making becomes harder for them as they are able to see other sides of the equation [19]. Their creative thinking enables them to develop their own scenarios and think of possible consequences [21] [20]. According to Fisher in her book "Designing Games for Children" [19], children aged 11 - 12 years stop watching children's programs and start watching adult shows. This lack of interest in younger programs reflects their aspiration to be considered as adults [23].

Motivation and Engagement: Children's motivation to use technology is inherently high, however, if the app, or medium they interact with is not exciting they lose interest quickly [8]. Research done on children's engagement in multimedia found that children (9-14yrs) want to maintain control over the environment and affect the outcomes of different situations [74]. This also allows children to make choices and 
learn from their own mistakes by understanding the consequences of their actions.

\subsection{Children and Media}

\subsubsection{Use of social media}

Interest in Social Media apps: As 11 and 12 year olds are figuring out their special interests, they want to share those interests with other like-minded children of their age. They regularly use apps such as Instagram [30] and sites like Tumblr to publish their thoughts and hobbies [82]. According to Rainie [68], approximately a quarter of teens use Instagram, 1 in 7 use Pinterest, and 1 in 10 uses Tumblr. They are aware of popular social media sites like Facebook and Twitter; they probably watch their parents' use of them regularly, however, most do not have their own accounts yet. This might be due to the age restrictions that some parents might have placed due to fear of malicious strangers, since according to Pew research, one in three parents are concerned about technology use in the last year (2015) [16]. Another reason where children have limited access to social media accounts is because of the age restrictions on the sites themselves. Facebook, for example, has a minimum age restriction of 13 years old [18] in order to create an account.

\subsubsection{Use of mobile devices}

According to Pew research, 74\% of the teens in the U.S. 12 to 17 access the Internet on cell phones, tablets, and other mobile devices at least occasionally as of 2013 [39], 78\% of teens now have a cell phone, and almost half (47\%) of those own smartphones [39]. That translates into $37 \%$ of all teens having smartphones, up from just $23 \%$ in 2011. Children of this age group rely on mobile devices more than their computers. They have stopped using it solely for entertainment and started to use it more as a tool to obtain information [23].

\subsection{Technology in the Classroom}

Since the early 90's, use of technology in the classroom has been increasing [67]. By 2009, $97 \%$ of classrooms in the US had one or more computers [67], 93\% of those 
computers had access to the Internet, and every five students shared a computer [67]. In recent years, many schools allow children to bring their own devices [75], and or provide them with mobile devices owned by the school [33]. The following is a review of some of the technology used within the classroom.

The use of Smart Boards within the classroom for interactive teaching and learning is a popular use of technology [33]. Google Classroom [24] is another recent example of use of technology within the classroom. Teachers can use Google tools to share material with students and allow collaboration between students on school projects. By working on a common Google shared drive, the students are able to work coherently on a shared project in a one document at the same time [25]. The above are two examples of the use of commercial technology within the classroom.

In the research domain, Vihavainen et al. [84] proposed the use of a hybrid approach in children's primary education. Through the use of both mobile smart phones and printed books; primary school age students can access extra curricular activities on their mobile phones. Using the IMediaLink application on smartphones that were given to the students for the course of the user study. The students would capture a screenshot of the printed textbook page they are studying. The system identifies the textbook page, and draws the accompanying exercise. If the students accomplished the tasks correctly, the system alerts the teacher automatically. The teacher used SMS to communicate with the students outside the classroom. Although the results of the one-week study were positive, with 21 of the 22 students in favour of the new approach, more research is needed to evaluate this hybrid approach for a longer duration of time.

\subsection{Educational Games}

The use of games in education is an effective method for learning [35]. Playing the game becomes the primary focus of the user and the learning happens on an almost subconscious level [9]. Children of this age group are unlikely to pick up an educational game for the sake of learning about a new topic. Unless the game was assigned by the school teacher, the possibility of them choosing to play an educational game is low [19]. That is why it is important that apps targeted towards children's education 
include inherent motivators to allow children to spend time and absorb the intended learning [8].

Games with an educational intention can have different goals than games created for children to play just for fun. Games that teach math or other school subjects have a clear goal that is to educate children in a specific field. However, some games have an inherent educational nature, even though its main purpose was for fun. A good example of this type of games is Minecraft [56],which is very popular among 11 and 12 year olds [57]. Through game play, the children learn about social interactions as they interact with other players online. Additionally, they learn about engineering as they construct the different buildings of their fortresses. A second example is Kingdom Rush [32], which relies on the use of storyline and characters. The game involves strategy, design, fantasy, and financial management, where the players have to decide on the type of tower to build to secure their kingdom based on the budget they have and the type of attacking enemy.

\subsubsection{Games in the classroom}

There are many games that has been introduced in the classroom, we discuss three examples for this age group.

Through two workshops, Brigas et al. [7] involved elementary students in the development of their Simulkids tool. Simulkids is an online modelling and simulation tool developed to help elementary students understand complex cause and effect georeferenced information, through visual and auditory feedback. The researchers tested the Simulkids prototype and asked for the students' feedback in developing other georeferenced scientific concepts.

The work of Danesh et al. [12] builds upon children's ability (11 - 13 years) to learn through metaphors, by creating Geney, a collaborative game that allows the children to learn about genetics with the use of mobile devices. The children each have their own fish on their device and must collaborate with other children to mate fish and produce offsprings with specific characteristics. Children were able to grasp the metaphor of a pond successfully, could explore how characteristics combine, and understand the genetics concepts. 
Stories can become a great tool for learning and can impact change [72]. Rubegni and Landoni designed "Fiabot!", a Digital StoryTelling (DST) iPad application that helps grades 4 and 5 students (aged 9 - 11) in their story creations. This aspect of allowing children to create their own stories is important because it feeds into their creativity. It also allows them to experiment with the idea of how certain actions lead to certain consequences. "Fiabot!" has been successfully implemented within a classroom setting.

\subsection{Security Education}

We now focus on security education since this is the topic of the thesis. We will address the following issues: general security education, the use of educational comics, and games that teach about security.

Users are normally interested in accomplishing a task online, and security is not their primary goal [85] even though it is important. Large Internet companies and financial institutions have published extensive online help to guide users on how to protect their online privacy and security. Companies like Ebay [17], Microsoft [55], Google [26], Paypal [63] offer tips and tools to educate users about security. The advice they offer focuses on a list of what users should, and should not do. However, the use of text based recommendations-only advice is ineffective [88]. Low motivation and poor understanding of online security threats drive users to noncompliance with security guidelines [1]. Both adults and young users need explanations as to why these guides and regulations are important and how their actions can diminish vulnerability to threats. They need to understand in an applied manner the potential negative consequences that result from ignoring these security guidelines.

In a corporate environment, some companies have deployed embedded training tools. One example is "PhishGuru" [37], an email-based training system where users are sent fake phishing emails by the system administrator or from a training company and must recognize those among their regular email. Although it is not a game, users are learning while undertaking everyday email activity. So, the learning is happening in an indirect manner, and the user is exposed to phishing without the real threat of an actual attack. This allows the user to practice the required level of vigilance. 
When tested in real life setting, PhishGuru proved to be a successful way to teach users about ways to identify phishing [36].

\subsubsection{Comics to teach security}

Adults: The use of cartoons and comics has also proven to be an effective method for learning [59]. Comics help present serious topics like cybersecurity in a light non-intimidating manner. Security Cartoons [78] are a set of short comic strips that specializes in educating a general audience about different security topics. Popular comics such as Dilbert [2], and XKCD [58] also sometimes discuss security issues. Google Chrome's use of comics to describe its security features is also a commendable effort [41]. The 39-page instruction manual is intended to interest the reader in cybersecurity and illustrate how can they protect themselves while using Chrome. Zhang-Kennedy, Chiasson, and Biddle [89] designed online comics using metaphors to teach users about viruses and malware, password guessing attacks, and how to protect their privacy online, which proved to be an effective in teaching users how to protect themselves. The results of their user study showed improvement in participants' retention of the learned knowledge as well as their behaviour in the following days.

Children: Social Smarts [60] is a graphic novel created by the Office of the Privacy Commissioner of Canada to help young Canadians better understand and navigate privacy issues in the online world. The 12-page graphic novel targets tweens and younger teens. Another example targeted for a younger audience (children aged 7 to 9) is Cyberheroes [87], an interactive e-book that helps children understand about online privacy risks. Results of their initial user study shows that both parents and children thought it was a fun and engaging educational tool.

\subsubsection{Security games}

Adults: An early game in security education is Anti-Phishing Phil [77]. The game's interactive approach aims at teaching players ways to recognize suspicious URLs. The game was effective in teaching players to identify fraudulent web sites compared to a control group.

The Auction Hero [9] computer game is another example of enabling the user to 
learn security concepts while playing a game. The player is in training to become an "auction hero" by assembling robots and selling them on a fictitious online market, while minding the antivirus protection and warding off phishing emails. While managing robot production and sales, the player must be vigilant for malware protection updates and spoofed emails. The player is monitoring security as a secondary goal, just as in real life, and this knowledge can be transferred to real life situations.

Control-Alt-Hack [13] is a tabletop recreational card game that teaches security concepts to computer science students and developers. The game goal is to use everyday events and turn them into a cybersecurity challenge, where the players have to learn new information in order to solve the mission. According to Denning et al., the creators of Control-Alt-Hack, the use of games allow for exploration and asking questions [13]. The players are in a fun and relaxed state of mind, which allows them to engage better with the game's situations.

Children: Co-Co's AdverSmarts [45] is an interactive online game designed for children 5 to 8 years old to recognize the marketing techniques of commercial websites that target children, in order to build their brand loyalty and improve popularity. Within the game, children create a website through selecting special features. CoCo, the main character, explains how marketers use these features to improve the popularity of the site.

Privacy Pirates [50] is another interactive online game-based tutorial that introduces children, ages 7 to 9 , to the concept of online privacy. The game teaches ways to distinguish between appropriate personal information to give out and others to keep private. The children assemble a map that leads to a pirate's treasure. While trying to answer privacy and personal information questions on the Internet, children collect pieces of the map when they make correct choices.

Privacy Playground [51] and Cybersense and Nonsense [46] teach children ages 8 to 10 year olds on ways to decipher marketing ploys online, how to verify online information and how to observe proper behaviour when dealing with others online.

Gaming Privacy [69] is a hybrid game targeting children aged 10 to 12 , where the players interact with an online video-game along with a physical board-game. The game was designed in collaboration with 7 children to establish the game's privacy 
goals and the game mechanics. Within the fictitious setting of "Atlantic" city, players must determine with which game individuals or companies they should share their private information. The game also teaches consequential thinking of the different choices made, as well as autonomous privacy decision-making skills.

Click if You Agree [44] is another game to teach children between the ages of 12 to 14 to develop skills and confidence in how to read privacy policies and terms of use instead of blindly accepting them by clicking the "I agree" button. The player selects three privacy related keywords for their scanner. The player navigates among different paragraphs of the privacy policy text. Once the scanner detects a keyword, it beeps and flashes. The player must then click on the paragraph that they think discusses this topic.

\subsection{Design Strategies for Games}

\subsubsection{General strategies}

Feedback: Children of this age-group anticipate immediate and frequent feedback for their actions. They need the assurance that they are performing the right task, especially when learning new skills [4].

Control: One way to keep children's attention is to develop unconventional designs that better suit their taste and imagination. Allow for the children to be in control of the in-game actions. They should have the ability to affect the game and guide the sequence of events [15] [29].

Communication: At this age, a game can include simple visual menus and help functions and expect the young users to use them [8]. Children of this age often feel comfortable using apps geared towards adults [23]. However, designers have to still be cognizant of their limited vocabulary. The language used in the interface still needs to be simpler than for adults [19]. Additionally, children of this age-group prefer visual designs more than ones that heavily rely on textual presentations [19], with the use of metaphors for complex concepts [23]. 


\subsubsection{Learning strategies}

On-screen characters: The presence of onscreen characters also acts as a motivator for children to achieve the intended learning within a system. Lester et al. [38] investigated five different versions of Herman the Bug, from extremely animated with voice overs to muted type. They found that just the presence of a character onscreen made the educational game more fun and that students liked to come back to the game because of its presence. However, caution is needed in regards to the timeliness and the type of feedback or comments such onscreen characters give, as this can have an aversive effect [29].

Extrinsic rewards: The use of extrinsic rewards is another motivator, especially in developing educational games for children [29]. The presence of a scoring system, feedback, or bonus activities allows the children to engage more fully with the learning portion of the educational game. Deci and Ryan [73] point out the importance of having intrinsic and extrinsic rewards working together to strengthen the players' autonomy. They state that the players' autonomy is the main driving factor that will have the greater benefit on the longer run. Peters, in his book Interface Design for Learning [64], also explains that extrinsic motivation is important when intrinsic motivation is lacking.

\subsection{Summary}

With the rise of children's use of mobile devices and Internet access, there is a clear need to educate the children on ways to protect their online privacy and security. We surveyed the literature for alternative ways of educating children. We found that the use of games in education can successfully teach children in an indirect way and focus our efforts in this direction.

In the next chapter, we describe the user testing of an online security and privacy game, then identify areas of improvement to better relate to children's every day life and ensure a better learning experience. 


\section{Chapter 3}

\section{Initial User Study of Existing Game "Jo Cool or Jo Fool"}

"Jo Cool or Jo Fool" [47] is an interactive online educational game presented as a Cyber Tour of twelve mock websites. The game's goal is to educate children ages 11 to 13 years (grades 6 to 8 ) about different cyber security and online privacy topics, including online marketing, e-commerce and data collection, online safety, authenticating online information, and responsible Internet use. It was first released by MediaSmarts in 2000, and is still used in classrooms across Canada. Players explore a given webpage and must decide whether it is trustworthy. Players must decide whether the main characters, siblings Jo and Josie, have made wise decisions with respect to the websites. By examining each website's behaviour, the player learns about some online cyber security risks and how to deal with them. The game can be accessed via the MediaSmarts ${ }^{1}$ website, with an accompanying section specifically for teachers, and another for kids.

We conducted an eye-tracking user study to discover areas of weaknesses and strengths in "Jo Cool or Jo Fool" (JCJF) [47]. This is the first step towards a redesign of the overall look-and-feel and a content update. The JCJF user study included semi-structured interviews to better understand children's usage of the Internet and their basic understanding of online privacy and security. The interview questions additionally explored the children's opinions of the game. In this chapter, we present the study and the results of qualitative and quantitative data analysis.

\subsection{Details of JCJF}

The game features two main characters, a brother and a sister, Jo and Josie, who encounter different "tricky" situation while surfing the web. The players decide if the Jos made the "cool" or "fool" decision. The game suggests that the players use a

\footnotetext{
${ }^{1}$ http://www.mediasmarts.com
} 
checklist of questions to help determine the validity and the safety of each website before evaluating the Jos' choice. The checklist includes the following questions:

- What kind of website is this?

- What decision does Jo/Josie have to make?

- What should Jo/Josie be looking out for?

- Does Jo/Josie make the right decision?

- Why or why not?

The JCJF game is a web-based Flash game operating in a fixed 640 x 480 pixel window. The main colours of the game are red and orange, with white and black text, along with splashes of red and orange text. The game has animated elements such as flashing text or flashing buttons indicating where to click on the page. JCJF is composed of 12 scenarios (Sc 1 through Sc 12), where each scenario take the Jos through a tricky situation and asks the player to help the Jos make the right decision. The scenarios are text heavy, where the player has to read through each slide before clicking on a link that gets them to the next page of text. The game choices are in multiple choice format. Figure 3.1 shows the JCJF's landing page, and one full game scenario. Figure 3.2 shows thumbnails of the remaining 12 scenarios.

According to the teacher's guide [48], the 12 scenarios can be classified into four main topics. For a detailed description for each of the scenarios, see Appendix A.1.

1. Online Marketing, E-Commerce and Data Collection

- Sc 1: Splurge Cola.

- Sc 2: CDRama.

- Sc 6: Lotsa Music.

2. Online Safety

- Sc 3: Fast Talk.

- Sc 10: My Home Page. 

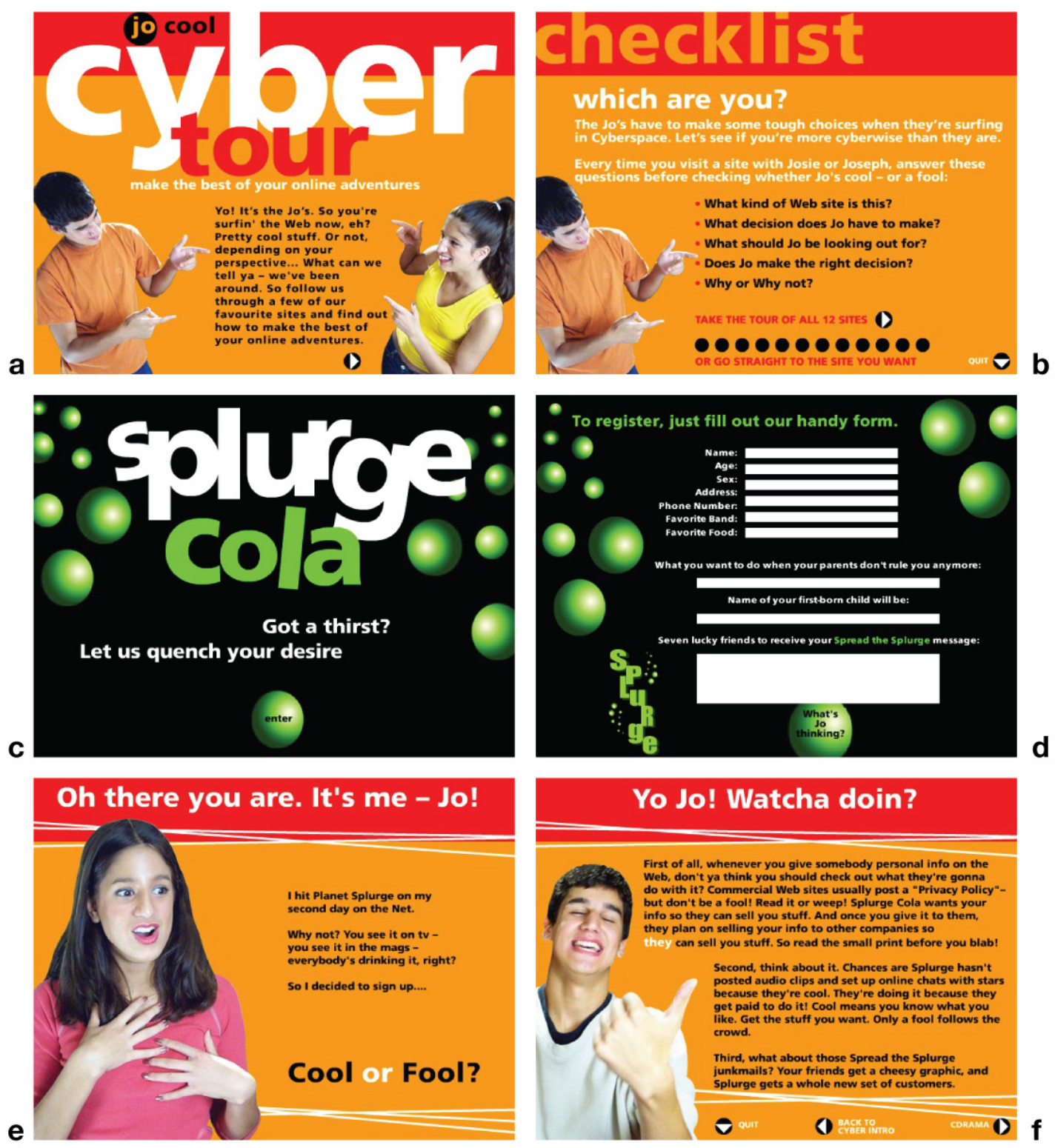

Figure 3.1: (a) JCJF landing page. (b) Main navigation page. (c) through (f) show the sequence of screens of Sc1: Splurge Cola where Josie chooses to provide her personal information to a commercial website and learns about the consequences. 


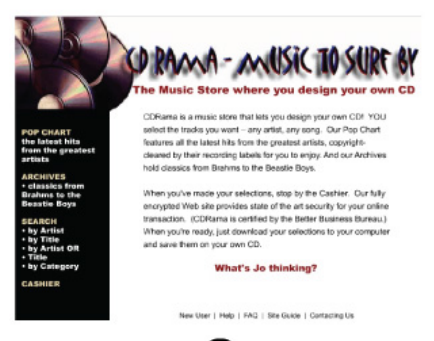

a
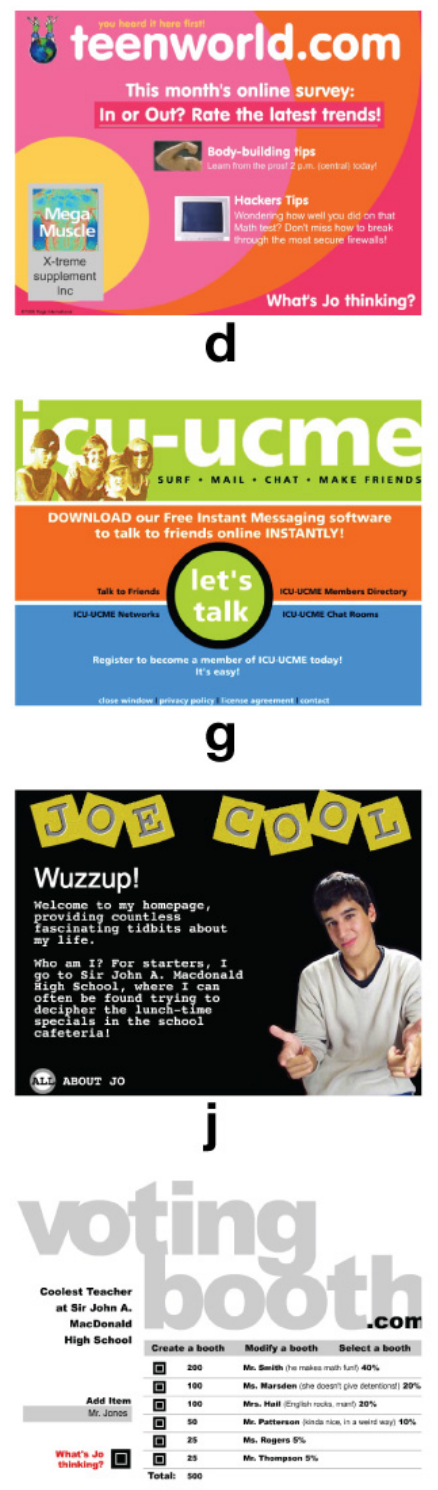

m

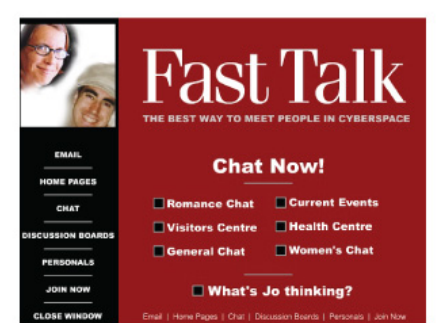

b
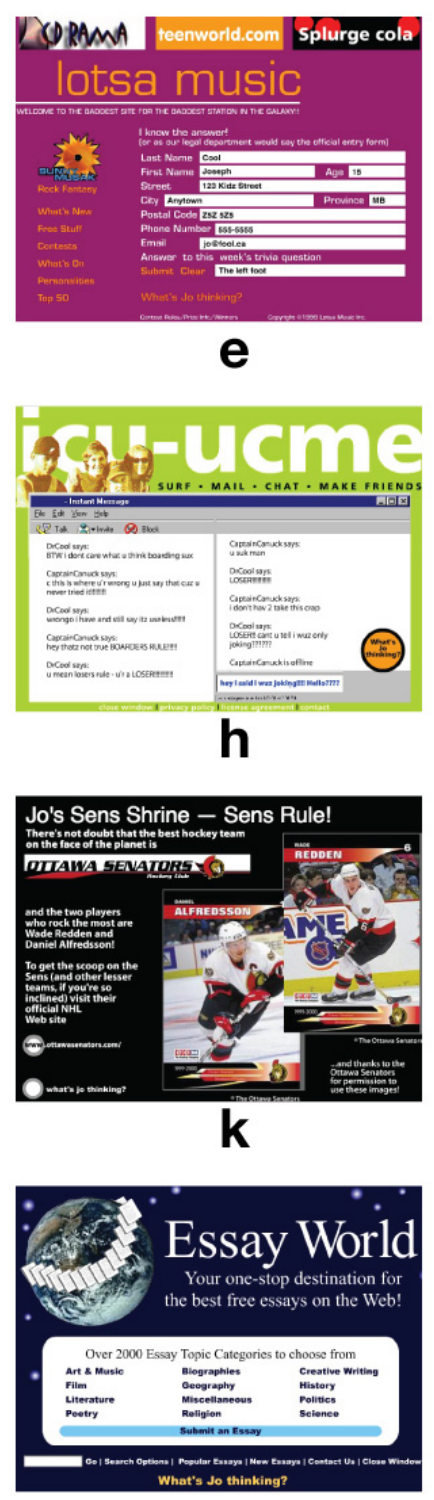

n
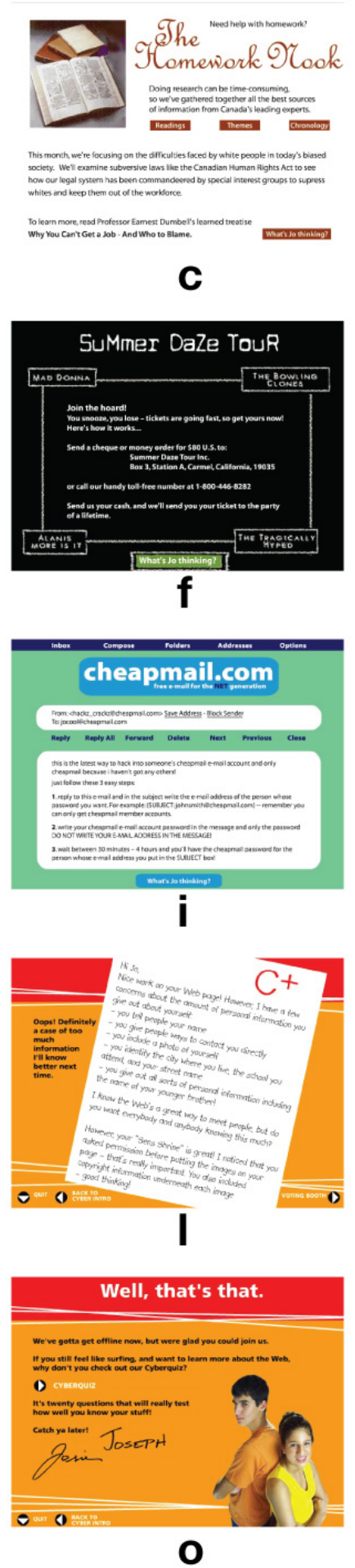

Figure 3.2: Screenshots from the remaining 11 scenarios. 
3. Authenticating Online Information

- Sc 4: Homework Nook.

- Sc 5: Teen World.

- Sc 9: Cheap Mail.

4. Responsible Internet Use

- Sc 8: ICU-UCME.

- Sc 11: Voting Booth.

- Sc 12: Essay World.

\subsection{Research Topics}

We investigated three main aspects of JCJF: its content length, the relevance of its topics, and the overall appeal of its look-and-feel. For the content length, we explored whether players were comfortable with the description of the scenarios, choices, and solutions and whether they were entertained enough to read through all of the text. Since the game was designed and published in the early 2000s, we explored which scenarios were still relevant to players' day-to-day life, and which no longer applied. Finally, we explored the game's visuals and overall design. We identified what the players liked of the game's design and what could be improved.

\subsection{Methodology}

\subsubsection{Ethics}

We submitted a Minimal Risk Ethics Protocol Application in the Spring of 2015. The Carleton University Research Ethics Board determined that the project met appropriate ethical standards as outlined in their Tri-Council Policy Statement: Ethical Conduct for Research Involving Human, 2nd edition, and the Carleton University Policies and Procedures for the Ethical Conduct of Research. Ethics clearance was given on 03 June 2015 until 31 May 2016. 

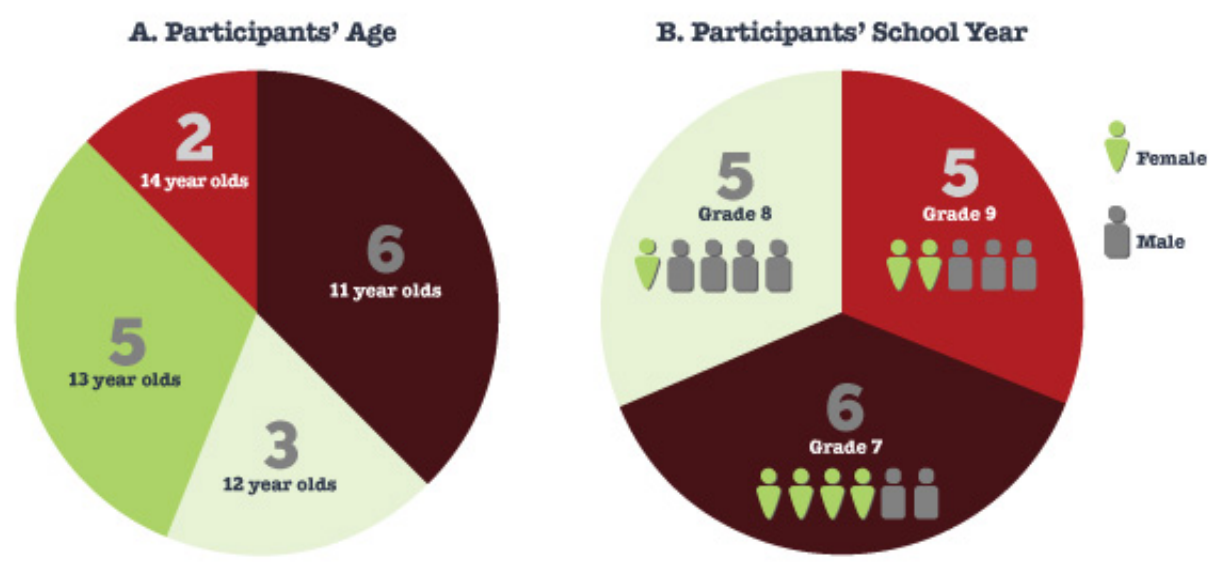

Figure 3.3: Demographics of the 16 participants for the JCJF user study.

\subsubsection{Recruitment and Sampling}

Participants were primarily recruited locally via Facebook parent and community group pages. A public announcement of the user study was posted and participants contacted the researcher via Facebook direct messaging. Siblings from the same household that fit the age range were welcomed to participate in the study, each child participant in their own session, apart from their sibling. Sixteen children aged 11 to 14 years (grades 7 to 9 ) tested JCJF. There were 7 girls and 9 boys. Figure 3.3 summarizes the participants' ages and school grades. The children reported the grade they will be attending the following school year as the study ran during the summer of 2015. One participant's audio and eye tracking recordings were lost due to a technical error. We include data of this particular participant where available.

\subsubsection{Procedure}

Each session lasted 45 minutes on average and was conducted according to the following steps:

1. Consent form: Participants were greeted and parents completed the parental permission form. Verbal informed assent was obtained from the child.

2. Eye tracker: We used the SMI RED 250 MOBILE eye tracker with one-point 
calibration, along with the SMI Smart Recorder software to record the participants' on-screen eye gaze. A webcam with its lens covered recorded the synched audio.

3. Pre-test interviews: The participants were asked questions in a semi-structured interview. The purpose was to investigate the children's basic device use, online and offline. Additionally, we explored their understanding of online privacy and security topics, and how they protect themselves.

4. Pre-scenario questionnaire: Participants completed from two to five multiple choice questions relating to the scenario. When possible, we used the existing questions from the game. We devised some supplementary questions for scenarios where the game questions were repetitive or outdated.

5. Scenario viewing: Participants read through the scenario and were encouraged to think out loud. They read through the whole scenario before moving to the post-scenario questions.

6. Post-scenario questionnaire: Participants repeated similar multiple choice questions to gauge if they learned any new information from the scenario. The researcher also probed into what they valued most and least in the scenario they just viewed.

7. Post-test interview: The researcher repeated questions relating to understanding of online privacy and security and ways to protect themselves. This was done to gauge whether participants gained new insight while playing JCJF. Steps 4 to 7 were repeated for 2 to 3 scenarios as time allowed.

8. User feedback questionnaire: At the end of the session, the participants completed a user feedback questionnaire composed of 5-point Likert scales rating their perception 1) content length, 2) game scenarios, 3) game play, 4) learning, 5) game fun, 6) game design, and 7) game recommendation.

9. Conclusion: Each participant received a $\$ 20$ gift certificate as a compensation. The parent received the Debriefing form and signed a receipt of payment. 
The first 4 participants tested 3 scenarios each. However, we discovered that this took a long time. We had the subsequent participants test with only 2 scenarios. Table 3.1 shows the number of times each scenario was tested. The order of the scenarios was randomly assigned to participants.

\begin{tabular}{|l|r|}
\hline Scenarios & No. of Views \\
\hline S1 & 5 \\
S2 & $* 2$ \\
S3 & 2 \\
S4 & $* 2$ \\
S5 & 5 \\
S6 & 2 \\
S7 & 2 \\
S8 & 3 \\
S9 & 3 \\
S10 & 3 \\
S11 & 5 \\
S12 & 2 \\
\hline \hline
\end{tabular}

Table 3.1: Number of views for each scenario. ${ }^{*}$ For S2 and S4, we lost the audio and eye-tracking video recording of one participant due to a technical error, leaving 2 full data sets and one partial data set for these two scenarios.

\subsection{Results}

We analyze both qualitative and quantitative data collected, including relevant findings of the SMI's eye tracking software, to determine the areas of weakness and strength in JCJF. The data includes participants' gaze fixations on the different components of the game, the time to read its contents, and user feedback from the interviews and questionnaires.

\subsubsection{Device Usage and Context}

We asked participants about the different ways they get online, and their favourite sites and applications online. Additionally, we asked about passwords for different accounts and whether they share them with family members or friends. We also explored previous knowledge about secure online behaviour and whether they were 
instructed by their parents or at school on ways to protect their privacy online.

We tabulated responses to our usage questions in Table 3.1. Most children used tablets and computers, primarily for playing games, doing school homework, checking e-mail, and watching videos. Most participants reported regular use of Youtube and various game sites to play online games or learn more on how to play a particular game. They also watch tutorial videos of other players playing the game on Youtube. Minecraft and Clash of Clans were the most popular games. Participants reported visiting Youtube for other various reasons, among them watching DIY projects and listening to music. Checking email was the third most popular activity reported by participants; Gmail was most popular.

All participants reported having passwords for various devices and accounts. While 10 reported sharing their password(s) with one or both their parents, some reported sharing their passwords with their siblings as well. Most participants mentioned chatting with their friends online. Google Hangouts was popular, along with iMessage. All participants mentioned that it is not good to chat with strangers and that they would refrain from doing it if they were placed in the situation. We gave participants categories of personal data and asked if they would share this type of information online. The majority agreed that it is okay to share some general information online, but that they should refrain from mentioning self-identifying information. A few mentioned that they would absolutely not share any personal information, while one participant mentioned that it is okay to share the name, age, favourite colour, and their daily happenings online. The majority of participants had a general idea of what personal privacy might mean. However, they merged it with online security, mentioning installing firewalls and not sharing their passwords online. Lastly, we asked if they had been instructed on safe online behaviour. Approximately half of participants have had such a discussion with their parents or at school.

\subsubsection{Pre- and Post-session Interview}

We coded participants' answers to the pre-and post-session questions with 3 for an excellent answer, 2 for a marginal answer, and 1 for a poor answer describing ways to protect online privacy. A excellent answer included advanced ways the participants 


\begin{tabular}{|c|c|c|}
\hline & & $\begin{array}{l}\text { No. of Partici- } \\
\text { pants }\end{array}$ \\
\hline \multirow[t]{4}{*}{ Devices used } & Computer/Tablets & 10 \\
\hline & Laptops & 9 \\
\hline & Smartphones/Ipods & 5 \\
\hline & PS3 & 1 \\
\hline \multirow[t]{8}{*}{ Main uses } & Playing games & 12 \\
\hline & Homework & 7 \\
\hline & Email & 7 \\
\hline & Watching videos & 5 \\
\hline & Texting/chatting with friends & 3 \\
\hline & Listening to music & 3 \\
\hline & Reading books online & 1 \\
\hline & Social media & 1 \\
\hline \multirow[t]{9}{*}{ Favourite sites } & Game sites & 9 \\
\hline & Youtube & 6 \\
\hline & Minecraft & 5 \\
\hline & Other: BBC, Online language courses, & 5 \\
\hline & Clash of Clans & 4 \\
\hline & Social media: Facebook, Instagram, & 2 \\
\hline & Pinterest & \\
\hline & Netflix & 2 \\
\hline & Gmail/others & 1 \\
\hline
\end{tabular}

Table 3.2: Participants' device usage, main uses and favourite sites. 


\begin{tabular}{|c|c|c|}
\hline & & $\begin{array}{l}\text { No. of Partici- } \\
\text { pants }\end{array}$ \\
\hline Password practices & $\begin{array}{l}\text { Have a password protected ac- } \\
\text { count(s) } \\
\text { Share password with parents, } \\
\text { and/or siblings }\end{array}$ & $\begin{array}{l}* 15 \\
10\end{array}$ \\
\hline $\begin{array}{l}\text { Online Privacy Under- } \\
\text { standing }\end{array}$ & $\begin{array}{l}\text { Understand what online privacy } \\
\text { is } \\
\text { Chat with friends online } \\
\text { Have had a discussion with an } \\
\text { adult on ways to protect online } \\
\text { privacy }\end{array}$ & $\begin{array}{l}13 \\
9 \\
7\end{array}$ \\
\hline $\begin{array}{l}\text { Sharing Personal In- } \\
\text { formation Online }\end{array}$ & $\begin{array}{l}\text { Okay to share some personal info } \\
\text { with people online } \\
\text { Not okay to share personal info } \\
\text { with people online } \\
\text { Okay to share personal info with } \\
\text { people online }\end{array}$ & $\begin{array}{l}9 \\
5 \\
1\end{array}$ \\
\hline
\end{tabular}

Table 3.3: Participants' password practices, and their understanding of online privacy. * Missing data for P5 due to technical error.

protect themselves online. For example, P7 mentioned how she is careful not to reveal her full name, and how she uses a fake name for her Instagram account. A marginal answer covered basic ways the participants protect themselves online. For example, P6 explains that in order to protect your online privacy, you make a very hard password that is hard to guess, you should use multiple passwords for different accounts. A poor answer showed a lack of knowledge of the basic ways to protect their online privacy. Figure 3.4 shows that half of participants displayed similar levels of knowledge before and after playing JCJF. The other half increased their knowledge of online privacy.

\subsubsection{Pre- and Post-scenario Questionnaires}

We focused on one repeated multiple choice question per scenario. On five out of the 12 scenarios, participants answered correctly on both instances (see Figure 3.5). Two scenarios showed an increase in correct answers. However, performance decreased on three scenarios; it is unclear why this happened. For example, one quiz question 


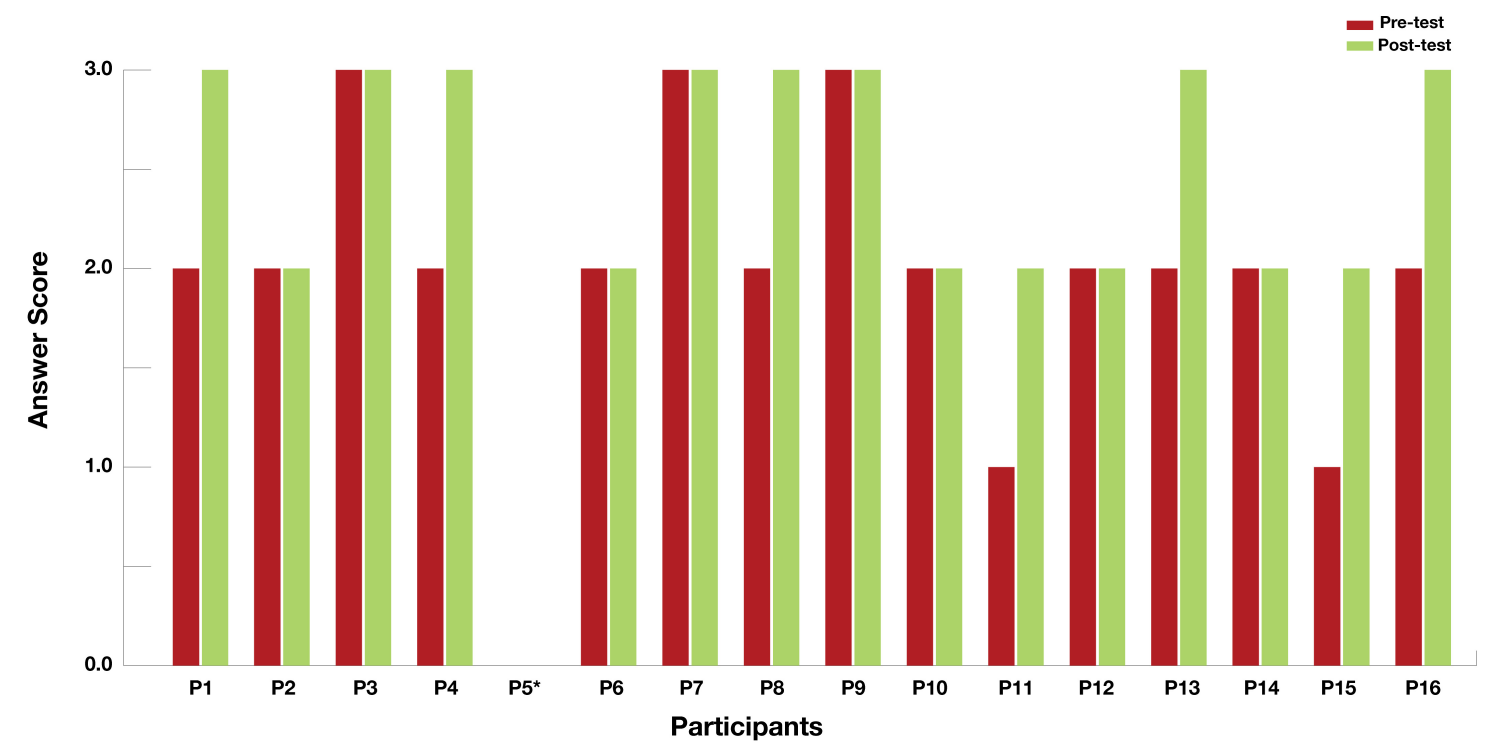

Figure 3.4: Scoring of answers for the pre-and-post test questions on online privacy $1=$ poor, $2=$ marginal, and $3=$ excellent. ${ }^{*} \mathrm{P} 5$ 's audio recording was lost.

asked for reasons why the Internet is so appealing to marketers. The choices included that it is popular with kids and teens, that the spending power of kids and teens is worth billions of dollars, and all of the above. Two participants tested this scenario and initially chose the right answer (all of the above). However, one subsequently chose just one option.

\subsubsection{Reading Times}

Figure 3.6 illustrates the mean reading time for each participant. Averages ranged from 99 seconds to 378 seconds $(M=208, S D=82.62)$, illustrating significant variance in reading abilities. We found that participants' age generally influenced reading times: participants with the slowest reading times were between ages of 11 and 12, while most of the fastest readers were 13 years old. However, there are some exceptions; P7, who was the fastest reader, is 11 years old, and P8, who is 13 years old, fell within the middle range.

Figure 3.7 shows the mean reading times per scenario. Sc 2 and Sc 4 show the data for one participant only, as the second participant's (P5) screen recording data was lost due to a technical error. From Figure 3.7, we notice that Sc 8 has the 


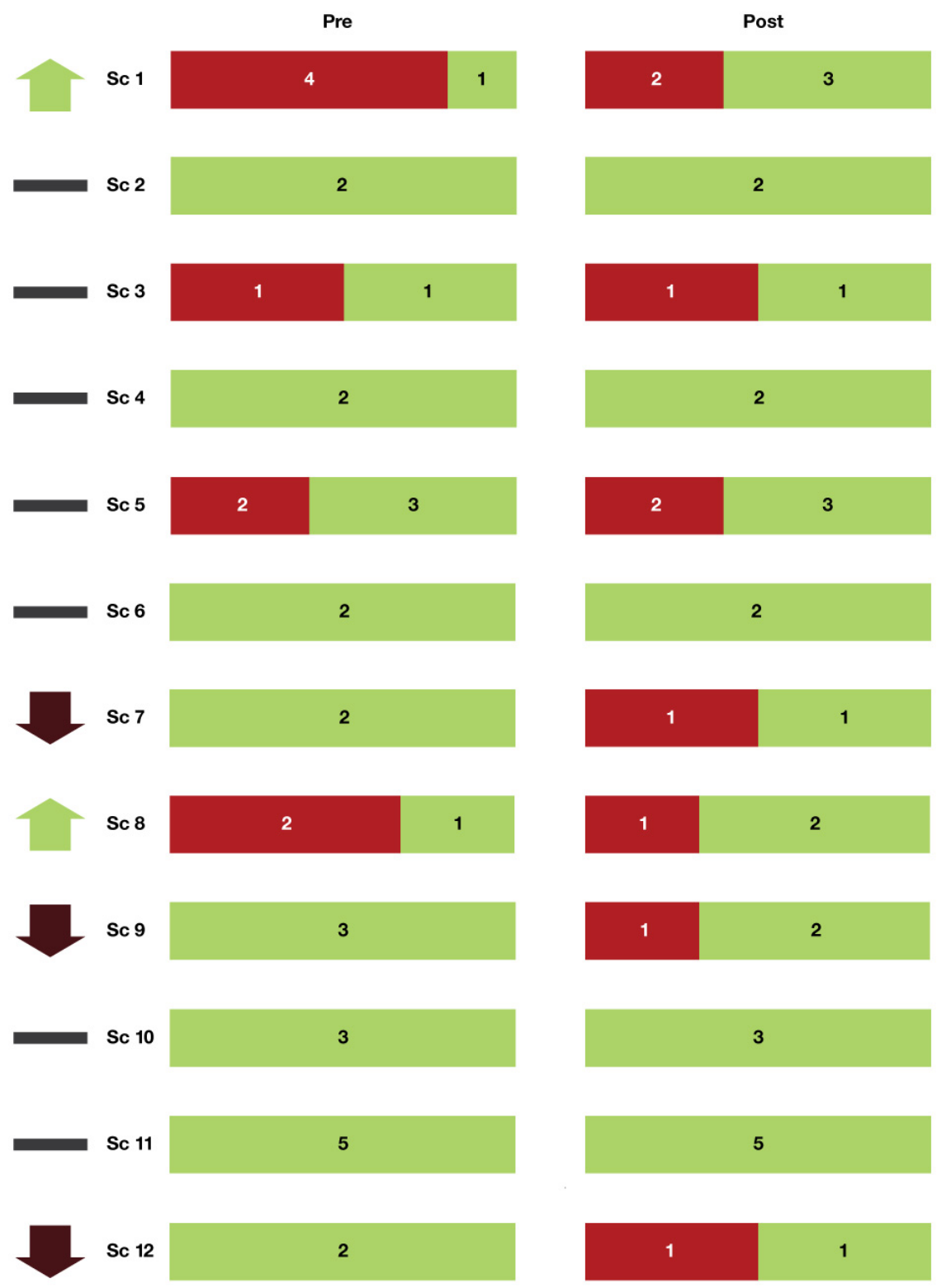

Figure 3.5: Pre-and-post scenario questionnaire responses. Responses were scored and the bar graphs represent the number of participants who answered the right/wrong answer. 


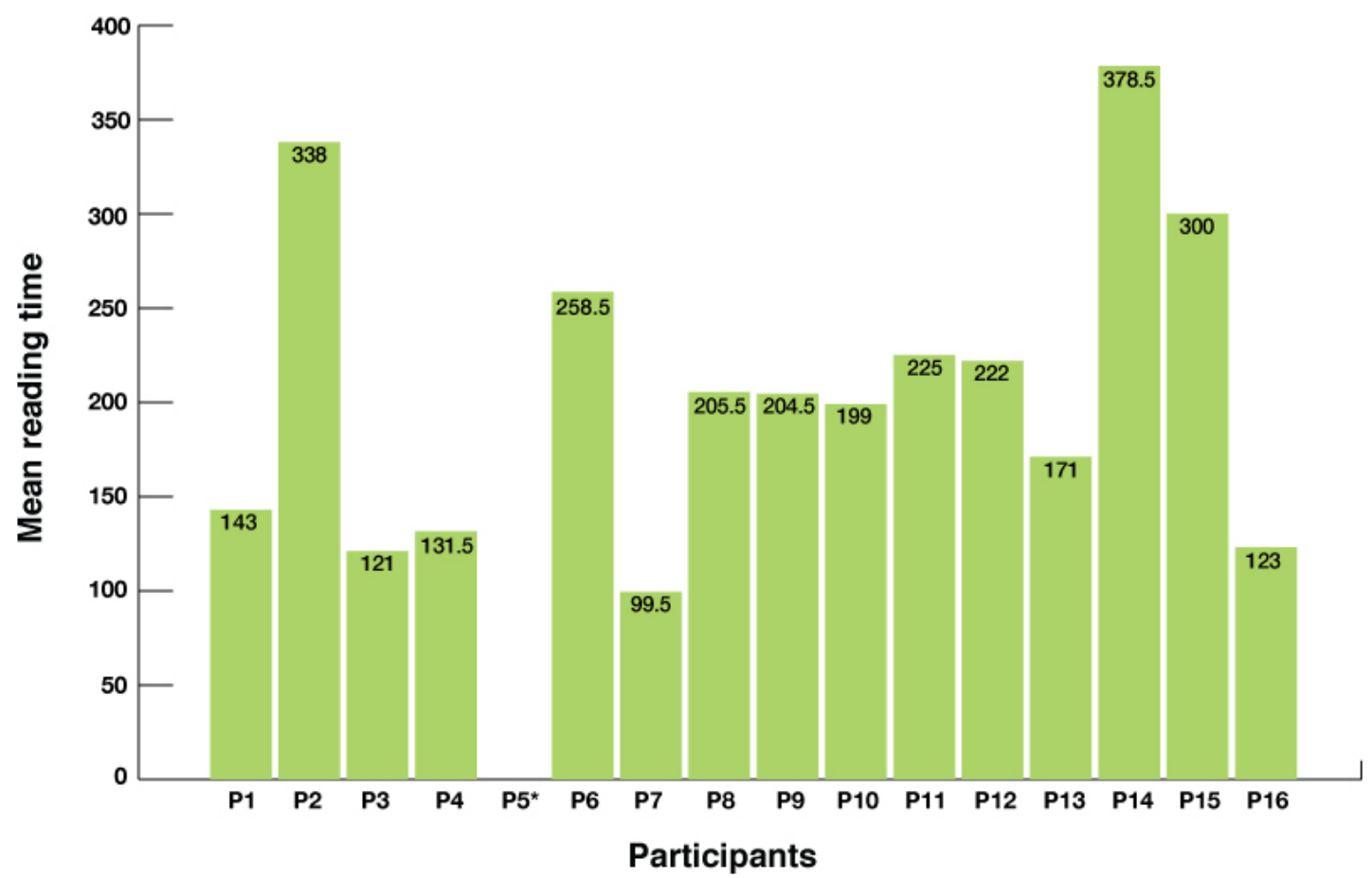

Figure 3.6: Participants' mean reading times per scenario in seconds. P5's data was not recorded. 


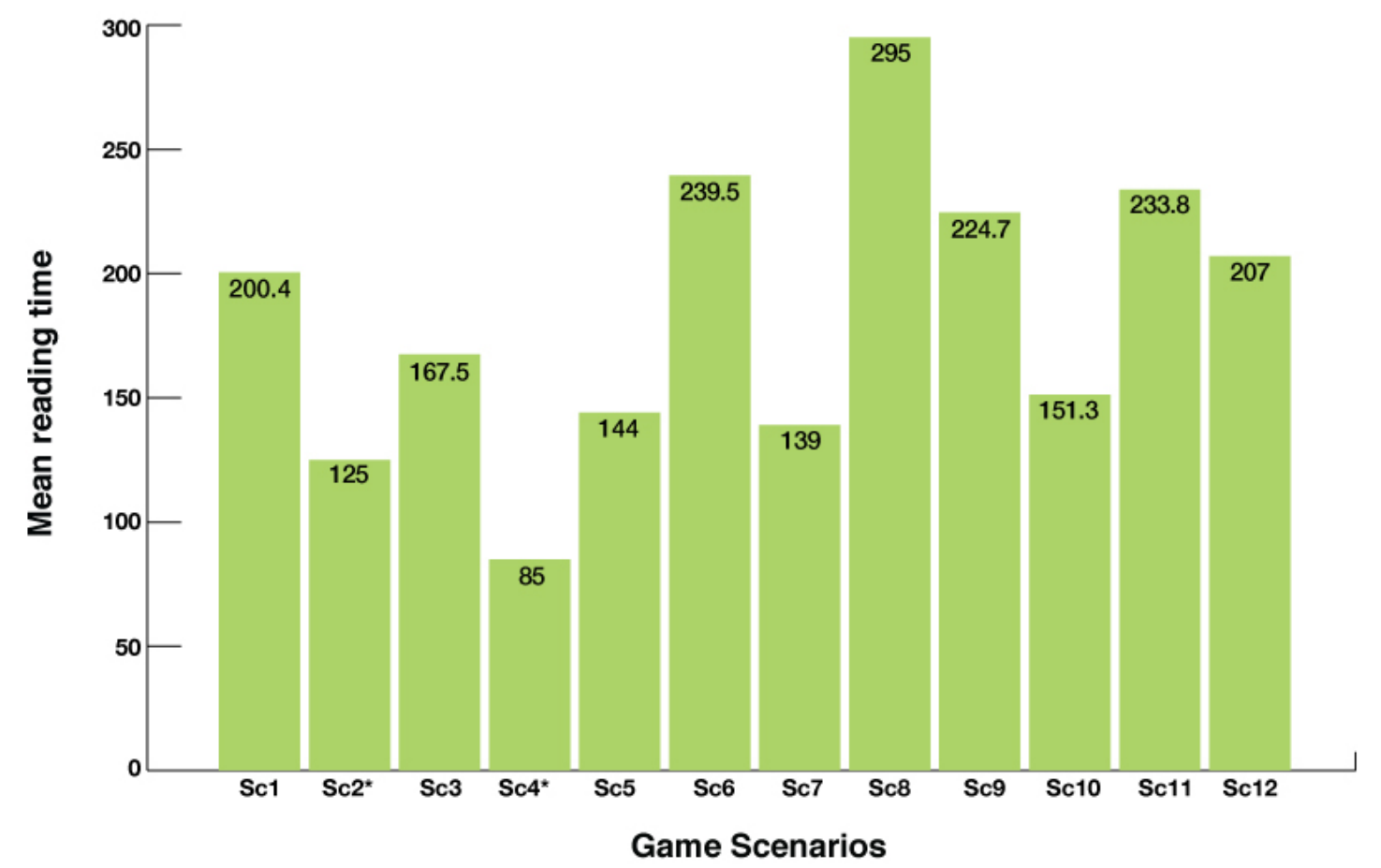

Figure 3.7: Scenarios mean reading times.

slowest reading time. Our observations suggest that participant variability had a larger impact than any inherent characteristic of the scenario; for example, the three participants (P6, P11, and P14) that tested with Sc 8 were simply slow readers overall.

\subsubsection{User Feedback}

Participants completed a user feedback questionnaire and provided verbal feedback during the session. Figures 3.8 through 3.14 show stacked bar graph plotting 5-point Likert scale responses ( $1=$ most negative, $5=$ most positive) to these questions evaluating their opinion of the game. We present these results contextualized with the participants' comments as they played the game.

Content length: Participants rated content length on a scale from Long to Short. Most participants rated the content as somewhat short $(M=3.56, S D=$ 0.62) (see Figure 3.8). This is in contrary to what we observed, especially as the younger participants read through the material. We noticed that they were hesitant or not as excited to read one paragraph of text after another. One participant (P14) 
kept asking: "do I read this as well?" while going through the game. P2 mentioned she disliked that some scenarios were so long. She also suggested that some of the words be simplified. P3 noted that some content was displayed in a primitive way. For example, some scenario websites stated "Click here, to learn how to hack," or "Click here to access the latest gossip news.", which he found "dumb." P8 described how reading is not one of his strengths, and how the lengthy content was his least favourite game aspect. One of his recommendations was to make the text a little bit shorter so that he would be able to read it better. Lastly, P4 also stated that showing less text would improve the game.

Game scenarios: Participants rated the scenarios on a scale from Boring to Interesting. Figure 3.9 shows that most of the participants found the scenarios interesting $(M=3.87, S D=0.88)$. They liked that the scenarios depicted real situations for real people. P4 mentioned that her favourite portion of the game was the scenarios. P8 also liked learning details of what is dangerous online behaviour and its consequences. He said that he learned quite a lot through the game scenarios.

Game play: Participants rated the play on a scale from Boring to Exciting. As shown in Figure 3.10, most participants were neutral with respect to their game play experience $(M=3.43, S D=0.81)$, with one third of participants responding more positively. P6 in particular mentioned how she liked playing the game and she was excited to learn about the different game scenarios.

Amount of learning: Participants rated how much they learned on a scale from Nothing to A lot. One quarter of participants self-reported that they learned a lot from playing the game; the rest chose either neutral or negative options $(M=3.03$, $S D=1.04$ ) (see Figure 3.11). P6 mentioned that he learned a few things, but when asked to specify, he could not remember what they were. P8 noted that he did learn something new in Sc 12, when Jo's friend received a low grade for plagiarizing his essay.

Game fun: Participants rated the game on a scale from Boring to Fun. 11 participants chose the somewhat fun and fun options $(M=3.62, S D=0.95)$ (see Figure 3.12). From the post-game interviews, participants liked the game scenarios, and found their details fun. 


\section{Content Length}

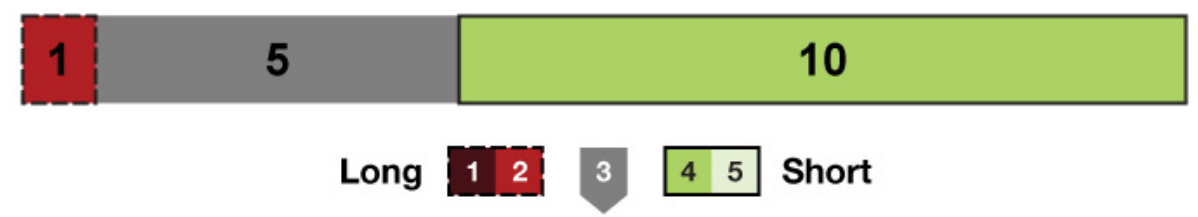

Figure 3.8: Likert scale responses for "The content was Long to Short".

Game Scenarios

\begin{tabular}{|l|l|l|l|}
\hline 1 & 4 & 7 & 4 \\
\hline
\end{tabular}

\section{Boring \begin{tabular}{llllll|l|l|l|l|l}
\hline 1 & 2 & 3 & 5 & Interesting
\end{tabular}}

Figure 3.9: Likert scale responses for "The game scenarios were? Boring to Interesting".

Game design: Participants rated the visual design of the game on a scale from Ugly to Nice. We found that most of the participants were not in favour of the game design $(M=3.62, S D=1.08)$ (see Figure 3.13$)$. They particularly did not like the design of some of the "fake" websites encountered. For example, P1 mentioned that she did not like the Splurge Cola website from the Sc 1 (see Figure 3.1). P3 suggested that we make the game look a little newer. He also commented on how the game displays on a small portion of the screen; he would like it to fill the whole screen and adjust from one screen size to another.

Recommend game: Participants indicated whether they would recommend the game to friends on a scale from Never to Absolutely. Figure 3.14 shows that 11 participants chose "Absolutely" $(M=3.87, S D=1.14)$. However, when verbally asked if they would recommend the game to their friends several were unsure (e.g., P7, P16). 


\section{Playing The Game}

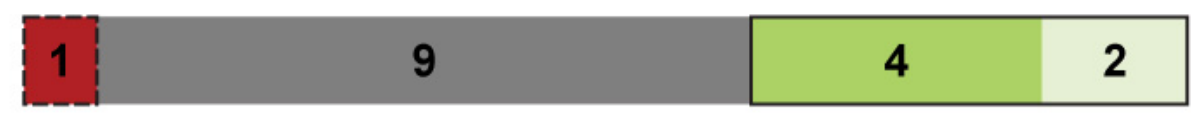

\section{Boring \begin{tabular}{ll|l|l|l|l|l|l|l|l}
1 & 2 & 3 & 5 & Exciting
\end{tabular}}

Figure 3.10: Likert scale response for "Playing the game was? Boring to Exciting".

\section{Amount of Learning}

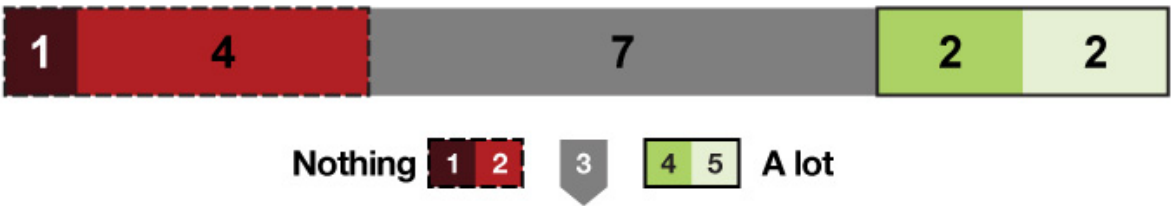

Figure 3.11: Likert scale responses for "How much did you learn? Nothing to A lot".

\section{Game Fun}

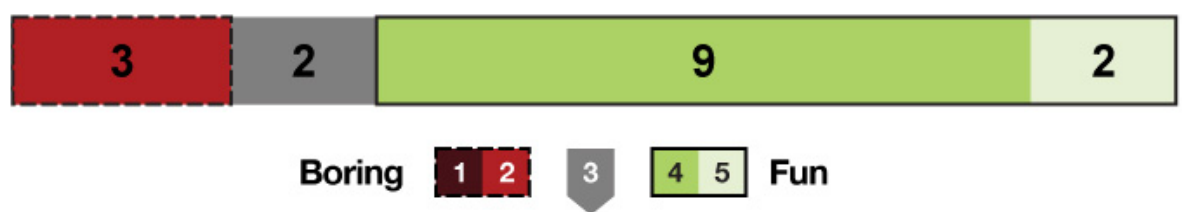

Figure 3.12: Likert scale responses for "The game was? Boring to Fun".

\section{Game Design}

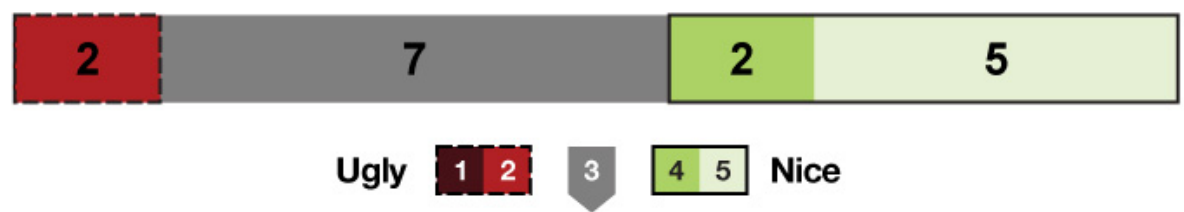

Figure 3.13: Likert scale response for "The game design was? Ugly to Nice". 


\section{Recommend Game To Friends}

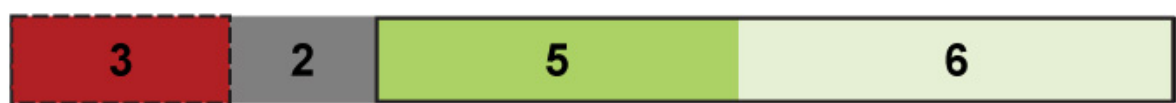

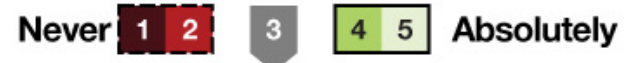

Figure 3.14: Likert scale response for "Would you recommend this game to a friend? Never to absolutely".

\subsubsection{Eye Tracking Data Analysis}

Using the eye tracking data, we analyze if participants were drawn to certain elements of JCJF. We explore whether colours, pictures, and animations caught their gaze. Finally, we examine the reading patterns. We manually examined each participant's gaze through the eye tracking recordings.

Using the eye tracker, we were able to capture the participants' gaze. Data was then analyzed through the heat mapping function provided by the eye tracking software and translated into a coloured map that appears on top of a still image. The colours range from red to orange to yellow to green represent the areas where the participant gazed most to least, respectively.

Scan paths also track the participants' gaze to the different locations around the screen. Larger circles represent more time spent continually gazing at that area. The dots are connected with a line showing the order of where the participant looked on screen.

Faces: Participants were drawn to the faces of Jo and Josie through out the game, especially when the Jos were making eye contact with the camera. Figure 3.15 shows a heat map of P8's screen gaze. The heat map illustrates how Jo attracted as much attention as the text. Figures 3.16 and 3.17 show scan paths for P1 and P6. They illustrate how Josie's face is getting a fair amount of attention as the participant reads the text. Participants focused on reading when either Jo or Josie had their eyes almost closed or averted, as illustrated in Figure 3.18. Similarly, Figure 3.19 shows the game's landing page with Jo and Josie looking at each other, and the player barely glances at them. In both cases, Jo and Josie's eyes are either closed or looking away 


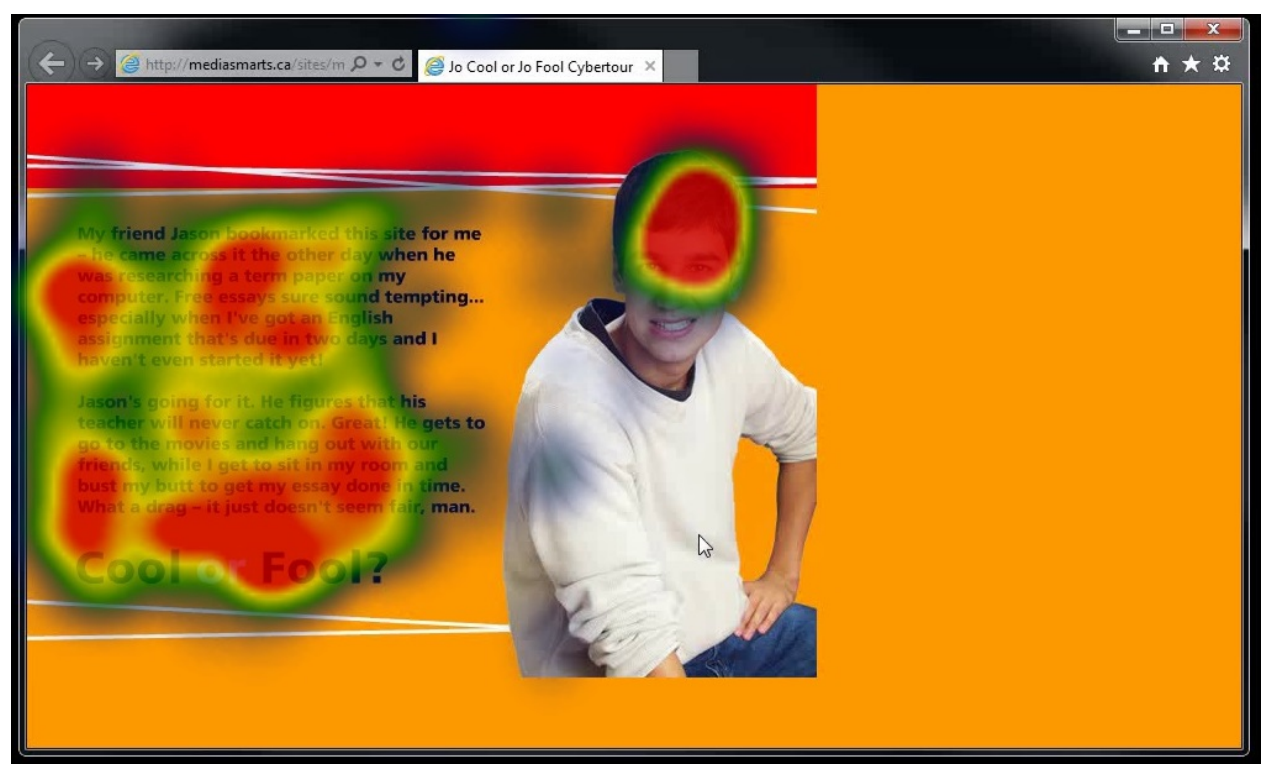

Figure 3.15: Heatmap showing a participant's fixation on Jo's face.

from the players, which makes the players less compelled to look at them.

Text styles and Layout: Text styles and layout played a major role in the design of JCJF. Figure 3.20 shows how the title "cheapmail.com" and the slogan underneath introduced a hierarchy to the page. P15 then notices the navigation links underneath the title and immediately moves to the "What's Jo thinking?" button. Here, the participant's gaze was guided by the designed text hierarchy. We note that the large block of explanatory text was mostly ignored as the participant made sense of the page through other design elements

Game navigation: The game navigation was sequential, with a single link that either changed colour on hover, or blinked (see Figure 3.1b). In general, participants were annoyed that although most of the scenarios looked like real websites, they were not active. Sc 5 was particularly frustrating to participants. Figure 3.21 shows the scan paths of P2 who unsuccessfully clicked on links within the website because they expected it to behave like a real website. Sometimes the actual link was not obvious, like the one found in Sc 11 (Figure 3.22). The active link is "schools" link on the right hand side of the screen, located in the middle of what looks like the website's menu. Even though it was blinking, we noticed that the participants required multiple attempts to find the right link. 


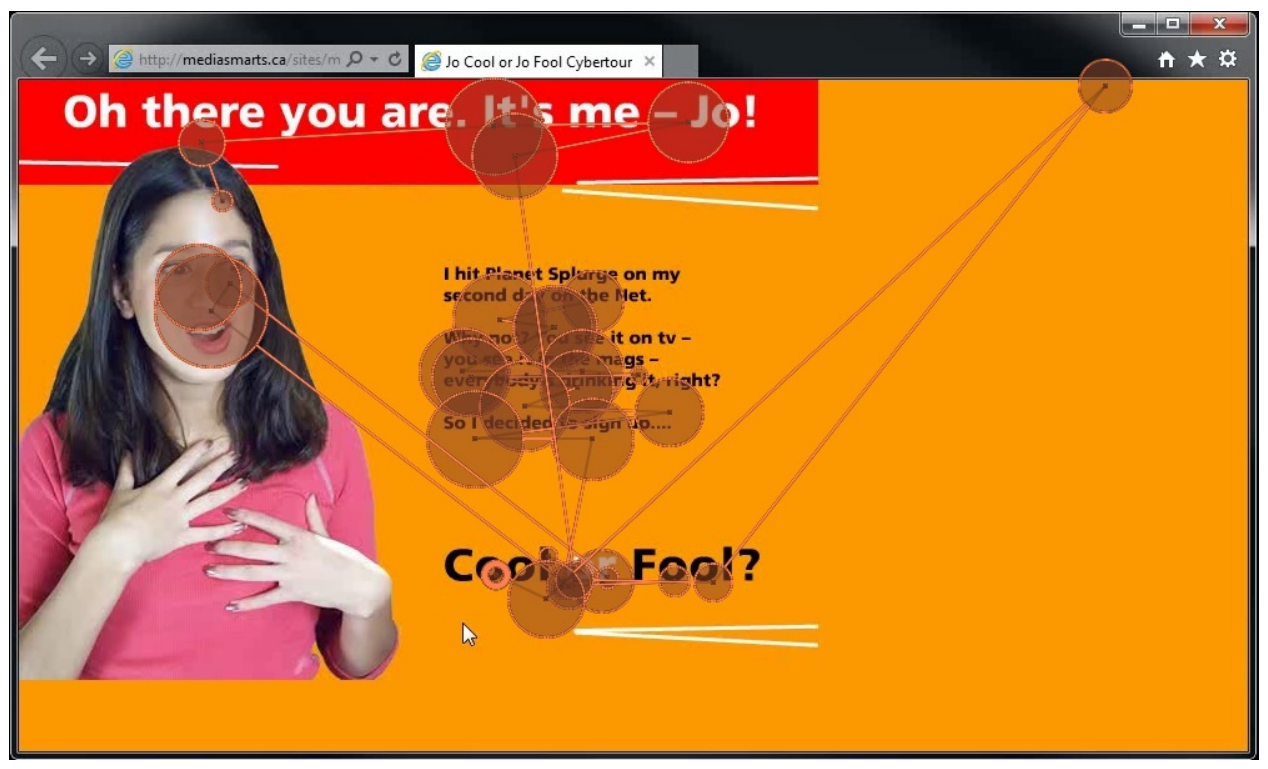

Figure 3.16: Scan path showing a participant's fixation on Josie's face.

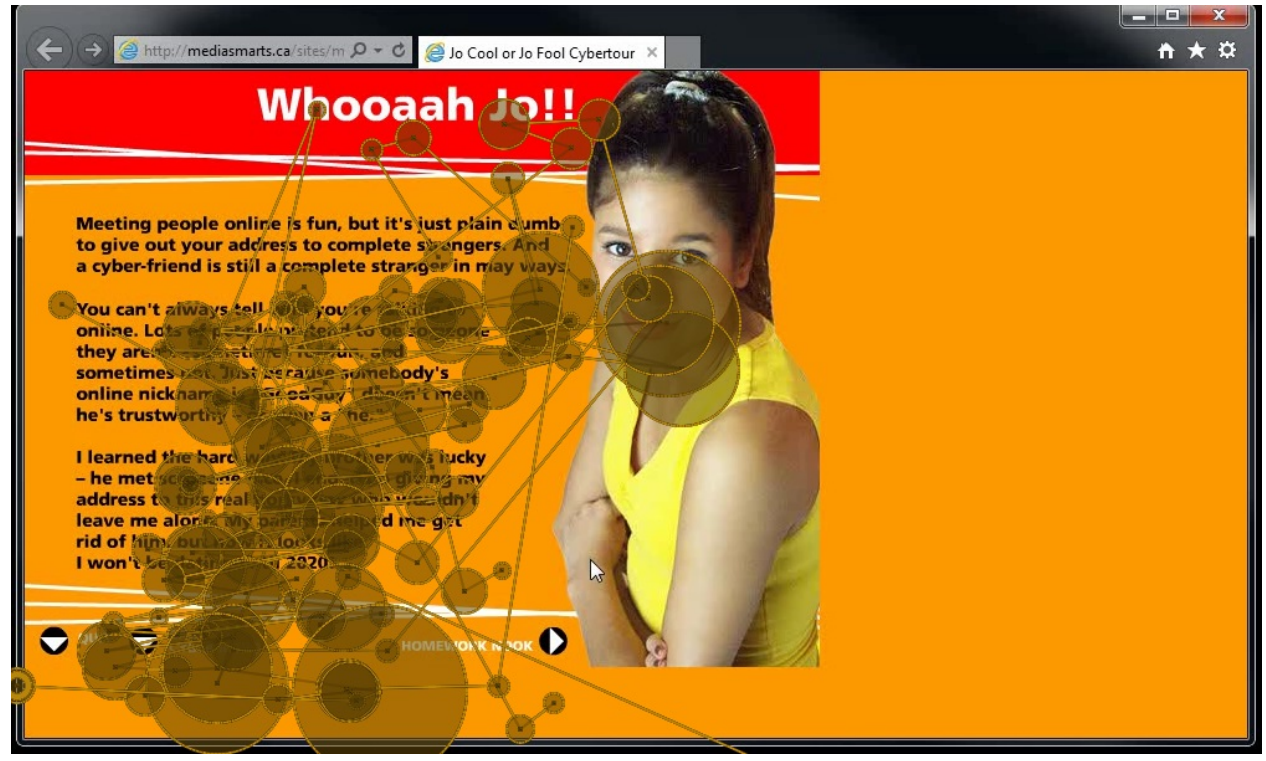

Figure 3.17: Scan path showing a participant's fixation on Josie's face following a zig-zag reading pattern where they read some, look around, and read some, and look around again. 


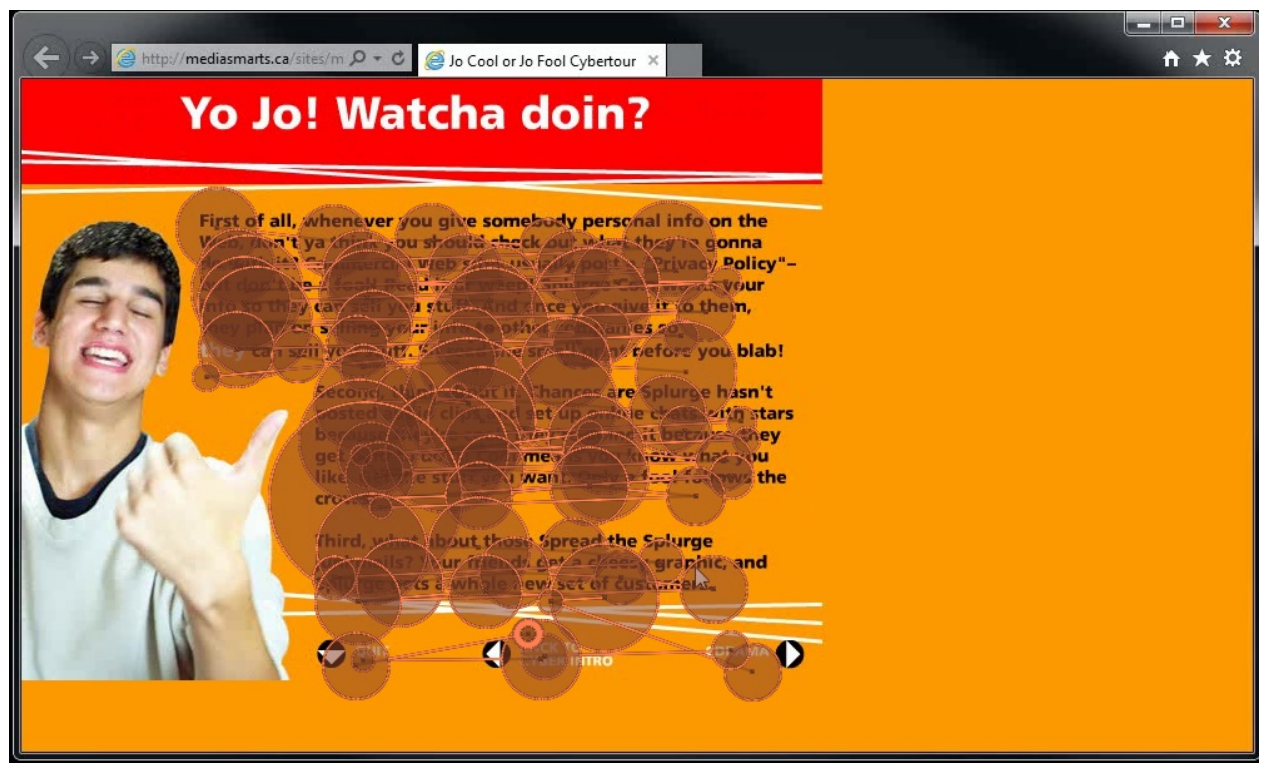

Figure 3.18: Scan path showing a participant's fixation on the text alone without referring to Jo's face, following a line-by-line reading pattern

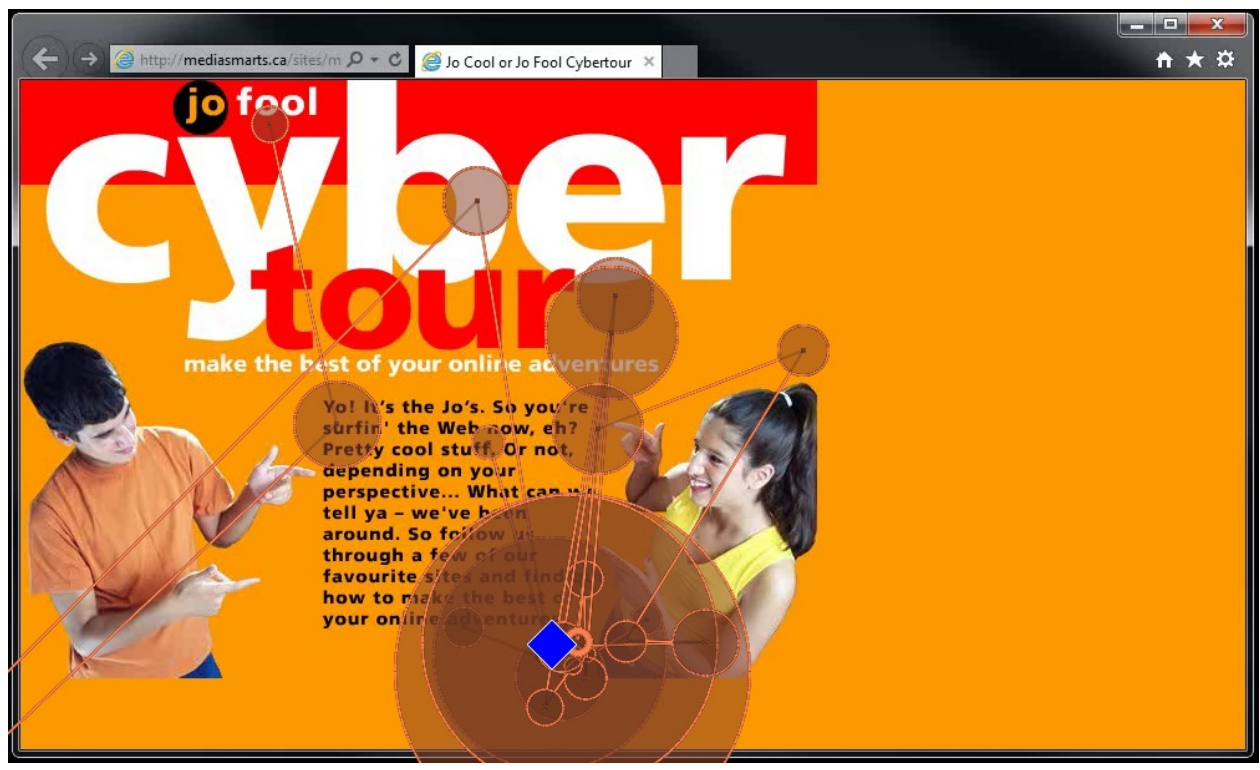

Figure 3.19: Scan path showing a participant's fixation on the forward arrow to move them to the next screen, while ignoring the Jos who were not making eye contact with the camera. 


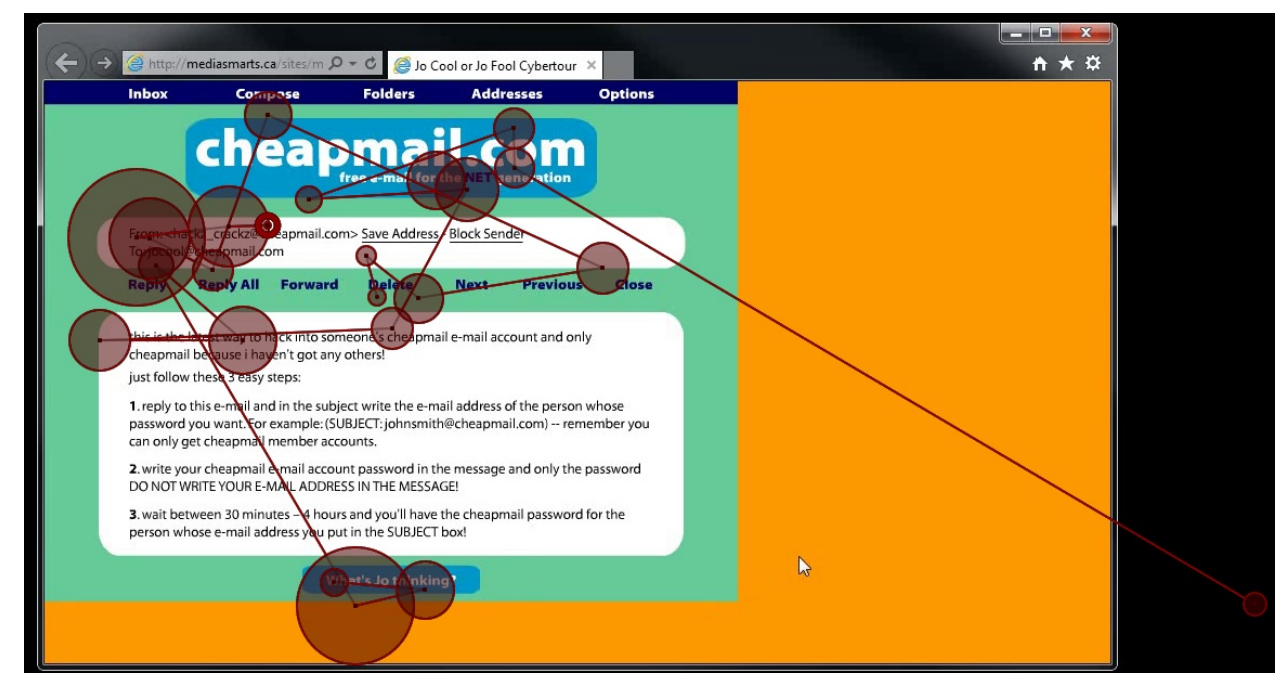

Figure 3.20: Scan path showing a participant's fixation of the Cheapmail scenario. Participants were drawn to the title and the coloured button at the bottom of the screen. They spent time exploring the navigation buttons at the top, but skipped the plain text treatment of the body of the email.

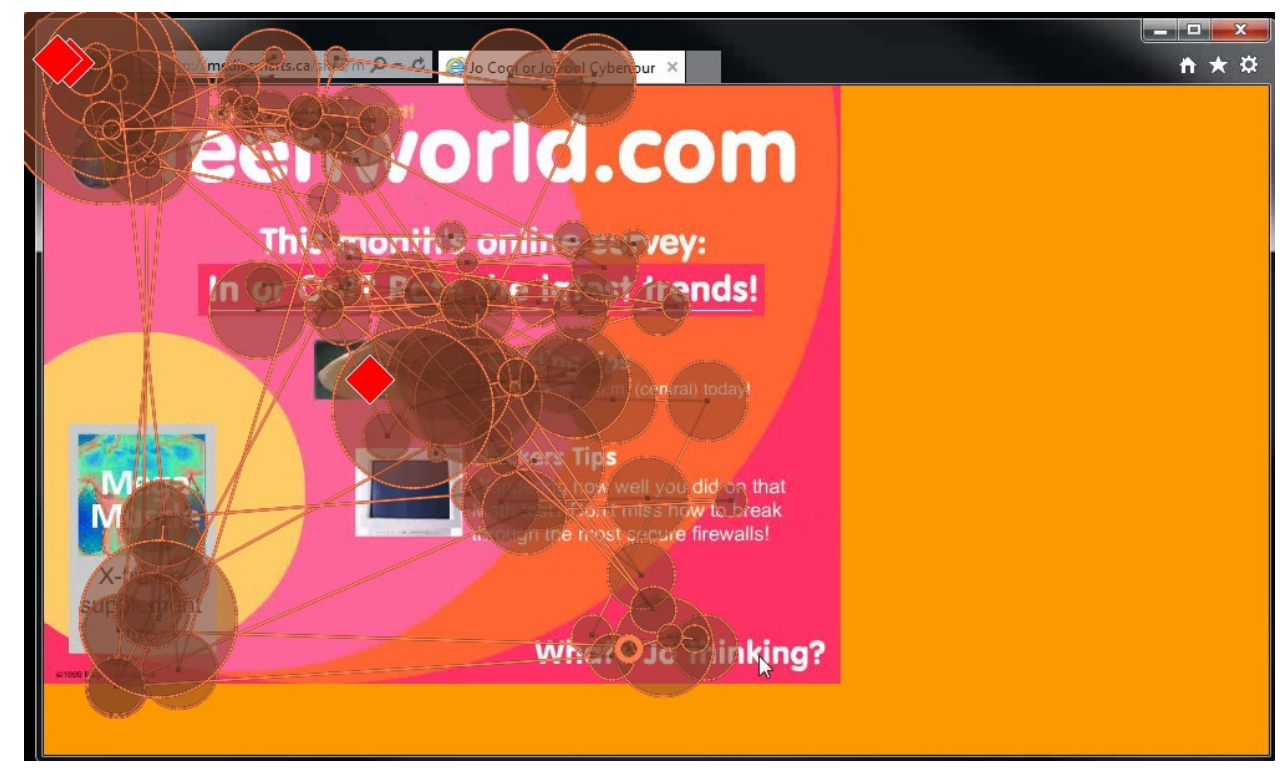

Figure 3.21: Scan path showing a participant's fixation of the Teenworld.com scenario. Scan path shows how participants spent considerable amount of time trying different link options but were not successful as the Teenworld.com site was not active. The red diamonds represent the participant's clicks. 


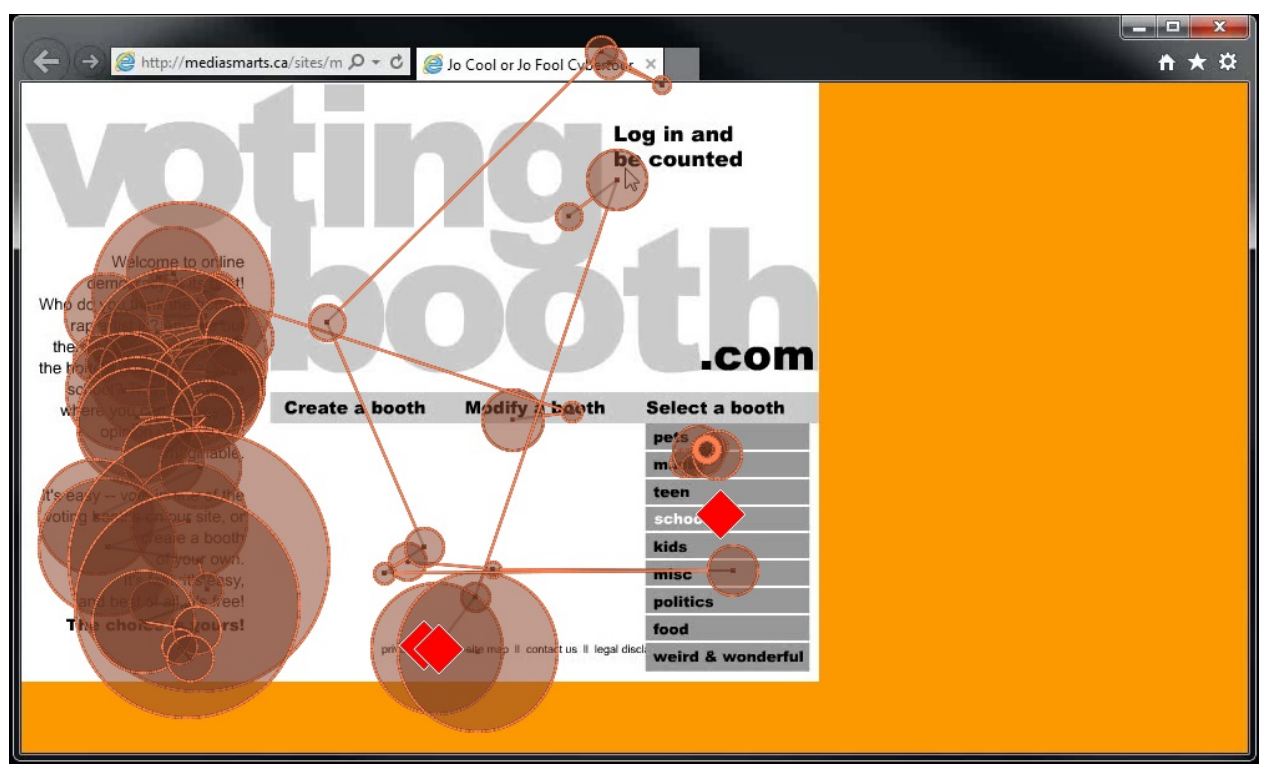

Figure 3.22: Scan path showing a participant's fixation on the left hand side tab trying to find out the navigation to the next page. Scan path shows how participants gazed at other links on the left hand side of the screen, while not noticing that only the "schools" link that blinked in red is active.

Reading patterns: Line-by-line: Most participants read the game scenarios line-by-line, as demonstrated in Figure 3.18. Next, they located the navigational arrows to figure out the next step.

Zig-zag: Some participants followed a zig-zag reading pattern in the sense that they would read, drift their gaze to another object on screen, then come back to reading, as illustrated in Figure 3.17. The zig-zag pattern was particularly evident in slow readers who frequently asked if they had to read dense text (e.g., P14). In some instances, participants started with a zig-zag, read line-by-line, zig-zagged a few times in the middle, then continued line-by-line until the end. Figure 3.23 illustrates such a mixed reading pattern.

Skimming: The game contained dense text areas. Sometimes, participants skipped reading certain screens altogether. Figure 3.24 illustrates how P9 skips from the beginning of the page to the bottom while reading only a few words in the middle. This particularly happened in Sc 10 as it had the densest text. 


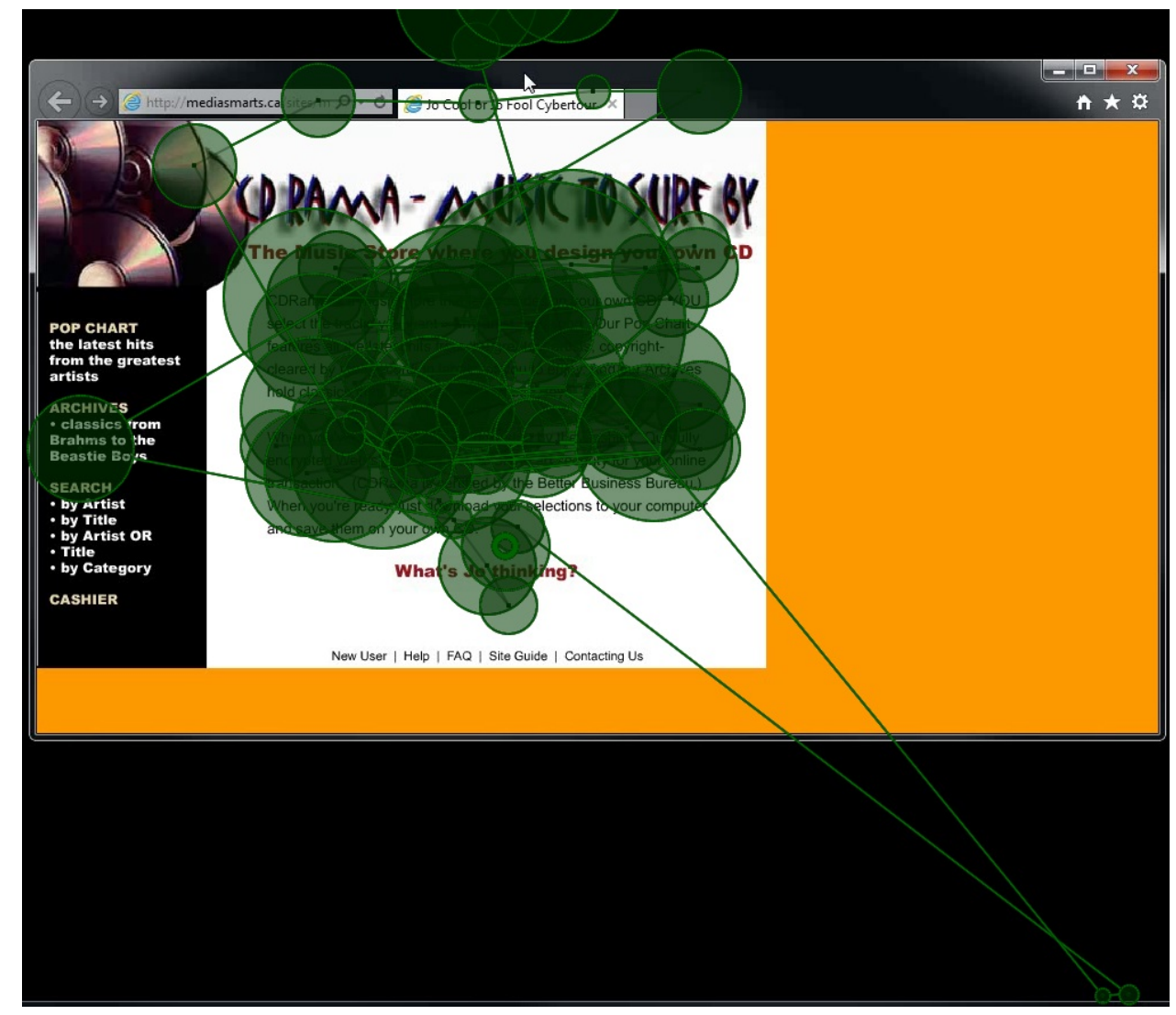

Figure 3.23: Scan path showing a participant's line-by-line and zig-zag reading patterns. Participants some times relied on such mixed reading pattern to take a break from dense text areas. 


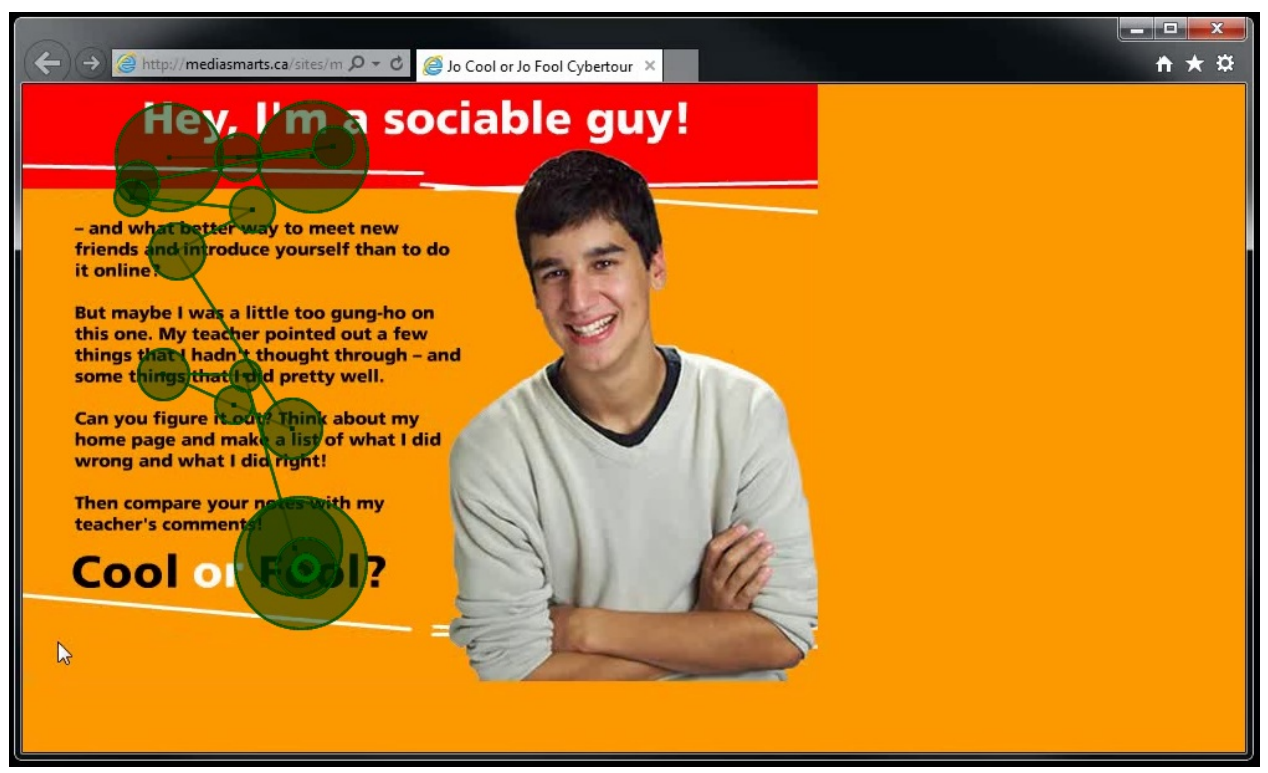

Figure 3.24: Scan path showing a participant's skimming over text areas. This screen came as the 4th page of the long Sc 10 (see Figure 3.2j-l).

\subsubsection{User Feedback Questionnaire}

The following section discusses the participants' answers to what they liked, disliked, and recommended as improvements.

Likes: Participants generally liked the game. For example P6 said: "I like the game and I wanna play more of it." They appreciated the real-life scenarios, and how the scenarios gave detailed description of what could happen based on the Jos' choices (P4 and P8). Some participants appreciated advice on identifying a malicious website or situation and felt that they learned a lot from it (P1, P3, and P14). They enjoyed choosing whether certain actions were "cool" or "fool" by identifying whether this was a good or bad situation (P2 and P16). P10 appreciated how the roles were equally split between a boy and a girl, and P12 liked that Josie was the one correcting Jo's actions. P11 liked how JCJF forced them to think about the different scenarios and make a choice, while P16 commented "I like how the game didn't tell you everything, and you have to think about what you're reading and not just browse through it." Lastly, participants appreciated the variety of scenarios.

Dislikes: Overall, the participants were really hesitant to mention what they disliked about the game. However, P15 mentioned that he probably would not have 
played the game if it was not for the sake of the user study. P1 did not like the look and feel of Sc 1. P3 thought all the websites in JCJF seemed really "dumb", and included dumb statements like "Learn how to hack here!" (Sc5). P10 questioned whether the two scenarios she tested were age-appropriate and thought that they introduced wrong ideas to young children like herself. For example, she noted that Sc 12 introduced the idea of plagiarism to young children, "Now, I know that I could find essays online." She thought that Sc 3, introduced the concept of online dating to an audience that is much too young like herself and that this should be introduced to 18 year olds. P10 similarly kept repeating: "huh, I thought online dating is for older people, like 30 year olds."

The length of the scenarios was a main concern for several participants (P2, P4, and P8). We observed other participants also frustrated with the length while reading the scenarios. P14 repeatedly asked, "do I have to read this one too" for every new page.

Things to improve: Some participants articulated clear recommendations for improvement. P1 suggested showing example sites that are safe and others that are malicious and asking players to tell the difference between them. P2 suggested changes to some of the wording, as she found some text unclear. P3 wanted to increase the difficulty by making the correct options less obvious, and he wanted to make the game look a little newer. P4 and P8 suggested larger text that is shorter and more fun to read. P8 mentioned that he preferred manipulating things rather reading about them. P11 reiterated concern about the age appropriateness of the two game scenarios she played. She mentioned that the game should be better suited for an 11 years old.

\subsection{Summary}

In this chapter, we tested JCJF with 16 participants to identify areas of weakness and strength. Results show the participants liked certain aspects of the game and disliked others. They liked the fact that the game is scenario based and shows a brother and sister. They disliked the fact that the scenarios and the overall lookand-feel of the game is outdated. Some participants thought scenarios like online 
dating (Sc10) were not appropriate for their age group, and deemed it for older teenagers, like 18 and older. Eye tracking data showed the participants were drawn to certain design aspects, like faces. Participants disliked the dense areas of text throughout the JCJF scenarios. This appeared in both their verbal comments and their reading patterns. Verbal comments like: "do I read this as well?" were repeated often, especially in dense areas of text. Skimming and zig-zagging were two of the main reading patterns that participants relied on when faced with large content to read. Participants' questionnaire responses were in favour of recommending the game to their friends, however, when verbally asked, they mentioned that they probably would not recommend it to any of their friends or spend time playing it if they stumbled across the game outside of the user study. In the next chapter, we explain the redesigned game where we combine this study's results and the participants' feedback in designing the new game. 


\section{Chapter 4}

\section{Design of the new game: "A Day in The Life of the Jos"}

Building on the findings of the previous user study, we devised a new direction for the existing game. Following the ADDIE instructional design model, we iteratively designed a new game called "A Day In The Life Of The Jos" (ADITL). We identified our typical end-user, the environment where the game will be implemented, and game elements that will appeal to our age group. The game needs to include educational elements to ensure the learning [8]. In the following sections, we explain the redesign process, the new game prototype, the design objectives, the analysis of the prototype, and the selected game mechanics.

\subsection{The ADDIE instructional design model}

According to Gustafson and Branch [28], instructional design is a method that helps in developing educational material in a uniform way. $\operatorname{ADDIE}^{1}[28]$ is a framework to guide the process of developing instructional material. The 5 phases of the ADDIE model are: Analysis, Design, Development, Implementation, and Evaluation. The ADDIE model was first built as a waterfall process [6], where each phase had to be fully completed before initiation of the next phase. However, this process has evolved to be iterative [28]. Figure 4.1 shows the latest version of ADDIE and the relationship between phases. Here are the 5 phases in greater detail:

Analysis: The designer identifies the instructional problem to be solved and outlines it by exploring the targeted audience, their current knowledge, and the desired learning goals. The designer also identifies any constraint issues and the delivery options.

Design: During the design phase, the designer transfers the learning goals from the analysis phase into different UI prototype options. Scenarios and content are

\footnotetext{
${ }^{1}$ acronym stands for the 5 stages of instructional design
} 


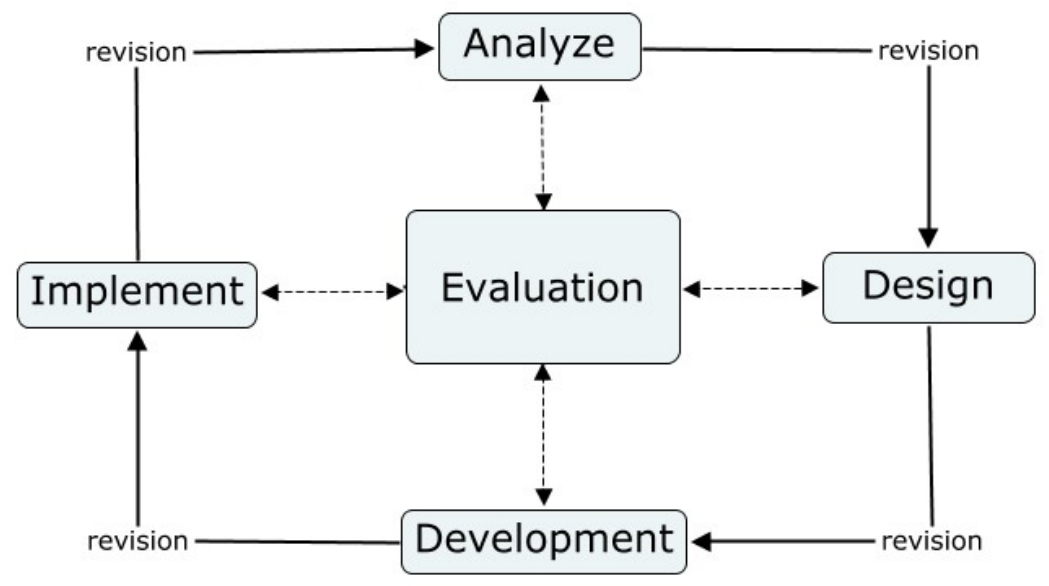

Figure 4.1: The 5 phases of the ADDIE instructional design model. Figure adapted from Wikimedia Commons [11].

mapped out, and anchored around the learning goals. The designer starts to generate the overall look and feel of the UI and defines the user experience according to the previously determined learning goals. Low-fidelity prototypes are generated to iteratively test the concepts and visuals. This is done to ensure the design meets the requirements of the target end-users (both learners and educators). Constraints highlighted in the Analysis phase are addressed and solved.

Development: The designer and developer work together to create a mediumfidelity prototype of the educational material. Both the visual UI and back-end development are addressed. An iterative process is particularly important during this phase, to refine the prototype and ensure reaching the originally outlined learning goals.

Implementation: Once the designer has obtained a working version of the blueprinted design, the prototype is implemented and tested with real end-users. Through an iterative design process, the designer goes back and applies updates and fixes necessary based on usability testing. The implementation phase might have multiple rounds of testing and fixing issues to produce a finished or close to finished version of the UI. Testing is extremely important during the implementation phase because it ensures that the finished product is fully functioning and bug-free before 
deployment at the intended venues.

Evaluation: Formative and summative evaluations are two important aspects of this phase. Formative evaluations are carried out at every stage of the ADDIE process. Both quantitative and qualitative evaluations are utilized to gather feedback. The feedback is then incorporated in the design. Summative evaluation are normally performed on the finished version of the instructional product and they aim to measure the success at reaching the educational goals among the target audience.

\subsection{Analysis Phase}

Following the ADDIE instructional design model, we started with the analysis phase. We describe our choices and their reasoning in the following sub sections. We defined the design objectives for the new game. We identified the gamification elements that would encourage children to play ADITL for the duration of the game session. We identified the intended age group, since this will be the driving factor for the educational elements and the game components. We conducted several initial brainstorming and design sessions with MediaSmarts to determine the general direction of the game.

\subsubsection{Intended audience}

Our first goal in the game's redesign was to identify the new target age group, as we established in Chapter 3 that the age group (11 to 14 year olds) for JCJF is too wide. We narrowed the audience to 11 and 12 year olds with feedback from MediaSmarts.

\subsubsection{Educational goals}

After initial assessment of the existing game, we discovered that some of the online privacy and security topics of JCJF do not apply for this younger age group (e.g., online dating). In consultation with MediaSmarts, we settled on 5 topics to address in the new game. As educators with many years of experience in this space, we relied on MediaSmarts to make the final decision on what would be the proper behaviour for each in-game choice and its consequences. We define each topic and discuss their 
respective educational goals:

1. Cyberbullying: According to the general definition of cyberbullying [53], it is the act of bullying someone through electronic means (e.g., by posting mean or threatening messages about the person online). Rivers and Noret's study suggests an increase in cyberbullying in recent years [71]. We focused on teaching the children how to handle tricky situations like being a witness to someone being teased online. Other skills include learning to check the facts to avoid rumours, and assessing possible consequences of their actions before posting any content that can be used against others. They also learn to avoid contributing to cyberbullying, and different ways of remedying a bullying situation, whether online or offline.

2. Data mining: Data mining is the active harvesting and use of information from users by third-party sites without the knowledge of the user [83]. The educational goals for this topic include identifying tactics used by online marketers to gather personal information. The advice includes for children to avoid online registration forms requesting personal information to access "fun" areas of a site, to be wary of quizzes and surveys that are used to determine the preferences and attitudes of young consumers, and to avoid contests in which the children are required to submit personal information to enter or win prizes. Another scenario teaches children to be aware of chat environments where children may be asked for personal information in order to join a chat, or where children may inadvertently post personal information while chatting. Finally, the scenarios explain how marketers create customer profiles and deliver customized information to young visitors.

3. Internet privacy: Online privacy is the user's right to preserve their personal information from being shared or stored, or sold to third parties [3]. The educational goals for this topic include practicing secure behaviours such as careful use of passwords, logging out of accounts, and setting privacy controls. It also includes advice on appropriate content to post and avoiding accidentally revealing personal information. 
4. Image sharing: Photo sharing is the posting of personal and others' photos online. The overall message in this topic relates to understanding the ramification of sharing images and videos of others online. The educational goals include obtaining others' permissions before taking or using their photos, editing pictures (e.g., blur out faces) if sharing images of others without consent, and controlling who views the shared images.

5. Verification of information from online sources: Children frequently rely on online sources to research a topic for school assignments or personal knowledge. However, little effort is being placed in teaching children on how to verify the accuracy and ownership of the information they find online. Lessons focus on appropriate use of copyrighted material, checking sources of information, and cross referencing information through multiple sources.

\subsubsection{Entertainment goals}

According to Kapp [35], using game elements in an educational environment acts as a strong motivator for children to learn about new topics. Given that learning about privacy and security are not typically a primary goal [85], a main strategy for ADITL is to add fun and entertaining elements to increase children's engagement. Although JCJF is somewhat fun, it includes few game elements. There were no incentives encouraging children to explore the game.

\subsubsection{Conversation-provoking goals}

A third aspect of the analysis phase of the ADDIE process is to look at the context of use. JCJF is still in use within classrooms, and the new game will also be used within the same setting. At school, children might be interacting with the game individually or in groups. Either way, the game design should encourage conversation to encourage students to reflect on the content and relate it to their own experiences. 


\subsection{Design Phase}

From the JCJF game we decided to keep the two main characters, brother and sister, Jo and Josie, because participants of the JCJF user study appreciated seeing them interchangeably throughout the 12 game scenarios. Participants also liked the fact that JCJF is scenario-based. They liked following the Jos through the different scenarios and deciding whether their actions are cool or fool. For that reason, we kept ADITL scenario-based as well. However, scenarios in ADITL are tied with a calendar day, where each calendar day addresses a specific topic through five scenarios.

The ADITL topics are similar to JCJF's, teaching children how to protect their online privacy and security. We used feedback from the JCJF study and surveyed current issues in online privacy and security that pertain to the safety of children. We worked closely with MediaSmarts, the organization that released JCJF in early 2000s, concerning which topics to include. We divided the ADITL into 5 days, each addressing a different topic.

\subsubsection{Game topics}

Game Topics: Each topic/day has five different scenarios reflecting nuanced situations, where players have to carefully consider each option in order to make the best choice. Appendix A.8 includes the 25 game scenarios across the five topics.

The 5 days cover the following topics, which align with the educational goals from Section 4.2.2:

- D1: Cyberbullying

- D2: Data Mining

- D3: Internet Privacy

- D4: Image Sharing

- D5: Verification of Information from Online Sources 


\subsubsection{ADITL overview}

When testing JCJF, we noted that participants enjoyed helping Jo and Josie make the "right" decision. For the new game, we chose to follow the Jos through the events of their day, and having the player help the main characters make positive choices. From the main landing page, as shown in Figure $4.2^{2}$, the player can choose one of the five game days. Each day (Figure 4.3) contains a sequence of scenarios set at different times throughout the day. The player must complete the scenarios in chronological order; with subsequent scenarios available only once the preceding one is complete. The player starts by clicking on the first scenario, the only clickable box. This will trigger the scenario's first scene (Figure 4.4), followed by the closeup view (Figure 4.5), and finally ending with the choices view (Figure 4.6). After the player chooses an option the newsfeed tab will pop open reflecting the consequence of the choice on Jo or Josie's social media feed (Figure 4.7). At the end of each day (five scenarios covering one topic), an overlay screen appears, reviewing the player's choices and consequences as a summary for that lesson (Figure 4.8). From the calendar, the player can play other days, or end the game session.

Landing Page: The ADITL landing page is a calendar (Figure 4.2). Active calendar dates use red text to indicate that they contain scenarios, changing to pale yellow on a grey background on hover. Clicking on each active date leads to a zoomed in view of that particular day's scenarios.

Day View: Each day (Figure 4.2) is composed of 5 scenarios that happen sequentially at different times of day. Scenarios become active only once earlier scenarios are complete. Similar to the main calendar, an active time is coloured in red and sits on a dark grey background on hover. We currently have five active days, labelled D1 to D5.

Scenarios: The scenarios revolve around situations where the Jos are faced with tricky choices. The player has to help the Jos make the most appropriate decision. The scenarios are meant to train the children on how to identify these situations and how to use critical thinking to reach the most appropriate response. As an example,

\footnotetext{
${ }^{2}$ Figures in section 4.3 are from a later prototype and are used here to better illustrate the concepts.
} 


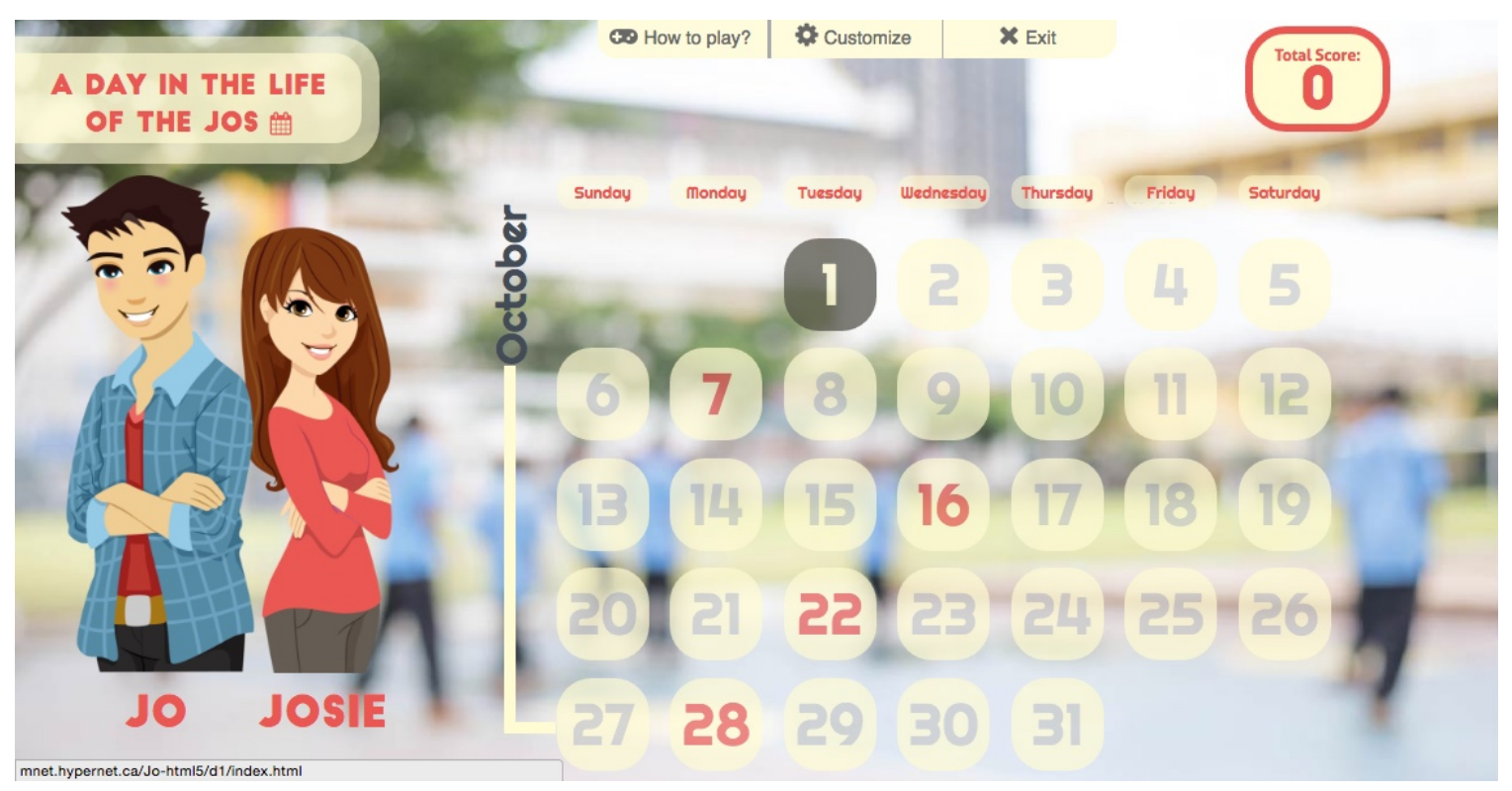

Figure 4.2: The main landing page of ADITL. A calendar shows the active days containing scenarios in red.

when Josie sees a post on her social media page saying that school busses are cancelled (Figure 4.4) and she must decide whether to trust this information. Options include checking with her friend who posted the picture and message, searching online to make sure that the information is correct, or checking the date of the post to ensure that it is recent. In this case, the second choice is the best decision. All three choices imply that it is best to verify information that you see online, but some methods are more trustworthy.

\subsubsection{Game mechanics}

We selected the game mechanics based on principles of game design [86] [35] [70] [22]. We focused on mechanics that would encourage players' engagement while learning about online privacy and security issues and meet our entertainment and conversationprovoking goals from the analysis phase.

The following game mechanics were incorporated into our design:

Consequences: A consequence is the outcome of a certain action that the player takes in a game, where different outcomes are assigned different values [14] [22]. The main objective of the game is for players to realize that there are consequences for 


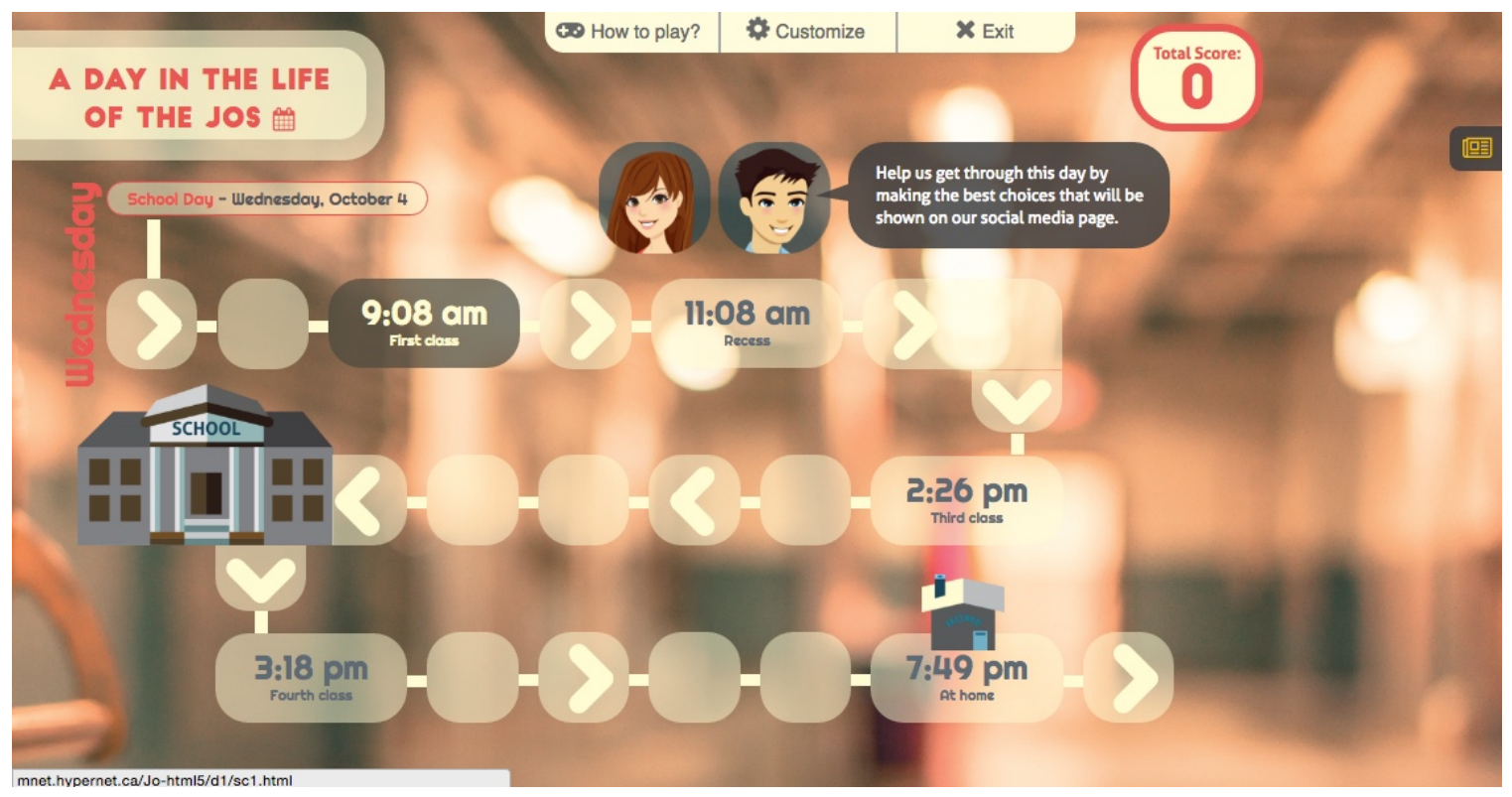

Figure 4.3: The Day View hosts links to scenarios for a particular topic.

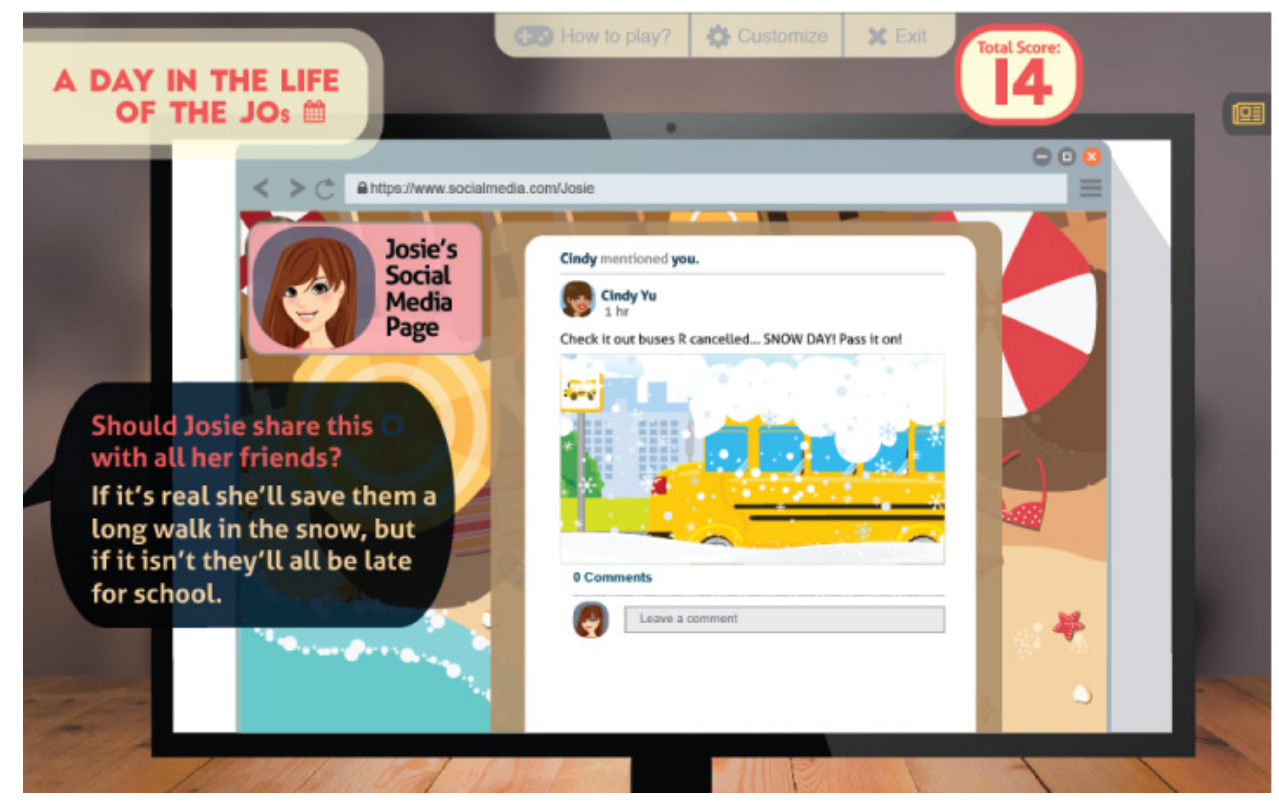

Figure 4.4: Scene from the online information verification scenario where Josie sees a post on her social media page with the news that school busses are cancelled and that it is a snow day. 
every decision they make. For example, a decision with a positive consequence can be that Jo or Josie gets a compliment or makes a new friend on their social media pages. While the children are going through ADITL, they can discuss the options to determine the ideal solution and consider the possible consequences of each choice, then reflect on the outcomes. ADITL scenarios are intended to reflect children's everyday life. This should also encourage children to share their personal experiences as they are going through the game. Each game choice results in visible consequences for the characters and we anticipate that these will be a discussion generator among the children. Often, appropriate choices are not immediately apparent and may have unexpected consequences. The game helps the player understand different consequences of their actions through feedback, as discussed next.

Feedback loop: Feedback is used to guide the players towards the correct outcome. Feedback is most effective when indicating that the chosen action was incorrect, without explicitly telling them the right action [35]. In ADITL, feedback accentuates the positive or negative consequences that occurs based on the player's choice. We add score keeping and a feedback loop [35] as two main features of the new game; research has shown that both were successful in encouraging players to continue playing a game [35]. In ADITL, score keeping is represented with a small score board located on the top right of the game screen, and appears on all the pages of the game. The score increases every time the player makes a right choice. Each choice is worth 0,1 , or 2 points, with players trying to gain the highest total score.

A newsfeed displays positive or negative consequences for Jo and Josie and allows the player to see the direct impact of their in-game choices on Jo and Josie's life. The newsfeed located on the top right side of the game's screen; it slides open once the player makes a choice and shows the repercussions of the player's choice as they unfold on social media. Both the score and the newsfeed are meant to engage the player and communicate the consequences of their in-game choices. They are meant to be used as educational tools in an indirect manner. Additionally, a pop-up message further explaining the consequences of the player's choice appears onscreen at the end of the scenario. Once the player is done reading, they can close it at their own pace. The pop-up message is meant to accentuate and reinforce the learning, in case the player 
did not fully notice the changes which happened on the Jos' newsfeed as a result of their choice.

We intentionally created nuanced situations to teach the children how to respond to situations where the answer is not clear. For example, in the first scenario of D1 cyberbullying (Figure 4.5 to Figure 4.7), Josie receives a text message that her friend Cindy has a crush on Brian. The screen then changes to show three choices: 1) Check with the sender of the message on its origins, 2) Share the news with her brother Jo, 3) Post a nice comment on Cindy's page. Each choice carries a positive or negative consequence and a certain score. If the player chooses the third choice (Figure 4.7), for example, then the player gets 0 points and the newsfeed updates to show Josie's post (Figure 4.8). A post appears from Cindy blaming Josie for leaking a secret. Then a third post appears, saying that Cindy unfriended Josie on her social media page.

As a more direct educational feature, each day ends with a summary page reiterating the player's choices and commenting on the appropriateness of each. The player gains perspective of the day by reviewing a summary of all their choices for that topic.

Achievement: Achievement in a game can be provided in the shape of badges, points, and/or reward points [35]. Achievement is important as it acts as the extrinsic motivator for the player to continue playing the game. By tracking their achievement throughout the game (by following the game score), players are motivated to continue playing once they got a score point. In ADITL, the "right" choice will gain them 2 points, the less optimal choices provide 1 point each, and the "wrong" choice earns 0 points. A higher the score indicates that the player is doing well.

Humour: Humour relaxes tension during learning [54]. It also acts as a trust builder, especially in learning situations [10]. Humour is especially important in game design, where it communicates to the player the message that "I am like you" and encourages the player to bond with the game characters. In ADITL, different scenarios carry humorous aspects to maintain a lighthearted mood throughout the learning. The characters find themselves in funny situations, illustrations depict humorous details, and the newsfeed posts include funny comments and images. The Jos 


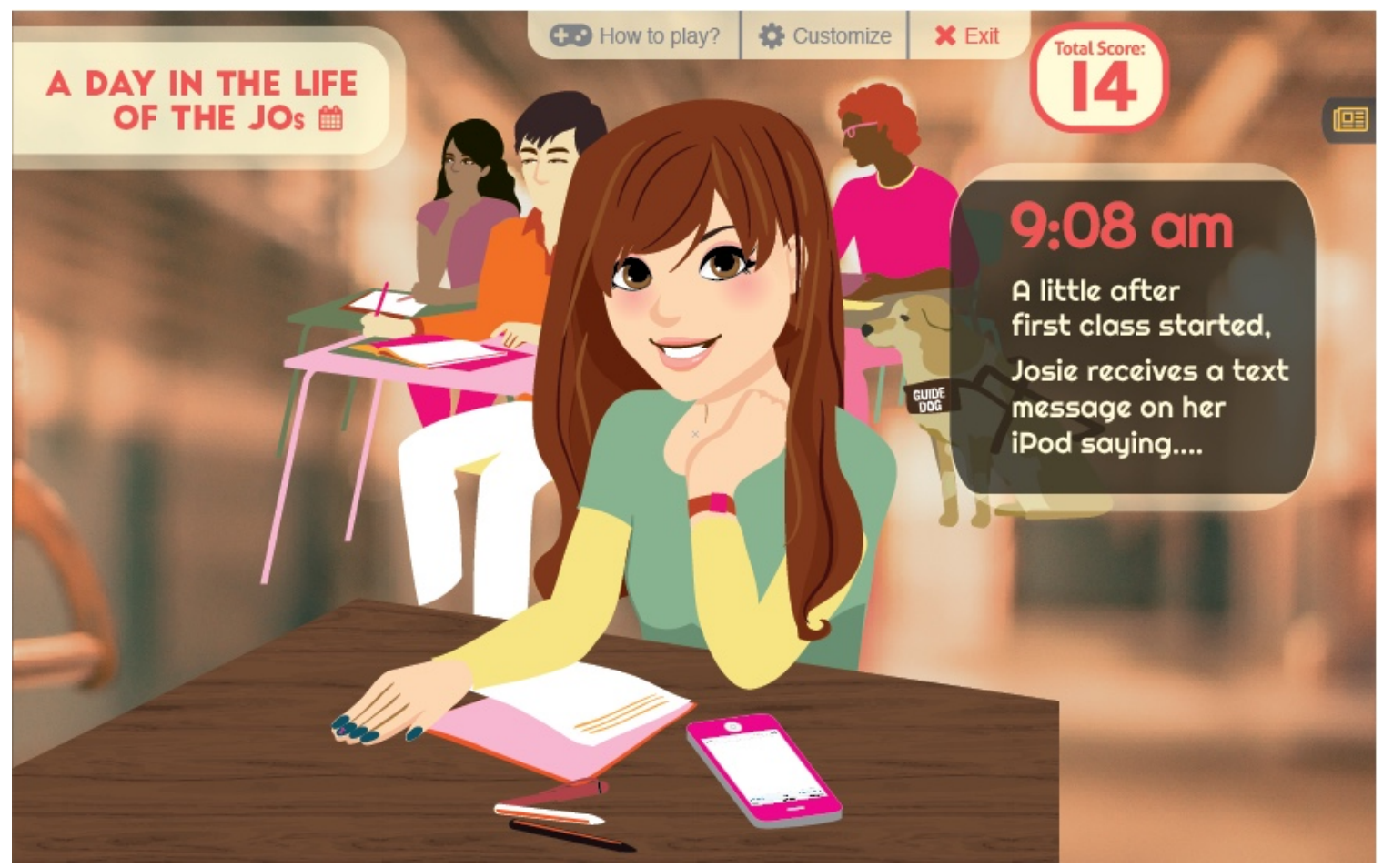

Figure 4.5: First scene of the first scenario in D1

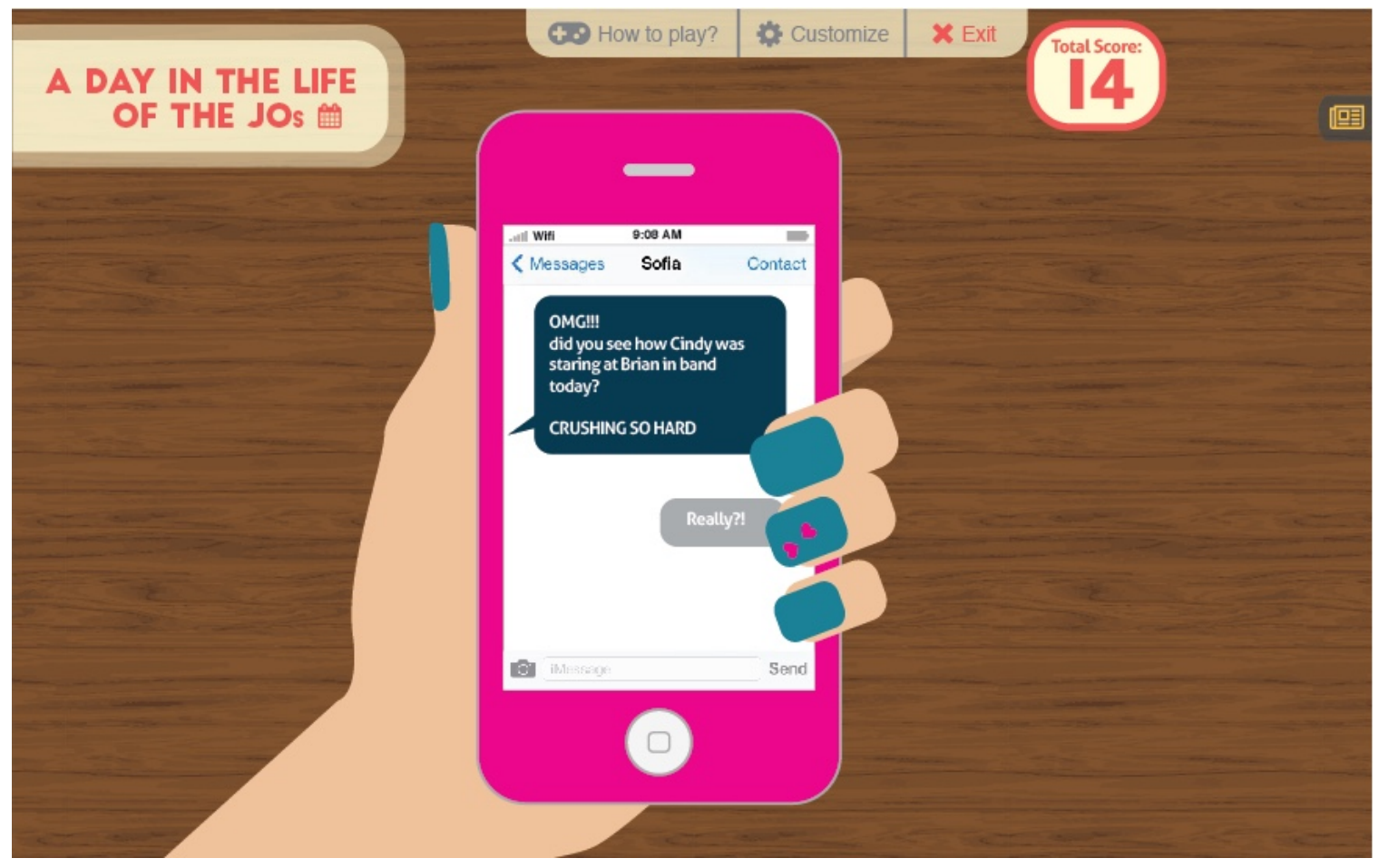

Figure 4.6: Second scene of the first scenario in D1 


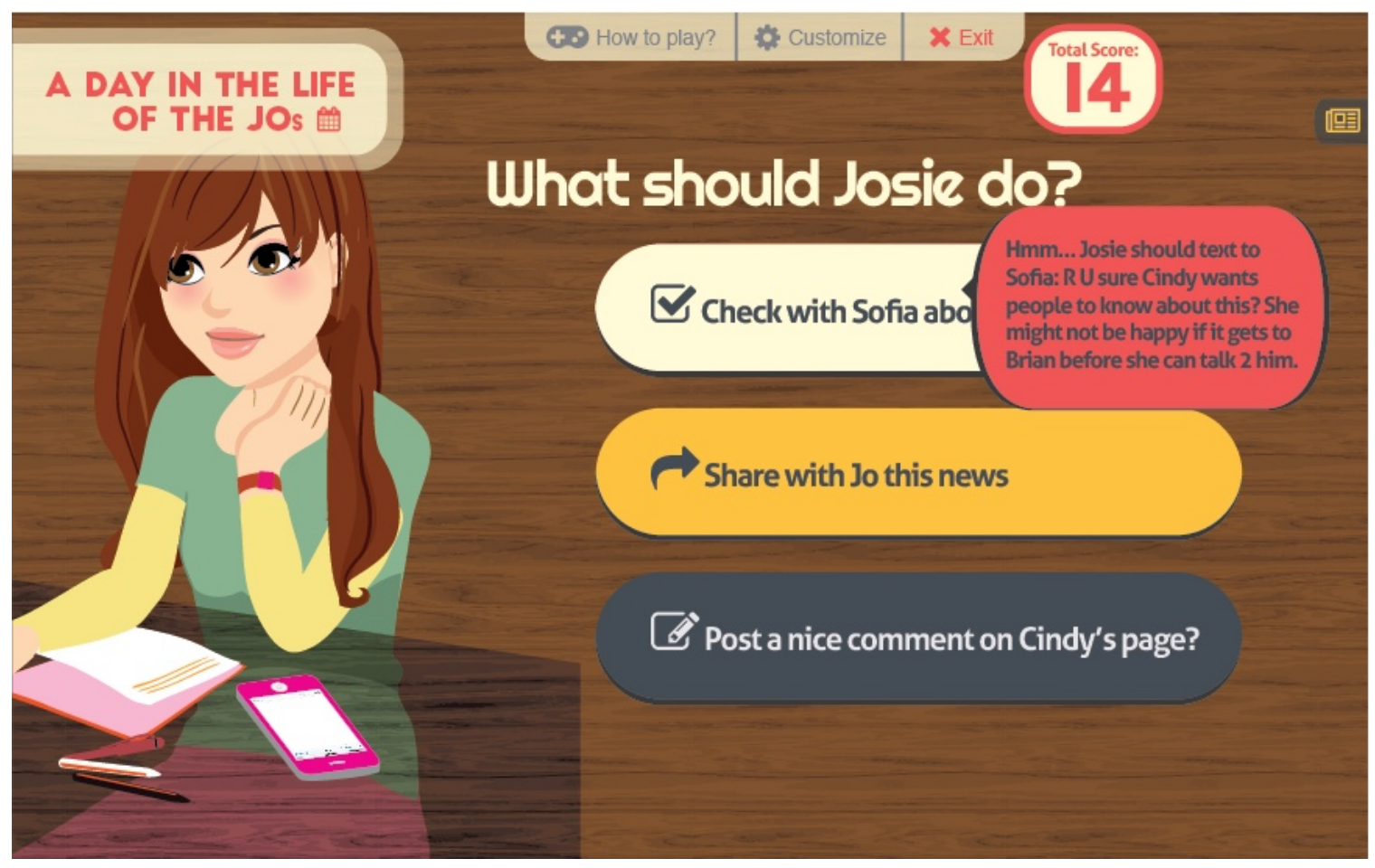

Figure 4.7: Third scene of the first scenario in D1

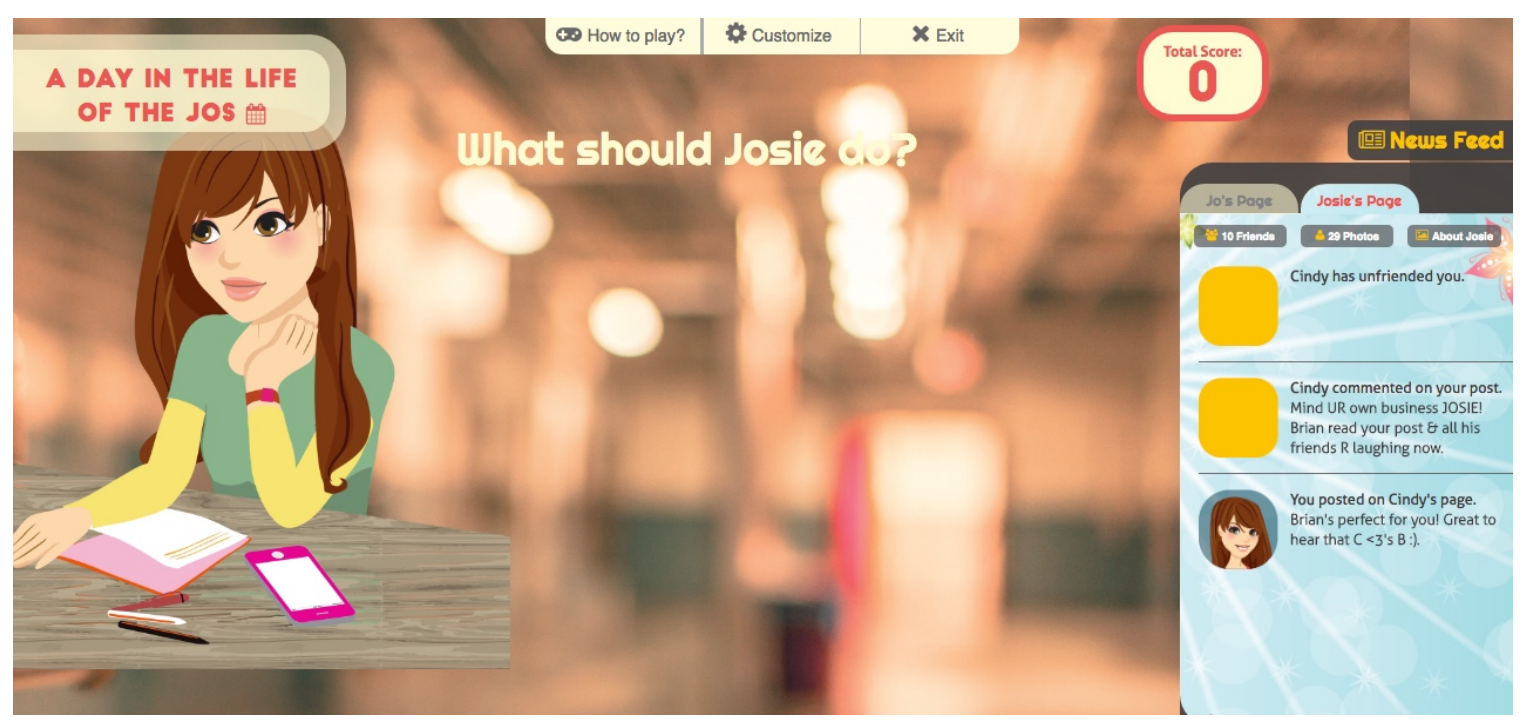

Figure 4.8: After the player makes a choice, the newsfeed opens to show posts on Josie's social media page. 


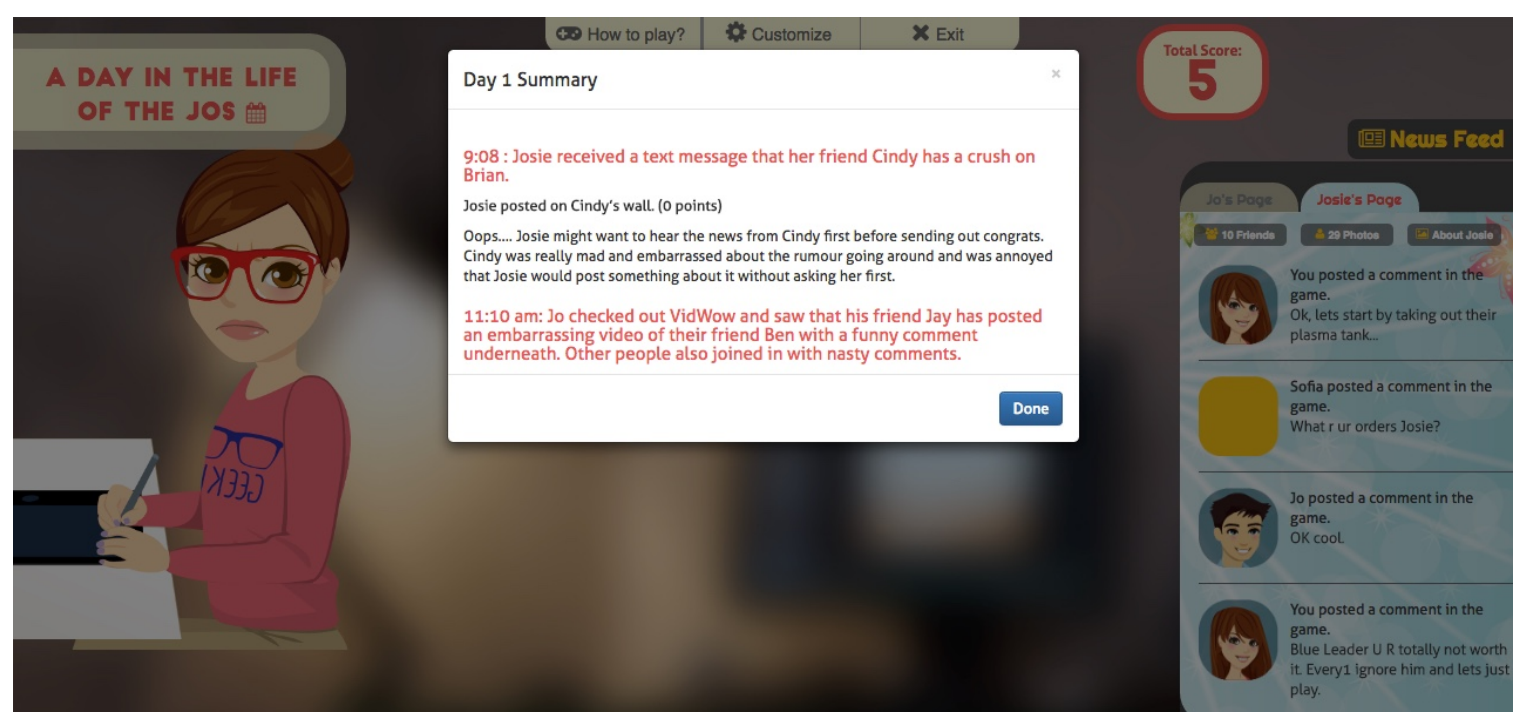

Figure 4.9: The end of day summary. The player can scroll to see all of their choices with scores and feedback.

face humorous situations and consequences in each of the game topics. For example, Figure 4.10 shows a funny photo of Sofia taken by Josie, and Figure 4.11 shows Josie with disheveled hair at a slumber party; both are mildly embarrassing situations that players may have experienced or could imagine themselves experiencing in real life. We made sure that the humour did not dilute the learned lesson behind each situation.

\subsection{Development phase: First ADITL prototype}

First ADITL Prototype: As a first prototype, we focused on two aspects where feedback from users would be most useful. We devised visual design alternatives and we developed the storyline for the first day of the game.

\subsubsection{Visual design}

We developed 3 different visual designs for ADITL. Based on participants' feedback of the old game, we kept the two main characters Jo and Josie, but updated their appearance. We designed three concepts with different look-and-feel for the characters, colours, and backgrounds. Figures 4.12 to 4.16 show the three concepts. We user tested the three designs to decide on the most appealing characters and visual style. 


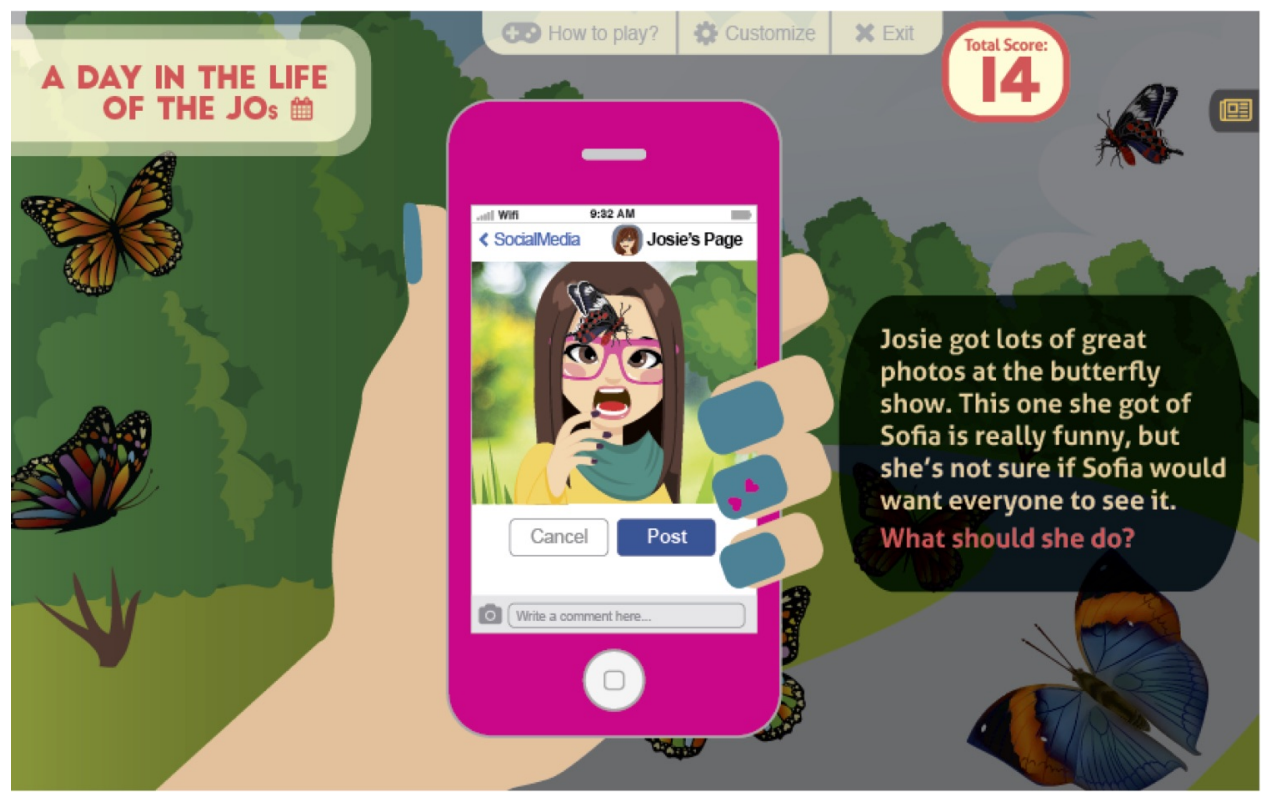

Figure 4.10: Scene from the photo sharing scenario showing Josie on a field trip and taking picture of her friend Sofia screaming because a butterfly landed on her face.

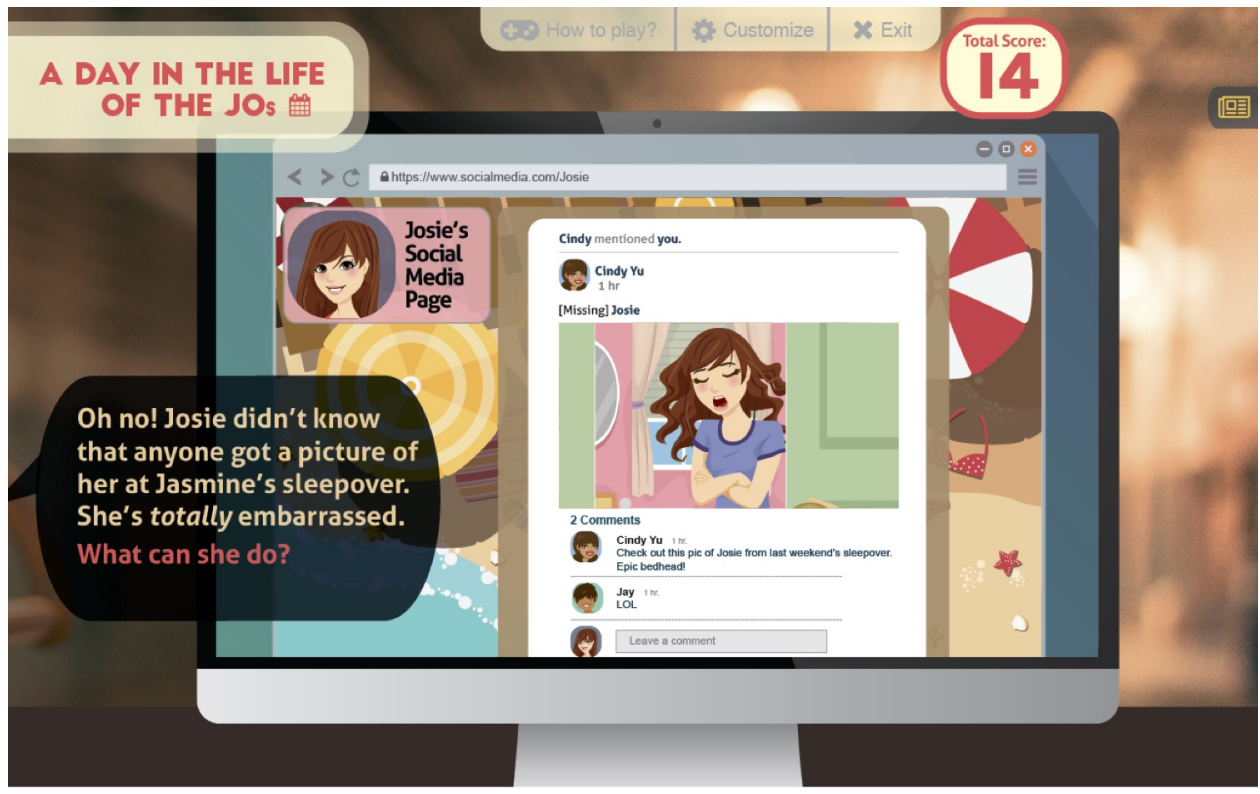

Figure 4.11: Scene from the online privacy scenario showing Josie looking disheveled at a slumber party. 


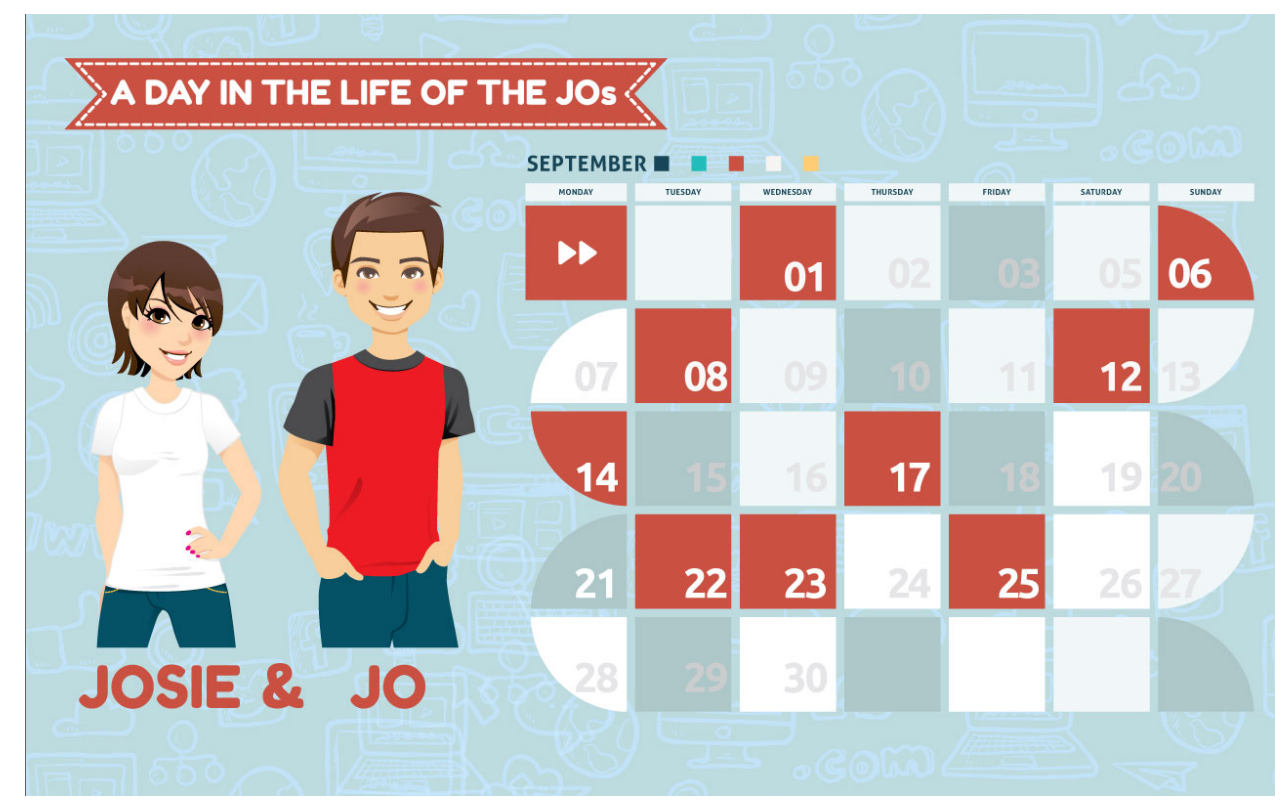

Figure 4.12: First Concept's calendar landing page.

Figures 4.12 and 4.13 show the First Concept, with younger-looking Jos. The colour scheme is blues and earthy reds. The landing page shows the calendar view with few active game days in red. Each game day represents one of the privacy and security topics (see Section 4.3.2 for a detailed description of the game topics). Once the player chooses a game day, they are presented with a day view (Figure 4.3).

Figure 4.14 shows the Second Concept with only the landing page, since the layout is similar to the First Concept. Difference include the colour scheme, the use of icons for the different days/topics, and the appearance of the Jos, who are shown full figure and appear older. Figure 4.15 and 4.16 show the Third Concept with a different look-and-feel. Here Jo and Josie also look older than in the First Concept. The background is a blurred photo, which adds more depth than the two-dimensional illustrated backgrounds of the First and Second Concepts. The calendar days on the landing page are bubbles rather than square. The day view follows the same style.

\subsubsection{D1 storyline}

For the first prototype, we developed the storyline for D1, where the Jos must deal with cyberbullying situations. The scenarios were composed as written stories and 


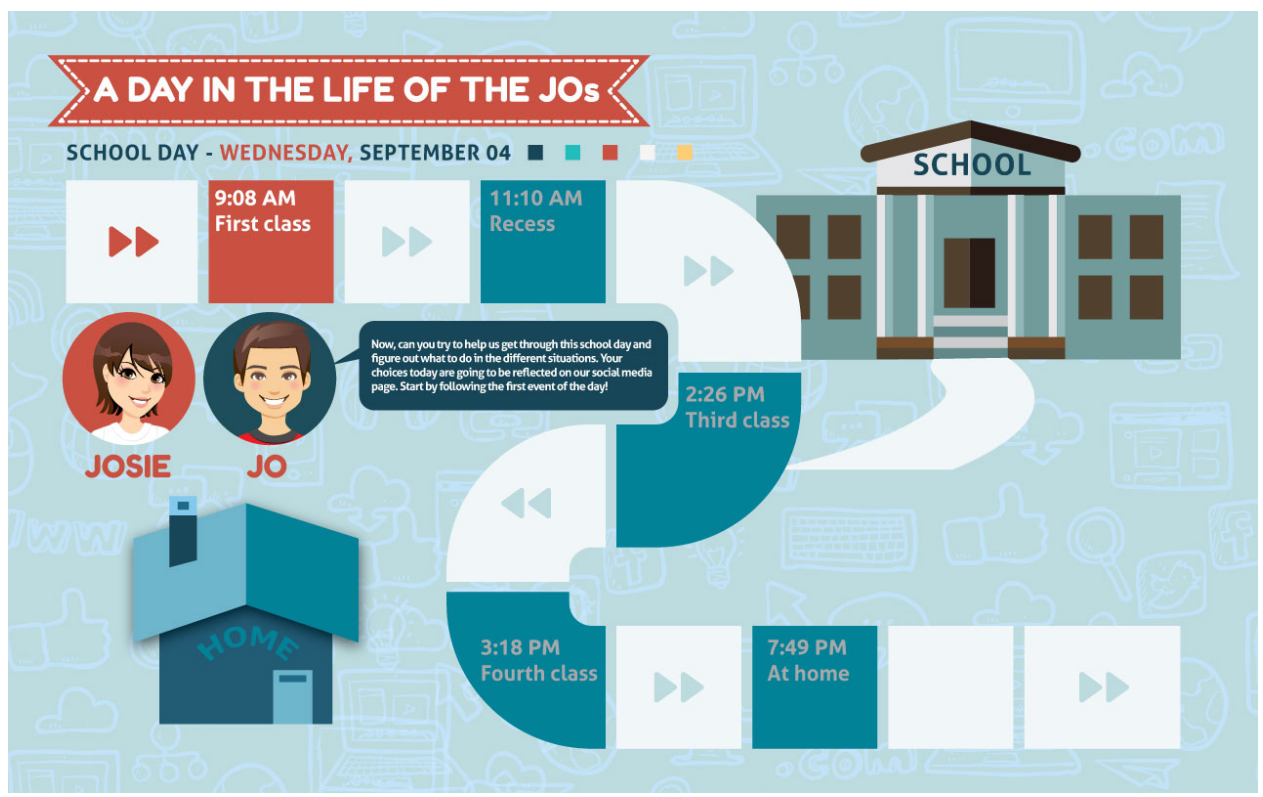

Figure 4.13: First Concept's day view.

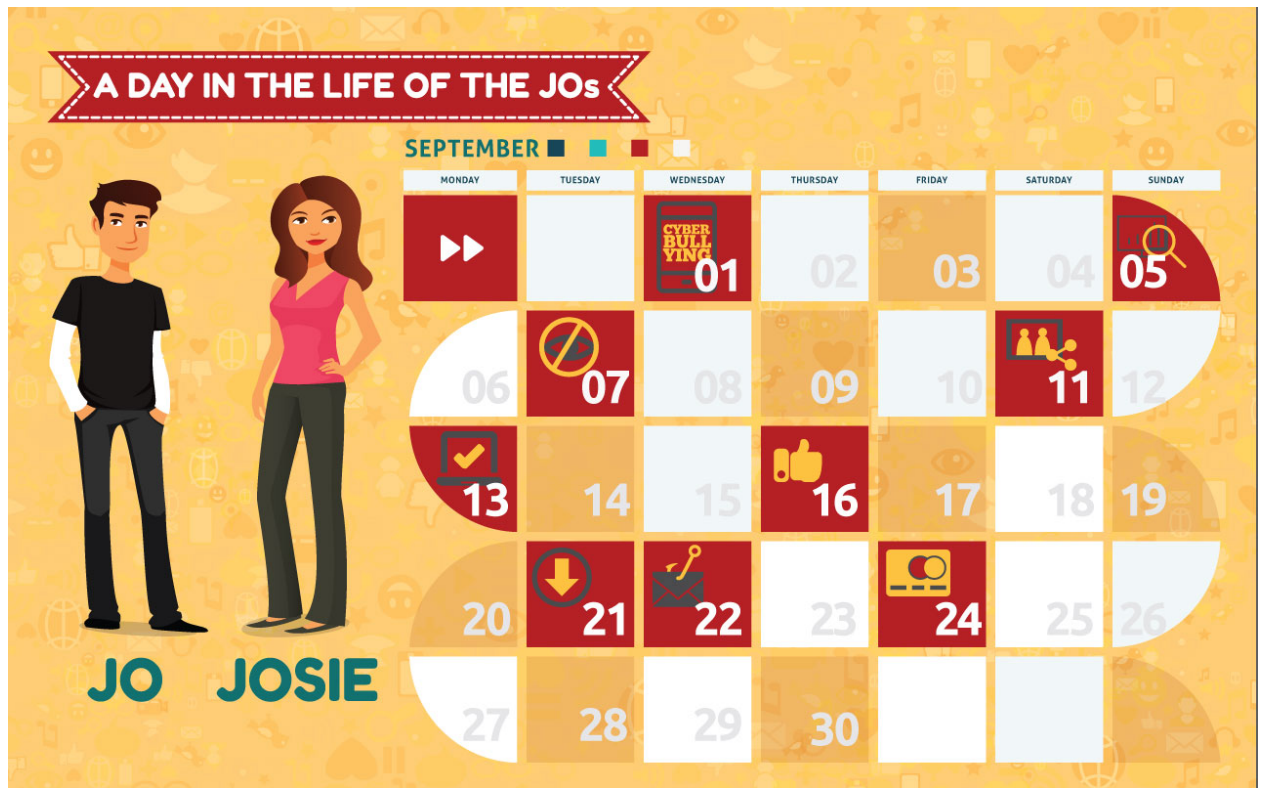

Figure 4.14: Second Concept: The landing page of the second concept, including icons on the active calendar days. 


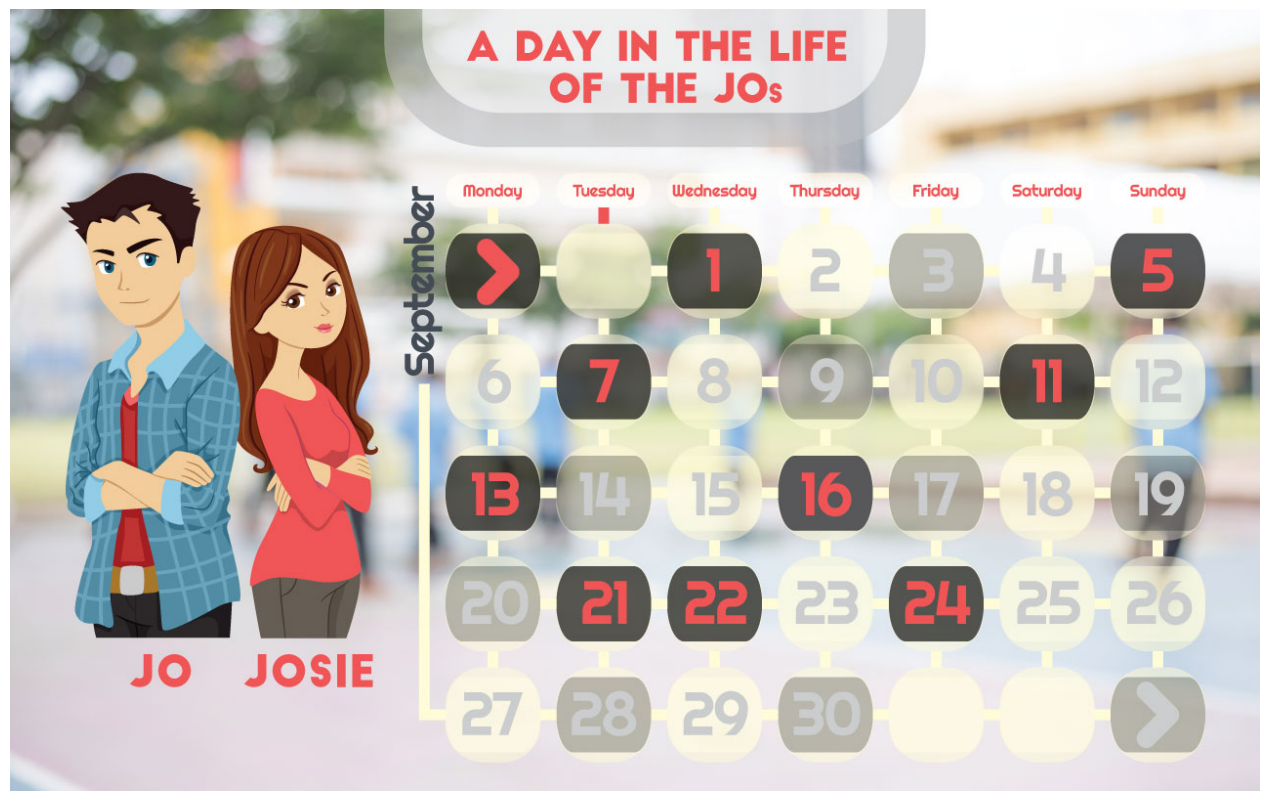

Figure 4.15: Third Concept's calendar landing page.

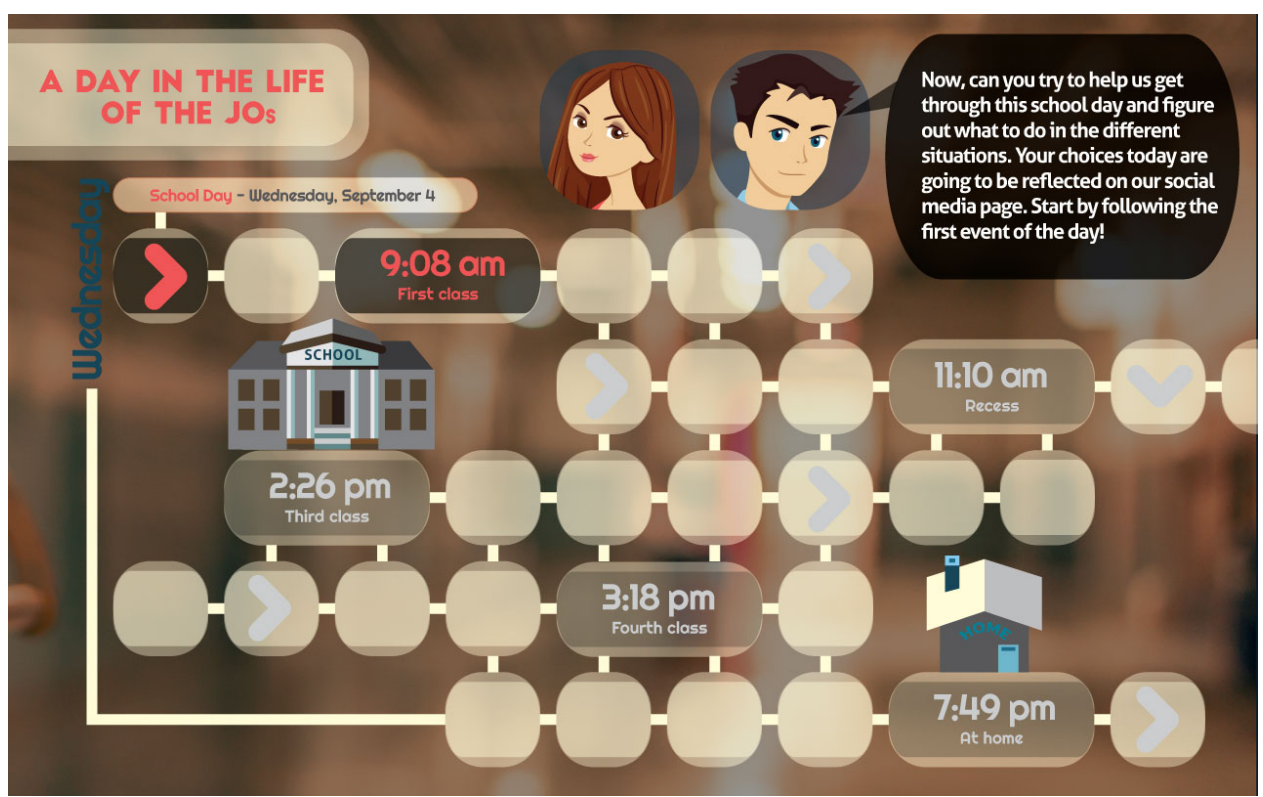

Figure 4.16: Third Concept's day view. 
ended with a question about what Jo or Josie should do in the given situation. The scenarios had no visual representation at this stage.

In the first scenario, Josie receives a text message saying that one of her friends has a crush on another boy in the class. Josie is faced with a decision to spread the rumour further by sharing it with her brother, checking with the original sender of the message on the validity of this news, or congratulating Cindy directly on her social media page. For scenario 2, Jo's friend shows him a video taken last week of another boy in class; the boy was sleeping and others drew all over his face with markers. The scenario asks if Jo should add to the funny comments written about the video, or should flag the video as inappropriate, or should post a comment supporting the abused friend. In the third scenario, Jo is working on school's yearbook and comes across an old photo of the principal with a funny hair style. Jo considers whether to publish the photo in the school's yearbook, or share the photo with his friends only, or to check with the principal if it is okay to publish the photo. The fourth scenario portrays Jo watching a conversation among his basketball teammates on their online group chat. Team members are attacking Matt and accusing him for letting them lose the last game. Jo's choices are to standup for Matt by writing a comment telling the team to stop harassing him, leave the group chat before the rest of his team members notice his presence, or send Matt a private message giving him moral support. The fifth scenario shows Josie at home playing an online game with her team members when she notices a verbal attack on her brother Jo from a player from another team.

Josie, as leader of her team, is faced with the following choices: reprimand the player for his behaviour and ask her team to mute him, stand up for Jo and attack that player in return, or tell her team to start trash-talking the player. Appendix A.7 offers a full script of cyberbullying day.

\subsection{Evaluation Phase 1: Pilot Study}

We conducted a pilot user study to test the likability of the three different concepts for the ADITL game. We tested with eight participants aged 10 to 12 years. We recruited four new participants and we asked four participants from the JCJF study to return so we could get their insight on what they thought in comparison with 

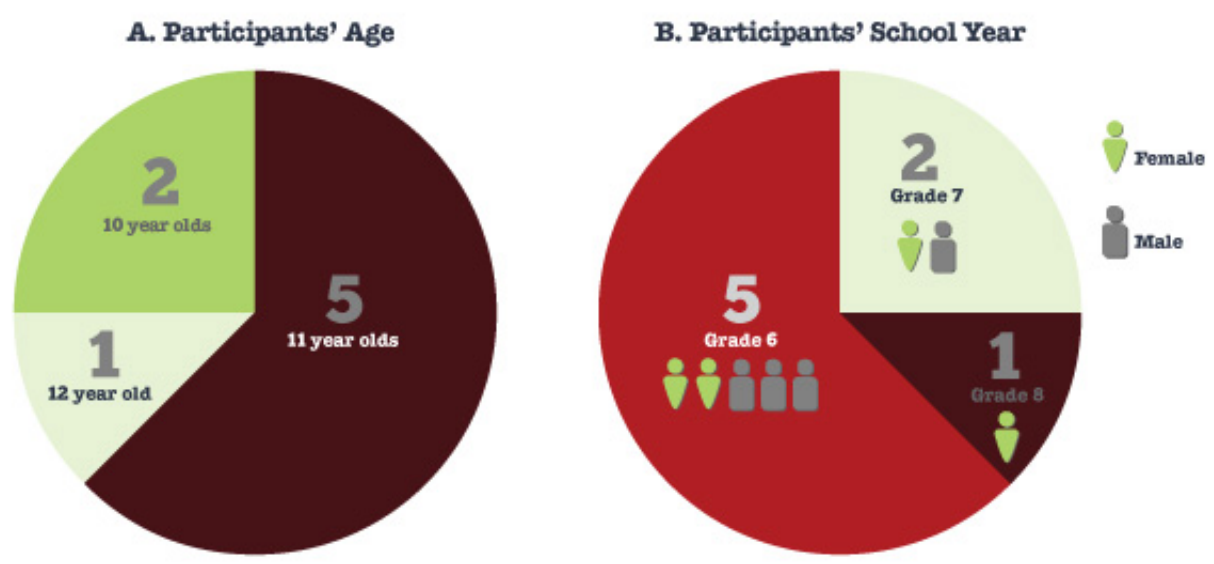

Figure 4.17: Demographics of the eight participants of the pilot user study.

JCJF. The participants were evenly split, four boys and four girls, with an average age of 11 years. Figure 4.17 illustrates the ages and school grades of the participants.

We recruited participants using the same method as in the previous user study, via Facebook parent and community group pages. A public announcement was posted and participants contacted the researcher via Facebook direct messaging. Returning participants were contacted by the researcher via the same method.

The study took place in local public libraries and community centres as convenient to the participants. This study is a continuation of the previous study, which was cleared by the Carleton University Research Ethics Board.

\subsubsection{Procedure}

The one-on-one sessions lasted 45 minutes on average and were recorded using a portable voice recorder. The sessions ran according to the following steps:

1. Consent form: Participants were greeted and parents completed the parental permission form (see Appendix A.2). Verbal informed assent (see Appendix A.3) was obtained from the child.

2. Concepts viewing: Participants were shown screenshots of the three concepts in a random order on the researcher's laptop and asked for their opinion. We asked 
questions about the children's preference for characters, layout, background, and colour to help them articulate their choices.

3. Scenario Reading: We tested the five scenarios from the cyberbullying day. We read out loud each scenario to the participant and asked for their decision about what the character should do. Table 4.1 summarizes the number of scenarios tested by each participant. In the new game structure, the scenarios are sequential and build on each other, therefore if we ran out of time in a session, the last scenario was dropped. We collected the participants' choices for the five scenarios verbally via a voice recorder.

4. Conclusion: Each participant received a $\$ 20$ dollar gift certificate as a compensation. The parent were given a debriefing form (see Appendix A.4) and was asked to sign a receipt of payment.

\subsubsection{Data analysis}

We coded and analyzed participants' choices for the three concepts, as well as for the cyberbullying day scenarios. Participants were assigned pseudo-names (P1 to P8). The four returning participants coded as P3R, P4R, P5R, and P6R. New participants were coded as P1N, P2N, P7N, and P8N.

The results of the pilot study are organized according to visual design and game design.

\subsubsection{Feedback on Visual Design}

Table 4.2 shows each participant's preferences among the three concepts for character, calendar, and background styles.

Game characters: Four participants preferred the character style of the Third Concept, three preferred those of the First Concept, and only one participant preferred the character style of the second concept. P2N, for example, particularly liked the facial expressions of the First Concept characters. P5R mentioned how she liked the Third Concept's older characters with their arms crossed. She mentioned that they look a lot like teenagers. She appreciated the fact that she will get to know what it is 
like to be older, and what kind of issues they might face, by going through the events of their day. P7N was also able to relate to the characters of the Third Concept, by saying: "They looked a little bit older than me, but not too old." P6R chose the Second Concept's characters with the full body profiles. However, after a second look, he mentioned that characters of the Third Concept resembled Anime characters and that kids his age enjoy Anime.

\begin{tabular}{|l|r|}
\hline Participants & No. of Scenarios \\
\hline P1N & 4 \\
P2N & 4 \\
P3R & 5 \\
P4R & 4 \\
P5R & 4 \\
P6R & 5 \\
P7N & 5 \\
P8N & 5 \\
\hline \hline
\end{tabular}

Table 4.1: The number of scenarios tested per participant.

\begin{tabular}{|c|c|c|c|}
\hline Participants & Character Style & Calendar Style & Background Style \\
\hline P1N & 3rd & 1st & 3rd \\
P2N & 1 st & 3rd & 3rd \\
P3R & 1 st & 1 st & 1 st \\
P4R & 1 st & 3rd & 3rd \\
P5R & 3rd & 3rd & 3rd \\
P6R & 3rd & 3rd & 3rd \\
P7N & 3rd & 3rd & 3rd \\
P8N & 2nd & 2nd & 2nd \\
\hline
\end{tabular}

Table 4.2: Participants' preferred character, calendar, and background styles from among the three concepts.

Calendar layout: Five participants preferred the look-and-feel of the Third Concept, while two preferred the First Concept's calendar, and only one participant chose the Second Concept's calendar view. P2N liked the calendar style of the Third Concept. She thought the days and numbers were large and clear. P4R liked the bubble shapes of the calendar days and the day view on the Third Concept. 
Background and colour scheme: Six participants were in favour of the background and colour scheme of the Third Concept. The First and Second Concepts received only one vote each. P5R stressed how she liked the blurred background and the colours of the Third Concept, describing them as "modern looking". P1N, P4R, P6R, and P7N reported similar opinions.

Day view: Six participants were confused by the Third Concept's day view layout. P4R found that there are too many bubbles on the day view and found them confusing to follow. She would prefer combining the First Concept's day view layout and the Third Concept style and background. Four participants preferred the look of the Third Concept day view but wanted to replace the layout with that of the First Concept.

\subsubsection{Feedback on Game Design}

Overall opinion of the game and scenarios: All participants liked the game and would like to play it in the future. P5R thought that cartoons are a great way to engage people, because they are fun to watch and read. P4R also mentioned that she prefers comics to real people's photos. She explained that a live TV show holds people's attention less than one with comics. After going through the scenarios verbally, P1N suggested that the game should include more visuals, where the picture would describe the situation, instead of reading through the scenarios.

Most participants had a strong sense of what is wrong and what is right. For example, their answers in some scenarios advocated confronting the bully and asking them to stop, or reporting the situation to an adult. However, the situation of the bullied person can become worse if the bully finds out that someone "snitched". Svahn explains that bullying "is an act of resistance to adults' involvement in children's peer interactions" [81]. In the first scenario, Josie received a text message saying that Cindy had a crush on Brian. One participant mentioned that Josie should go directly and confront Cindy. If Cindy tries to avoid answering then this means that she's trying to hide something and that the rumour is true. Such attitude shows poor empathy towards Cindy who is the focus of an on-going rumour, even if it harboured traces of truth. This suggests including scenarios where the line between right and 
wrong can be murky, in order to teach the children about more nuanced situations.

\subsection{Implementation Phase: Second ADITL Prototype}

Design choices: We made the following design decisions for the next prototype of the game based on results from the pilot study. In the pilot study, participants preferred the character style of the Third and the First Concepts. We combined the facial expressions from the First Concept and the character bodies of the Third Concept. Figure 4.1 shows the new characters.

$80 \%$ of participants preferred the style and colour scheme of the Third Concept. However, they thought that the day view layout was confusing and preferred the layout from the First Concept. We simplified the Third Concept's layout as presented in Figure 4.2.

Based on the feedback, we designed ADITL to make extensive use of visuals. One participant suggested having visuals explain the scenes instead of reading through the scenarios. We minimized text, while maintaining context for the lessons. Each scenario is illustrated with colourful visuals to draw the children's attention to the intricacies of the scene.

The game was designed and prototyped in Adobe Illustrator CS6. The illustrations were created using customized stock art. We combined and modified the work of three different stock artists [27] [5] [52] to create the characters and scenery of the game. The researcher has a background in graphic design and designed the look and feel of the game elements, the game interactions, the layout style, and the colour scheme. See Appendix A.8 for the 25 scenes.

Development: A second graduate student, Sana Maqsood, developed ADITL in HTML 5 and CSS3 using Adobe Dreamweaver CC. Most components have text and images decoupled to provide flexibility in layout. SVG images and their behaviours are defined in XML text files. They are characterized as being lightweight files that take minimal loading time, since all the vector images have been transformed to code. Game content and user tracking is stored in a mySQL database. It is formatted to accommodate laptops, desktops, and tablets.

Deployment: ADITL will be hosted on MediaSmart's website, and accessed by 
grades 6 and 7 classrooms nationwide.

Game Reporting: The game is intended for use within a classroom setting, so children's progress will be reported to the teacher. Using a custom Shell program hosted by MediaSmarts, the teacher will be able to create a unique identifier for each child. The children will use their ID to log in to the game. The Shell records the timestamps and details of the child's performance, making both available to the teacher.

The final step of the ADDIE process, a user study evaluating ADITL, is reported in Chapter 5. Chapter 6 will discuss the implications of our work, its limitations, and our conclusion. 


\section{Chapter 5}

\section{Evaluation of the new game: "A Day in The Life of the Jos"}

As part of the ADDIE evaluation phase, we conducted a user study to assess the overall effectiveness of the new game design, "A Day In The Life of The Jos" (ADITL). Our goal was assess the effectiveness and likability of the design, as well as the content of the new game.

\subsection{Methodology}

Following a similar procedure as with the JCJF user study from Chapter 3, we tested ADITL [43] using an eye tracker. The user study included semi-structured interviews to gauge the participants' knowledge of the online privacy threats. In this section, we discuss the participants' demographics, recruitment process, and interview procedures.

\subsubsection{Demographics}

We recruited fourteen participants, eight girls and six boys to test ADITL. The participants were 11 to 13 years old (grades 6 to 8); Figure 5.1 shows the age distribution. For continuity, four participants were returning participants who had previously tested JCJF and the early ADITL prototypes. To identify them, an R will be appended to their username (e.g., P6R). Usernames for new participants will have an $\mathrm{N}$ appended (e.g., $\mathrm{P} 2 \mathrm{~N})$.

\subsubsection{Recruitment and Sampling}

The study was approved by the Research Ethics Board (REB) at Carleton University. We recruited participants locally through contacts at The Leading Note Foundation [61] and the affiliated kids program, Orkidstra [61]. The program's main focus is 

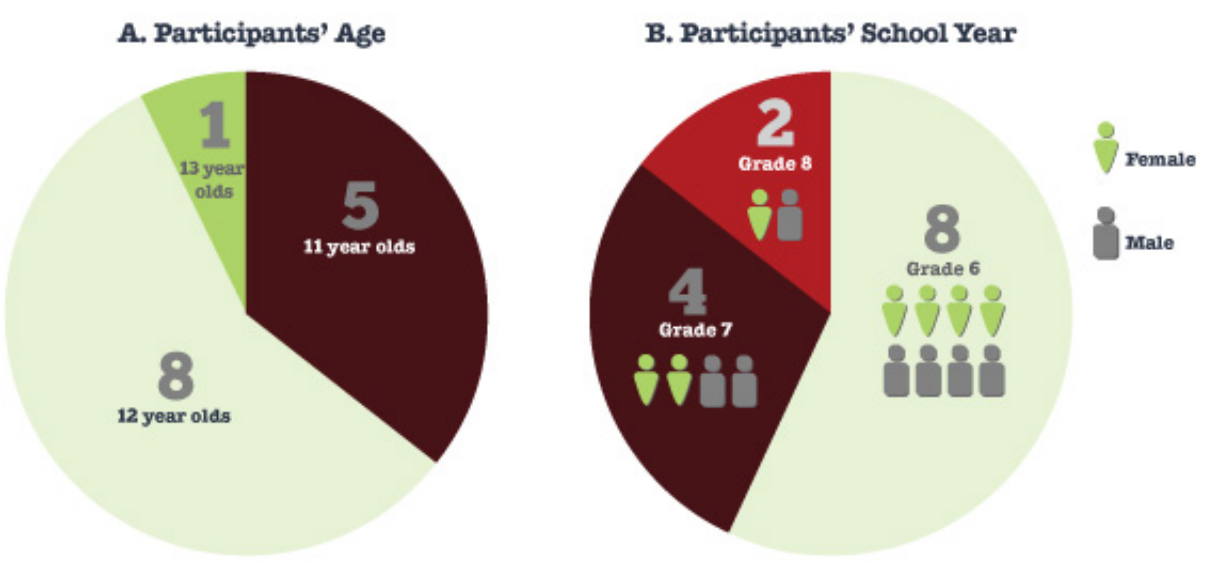

Figure 5.1: Demographics of the 14 participants of the ADITL user study.

to teach music to children of different age groups from underprivileged communities. A flyer announcing the user study and consent form was handed to students during a program session to bring home to their parents. If parents agreed for the children to participate in the study, they signed the form and students returned it at their next practice session at the foundation. The remaining four participants from the previous user studies were contacted via Facebook and in person.

\subsubsection{Procedure}

The study session lasted between 30 to 45 minutes and was conducted according to the following steps:

1. Consent form: Participants were greeted and the signed parental permission forms were gathered. We proceeded with the verbal informed assent and obtained the child's approval to move forward with the study.

2. Eye tracker: The SMI RED 250 MOBILE eye tracker with one-point calibration was used, along with the SMI Smart Recorder software to record the participants' on-screen eye gaze and the audio conversations. A webcam with its lens covered recorded the synched audio.

3. Pre-game-day interview: We asked participants questions in a semi-structured interview investigating basic understanding of the specific online privacy and 
security topic being tested. The same set of questions were repeated after viewing the related game content to measure if participants had gained any new knowledge of the topics.

4. Game day viewing: There were five game days in total (D1 - D5): D1) cyberbullying, D2) data mining, D3) online privacy, D4) photo-sharing of others, and D5) information verification. D1 was fully developed online with interactive features [43]. The other four days were presented as PowerPoint slides and the text relating to the outcomes for the participants' choices was shown on a printed sheet of paper. All 14 participants played Day 1 - cyberbullying (D1). Participants were then randomly assigned to play another game day (D2 - D5). Participants read through the scenarios and were encouraged to think out loud. They played the whole day before moving to the post-game-day questions. This happened with each of the game days they viewed.

5. Post-game-day interview: The pre-game-day questions were repeated. We also gathered qualitative data about the game in general. We asked about what the participants valued most and least in the scenarios they just viewed. Additionally, we investigated whether they would recommend ADITL to a friend or if they would like to play it in a classroom setting.

6. User feedback questionnaire: At the end of the session, participants completed a 5-point Likert scale questionnaire evaluating the game itself (see Appendix A.5). The questions asked about the content length, the scenarios, the amount of knowledge gained, and the game design.

7. Conclusion: We thanked the participants and offered a $\$ 20$ gift certificate as a compensation. Debriefing forms were then given to the participants to take home to their parents.

\subsection{Results}

Each participant played D1 and one additional game day. Table 5.1 summarizes the total number of participants for each day. P1N's eye tracking data for D1 was lost 
due to a technical error. P2N's verbal responses were lost due to an error with the audio recording for D1 and D3.

\begin{tabular}{|l|r|}
\hline Scenarios & No. of Participants \\
\hline D1 & 14 \\
D2 & 4 \\
D3 & 4 \\
D4 & 3 \\
D5 & 3 \\
\hline \hline
\end{tabular}

Table 5.1: Total number of participants who viewed each scenario.

\subsubsection{Pre- and Post-game-day Interviews}

The pre- and post-game-day verbal questions focused on gauging the participants' understanding of the main topics for each day. Two content-related questions were repeated before and after playing each game day. Appendix A.6 has the full question set. We manually coded participants' answers with 3 for an excellent answer, 2 for a marginal answer, and 1 for a poor answer. Figure 5.2 and Figure 5.3 show scores for the two questions from five game days. Excellent answers showed good understanding of the cyberbullying concept. For example, P6R stated that cyberbullying is bullying on the Internet and that people think they can get away with it, because of the anonymity factor. She elaborated by saying: "Sometimes they do it on Facebook. They put a mean comment and no one can really do anything about it except parents." Such an answer showed that the participant has an advanced understanding of the topic. Marginal answers had partial or superficial understanding. For example, regarding what she would do if she saw a person bullied online, P4N mentioned she would tell the bully to stop. After playing the game, she expanded her answer to also give support to the person being bullied, which is a concept mentioned in the game, and increasing her post-test score to excellent. A poor answer was assigned if the participant was not able to answer the questions in any form. For example, when asked what to do if she saw someone being bullied online, P1N answered that she was not sure. 


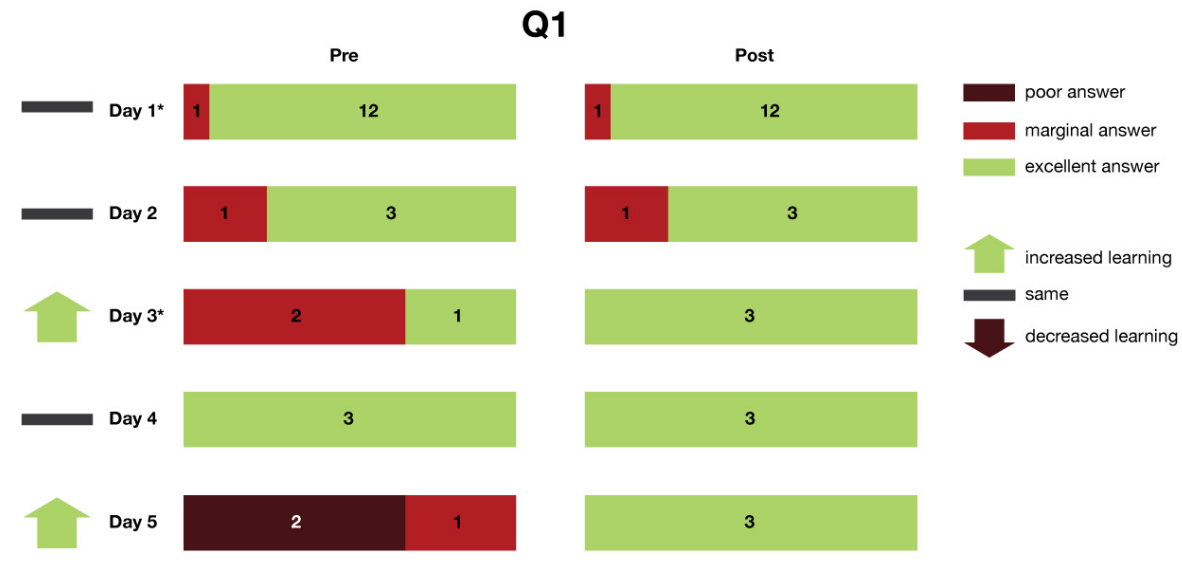

Figure 5.2: Distribution of scores for each for question 1 from the pre- and post-game day interviews. D1* and D3* are missing the recording of P2N.

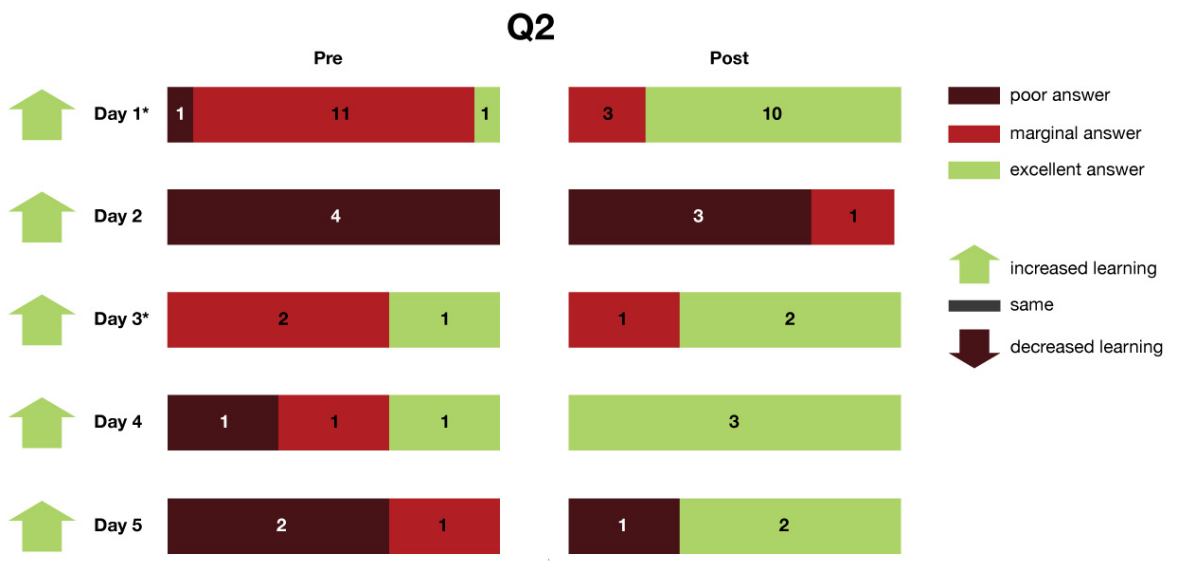

Figure 5.3: Distribution of scores for each for question 2 from the pre- and post-game day interviews. D1* and D3* are missing the recording of P2N. 
D1 - cyberbullying: The first question asked participants to define cyberbullying. 12 of the 13 recorded participants received the full score of 3 for their answers in the pre- and post-interviews, and one participant received a score of 2 in the pre- and post-interviews. Generally speaking, participants of this age group are familiar with the concept of cyberbullying. All agreed that it is bullying online, although some participants gave more examples of cyberbullying than others. In the post test, participants incorporated examples from the game scenarios, like "spreading a rumour," (P5N) and harassing someone online via social media and online games (P9N), which they did not mention before playing the game.

Question 2 asked how participants can help if they saw someone bullied online. Answers demonstrate considerable improvement in participants' understanding in the post-test. We noticed that participants were able to better articulate the type of help they could offer and gave diverse ways to help the bullied person if they witnessed an online bullying situation. Given the timing, we assume that this improvement in the participants' answers is due to the advice offered by the game scenarios. For example, P1N wasn't sure what to do in the pre-game day question, but she mentioned reporting the conversation or video to the social media site post-test. P6R added that besides reporting the bully, she could give support to the bullied person, by reaching out and checking on how they were doing. P2N mentioned that the game offers a cool way to learn new things about cyberbullying, and that she liked how it explains how to make the right choices.

D2 - data mining: Three of the four participants scored 3 for an excellent answer for the first question pre and post-test. We asked about the information that would be safe to share with websites and participants agreed that name, age, birthday, home or school addresses, and password are information that should be kept private. Most participants were skeptical of sharing credit card information with a website and mentioned that they avoid giving out any personal information "unless they really had to". This might be due to participants being exposed to this advice either through school or home conversations.

The second question discussed the definition of a website cookie. Participants 
were not familiar with website cookies or their functionality. Our goal was to understand participants' perception of the different ways websites collect personal data, however, during the game there were no mention of website cookies or their purpose. In hindsight, we should have asked a different question. However, it does serve a control question, showing what happens when the info is not provided in the game; participants remained unfamiliar with the concept post-game.

D3 - privacy: We tested with four participants, however, one participant's interview data was lost and was thus omitted for the pre and post answers. The first question checks on the meaning of online privacy. The post-game day viewing results show improvements in the participants' understanding of online privacy with all three participants receiving scores of 3 . They were able to provide more details describing components of their online privacy. For example, P14R described online privacy in the pre-test as: "keeping stuff that you don't want anyone else to know to yourself." In the post-test he gave more detailed description: "keeping stuff to yourself that you don't want anyone to see, like embarrassing pictures of you, stuff that you said to other people that wasn't good."

The second question asked participants to describe ways to protect their online privacy. All participants achieved the highest score in the post-test, compared to only one participant during the pre-test. As with the first question, participants' answers became more articulate and included explanations of ways to protect their online privacy after interacting with the game. For example, P10N initially mentioned using an antivirus as the main way to protect your online privacy against hacking. Then post-test, he added details like not "putting any stuff that you don't want (to be hacked, or) that people would want..., to take, or to show."

D4 - photo sharing: In response to the first question, all three participants showed strong agreement that they needed permission before sharing images of their friends online or offline The pre- and post-test results all scored 3 points.

The second question discussed the issue of image copyright and how to decide if a picture online can be used. Only one participant received a full score on the pre-test. P3N mentioned that if the picture was "appropriate" then she will use it. In the post-test, all participants received full scores. For example, P3N mentioned that you 
need to check for the license, and that she would check if it is okay to use the image. Additionally, she added that she would check with the person who took the picture.

D5 - verification of information from online sources: The first question asked how to verify online information sources. Two participants mentioned that you can't really tell whether information you find online is true. The third participant was not sure how to distinguish between fake and true information online. None of the participants received a full score for their answers prior to playing. After the game day viewing, all participants were able to identify several ways to verify online information. For example, P12N mentioned that you can check who posted the information and compare its information to other online sources, which are some of the tips found in the game scenarios.

The second question described a scenario and asked what to do if the participant saw an online post by a friend on a social media site. The post advertised a $\$ 50$ gift card giveaway by their favourite store. The participant was asked what they should do. No participants received the highest score for their pre game answers. However, two of three participants received a full score after playing. Pre game answers included checking with the person who posted the information and going to the store directly to check. Post game answers included verifying facts about the page or the post, like when it was posted, and cross referencing the information with other online sources.

\subsubsection{Game Choices}

Within the game, participants chose a course of action for the characters. Each choice was valued at 0,1 , or 2 points depending on the appropriateness of the action. We then calculated the mean score for each game scenario tested. Due to technical difficulties, P1N's D1 choices were not recorded.

Game scenarios' choices scored 0 for poor answer, 1 for marginal answer, and 2 for the best answer. Due to content decisions by MediaSmarts, Scenario 3 in D1 had one option valued at 1, and the other two game choices valued at 0 , and Scenario 5 had one option valued at 2, and the other two game choices valued at 0. D4 had one scenario with the full score as 2 , and the two other choices as 0 . This was an intentional pedagogical decision highlighting that sometimes decisions fall into a grey 


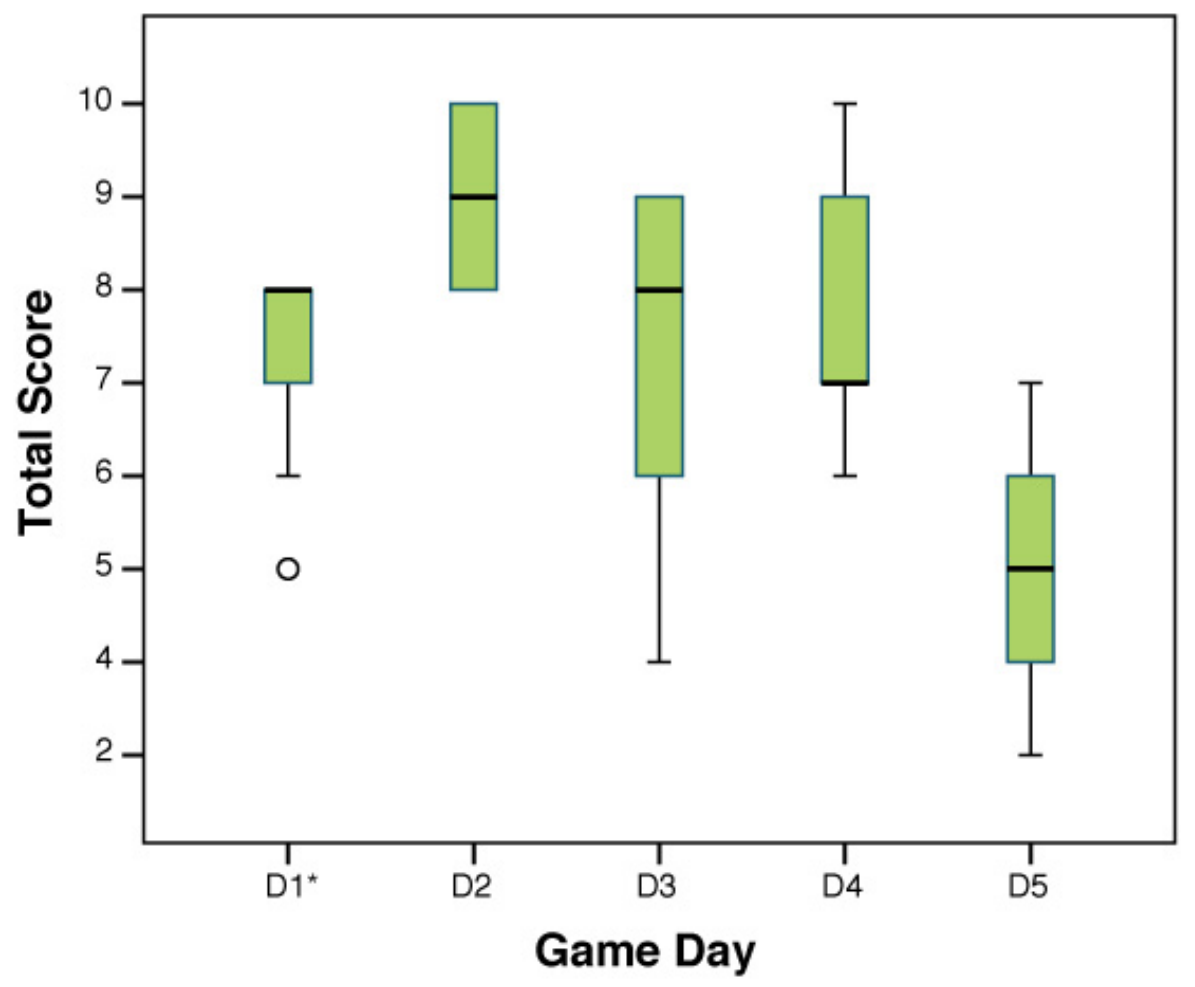

Figure 5.4: Total score earned per day for in-game decisions out of 10. *D1's maximum score is 9 .

area where it is unclear which is the "best" answer, and allowing the participants to choose the best option between two tricky situations.

Since each day had 5 scenario, each day could result in a maximum of 10 points, with exceptions noted above. Figure 5.4 shows the total scores earned by participants for each day. Additionally, we calculated the total score earned for each scenario of the five game days, shown in Figure 5.5. Participants received the lowest score with D5. D5's topic, verification of information from online sources, is not widely discussed among this age group or with the adults in their lives. Overall, differences in knowledge were not age-dependent. Some younger participants showed more knowledge about advanced topics than older participants. 

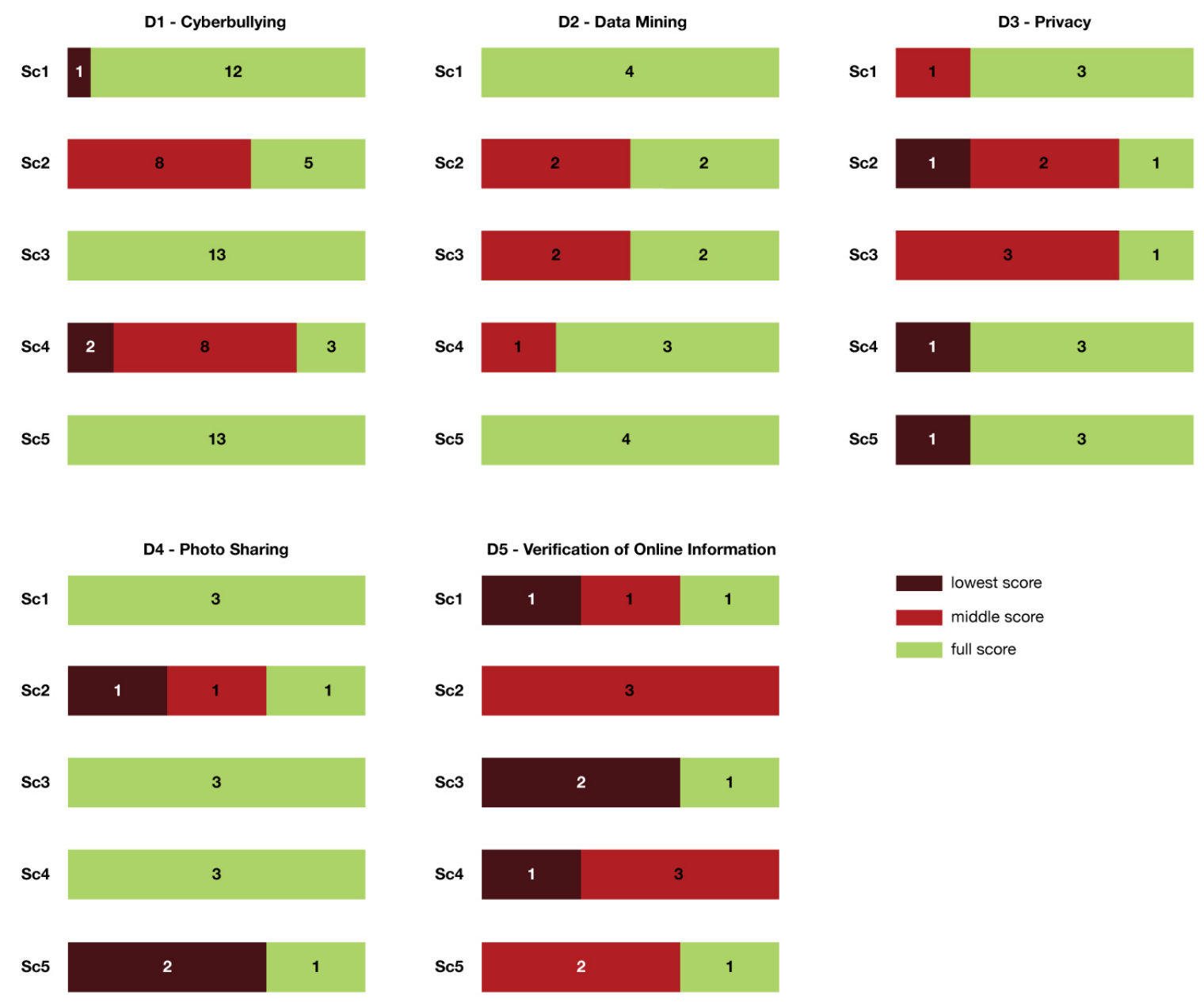

Figure 5.5: Number of participants earning each game score for the 5 scenarios of the 5 game days. Three scenarios differed from the norm. D1-Sc3 had a total score of 1 for one choice, and the other two choices were valued at 0. D1-Sc5 and D4-Sc5 had a total score of 2 for one choice, with the other two choices valued at 0 . 


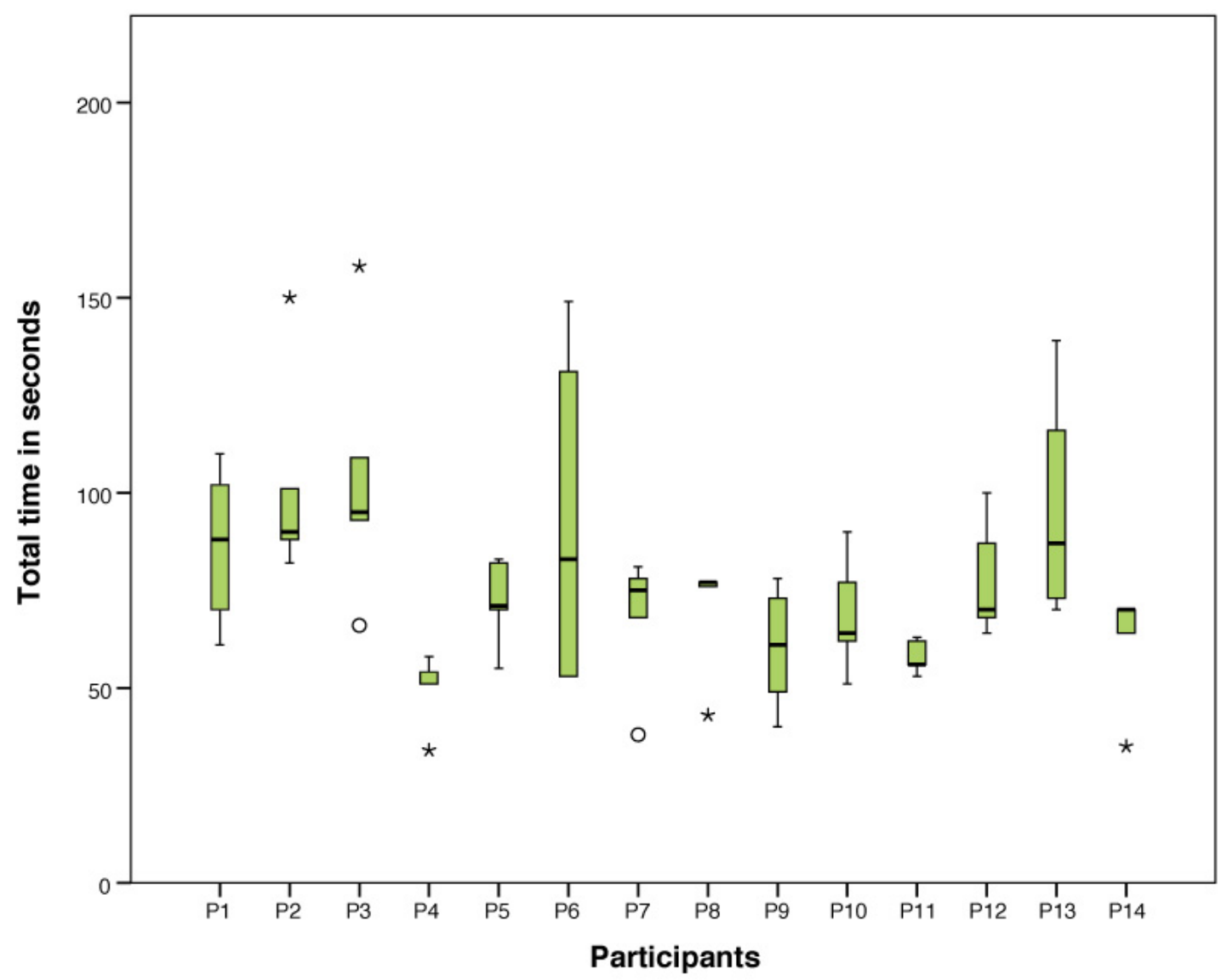

Figure 5.6: Reading times per day for each participant. 


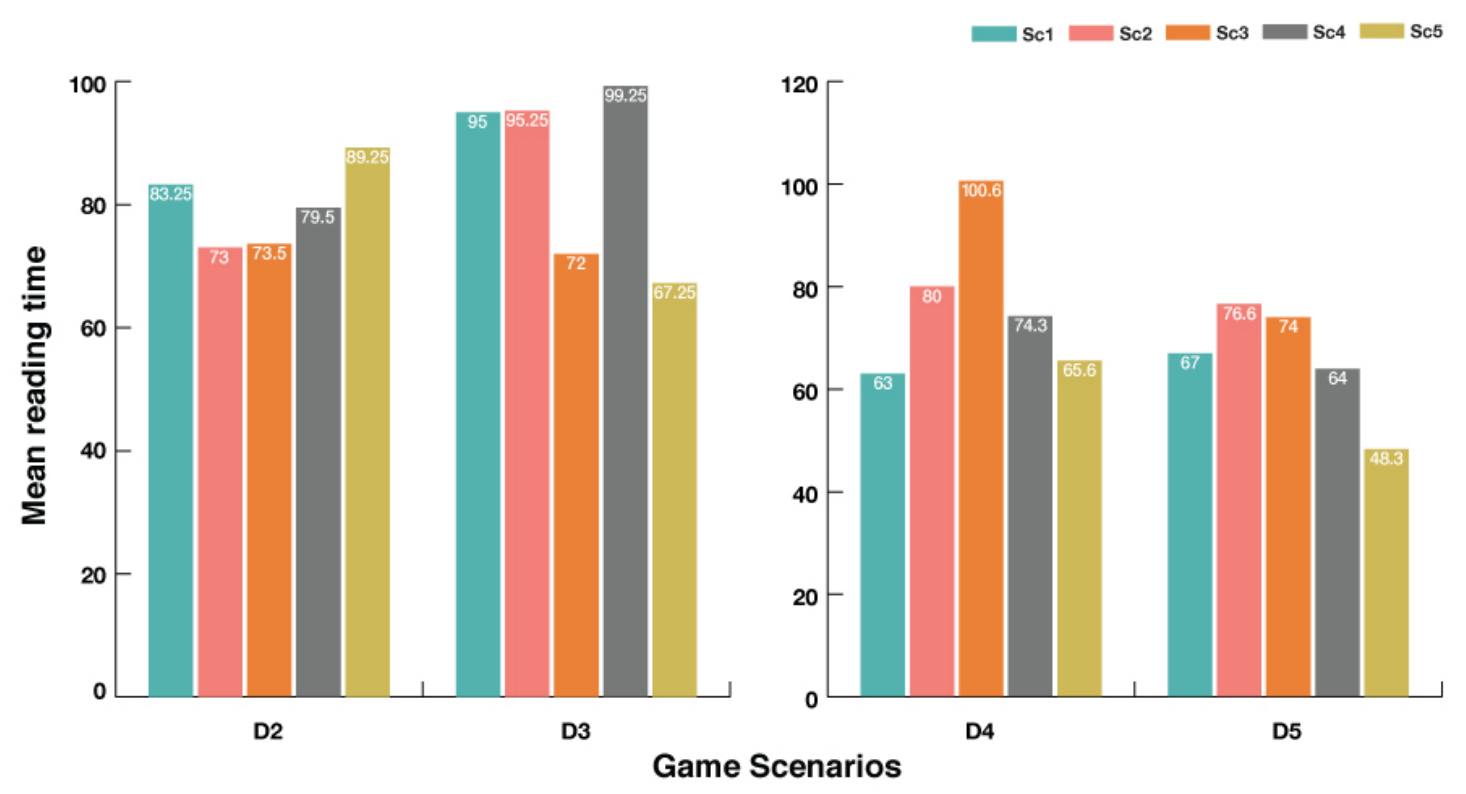

Figure 5.7: Mean reading times per scenario for each of the game days.

\subsubsection{Reading Times}

We analyzed the reading times for D2 through D5. Reading times for D1 were unreliable due to glitches with the prototype.

Figure 5.6 shows the total reading times per participant for each game day. Figure 5.7 shows the reading times per scenario. We found that reading times varied considerably between participants. Times for both individual participants and days had high variability, as indicated by the presence of few outliers and extreme outliers. Participants took between 50 to $104(M=76.45, S D=17.43)$ seconds to read a scenario on average.

\subsubsection{User Feedback Questionnaire}

The user feedback questionnaire was the same as the one presented to participants of the JCJF user study. It covered seven aspects of the game: 1) content length, 2) game scenarios, 3) game play, 4) learning, 5) game fun, 6) game design, and 7) game recommendation. Figures 5.8 through 5.14 illustrate the distributions of responses.

Content length: Most participants were comfortable with the length of the content. All but one participant ranked the content length neutral or above (e.g., 
short) $(M=3.71, S D=0.99)$. When asked verbally, participants mentioned that they are comfortable with the content length of the scenarios and they would not change it.

Game scenarios: The majority of participants described the game scenarios as interesting (10 out of 14) ( $M=3.85, S D=1.09)$, while three participants considered it neither boring, nor interesting. Some of the participants' comments were: "I really like how it follows the Jos around through the events of their day." And: "I like how it is actual situations that kids their age would face" (P2N, P14R).

Game play: Most participants enjoyed playing the game (10 out 14$)(M=$ 4.07, $S D=0.99$ ), while three thought it was borderline boring. Two of the three participants were returning from previous user studies and were now 13 years old, which might explain why they thought it boring, since the game is intended for younger children. One participant (P6R) expressed how much she liked the game and was "addicted to it" and wanted to play it again and try all the available options. She even asked whether the game will be available online and wanted to be notified when that happens.

Amount of learning: Participants were asked to rate their learning experience, and to try to quantify how much they learned. Responses were fairly evenly distributed across the scale. Participants tested only a subset of days, so some may have experienced a game topic that they had already learnt about somewhere else. This variability $(M=3.35, S D=1.21)$ coincides with the results of the pre- and post-game-day interview results, where on the first question (Figure 5.2), participants scored higher in the post-game-day questions on only two of the five game days. However, on the second question (Figure 5.3), there were much more significant improvements.

Game fun: Participants enjoyed the game. The majority rated playing the game as "Fun" (13 out 14) $(M=4.42, S D=0.64)$, while only one participant rated it as neutral.

Game design: Participants appreciated the game design $(M=4.35, S D=0.63)$. P1N for example commented on how Jo's facial expression looked sneaky and said jokingly, "he looks like he's up to something." Another participant appreciated that 
Content Length

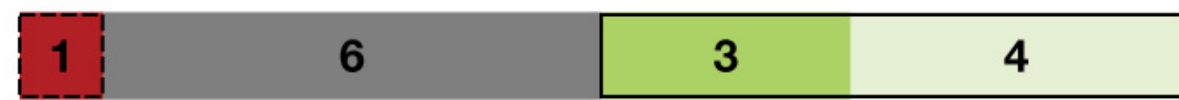

\section{Long $\begin{array}{llllll}1 & 1 & 2 & 3 & 4 & 5\end{array}$ Short}

Figure 5.8: Distribution of Likert scale response for "The content was: Long to Short".

\section{Game Scenarios}

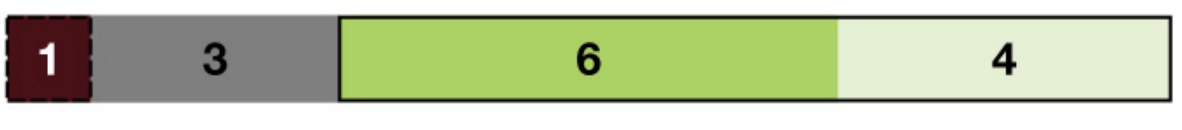

Boring \begin{tabular}{llll|l|l|l|l|l|l}
1 & 2 & 3 & 5 & Interesting
\end{tabular}

Figure 5.9: Distribution of Likert scale response for "The game scenarios were: Boring to Interesting".

the girl character had equal part in the actions and choices.

Recommend game: Half of participants $(M=3.64, S D=1.21)$ would recommend the game, four participants were neutral, and three participants chose the closer to never option. When asked verbally, they mentioned feeling awkward suggesting the game to a friend, saying, "my friends have different taste than me," and "I think it suits younger children more." Some would suggest it to a school teacher rather than their friends as it feels awkward to tell their friends: "here play a game on cyberbullying" because their reaction might be "why are you suggesting this game to me?!"

\subsubsection{Eye Tracking Data Analysis}

Using the eye tracker, we recorded the participants' gaze through the game play. We particularly looked for game elements that caught their attention, examined whether participants read or skimmed text within the game, and explored if new game elements like the score and the newsfeed had any effect on the participants' attention.

Game elements: Figure 5.15 shows P3N's eye tracking scan path for the game's 


\section{Playing The Game}

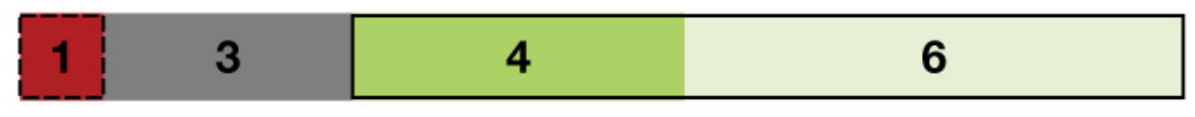

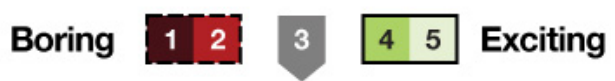

Figure 5.10: Distribution of Likert scale response for "Playing the game was: Boring to Exciting".

\section{Amount of Learning}

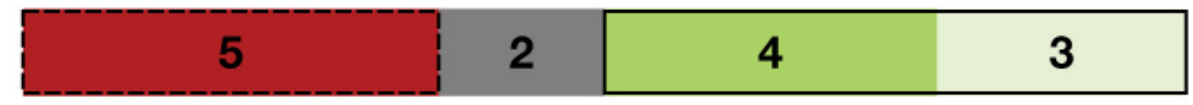

\section{$\begin{array}{llllll}\text { Nothing } & 1 & 2 & 3 & 5 & \text { A lot }\end{array}$}

Figure 5.11: Distribution of Likert scale response for "How much did you learn? Nothing to A lot".

\section{Game Fun}

\begin{tabular}{|l|l|}
\hline 1 & 7 \\
\hline
\end{tabular}

\section{Boring \begin{tabular}{lllllll}
\hline 1 & 2 & 3 & 5 & Fun
\end{tabular}}

Figure 5.12: Distribution of Likert scale response for "The game was: Boring to Fun".

\section{Game Design}

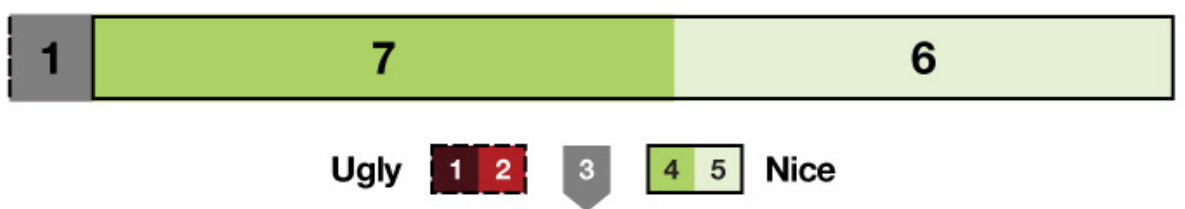

Figure 5.13: Distribution of Likert scale response for "The game design was: Ugly to Nice". 


\section{Recommend Game To Friends}

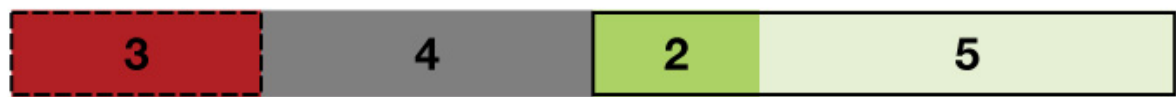

\section{Never \begin{tabular}{llllll}
\hline 1 & 2 & 3 & 5 & 5 & Absolutely
\end{tabular}}

Figure 5.14: Distribution of Likert scale response for "Would you recommend this game to a friend? Never to Absolutely".

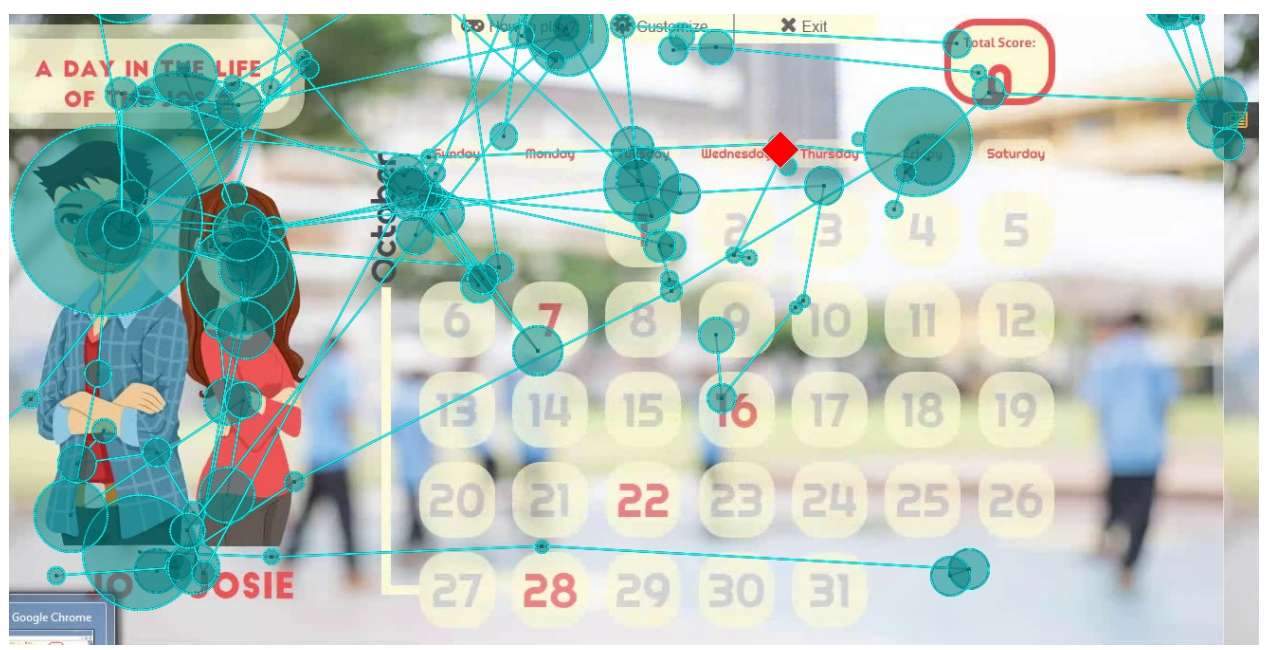

Figure 5.15: Heat map showing a participant's gaze at the game's main and secondary design elements.

main landing page. It illustrates how participants were heavily drawn to the faces of the two main characters, Jo and Josie. From the scan path, we notice the participant scanning all the main elements of the page. They glanced quickly at the score and at the newsfeed tab (not knowing what it is at this point, because they have not played the game yet). They also glanced at the "How to play?" tab at the top of the page.

Colour and Design: Figure 5.16 shows a heat map of a secondary scene from a scenario for P14R. They spent considerable time reading the scenario presented on screen. Other details of the scenario also caught their attention, such as the team's logo and the silhouettes of the basketball players to the left of the text. The participant also paid attention to general game elements like the game's logo and the newsfeed tab. Finally, we notice attention on the background, as if the participant was taking a break from reading or deciphering details of the blurred image background. 


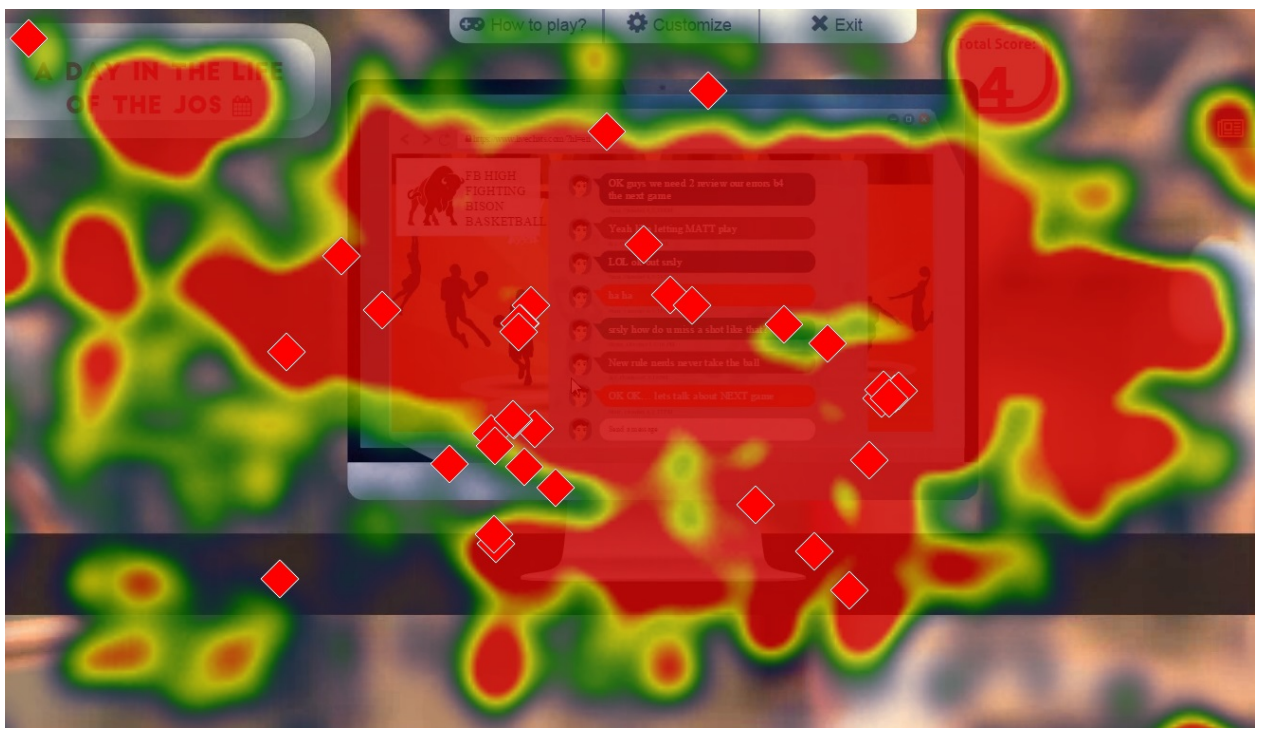

Figure 5.16: Heat map showing a participant focusing on the game content.

Animation: Participants were drawn to the game's animation. For example, Figure 5.17 shows the scan path of the opening scene of D1-1 with Josie in a classroom. Her phone, located on her desk, vibrates when she receives a text message. The scan path shows attention shifting between Josie's face, the scenario's text and the phone as it vibrates. Additionally, we gathered from the participants' verbal feedback that he liked the animations and wished to add more animation throughout.

Game mechanics: Two major game mechanics are the scoring system, present on the upper right, and the newsfeed, accessed by clicking on the right tab.

Some participants showed great interest in their game scores and how well they did within the game, tracking the score they received from one scenario to the other. This is evident from Figure 5.18. We notice that the score is one of the main items that the participant (P14R) examined. Participants' verbal comments throughout the game provided further support for interest in the score. P6R exclaimed, "Oooh, I got a score point!"; and when she received a low score, she was eager to know why and how she can improve her score. P10N also showed great excitement after receiving two points for the first couple of choices. He was similarly eager to know which were the highest score options when he did not receive them.

The newsfeed tab gathered attention from some participants even before they started playing the game and knowing its full function. Figure 5.16 shows P14R's 


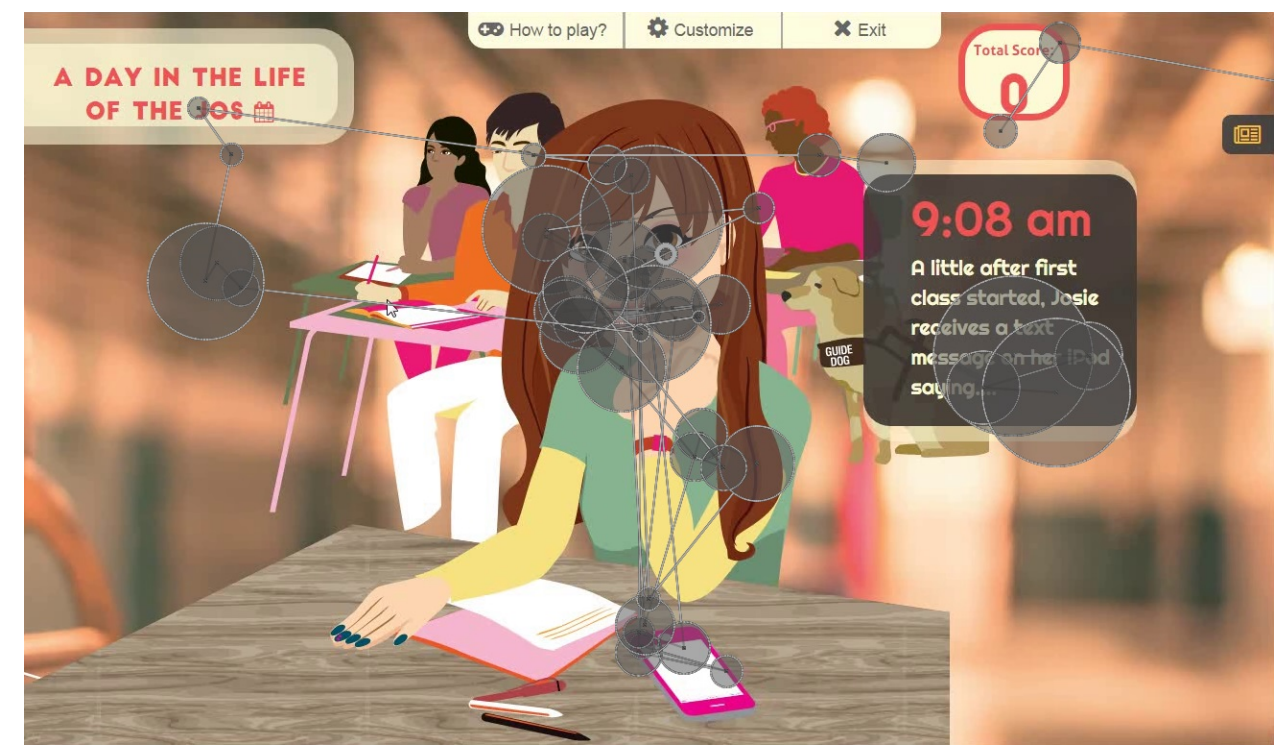

Figure 5.17: Scan path of P7R's gaze towards Josie's phone as it starts to move, indicating that Josie received a text message.

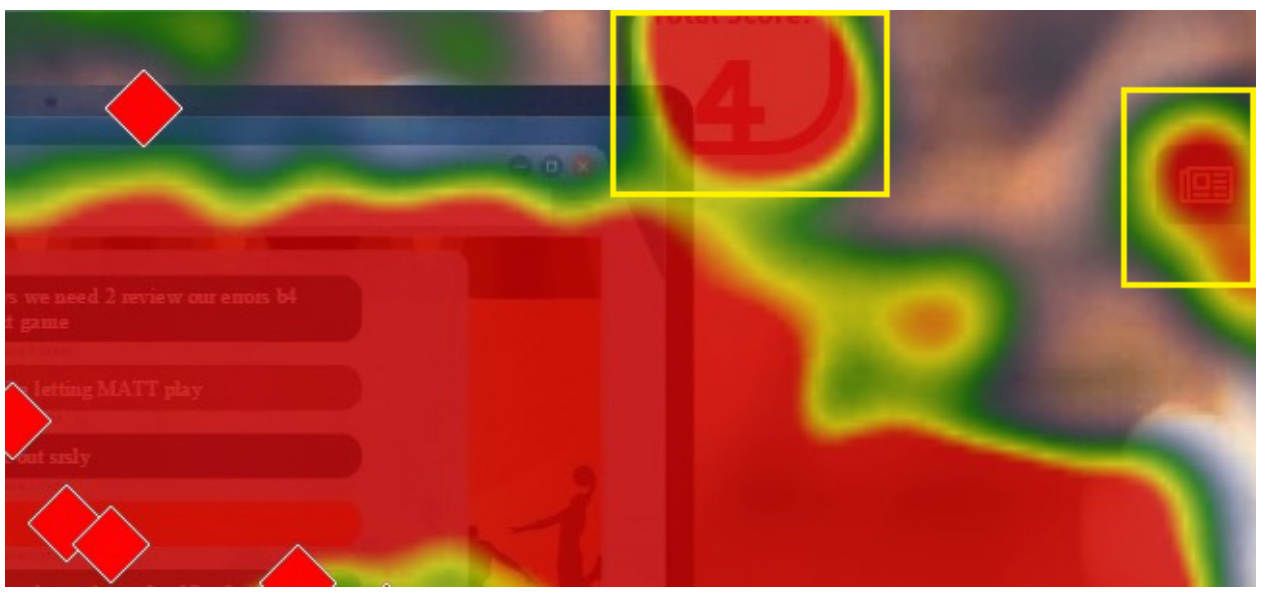

Figure 5.18: Portion of heat map showing P14R's gaze on the score and the newsfeed. 
gaze on the newsfeed tab (red cluster at the right side). Figure 5.19 shows how P5N frequently looked at the newsfeed, as if trying to identify its function before playing the game. Figure 5.20 is a heat map showing P9N gazing at the content of the newsfeed tab after they made their game choice. The newsfeed tab, similar to a social media feed, presents the most current posts on the top and as new feeds come in, the information is pushed downward. The participant focused on new posts as they appeared. Worthy of mention, P9N's heatmap (Figure 5.20) shows the participant focusing only on the text of the most recent posts, and not the associated faces. This contradicts our earlier observation that participants were generally attracted to faces. In this particular instance, we needed to repeat the scenario again in order for the participant to be able to read the consequences on the newsfeed, because of the animation speed glitch discussed previously. The participant was focused on reading the posts as they quickly appeared.

Reading patterns: Line-by-line: Similar to JCJF, we found two different reading patterns. The first is the line-by-line pattern, where the participant spends their time reading one line after the other. Figure 5.21 shows how P10N primarily read from left to right moving down to the next line, until they finished all the text on the screen.

Zig-zag: In the zig-zag reading pattern, the participant switches back and forth between the reading text and looking at other game elements. Figure 5.22 scan path illustrates P13R's zig-zag reading pattern.

Dense text areas: Participants did not read dense text. For example, P1N mentioned that she figured the game text is not important to read, so she skipped over it. Figure 5.23 demonstrates P8N's scan path for a page with dense text. The participant focused on the task at hand and ignored any secondary information that did not advance a particular task.

\subsubsection{Qualitative Feedback}

At the end of the session, we asked for feedback about the game. The questions focused on what they liked and disliked, suggested improvements to the game, whether they would recommend this game to friends, and whether they would like to play the 


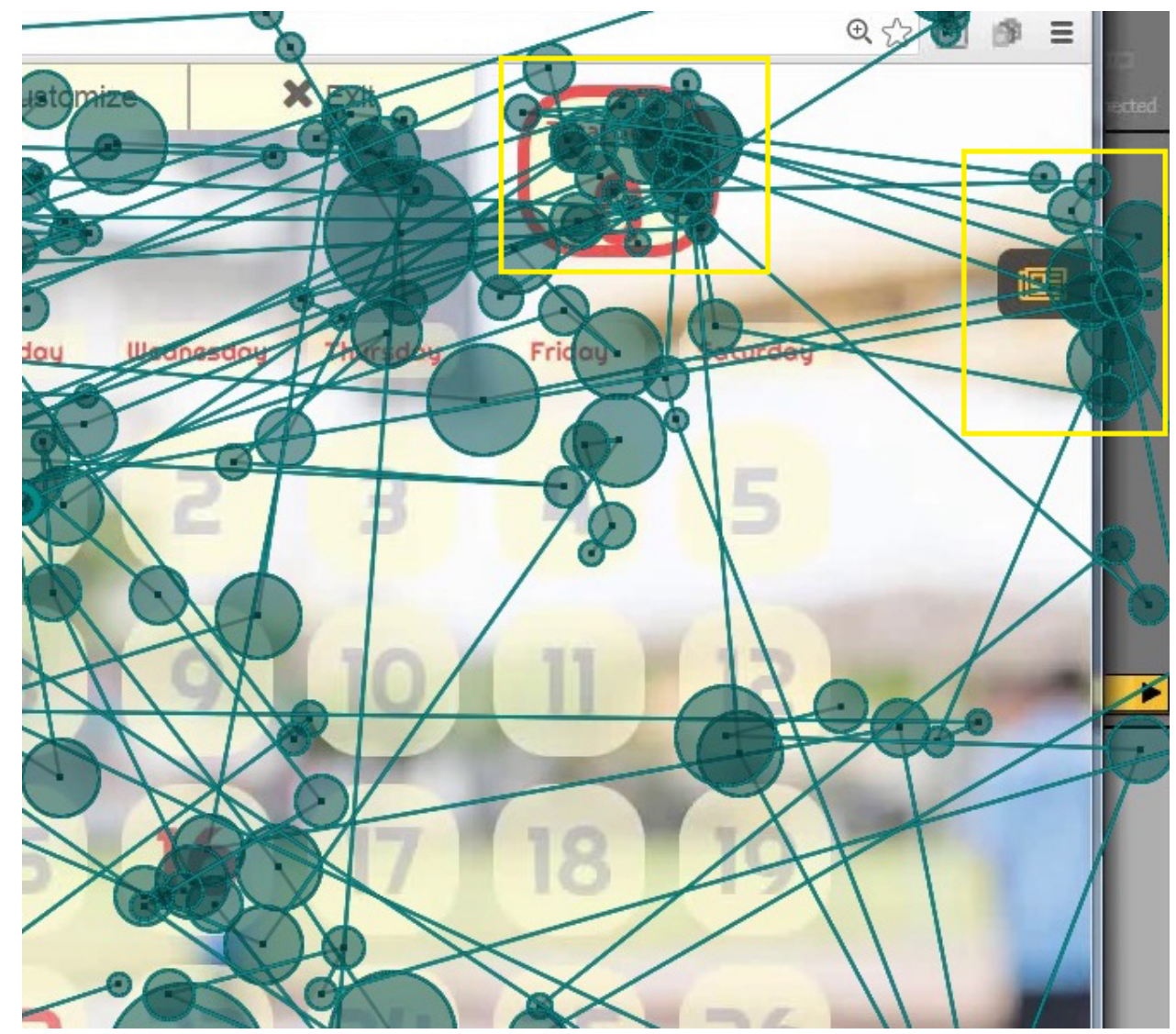

Figure 5.19: Scan path shows P5N looking repeatedly at the newsfeed and score (yellow boxes) on the main page.

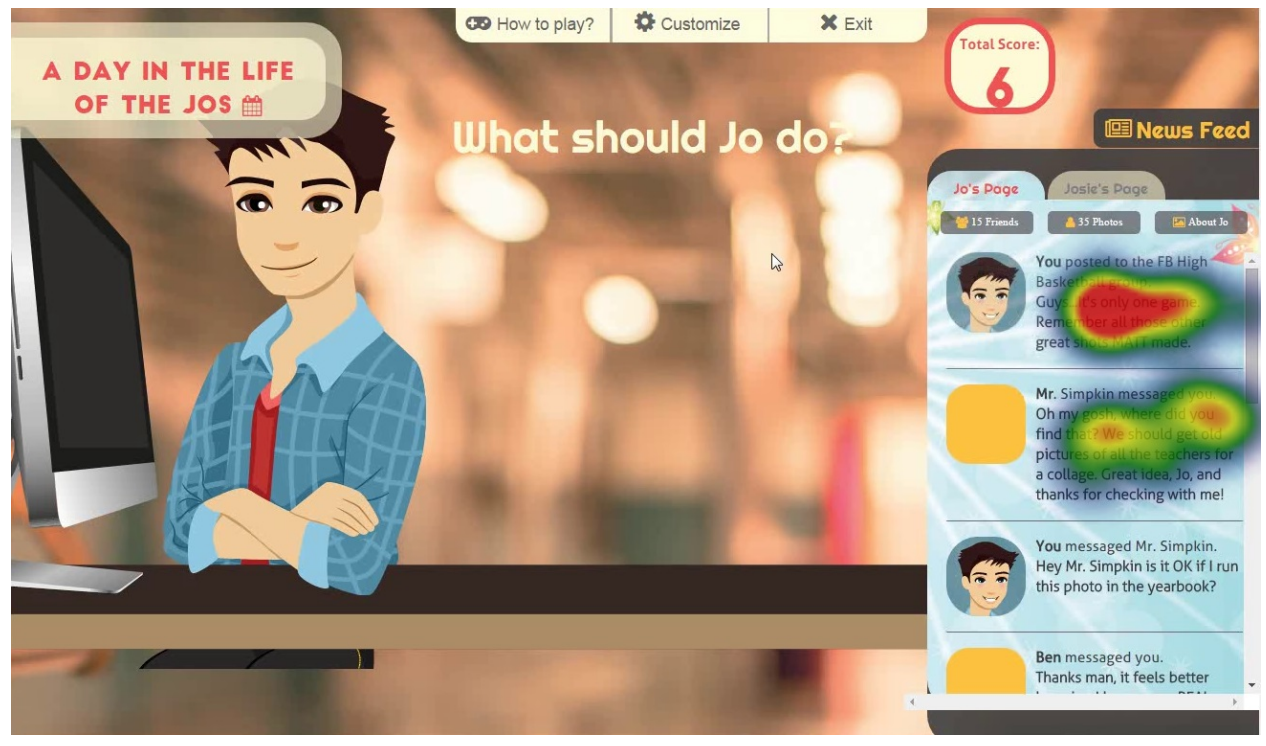

Figure 5.20: Heat map showing P9N focusing on new social media posts. 


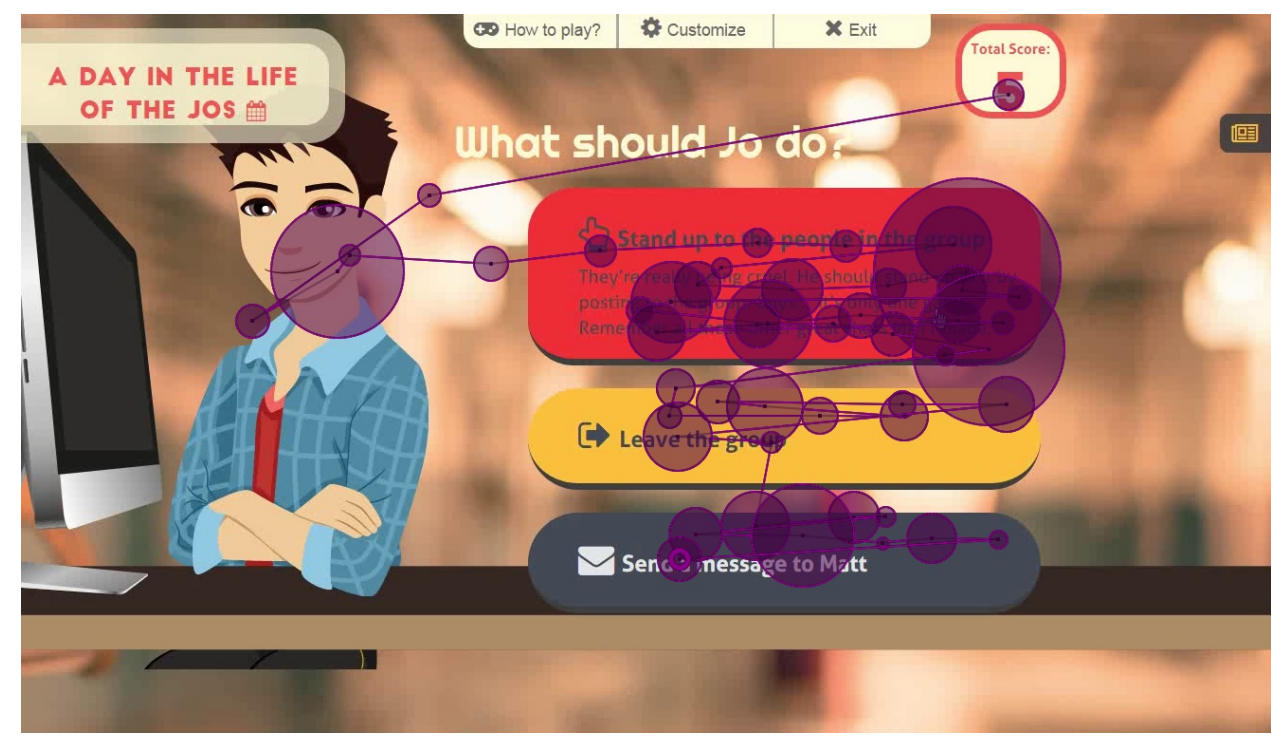

Figure 5.21: Scan path showing P10N's line-by-line reading pattern.

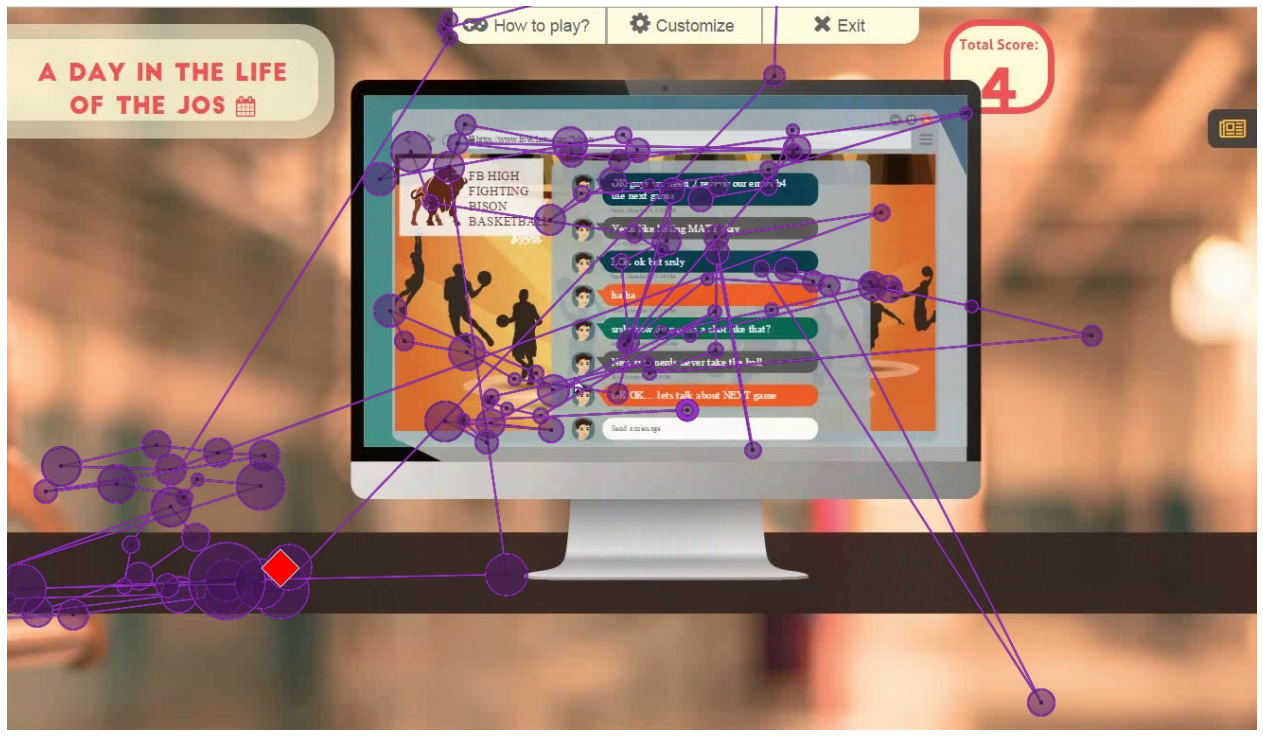

Figure 5.22: Scan path showing P13R's zig-zag reading pattern. 


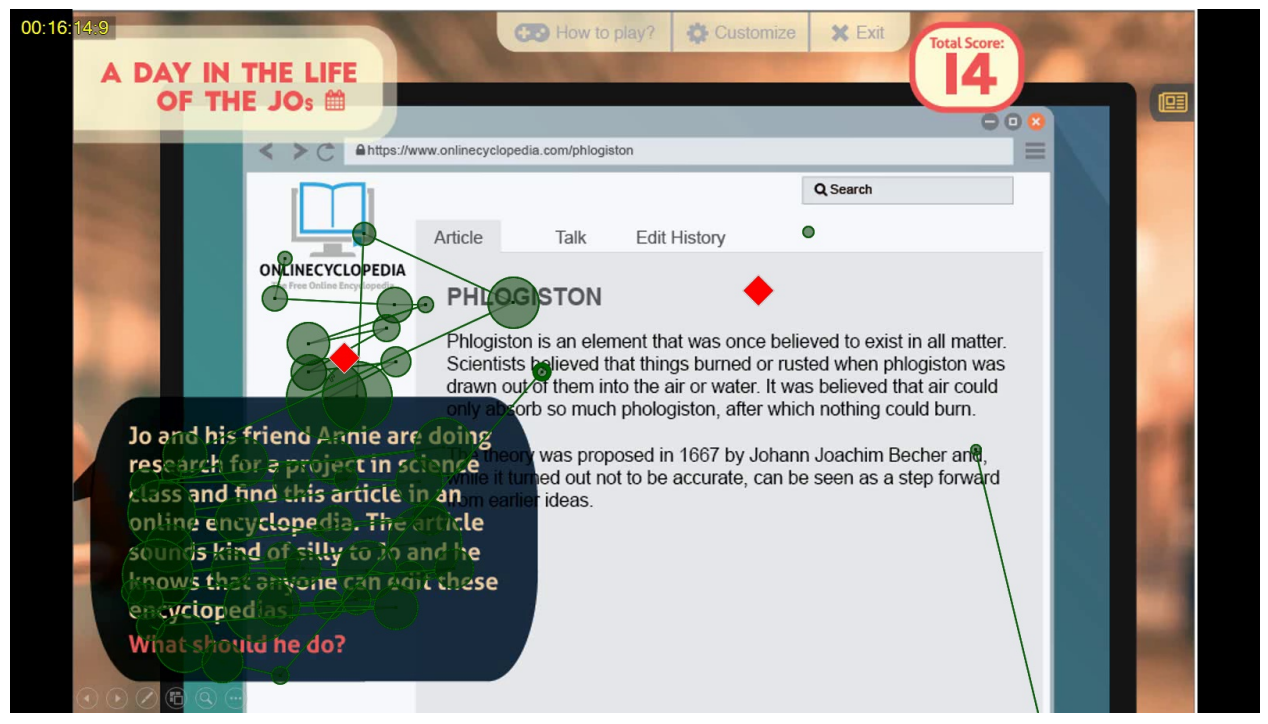

Figure 5.23: Scan path showing how participants only glanced quickly at the long "fake" textual description.

game within a classroom setup. Table 5.3 highlights some of their answers.

Likes: Participants generally liked most of the game. However, some aspects stood out more than others. For example, they particularly liked how the game showed current events using current smart devices and online social media sites like Instagram. Participants also liked how it uses current language that the children of this age group use, such as abbreviations (P6R).

Participants also liked how the game introduces new characters, and not just the brother and sister (P7R). Another participant liked how it is not just a boy (Jo) who is the main character of the game, but also a girl (Josie) (P4N).

The animation was another game aspect that the participants liked (P2N). P6R commented on how she liked the animated consequences that appear on the social media feed once a choice was made. In general, participants thought the game with its animation was a cool way to learn new things about the different game topics. The game scenarios and the ADITL concept was another aspect they enjoyed. Participants enjoyed the setup and how you follow the Jos from class to class (P2N, P11N). P14R appreciated hearing relatable situations in the scenarios. Additionally, participants thought that the format educates kids on different ways to respond to tricky situations (P14R, and P11N). P6R admired how the game explains the reasoning behind the 
different choices. P9N enjoyed the game choices and how each choice has an associated score.

P11N particularly commented on the game design and that she liked how the characters were modelled. P7R, who also tested the old game, preferred the new design a lot more.

Dislikes: Participants disliked the speed of animations on D1, the live version of the game. Many participants thought the animation speed was fast when it should have been slow and slow when it needed to be fast (P2N, P6R, P7R, P9N, and P11N). Other participants expressed no notable issue with the game; for example, P14R said that there is nothing that he dislikes about the game.

Things to improve: Some participants suggested adding pause and next buttons to control the animation speed and to allow for easy navigation from one scene to another (P6R, P7R). Others suggested slowing down the animations to allow participants to read the text in some scenarios (P2N, P9N, P11N).

P5N and P7R suggested adding more animation and making objects like small buildings look more realistic. Some suggested adding a voiceover for the Jos and background music.

Participants objected to "unacceptable" behaviour by the main characters. Two participants commented on the first scenario in D1 - cyberbullying and how Josie should not be texting in class, "Well first of all Josie should stop texting in class!" and "Texting in class is not okay." Others commented on how Jo should not be taking pictures while he's riding his bike (P3N, P6R).

Recommend to a friend or play in a classroom: Some participants would recommend the game to a friend (P4N, P2N, P8N, P11N). One participant explained that it would be awkward to suggest the game to a friend of their age group. They feared that their friend might think that they are trying to give them a hidden message (P6R). An older participant mentioned how they would not recommend it to their friends in Seventh grade but maybe to kids in elementary school who don't know about this information (P7R). Another would recommend the game to a friend if that friend was having trouble with a particular game issue (P14N). Others would recommend it to teachers of their own school grade or younger (P6R, P7R). 
When participants were asked if they would like to play this game within a classroom, some agreed but thought that the teacher will have to enforce rules to prevent the students from surfing the Internet or doing other non-game-related activities online (P9N, P11, P13R).

\subsubsection{General Observations}

We observed two main points of frustration with the high-fidelity prototype for D1. Both issues will be addressed in the next iteration of the game. When playing D1, participants were frustrated by its animation speed. Almost all participants had to repeat some scenarios because the scene changed so quickly that they could not read all the text. We had to repeat some scenes three or four times for some participants. Another frustration was the small size of some text, as a result of cross-platform issues with the prototype.

Aside from those two frustrations, participants generally showed great excitement when they received high game scores, and were eager to read the text explanations when they made choices with lower scores. Some participants positively remarked on the Jo's facial expressions. For example, P1N commented "He looks like he's up to something. He has a sneaky smile on his face." A returning participant (P6R) commented how she preferred Jo without the pink cheeks (from Chapter 4 designs) because this made him look less like a "teacher's pet." The same participant also commented on how cool Josie looks.

We found that some participants were not compelled to read the end-of-day summary for the live D1 prototype. The summary might look unattractive when compared to the rest of the game elements since it is text-heavy and displayed in plain text on a white background. This is problematic because the summary reenforces the educational lessons conveyed within the game and is considered an important pedagogical component by MediaSmarts. 


\section{Game Design}

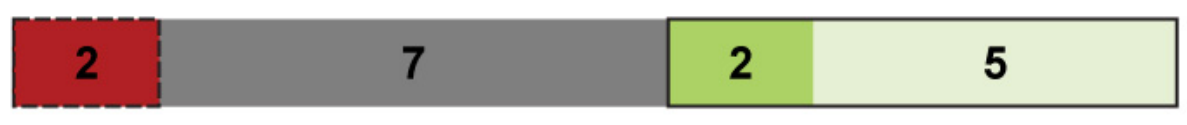

Ugly \begin{tabular}{llll|l|l|l|l|l|l|l|l}
\hline 1 & 2 & 3 & 5 & Nice
\end{tabular}

Figure 5.24: Likert scale response for "The game design was? Ugly to Nice" for JCJF.

\section{Game Design}

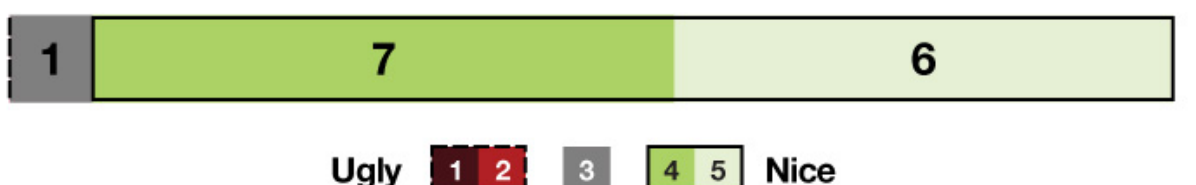

$\begin{array}{lllllll}\text { Ugly } & 1 & 2 & 3 & 4 & 5 & \text { Nice }\end{array}$

Figure 5.25: Likert scale response for "The game design was: Ugly to Nice" for ADITL.

\subsection{Comparison with old game}

\subsubsection{Visual appeal}

Overall, participants prefer the look and feel of ADITL. This is apparent from the verbal feedback, and the survey responses for questions repeated for both JCJF and ADITL. Figure 5.24 and Figure 5.25 show participants rating the ADITL higher than JCJF's when asked to rate the game design between ugly and nice. Verbal feedback from the two studies also highlight a preference for ADITL's visual design. Participants of the JCJF study found that content was displayed in an unappealing and "dumb" way that needs to look "a little newer". P7R specifically mentioned how he liked the overall look-and-feel of ADITL better than JCJF. Participants particularly were excited about the presence of a game score and they followed it from one scenario to the other.

\subsubsection{Game topics}

Section 3.4.5 discusses participants' concern regarding JCJF's content. P10N and P6R felt that online dating was more appropriate for older teenagers (18 and older). 
P10N felt that introducing the concept of plagiarism and copying essays might encourage previously unaware children to try it out. Other participants commented on how JCJF is outdated and dealt with older technologies like burning CDs. We further noted that the game does not address issues like social media and handling of mobile devices.

Participants of the ADITL study particularly liked how the game uses current events and the devices throughout the game scenarios. The use of online social media sites like Instagram and mobile devices was appealing to them. Participants also commented positively on the use of familiar language. Other than noting that Josie should not text in class and Jo should not take photos while riding his bicycle, no participant expressed concern about the content of ADITL. Likert-scale responses about game scenarios (Figure 3.9 and 5.9) were very similar for both games.

\subsubsection{Game elements}

One of the missing elements in JCJF was the gamification. The main interaction and feedback loop in JCJF was finding out if their choice was a "cool" or "fool" choice. We incorporated two new elements, a scoring system and a newsfeed. Section 5.2.5 shows how participants were particularly excited about their game scores. Figure 5.18 and Figure 5.19 show how the score and the newsfeed icons catch the participants' attention in the eye tracking data. Verbal feedback shows that participants were eager to know if they have earned points or not. For example, P6R shouted, "Oooh, I got a score point!" when she got a right answer and P10N exclaimed "I got a score point, cool!" In D2 - D5, participants were handed the results of their choices on paper, instead of reading them on the screen. We noticed that participants were eager to read the rest of the choices' results to see why their choice did not earn them the highest game score. From our observations, we find that a score helped keep participants engaged in the game.

The newsfeed is another element that caught participant's attention. Figure 5.20 shows how participants spent time reading through the different posts appearing on Jo's newsfeed after the participant had made their choice. The newsfeed highlights consequences of their actions as experienced by the game characters, providing more 
context than lecturing about appropriate behaviour.

Although JCJF included some animation in the sense of a blinking button or text, we designed ADITL to include more animation. For example, the newsfeed pops open when the participant makes a choice, with different posts appearing one after the other. Other animations incorporated within the scenarios, such as Josie's phone vibrating in D1-Sc1 as she receives a text message from her friend Sofia. The screen then animates to show a closeup on Josie's phone and messages start appearing on it one by one. Figure 5.17 shows participant's attention shifting from Josie's face to her phone as it started vibrating on her desk. Verbal feedback also shows that participants were excited about ADITL's animation although some minor modifications are needed to improve usability.

\subsubsection{Reading Times}

Since participants found JCJF's text tool long, we actively reduced text that in the ADITL scenarios. Reading times were cut nearly in half. Averages for JCJF ranged from 99 seconds to 378 seconds compared to 50 to 105 seconds for ADITL. As for the verbal comments, we often heard comments like: "do I have to read this?" from the JCJF participants, but ADITL participants did not complain about the length of the text. When we asked participants of ADITL about content length, they said they were comfortable with it.

\subsubsection{User feedback questionnaire}

In general, ADITL scored similarly or higher than JCJF in all Likert-scale questions. ADITL participants thought that the game was more exciting (Figures 3.10 and 5.10), that they learned more (Figures 3.11 and 5.11), that the game was more fun (Figures 3.12 and 5.12), and that the game design was nicer (Figures 3.13 and 5.13).

We found no improvement in participants' perception of content length (Figures 3.8 and 5.8), or game scenarios (Figures 3.9 and 5.9). We also saw no difference whether they would recommend the game to friends (Figures 3.14 and 5.14). 


\subsubsection{Amount of learning}

Generally we saw more improvement in participants' knowledge from playing ADITL than JCJF. A direct comparison in the pre- and post-game questions is not possible because ADITL contained changes in the game design, which meant that we were not able to ask the same questions.

Mainly, there were more noticeable increases in knowledge in ADITL; we saw increases in knowledge in 7/10 questions (Figures 5.2 and 5.3). In JCJF, we saw an increase in 7/16 questions (Figure 3.4). In ADITL, the answers showed greater variability in the pre-game viewing, particularly for question 2, indicating that participants were not as familiar with the material ahead of time. However considerable increases in knowledge were seen after playing the game, with participants giving more complete answers that reflect a wider range of actions if faced with a difficult situation. 


\section{Chapter 6}

\section{Discussion and Conclusion}

\subsection{Discussion}

\subsubsection{Children as study participants}

In this section we look closely at areas pertaining to testing with children versus testing with adult participants. We observed the following notable differences:

Limited attention span: We noticed that when dealing with children they exhibited limited attention span especially if the material was not very engaging. Slow readers in particular exhibited lower attention span than fast readers, especially when faced with dense and long pieces of text to read. We recommend keeping the study sessions short, a maximum of 30 minutes long. We also recommend designing the study to include smaller tasks, and maintaining flexibility in how much to test

with each child. If the child starts to show signs of agitation or loses interest, it is best to cut the study short rather than have the child half-willingly participate.

High expectations for the prototypes: During our user studies, we found that children have low tolerance for interfaces that were not operating according to their expectation, or that contained glitches. They complained that some of the game's features were not behaving as they would expect based on experience with their other games. They were annoyed by certain aspects of the game that were outdated. They expected a high degree of interaction and polish in the interface, even though we shared with them that this is not the final version of the game. We find this very different from adult participants, who, when warned that this is a first version of the app, acknowledge this fact, accept it, and are able to give feedback while taking this into consideration.

Novelty effects: We found that with children, the novelty effects [66] were high. Novelty effects occur when participants have a more positive impression of an 
interface because it is their first time seeing it. This was evidenced especially with the returning participants; they gave high ratings the first time around, but showed less enthusiasm and gave equal or lower ratings by the third user study.

Response bias effect: Response bias occurs when the participant alters their answer to fit within a socially desirable response [62]. Children, in particular, are affected by the response bias, since it is in their nature to want to please the adults in their lives, especially when they are younger. Overall, we noticed that when children were dissatisfied with a specific aspect of JCJF and ADITL, they would do one of two things. They either mentioned it in a very subtle way or did not mention it at all. They would say that overall they liked the game and gave only positive feedback. However, if we probed with questions, they would eventually declare their true opinion. For example, one participant said he liked JCJF, however, when probed, said he would not have played the game if he came across it on his own.

Maturity level varied significantly: We found that maturity levels varied significantly between children of different ages. Children who were 10 years old exhibited much less sophistication in thinking and knowledge of cybersecurity than those who were 11 years old. Participants who were nearly 13 gave more sophisticated answers. Anecdotally, children who were the oldest sibling appeared more mature than those of similar age who were youngest in their family. We also note that adults, in general, moderate their feelings more than children. Children's responses were significantly affected by their mental, emotional, or physical mood.

Availability controlled by parents: We found that despite our effort to ensure verbal assent, children had little control over their ability to participate in the user study. If the study timing or duration conflicted with the family's schedule then the parent would alert us that the child cannot participate because of another commitment. Coordinating around these complex schedules was challenging. Adult participants have the freedom to decide for themselves, and typically have more flexibility in scheduling. 


\subsubsection{Children's Interaction With Games}

Age differences: We found that the initial target age range for both JCJF and ADITL was inaccurate. For JCJF, the target age range was from 11 to 14 years old, but we found that JCJF was not quite suitable for younger children and was boring for the older children. For ADITL, we narrowed the age range to 11 and 12, but, we found that children that were nearly 13 were bored with the game and thought it not stimulating enough.

We also found that children's development varied greatly between 11 to 12 , and 12 to 13 year olds. We noticed great difference in interest and knowledge, with some participants clearly still children while others were more like teenagers. We recommend selecting the target age range to be narrow, in order to better cater to their needs and abilities.

We also found that children of this age range have strong opinions of the game topics and a strong sense of right and wrong. Throughout the studies, children commented that different topics were not appropriate to their age group, and that particular behaviour of the Jos was not acceptable in the given circumstances.

Relating to game characters: Children ascribed human attributes, feelings, and characteristics to game characters. They easily connected and commented on the Jos' and their peers' actions as if they existed in real life. They were invested in the characters, and were willing to suspend disbelief. Facial expressions of the characters impacted the children, as they cued the overall feel of the scene from the Jos' facial expression. We found that the connection the children made with the game characters seemed particularly beneficial for remembering lessons and understanding the impact of their game choices on the Jos' life. The children were able to understand at a deeper level the lessons learned, and reflect on actions and consequences by placing themselves in the characters' position.

Gender balance: Girls, in particular, appreciated having a heroine with strong role as a main character like Josie. They were pleased and commented on equal presence in the game, which might further indicate that they saw themselves in Josie and her female friends. We found that the boys did not mention or appear to notice this strong female presence, and there were no signs that they were bothered by it. 
We believe that it is important to include diversity in representation of characters, not only for different genders, but also from different ethnicities, and with varying abilities.

Appropriateness of game mechanics: Out of the game mechanics, the score stood out as the most effective tool to encourage the children towards learning. We saw visible attachment, cheering, and disappointment when children received a game score, and when they did not receive the highest score for a scenario. In D2 - D5, where we handed the outcomes as plain text printed on sheets of paper, we noticed the children were eager to read why a different choice received a higher score. We heard them exclaim, things like "really, why?!", which indicated curiosity when they thought their choice had been the best answer. Scores are an extrinsic learning motivators in a game [29]. Although the literature recommends relying on intrinsic motivators for learning [73], we found that the score augmented the learning experience, rather than degrading it.

For the newsfeed, we did not notice an immediate reaction. We suspect that its placement and glitches in D1's prototype meant that the children were not as excited about it and that its purpose was not obviously noticeable to them. We will assess it more thoroughly in future prototypes.

Humour was most successful in visual form (e.g., facial expressions). It could be due to the limitations of the prototype, but participants were having difficulty reading the text and extracting the humour in it. We may also find in later evaluations that children favour visual stimulations over reading text generally.

For the consequences, again the children were most motivated by the impact on their score, rather than the posts that appeared on the newsfeed. This could be partially attributed the fact that the children did not control the animation speed and the text might have looped too quickly for them to catch what had happened on the Jos' newsfeed.

Use of current technologies: Children were impressed with the (mock) apps and devices that showed up in the different scenarios of ADITL. While positive, this suggests that for the game to stay current in the future, it will need constant updating of the scenarios and scenes in order to reflect changing technologies. We 
designed ADITL in a way where we could swap out individual scenarios, scenes, or days without reconfiguring the main game structure. This allows for easy updates in the future.

Finally, we relied heavily on the use of social media as feedback mechanism, which is highly meaningful today, but may become outdated in a few years.

\subsection{Recommendations}

Based on previous discussion we have general recommendations when designing educational games for this age group. These are some high-level recommendations that we have extracted from the lessons learned by designing ADITL for this age group.

1. A game should include diversity by showing characters of different ethnicities, and different abilities, as well as gender balance. This was particularly helpful to players represented by the increased diversity, who may not have related as well to the other characters.

2. A game must include a scoring mechanism for this age group. They were most motivated by it and it encouraged them to pay attention to the actual lesson content.

3. A game must stay current, as children have low tolerance for out-dated features.

4. A game should make characters relatable. The children responded well to the characters and it helped them imagine themselves in the scenarios.

\subsection{Limitations and Future Work}

Prototype limitations: Technical limitations and glitches in the prototype impacted the results. The impact appeared most in the user feedback questionnaires of the returning participants, where two rated ADITL's game design lower than what they rated JCJF because it was not fully functional. Additionally, the prototype was only for D1 of the game, which did not allow us to uncover usability issues with other game days. 
Moving forward, we will test ADITL again with a full version of the game, fix the glitches before testing, and address users' suggestions where possible. This will allow us to remedy the usability issues because of the prototype's limitations and allow us to test the game more thoroughly.

Long term knowledge retention: We did not test long term knowledge retention for ADITL. We tested the children's knowledge immediately after game interactions. Moving forward, we will test knowledge retention after longer intervals (e.g., a week or a month) to see how much the children retained from the information they viewed while playing ADITL.

Ecological validity: ADITL will eventually be deployed in a classroom setting, where it will be part of the online privacy and security educational curriculum. Due limitations of prototype and time, we did not test ADITL in its ecological setting. As part of our future plan, our goal is to test ADITL in the classroom. Unlike the lab setting, where the game is the children's only focus and interaction, the classroom will also have the teacher's discussions about the game topic to be played that day. Also, children can have conversations among themselves if interacting with the game as a group.

\subsection{Conclusion}

In the previous chapters, we discussed the literature leading to our research on the use of gamification in children's education, and in particular children's education about cybersecurity and online privacy issues. We conducted a study with MediaSmarts' existing JCJF game to establish a starting point for the design of our new game. Using the ADDIE process, we designed the game mechanics, premise and storyline for the new game and created three potential visual designs. We conducted a pilot study evaluating these designs and established ADITL's direction. We developed a full prototype of Day 1 and medium fidelity prototypes of the remaining days. Finally, we conducted a user study of this ADITL prototype.

Results showed that participants found ADITL more appealing than JCJF. This is evident from participants' high ratings on the user feedback questionnaire, as well as the pre- and post-game interview questions that measure the learning. We also 
discovered that adding gamification elements to the educational learning material increased likability of the game and promoted interaction with the lessons. Moving forward, we will test the final online version of the full game, and we will assess longterm knowledge retention to see whether the game meets its educational objectives. 


\section{Bibliography}

[1] A. Adams and M. A. Sasse. Users Are Not the Enemy. Commun. ACM, 42(12):40-46, December 1999.

[2] S. Adams. Dilbert. http://dilbert.com/search_results?terms=Security. Accessed: 2016-06-11.

[3] R. Alhajj and J. Rokne, editors. Online Privacy Risk Management, pages 11931200. Springer New York, New York, NY, 2014.

[4] M. Allen. Successful e-Learning Interface: Making Learning Technology Polite, Effective, and Fun. Pfeiffer, 989 Market Street, San Francisco, CA 94103-1741, 2011.

[5] L. Bek. Lola Bek Illustrations. http://www. shutterstock.com/g/LolaBek. Accessed: 2016-08-01.

[6] R. K. Branson, G. T. Rayner, J.P. Furman, and F. J. King. Interservice procedures for instructional systems development. executive summary and model. Technical report, Technical report, DTIC Document, 1975.

[7] C. Brigas, C. A. Gomes, M. J. Marcelino, and M. J. Silva. Modeling and Simulation for Elementary Education. In 2009 Ninth IEEE International Conference on Advanced Learning Technologies, pages 468-472, July 2009.

[8] S. Chiasson and C. Gutwin. Design Principles for Children's Software. Number HCI-TR-05-02. Department of Computer Science, University of Saskatchewan, 2005 .

[9] S. Chiasson, M. Modi, and R. Biddle. Auction Hero: The Design of a Game to Learn and Teach about Computer Security. In Curtis Ho, and Meng-Fen Grace Lin, editor, Proceedings of E-Learn: World Conference on E-Learning in Corporate, Government, Healthcare, and Higher Education 2011, pages 22012206, Honolulu, Hawaii, USA, October 2011. Association for the Advancement of Computing in Education (AACE).

[10] J. Combs, S. Edmonson, and S. Harris. The Trust Factor: Strategies for School Leaders. Routledge, 711 Third Avenue, New York, NY, 10017 USA, 32013.

[11] Wikimedia Commons. ADDIE Model of Design. https ://commons.wikimedia. org/wiki/File:ADDIE_Model_of_Design.jpg. Accessed: 2016-07-22. 
[12] A. Danesh, K. Inkpen, F. Lau, K. Shu, and K. Booth. Geney: Designing a Collaborative Activity for the Palm Handheld Computer. In Proceedings of CHI 2001, pages 388-395, 2001.

[13] T. Denning, T. Kohno, and A. Shostack. Control-Alt-Hack ${ }^{\mathrm{TM}}$ : A Card Game for Computer Security Outreach and Education (Abstract only). In Proceeding of the 44th ACM Technical Symposium on Computer Science Education, SIGCSE '13, pages 729-729, New York, NY, USA, 2013. ACM.

[14] S. Deterding, D. Dixon, R. Khaled, and L. Nacke. From Game Design Elements to Gamefulness: Defining "Gamification". In Proceedings of the 15th International Academic MindTrek Conference: Envisioning Future Media Environments, MindTrek '11, pages 9-15, New York, NY, USA, 2011. ACM.

[15] A. Druin, B. B. Bederson, J. P. Hourcade, L. Sherman, G. Revelle, M. Platner, and S. Weng. Designing a Digital Library for Young Children. In Proceedings of the 1st ACM/IEEE-CS Joint Conference on Digital Libraries, JCDL '01, pages 398-405, New York, NY, USA, 2001. ACM.

[16] M. Duggan, A. Lenhart, C. Lampe, and N. B. Ellison. www.pewinternet.orgConcerns About Children, Social Media and Technology Use. http://www.pewinternet.org/2015/07/16/ concerns-about-children-social-media-and-technology-use/. Accessed: 2016-08-17.

[17] Ebay. Ebay Security and Privacy Protection. http://pages.ebay.com/help/ account/protecting.html. Accessed: 2016-06-10.

[18] Facebook. Reporting a Child Under the Age of 13. https://www. facebook. com/help/157793540954833. Accessed: 2016-06-11.

[19] C. Fisher. Designing Games for Children. Focal Press, 2 Park Square, Milton Park, Abingdon, Oxon OX14 4RN, 122014.

[20] J. Flood, D. Lapp, J. R. Squire, and J. M. Jensen, editors. The reader, the text, the poem: the transactional theory of the literary work. Southern Illinois University Press, Carbondale, IL, 1978.

[21] J. Flood, D. Lapp, J. R. Squire, and J. M. Jensen, editors. Handbook of Research on Teaching The English Language Arts, pages 814-824. Lawrence Erlbaum Associates, Inc., Mahwah, NJ, 2003.

[22] T. Fullerton, C. Swain, and S. S. Hoffman. Gamedesign Workshop, pages 49-85. Elsevier, Burlington, MA, 2008.

[23] D. Gelman. Design For Kids. Rosenfeld Media, 57 Third Street, \#4R, Brooklyn, New York, 11215 USA, 72014. 
[24] Google. Google Classroom. https://support.google.com/edu/classroom/ answer/6020279?hl=en. Accessed: 2016-08-15.

[25] Google. Google Classroom Benefits. https://support.google.com/edu/ classroom/answer/6376881. Accessed: 2016-08-15.

[26] Google. Google Safety Center. https://www.google.com/safetycenter/ everyone/start/. Accessed: 2016-06-11.

[27] K. Gori. Kakigori Studio Illustrations. http://www.shutterstock.com/g/ Kakigori+Studio. Accessed: 2016-08-01.

[28] K. L. Gustafson and R. M. Branch. What is instructional design? In Trends and Issues in Instructional Design and Technology, pages 16-25, 2002.

[29] L. Hanna, K. Risden, M. Czerwinski, and K. J. Alexander. The Design of Children's Technology. chapter The Role of Usability Research in Designing Children's Computer Products, pages 3-26. Morgan Kaufmann Publishers Inc., San Francisco, CA, USA, 1998.

[30] Instagram. Instagram. https://www. instagram.com/?hl=en. Accessed: 201606-11.

[31] Internet World Stats. Internet World Stats. http://www . internetworldstats . com/emarketing.htm. Accessed: 2016-05-30.

[32] Ironhide Game Studio. Kingdom Rush. http://kingdomrush.com/. Accessed: 2016-06-11.

[33] M. Johnson, R. Riel, and B. Froese-Germain. Connected to Learn: Teachers' Experiences with Networked Technologies in the Classroom. Technical report, MediaSmarts and Canadian Teachers' Federation, 2016.

[34] N. Kaplan, Y. Chisik, K. Knudtzon, R. Kulkarni, S. Moulthrop, K. Summers, and H. Weeks. Supporting Sociable Literacy in the International Children's Digital Library. In Proceedings of the 2004 Conference on Interaction Design and Children: Building a Community, IDC '04, pages 89-96, New York, NY, USA, 2004. ACM.

[35] K. M. Kapp. The Gamification of Learning and Instruction, pages 1193-1200. Pfeiffer, San Francisco, CA, 2012.

[36] P. Kumaraguru, S. Sheng, A. Acquisti, L. F. Cranor, and J. Hong. Lessons from a real world evaluation of anti-phishing training. In 2008 eCrime Researchers Summit, pages 1-12, Oct 2008. 
[37] P. Kumaraguru, S. Sheng, A. Acquisti, L. F. Cranor, and J. Hong. Teaching Johnny Not to Fall for Phish. ACM Trans. Internet Technol., 10(2):7:1-7:31, June 2010.

[38] J. C. Lester, S. A. Converse, S. E. Kahler, S. T. Barlow, B. A. Stone, and R. S. Bhogal. The Persona Effect: Affective Impact of Animated Pedagogical Agents. In Proceedings of the ACM SIGCHI Conference on Human Factors in Computing Systems, CHI '97, pages 359-366, New York, NY, USA, 1997. ACM.

[39] M. Madden, A. Lenhart, M. Duggan, S. Cortesi, and U. Gasser. Teens and Technology 2013. http://www.pewinternet.org/2013/03/13/ teens-and-technology-2013/. Accessed: 2016-06-11.

[40] P. Markopoulos, J. C. Read, S. MacFarlane, and J. Hoysniemi. Evaluating Children's Interactive Products. Morgan Kaufmann, 30 Corporate Drive, Suite 400, Burlington, MA 01803, 052008.

[41] S. McCloud. Google Chrome. http:www.google.com/googlebooks/chrome. Accessed: 2016-06-11.

[42] S. McQuade and N. Sampat. Survey of internet and at-risk behaviors. Technical report, Rochester Institute of Technology, 2008.

[43] MediaSmarts. A Day in The Life of The Jos. http://mnet.hypernet.ca/ Jo-html5/index.html. Accessed: 2016-07-16.

[44] MediaSmarts. Click if You Agree (Grades 7-9). http:// mediasmarts.ca/digital-media-literacy/educational-games/ click-if-you-agree-grades-7-9. Accessed: 2016-08-13.

[45] MediaSmarts. Co-Co's AdverSmarts: An Interactive Unit on Food Marketing on the Web. http://mediasmarts.ca/game/ co-cos-adversmarts-interactive-unit-food-marketing-web. Accessed: 2016-08-13.

[46] MediaSmarts. CyberSense and Nonsense: The Second Adventure of The Three CyberPigs. http://mediasmarts.ca/game/ cybersense-and-nonsense-second-adventure-three-cyberpigs. Accessed: 2016-06-11.

[47] MediaSmarts. Jo Cool or Jo Fool, Cyber Tour with Josie and Joseph Cool. http://mediasmarts.ca/sites/mediasmarts/files/games/joecool_ joefool/en/start_tour.html. Accessed: 2016-06-19.

[48] MediaSmarts. Jo Cool or Jo Fool, Teacher's Guide. http://mediasmarts.ca/ sites/mediasmarts/files/guides/jo_teachers_guide.pdf. Accessed: 201607-9. 
[49] MediaSmarts. MediaSmarts Educational Games. http://mediasmarts.ca/ digital-media-literacy/educational-games. Accessed: 2016-08-13.

[50] MediaSmarts. Privacy Pirates: An Interactive Unit on Online Privacy (Ages 7-9). http://mediasmarts.ca/game/ privacy-pirates-interactive-unit-online-privacy-ages-7-9. Accessed: 2016-08-13.

[51] MediaSmarts. Privacy Playground: The First Adventure of the Three Cyberpigs. http://mediasmarts.ca/game/ privacy-playground-first-adventure-three-cyberpigs. Accessed: 2016-06-11.

[52] L. Medina. Lorelyn Medina Illustrations. http://www. shutterstock.com/g/ Lorelyn+Medina. Accessed: 2016-08-01.

[53] Merriam Webster. Merriam Webster's definition of Cyberbullying for students. http://www.merriam-webster.com/dictionary/cyberbullying. Accessed: 2016-07-01.

[54] J. C. Meyer. Humor as a Double-Edged Sword: Four Functions of Humor in Communication. Communication Theory, 10(3):310-331, 2000.

[55] Microsoft. Microsoft Secure. https://www.microsoft.com/en-us/security/ default.aspx. Accessed: 2016-06-11.

[56] Mojang Synergies AB. Mine Craft Creators of the Temple Run series. https://minecraft.net/en/.

[57] A. Mulholland. www.ctvnews.ca Minecraft: Why Are Kids, and Educators, So Crazy For It? http://www.ctvnews.ca/lifestyle/ minecraft-why-are-kids-and-educators-so-crazy-for-it-1.2006975, September 2014. Accessed: 2016-08-17.

[58] R. Munroe. XKCD: A Webcomic of Romance, Sarcasm, Math, and Language. http://xkcd.com/936/. Accessed: 2016-06-11.

[59] A. Negrete and C. Lartigue. Learning from education to communicate science as a good story. Endeavour, 28(3):120124, September 2004.

[60] Office of Privacy Commissioner of Canada. Social Smarts: Privacy, the Internet and You. https://www.priv.gc.ca/youth-jeunes/fs-fi/res/gn_index_e. asp. Accessed: 2016-06-11.

[61] Orkidstra. Orkidstra program by the Leading Note Foundation. http:// leadingnotefoundation.org/. Accessed: 2016-07-16. 
[62] D. L. Paulhus. Measurement and control of response bias, pages 17-59. Academic Press, San Diego, CA, US, 1991.

[63] Paypal. Buyer Protection. https://www.paypal.com/ca/webapps/mpp/ security/buyer-protection. Accessed: 2016-06-11.

[64] D. Peters. Interface Design for Learning: Design Strategies for Learning Experiences. New Riders, 30 Corporate Drive, Suite 400, Burlington, MA 01803, 12 2013.

[65] J. Piaget. Science of Education and the Psychology of the Child. Orion Press, New York, USA, 1970.

[66] J. Poppenk, S. Khler, and M. Moscovitch. Revisiting the novelty effect: When familiarity, not novelty, enhances memory. Journal of Experimental Psychology: Learning, Memory, and Cognition, 36(5):1321-1330, 2010.

[67] Purdue University. online.purdue.edu The Evolution of Technology in the Classroom. http://online.purdue.edu/ldt/learning-design-technology/ resources/evolution-technology-classroom. Accessed: 2016-08-15.

[68] L. Rainie. 13 Things to Know About Teens and Technology. http://www. pewinternet.org/2014/07/23/ 13-things-to-know-about-teens-and-technology/. Accessed: 2016-06-11.

[69] K. Raynes-Goldie. Gaming Privacy : a Canadian case study of a children's cocreated privacy literacy game. In Surveillance 83 Society, volume Vol 12, No 3, pages 414-426, New York, NY, USA, Sept 2014. Surveillance Studies Network, Queens University.

[70] A. Repenning and C. Lewis. Playing a Game: The Ecology of Designing, Building and Testing Games as Educational Activities. In Piet Kommers and Griff Richards, editors, Proceedings of EdMedia: World Conference on Educational Media and Technology 2005, pages 4901-4905, Montreal, Canada, June 2005. Association for the Advancement of Computing in Education (AACE).

[71] I. Rivers and N. Noret. I h8 u: findings from a five-year study of text and email bullying. British Educational Research Journal, 36(4):643-671, 2010.

[72] E. Rubegni and M. Landoni. Fiabot!: Design and Evaluation of a Mobile Storytelling Application for Schools. In Proceedings of the 2014 Conference on Interaction Design and Children, IDC '14, pages 165-174, New York, NY, USA, 2014. ACM.

[73] R. M. Ryan and E. L. Deci. Intrinsic and extrinsic motivations: Classic definitions and new directions. Contemporary Educational Psychology, pages 54-67, 2000. 
[74] N. S. Said. An Engaging Multimedia Design Model. In Proceedings of the 2004 Conference on Interaction Design and Children: Building a Community, IDC '04, pages 169-172, New York, NY, USA, 2004. ACM.

[75] K. Sangani and M. A. Sasse. BOYD to the Classroom. Engineering and Technology, pages 42-45, April 2013.

[76] M. Sharples, R. Garber, C. Harrison, and K. Logan. E-safety and Web 2.0 for children aged 11-16. In Proceedings of the Journal of Computer Assisted Learning, 2009.

[77] S. Sheng, B. Magnien, P. Kumaraguru, A. Acquisti, L. F. Cranor, J. Hong, and E. Nunge. Anti-Phishing Phil: The Design and Evaluation of a Game That Teaches People Not to Fall for Phish. In Proceedings of the 3rd Symposium on Usable Privacy and Security, SOUPS '07, pages 88-99, New York, NY, USA, 2007. ACM.

[78] S. Srikwan and M. Jakobsson. Using Cartoons to Teach Internet Security. Cryptologia, 32(2):137-154, April 2008.

[79] V. Steeves. Summary of Research on Youth Online Privacy. Technical report, Office of the Privacy Commissioner of Canada, 2010.

[80] V. Steeves. Young Canadians in a Wired World, Phase iii: Life Online. Technical report, MediaSmarts, 2014.

[81] J. Svahn. The Everyday Practice of School Bullying. Children's participation in peer group activities and school-based anti-bullying initiatives, 2012.

[82] Tumblr. Tumblr Persona blogs. https://www.tumblr.com/. Accessed: 201606-11.

[83] L. van Wel and L. Royakkers. Ethical issues in web data mining. Ethics and Information Technology, 6(2):129-140, 2004.

[84] S. Vihavainen, T. Kuula, and M. Federley. Cross-use of Smart Phones and Printed Books in Primary School Education. In Proceedings of the 12th International Conference on Human Computer Interaction with Mobile Devices and Services, MobileHCI '10, pages 279-282, New York, NY, USA, 2010. ACM.

[85] A. Whitten and J. D. Tygar. Why Johnny Can't Encrypt: A Usability Evaluation of PGP 5.0. In Proceedings of the 8th Conference on USENIX Security Symposium - Volume 8, SSYM'99, pages 14-14, Berkeley, CA, USA, 1999. USENIX Association.

[86] Gamification Wiki. Gamification. https://badgeville.com/wiki/. Accessed: 201607-08. 
[87] L. Zhang-Kennedy and S. Chiasson. Teaching with an Interactive E-book to Improve Children's Online Privacy Knowledge. In Proceedings of the The 15th International Conference on Interaction Design and Children, IDC '16, pages 506-511, New York, NY, USA, 2016. ACM.

[88] L. Zhang-Kennedy, S. Chiasson, and R. Biddle. Password Advice Shouldn't Be Boring: Visualizing Password Guessing Attacks. In Anti-Phishing Working Group (APWG) eCrime Researchers Summit, pages 1-11. IEEE, 2013.

[89] L. Zhang-Kennedy, S. Chiasson, and R. Biddle. Password Advice Shouldn't Be Boring: Visualizing Password Guessing Attacks. In eCrime Researchers Summit (eCRS), 2013, pages 1-11, Sept 2013. 


\section{Appendix A}

\section{Appendix}

\section{A.1 JCJF detailed game scenarios}

According to the teacher's guide, the 12 scenarios can be classified into four main topics.

1. Online Marketing, E-Commerce and Data Collection

- Scenario 1: Splurge Cola. The scenario involves signing up to access fun content on the Splurge Cola website. Information gathered during sign up include name, age, sex, address, and phone number, in addition to giving seven friends' email addresses. Players have to decide whether it is "cool" or "fool" for Josie to share all this information about herself and her friends' emails. Once players select an option among these possible actions, the game displays an on-screen message describing the appropriate choice. Figure 3.1c-f shows the scenario screens.

- Scenario 2: CDRama. Jo is about to make a purchase online from the $C D$ Rama site. The scenario offers advice on how to notice reassuring signs when making a purchase online. In this scenario, the site is safe and the "cool" option is to pay through the site. A concluding message points out the different clues that should be used when deciding whether to trust an e-commerce site. The message also comments on whether Jo's choice was a cool or a fool action. Figure 3.2a shows the opening scene of the scenario.

- Scenario 6: Lotsa Music. The site offers enticing prizes as part of a contest. Jo needs to submit an answer to a skills testing question by filling a form that requests his full name, age, address, phone number, and email address. A message with the lesson behind the scenario appears once the player decides whether it is "cool" or "fool" to submit a form for this contest. 
Figure 3.2e shows the second page of the scenario collecting way too much personal information.

2. Online Safety

- Scenario 3: Fast Talk. Fast Talk is an online chatting website where Josie can meet people online. To increase her chance of finding the right mate, Josie is considering revealing her home address to ensure the relative closeness of her future boyfriend. The message at the end of the scenario points out the risk behind revealing such information. Figure $3.2 \mathrm{~b}$ shows the second page of the scenario offering the different types of chat, which ranges from romance to general chats.

- Scenario 10: My Home Page. Jo created a home page about himself as part of his homework assignment. Jo revealed too much personal information, by placing his image, school, street name, places where he can be found, and contact info. The final page of the scenario previews his teacher's feedback on Jo's mistakes and items he got right on the assignment. Jo's teacher pointed out how Jo revealed too much personal information, and how this might jeopardize his privacy and security both online and offline. Figure 3.2j-1 shows the opening scene and the third and last page of the scenario.

3. Authenticating Online Information

- Scenario 4: Homework Nook. While Jo worked on his homework assignment on "human rights," he came across the Homework Nook. The site turned out to be a hate site that advocated white supremacist views and how the Canadian Human Rights Act was to be blamed for taking jobs from white people. The final outcome is that Jo needed to be more careful of where he does his online research because sites with malicious motives can mask themselves as good ones. Figure 3.2c shows the opening scene of this scenario.

- Scenario 5: Teen World. Teen World is online magazine that Josie found. 
They offer tips on various topics. The most enticing is how to hack a computer's firewall to access the results of a math test. Josie has to register on the site to access most of its content. Josie thinks that she should pass because she does not trust the site's publishers. The last page of the scenario points out that Josie made the right decision and explains how hacking is illegal in Canada. Figure 3.2d shows the teenworld.com site with the "Hackers Tips".

- Scenario 7: Summer Daze Tour. Josie visits Summer Daze Tour and finds a rock concert coming up. She is ready to pay for the ticket with her hard earned money, and join some friends in a van to attend the Californiabased concert. The site promises to send the tickets by mail once Josie mails a cheque or money order to the address on the site. Josie learns the hard lesson of needing to investigate before sending any money to an unknown address. Figure $3.2 \mathrm{f}$ shows the second page view of the scenario.

- Scenario 9: Cheap Mail. Cheapmail deals with the issue of hacking into someone else's email address. Josie is asked to reply to a hacking email by providing the email address of the person she wishes to hack, along with her Cheapmail password. The site promises that Josie will soon receive the person's email password. Josie ends up making the right choice by trashing this email. The final message for this scenario is to trust your instinct if something sounds too good to be true. Figure 3.2i shows the opening scene this scenario.

\section{Responsible Internet Use}

- Scenario 8: ICU-UCME. The instant messaging service $I C U-U C M E$ offers online chatting. Jo notices a message between his younger brother Jay and a friend, where Jay was really disrespectful to his friend. Jay said that he was only joking but his friend took it seriously. The lesson behind the scenario is for Jay to mind how he treats others even if they are online, and not to forget his morals because the conversation is not face-to-face. Figure 3.2g-h shows the opening scene and the second page of the scenario. 
- Scenario 11: Voting Booth. Voting Booth is a online webpage where you can create a custom vote on any topic. Josie decided to have her friends rate her school's teachers. Josie ended up in trouble because she could not control her friends' votes. Some wrote really mean comments along with their votes. The lesson behind this scenario is that if you wish to create a public vote, and to understand the consequences of your actions. Figure $3.2 \mathrm{~m}$ shows the second page of the scenario, which represents the different vote options.

- Scenario 12: Essay World. Jo has an English essay to write and it is due in two days. He is thinking of using the Essay World site for help. It offers free essays already written. His friend Jason is going to use it. Jo ends up not using this site and writing his own essay. Jo's teacher finds out about his friend Jason's plagiarism through a quick web search and Jo receives the better mark. Figure 3.2n shows the opening scene of the scenario.

The game scenarios are structured so the player can go through them in chronological order or choose the particular topic individually Figure 3.1b shows the main page with links to the games scenarios). 
A.2 Parental/Legal Guardian Permission for Children Participation in Research 


\section{Carleton \\ U N I V E R S I T Y \\ Canada's Capital University}

Parental/Legal Guardian Permission for Children Participation in Research

Title of research project: Evaluating a privacy game intended for children

Date of ethics clearance: June 03, 2015

Ethics Clearance for the Collection of Data Expires: May 31, 2016

\section{Introduction}

The purpose of this form is to provide you (as the parent/legal guardian of a prospective research study participant) information that may affect your decision as to whether or not to let your child participate in this research study. The person performing the research will describe the study to you and answer all your questions. Read the information below and ask any questions you might have before deciding whether or not to give your permission for your child to take part. If you decide to let your child be involved in this study, this form will be used to record your permission.

\section{Purpose of the Study}

If you agree, your child will be asked to participate in a research study evaluating a computer game to teach about online privacy. This research is being conducted in collaboration with MediaSmarts, a not-for-profit organization. The results of the study will be used to determine how effective the new design of the game.

What is my child going to be asked to do?

Your child will be asked to play a short computer game. This is in order to gauge the effectiveness of the new game "A Day in the Life of The JOs" in educating the children ages 11 to 12 about the different risks of sharing information online. The user study will be audio-recorded, also the computer interactions will be recorded using an eyetracking device. This study will take approximately 30 minutes. There are no foreseeable risks to participating in this study. Your child will not be asked to disclose any personally identifiable information. However if such information is voluntarily disclosed, it will be discarded when transcribing the interview to text. The possible benefit of participation is for your child to know more about the risks of sharing too much private information on the different online sites.

Does my child have to participate?

No, your child's participation in this study is voluntary. Your child may decline to participate or to withdraw from participation at any time.

What if my child does not want to participate?

In addition to your permission, your child must agree to participate in the study. If you child does not want to participate they will not be included in the study and there will be no penalty. Even if your child initially agrees to be in the study, they can change their mind later without any penalty.

Your child will receive a $\$ 20$ gift card to Chapters for participating in this study.

How will your child's privacy and confidentiality be protected if $s /$ he participates in this research study?

Figure A.1: Parental / Legal Guardian Permission for Children Participation in Research page 1. 
Your child's privacy and the confidentiality of his/her data will be protected by storing audio- and the eye-tracking-recordings electronically on a password-protected computer. Access will be restricted to those researchers directly involved with the research. The anonymized data and associated analysis will be stored on a passwordprotected computer. If we become aware that the participant is engaging in dangerous actions online, we will disclose this information to the REB.

The only personally identifiable information will be the signed paper consent forms. These will be kept in a locked filing cabinet and not associated with the electronic data.

This project was reviewed and received ethics clearance by the Carleton University Research Ethics Board (REB). Contact information for the REB Chair and Vice-Chair follows:

Professor Louise Heslop, Chair

Research Ethics Board

Carleton University Research Office

Carleton University

1125 Colonel By Drive

Ottawa, Ontario K1S 5B6

Tel: 613-520-2517

E-mail: ethics@carleton.ca

The principal researchers involved in this research are:

Christine Mekhail, MA. Student

School of Human-Computer Interactions

Carleton University

1125 Colonel By Drive

Ottawa, Ontario K1S 5B6

Tel:

Email: ChristineMekhail@cmail.carleton.ca
Sonia Chiasson, Assistant Professor School of Computer Science

Carleton University

1125 Colonel By Drive

Ottawa, Ontario K1S 5B6

Tel: (613) 520-2600 ext. 1656

Email: chiasson@scs.carleton.ca
Audio-recording is allowed
Eye-tracking is allowed
YES $\quad$ NO
YES NO
I, give permission to my child,
to participate in this study evaluating a computer

game teaching about online privacy

Signature of parent(s) or Legal Guardian

Signature of researcher
Date

Date

Figure A.2: Parental / Legal Guardian Permission for Children Participation in Research page 2 . 


\section{Carleton \\ Canada's Capital University}

\section{Verbal Informed Assent Script for the Children}

$\mathrm{Hi}$, my name is Christine Mekhail, a student at Carleton University. I need your help in testing this game called "A Day in The Life of The JOs.". I will ask you some questions and then play the game. Afterwards, I will ask you some more questions and then we're done.

This study will take about 30 minutes and I will record your voice while playing the game so that I can remember your comments. I will also use this eye-tracker to record where you're looking on the screen, so that I can refer back to them later.

The information you will share with me will be kept safely locked up. Nobody will know who said it except the people doing the research. Just remember we are testing the game and not you. Nothing you will say today will hurt our feelings. However, if you tell us about dangerous behaviour, we will tell our Research Ethics Board so that someone can make sure that you are safe.

You do not have to join this study. It is up to you. You can say okay now and change your mind later. All you have to do is tell me that you want to stop. No one will be mad at you. Just let me know.

To thank you for your help, I will give you a $\$ 20$ gift card from Chapters. You can keep the gift card even if you decide to stop the study.

Do you have any questions? Remember that you can ask questions at any time.

Do you agree in participating in this user study and playing the game?

If yes, please say: yes, I will be in this research study

If no, please say: no, I don't want to do this

Figure A.3: Verbal Informed Assent Script for the Children

\section{A.3 Verbal Informed Assent Script for the Children}


A.4 Debriefing Form 


\section{Carleton \\ U N I V E R S I T Y \\ Canada's Capital University}

\section{Debriefing Form}

The research we are conducting helps us evaluate the effectiveness of the privacy game "A Day in The Life of The JOs" in educating children of the age group 11 to 12 about safe online interaction when it comes to sharing information.

The results of this study will be used to identify areas where the game can be updated or changed in order to improve effectiveness in changing the children's behavior. Your thoughts, comments, and opinions are very helpful and valuable to our research. Thank you for participating in this study. Your time and effort are greatly appreciated!

If you have any further questions regarding this research, please contact:

Christine Mekhail, MA. Student

School of Human-Computer Interaction

Carleton University

1125 Colonel By Drive

Ottawa, Ontario K1S 5B6

Tel:

Email: ChristineMekhail@cmail.carleton.ca
Sonia Chiasson, Assistant Professor School of Computer Science

Carleton University

1125 Colonel By Drive

Ottawa, Ontario K1S 5B6

Tel: (613) 520-2600 ext. 1656

Email: chiasson@scs.carleton.ca

If you have any concerns about the ethics of this research, please contact:

Professor Louise Heslop, Chair

Research Ethics Board

Carleton University Research Office

Carleton University

1125 Colonel By Drive

Ottawa, Ontario K1S 5B6

Tel: 613-520-2517

E-mail: ethics@carleton.ca

Figure A.4: Debriefing Form 
A.5 User Feedback Questionnaire 


\section{The post-test interview:}

1. The content was...

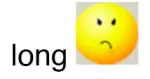

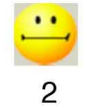
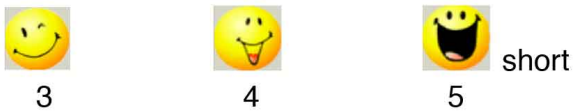

2. The game scenarios were ...
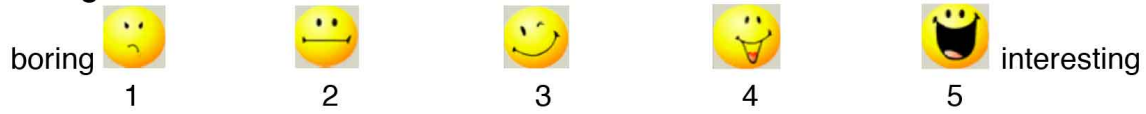

3. Playing the game was...
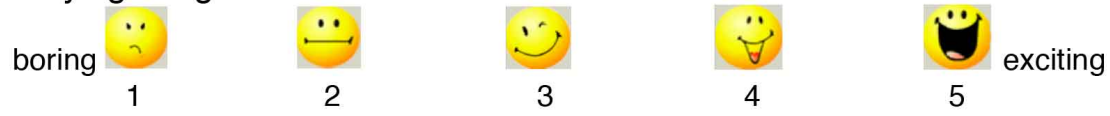

4. How much did you learn?
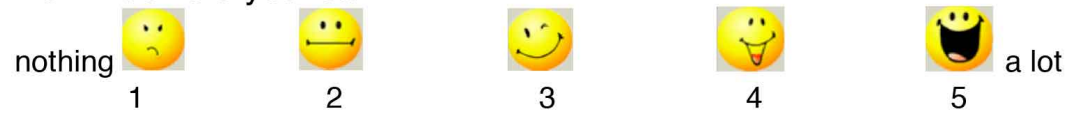

5. The game was...
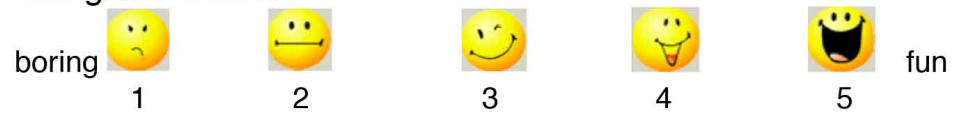

6. The game design was...
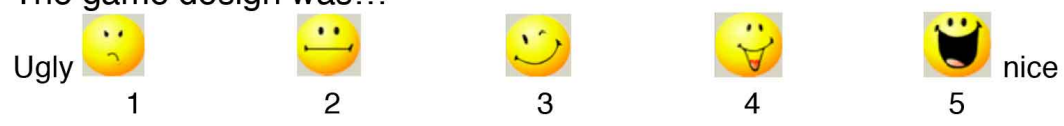

7. Would you recommend this game to a friend?
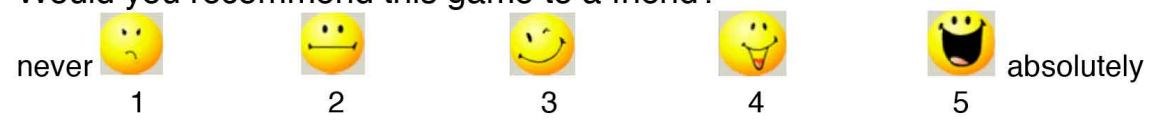

Figure A.5: User Feedback Questionnaire 


\section{A.6 Pre- and Post-game-day Interviews}

\section{D1 - cyberbullying:}

1. What is cyberbullying?

2. If you saw someone being bullied online, what can you do to help that person?

\section{D2 - data mining:}

1. What information about yourself is okay to share with other websites if they asked you to?

2. Do you know what a website cookie is?

D3 - privacy:

1. What is online privacy?

2. How can you protect your privacy online? What are some of the ways to do so?

\section{D4 - photo sharing:}

1. Is it okay to share images of your friends? Do you need to do anything first?

2. How do you decide if you can use a picture that you found online?

\section{D5 - verification of information from online sources:}

1. How can you tell if information you find online is true?

2. If you've seen a post on Instagram that your favourite store in the mall is giving away $\$ 50$ gift cards, what would you do? 


\section{A.7 D1-Cyberbullyng Day full script:}

9:08 am A little after first class started:

GRAPHIC: Text message on Josie's phone

SOFIA OMG did you see how Cindy was staring at Brian in band today? CRUSHING SO HARD

GAME TEXT: What should Josie do?

1. That's great news! Josie should post on Cindy's wall: Brian's perfect for you! Great to hear that C ¡3's B! 2. Hmm... Josie should text to Sofia: R U sure Cindy wants people to know about this? She might not be happy if it gets to Brian before she can talk 2 him. 3. This is too good not to share! Josie should text to Jo: Did U hear about Cindy and Brian? Keep quiet about it though

1. Post on Cindy's wall. (0 points) Social Media post on Josie's wall:

CINDY: Mind UR own business JOSIE! Brian read your post \& all his friends R laughing now

CINDY has unfriended you.

2. Text the person who sent her the news. (2 points) Text from Sofia: OMG I didn't think of that! I checked w Cindy \& she was totally embarrassed so don't tell any1 plz! Thanks for checking first!

3. Text Jo. (1 point) Social media post on Jo's wall:

JO: Hey BRIAN way 2 go! BRIAN: ??? JO: If U feel eyes on UR back? it's bcuz some1 has a crush on you! BRIAN: Who sits behind me? CINDY? JO: Aw man! don't tell CINDY or Jo I'm the one who told $u$, it was supposed to be a secret

11:10 am during lunch:

TEXT FROM JAY: Hey Jo check out this video I took in class last week!

GRAPHIC: Video-sharing site (still picture) showing a boy with marker on his face. The video's title is BEN FALLS ASLEEP IN CLASS. Below are the following COMMENTS:

JAY (original poster): Check out this video of Ben falling asleep in class and not even noticing when we WROTE IN MARKER ON HIS FACE! KOOLDUDE: LOL GREY51: What a luzr ROB: I'm sharing this with all my friends!

GAME TEXT: What should Jo do? 
1. This is too funny! Jo should post a comment: Sorry Ben but this is hilarious! 2. A joke's a joke, but this is too far. Jo should flag the video to report it to the video site and text BEN: Hey man JAY shouldn't have done that to u. 3. Ben's a friend. Jo should post a comment: Don't be a jerk Jay, take the video down before more ppl see it.

1. Post a funny comment of his own. (0 points) Comments on video:

JO: Sorry Ben but this is hilarious! BEN: Hey JO since U like funny videos so much here's one of U bellyflopping at camp last summer (http://vidwow.ca/jos-big-bellyflop) JAY: LOL BEN: Don't laugh JAY, UR next? (http://vidwow.ca/jay-in-a-tutu)

2. Report the video (2 points)

EMAIL from the video company: Thank you for your report. We have reviewed the video and found that it violates our terms of service.

TEXT from BEN: Thanks man, it feels better knowing I have some REAL friends.

3. Leave a comment (1 point)

Comments under the video:

JO: Don't be a jerk Jay, take the video down before more ppl see it. JAY: Chill dude, Ben's not complaining? GREY51: Yeah it's just a joke ROB: If UR so sensitive why don't U go play Cuddle Bears JAY: LOL!

2:26 pm towards the end of third period:

GRAPHIC: Closeup of a black-and-white yearbook photo of a teen boy with "Flock of Seagulls" hair. Underneath is the caption Grad JEFF SIMPKIN shows off his super-cool new look.

GAME TEXT: OMG, Jo was working on his school yearbook and found this old picture of Principal Simpkin. What should he do?

1. People have to see this RIGHT AWAY! He should post it to the school's online yearbook page. 2. Too funny not to share, but maybe not with everybody. He should send it to his friends for a laugh but not put it in the yearbook. 3. Maybe he should email the principal to make sure he finds it funny too.

1. Post it to the school's yearbook page. (0 points)

School yearbook page: It would be great if we could start with the photo then have it fade away; if that's not possible just have the photo. Either way there should 
be the following comments:

JO: Check out Mr. SIMPKIN as a grad! NATE: LOL too funny JAY: (thumbs up icon) MR. SIMPKIN: JO, we need to talk in my office please?

2. Decide not to include it in the yearbook, but send it to his friends for a laugh. (0 points)

TEXT FROM JO: Hey NATE check out Mr. SIMPKIN as a grad? but dont share it with any1 kay? TEXT FROM NATE: OK

(a fadeout or a few blank spaces)

TEXT FROM NATE: want to catch a movie JO? TEXT FROM JO: cant I have detention? SOME1 shared that pic of Mr. SIMPKIN!

3. Check with the principal to make sure he finds it funny as well. (1 point)

EMAIL FROM JO TO MR. SIMPKIN: Hey Mr. SIMPKIN is it OK if I run this photo in the yearbook? EMAIL FROM MR. SIMPKIN: Oh my gosh, where did you find that? We should get old pictures of all the teachers for a collage. Great idea, JO, and thanks for checking with me!

3:18 pm Final period of the day:

Social media group (like a Facebook group) titled FB HIGH FIGHTING BISON BASKETBALL

JO has joined the group. NATE: OK guys we need 2 review our errors b4 the next game JAY: Yeah like letting MATT play NATE: LOL ok but srsly MATT: ha ha BRIAN: srsly how do u miss a shot like that? JAY: New rule nerds never take the ball BRIAN: Yeah or losers or geeks or feebs? so MATT basically MATT: OK OK! lets talk about NEXT game

GAME TEXT: Jo just checked in with his basketball team online and saw these posts. What should he do? 1. They're really being cruel. He should stand up to it by posting to the group: Guys?. It's only one game. Remember all those other great shots MATT made. 2. Jo doesn't want to be a part of this. He should sign out before anyone notices he's there. 3. They're being really cruel, but it looks like Matt's trying to settle things down himself. Jo wants to do something to help, so he should send Matt a private message: Hey Matt, ignore those jerks. Everyone has a bad shot now and then! you'll do gr8 next game! 
1. Leave a comment (1 point) (in the group)

JO: Guys!. It's only one game. Remember all those other great shots MATT made. JAY: Come on JO we're just having fun NATE: JO's right, let's just focus on the next game JAY: Hey JO how about that time U tripped last game? BRIAN: HAHA LOL

2. Leave (0 points) (in the group)

JO has left the group. NATE: OK MATT just shape up b4 next game JAY: Good luck with that BRIAN: Yeah he just has to fix his jumping \& dribbling \& shooting! MATT has left the group. COACH BLAINE has joined the group. COACH BLAINE: Listen up guys, we don't do things like that here. You're ALL benched for the next game.

3. Talk to Matt later (2 points)

Private message from JO: Hey Matt, ignore those jerks. Every1 has a bad shot now and then! you'll do gr8 next game! Message from MATT: Thanks man! I didn’t want to let them see I was upset about it.

7:08 pm At home, later that night:

GRAPHIC: An online game a la Call of Duty of World of Warcraft (not animated) with an inset chat window.

Chat window:

BLUE LEADER: Hey JOSIE ready to get blasted off the map? JOSIE: I'll believe it when it happens! U guys R 2 games behind BLUE LEADER: Hey look u brought ur baby brother! is he going to cry when I blast $\mathrm{u}$ ? JOSIE: Why dont u stop talking \& play BLUE TEAM 2: Oh poor boo boo! does the baby need his mommy to fight for him?

GAME TEXT: What should Jo do?

1. Josie's the leader of her team. She should tell Blue Leader that acting like that isn't a part of the game and tell her team to mute and ignore him 2. She can't let someone insult Jo that way. She should say: Lay off my brother or I'll make U cry like a little baby! 3. She can't let someone insult her team that way. She should tell her team to show Blue Leader that they're better at trash-talking too.

1. Tell the player that acting like that isn't a part of the game and tell her team 
to mute and ignore him (2 points) Chat window: JOSIE: Blue Leader U R totally not worth it. Every1 mute him and lets just play. (it would be great if we could have BLUE LEADER's text turn grey or fade out.) JO: OK cool. SOFIA: what r ur orders JOSIE? JOSIE: OK lets start by taking out their plasma tank!

2. Step in and insult the player, for attacking her brother (0 points) Chat window: JOSIE: Lay off my brother or Ill make U cry like a little baby! BLUE LEADER: Yeah what $\mathrm{r}$ u going to do? throw his bottle at me? JOSIE: Im serious LAY OFF you $\$ \% \# \&$ JO: Hey sis cool down BLUE LEADER: hahaha? guess whos gonna be in trouble when I call the moderator

3. Tell her team to attack the player with equally mean words (0 points) Chat window:

JOSIE: OK guys, if they want a fight let's give them one SOFIA: What do u mean? JOSIE: Lets show em we can beat them at trash talk too. JO: Hey sis r u sure that's a good idea? The moderators might get mad JOSIE: That's an ORDER mister 


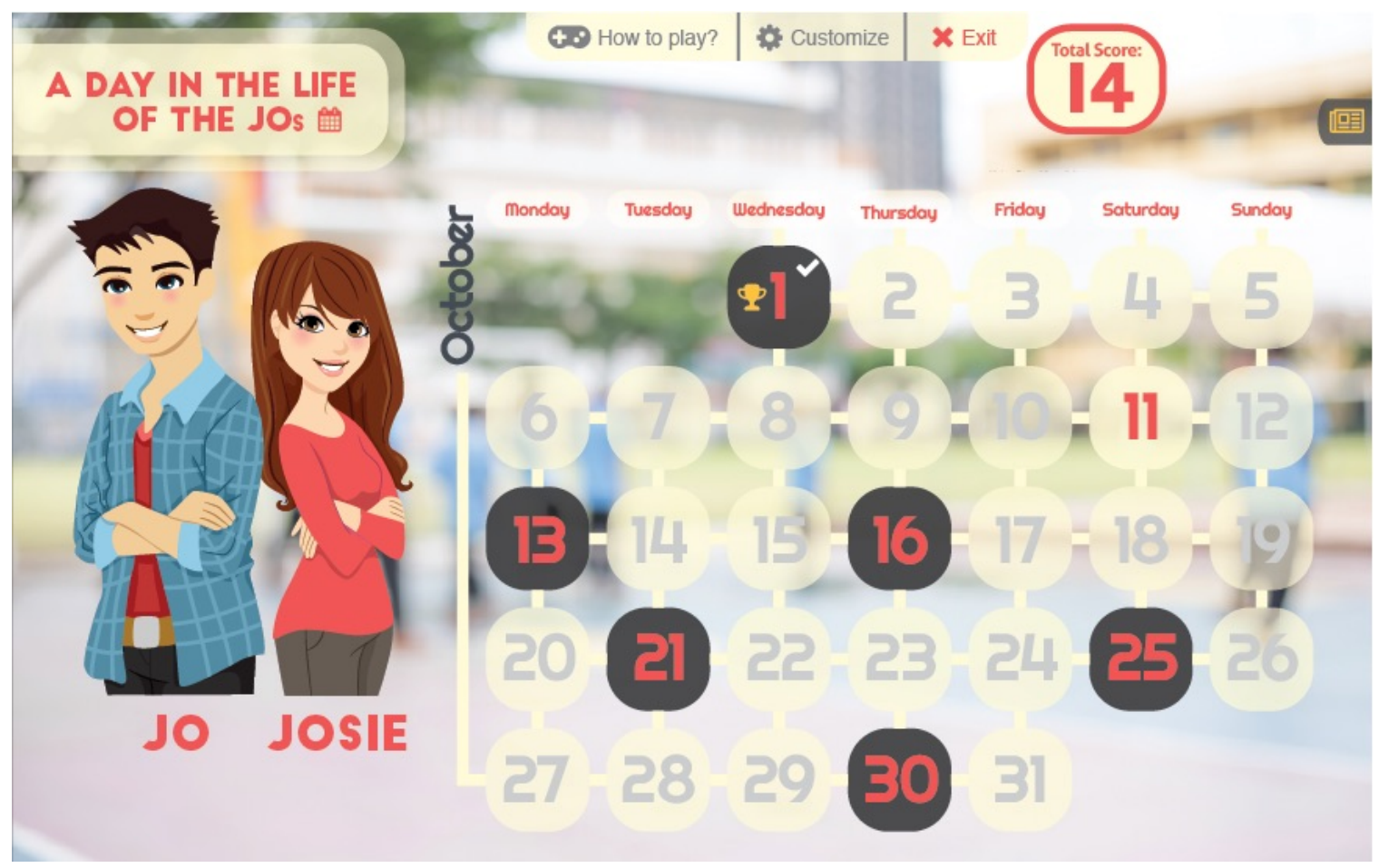

Figure A.6: The main landing page of ADITL. A calendar shows the active days containing scenarios in red.

\section{A.8 25 game scenarios across the five topics}

Below is a reference for the first three scenes of each of the 5 scenarios for the 5 days. 


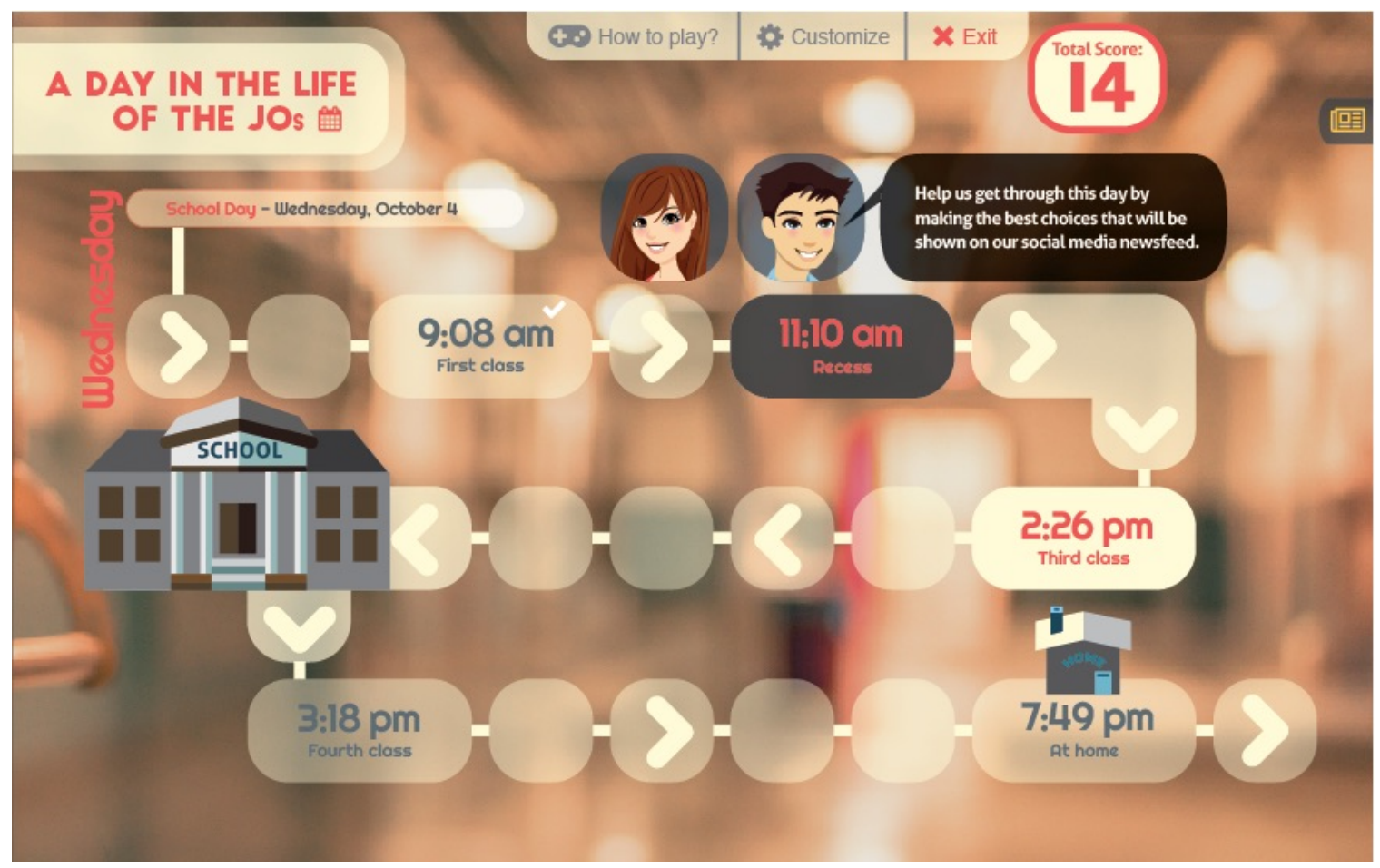

Figure A.7: The Day View hosts links to scenarios for a particular topic.

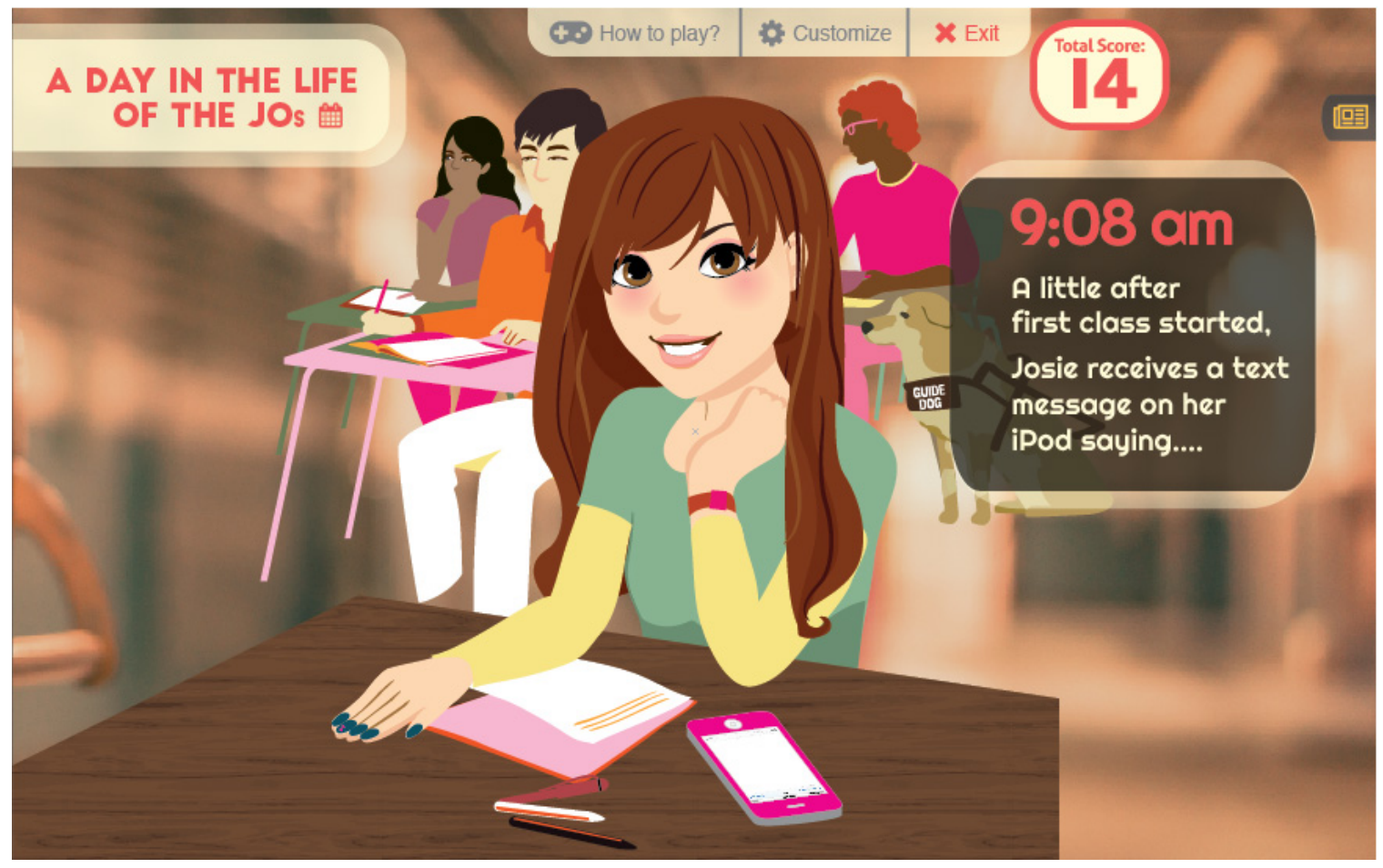

Figure A.8: D1-Cyberbullying: Scene 1 of first scenario. 


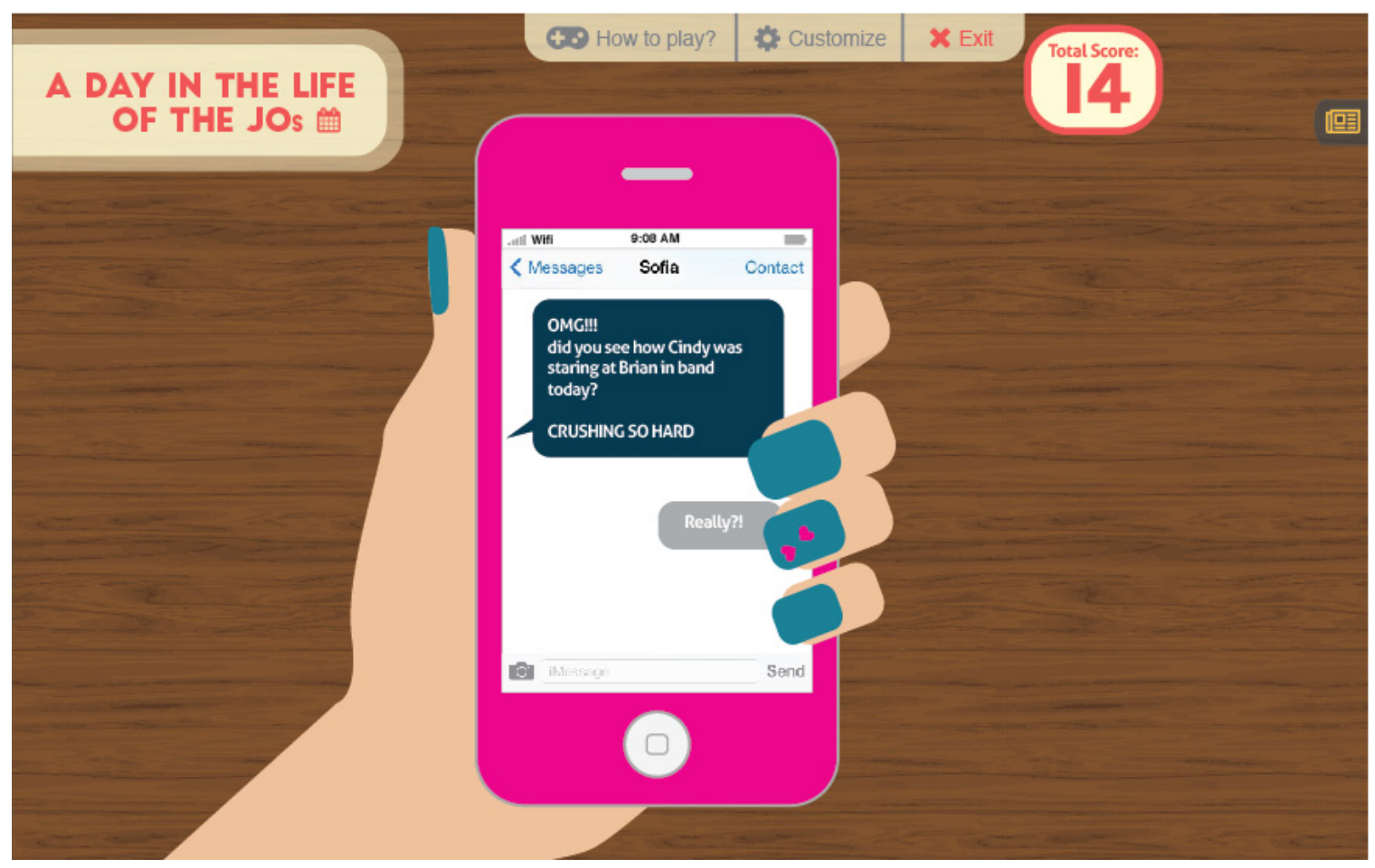

Figure A.9: D1-Cyberbullying: Scene 2 of first scenario.

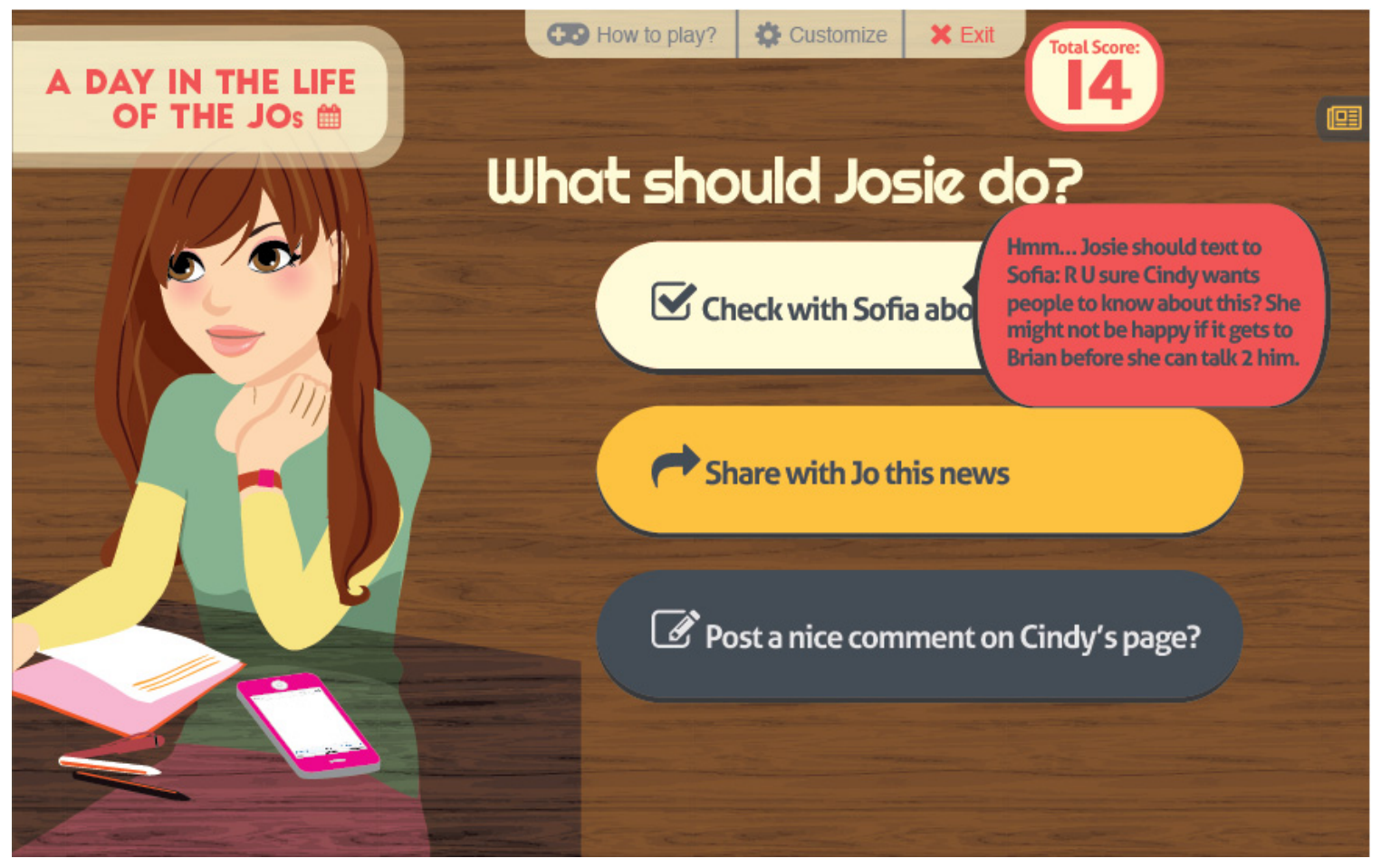

Figure A.10: D1-Cyberbullying: Scene 3 of first scenario. 


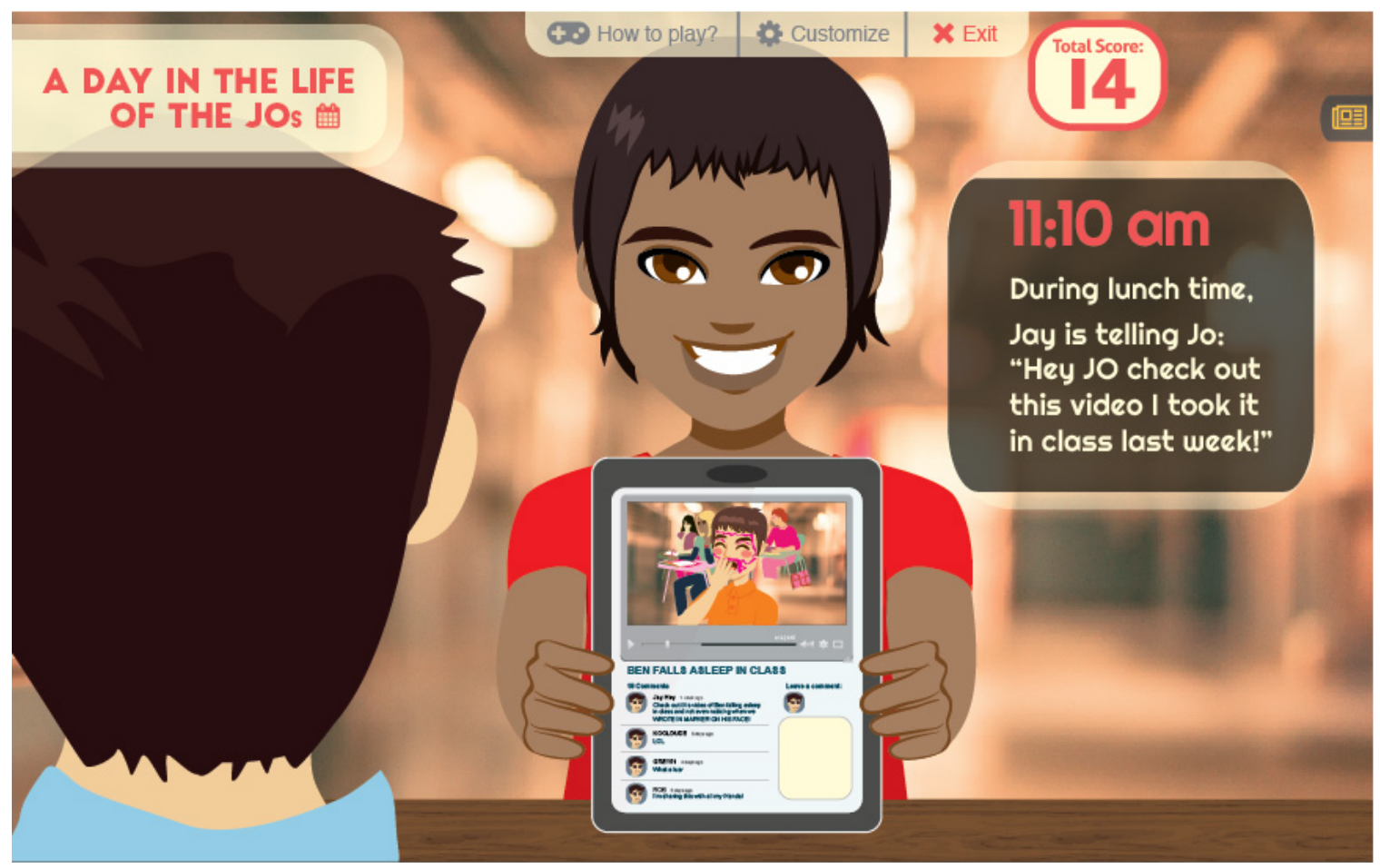

Figure A.11: D1-Cyberbullying: Scene 1 of second scenario.

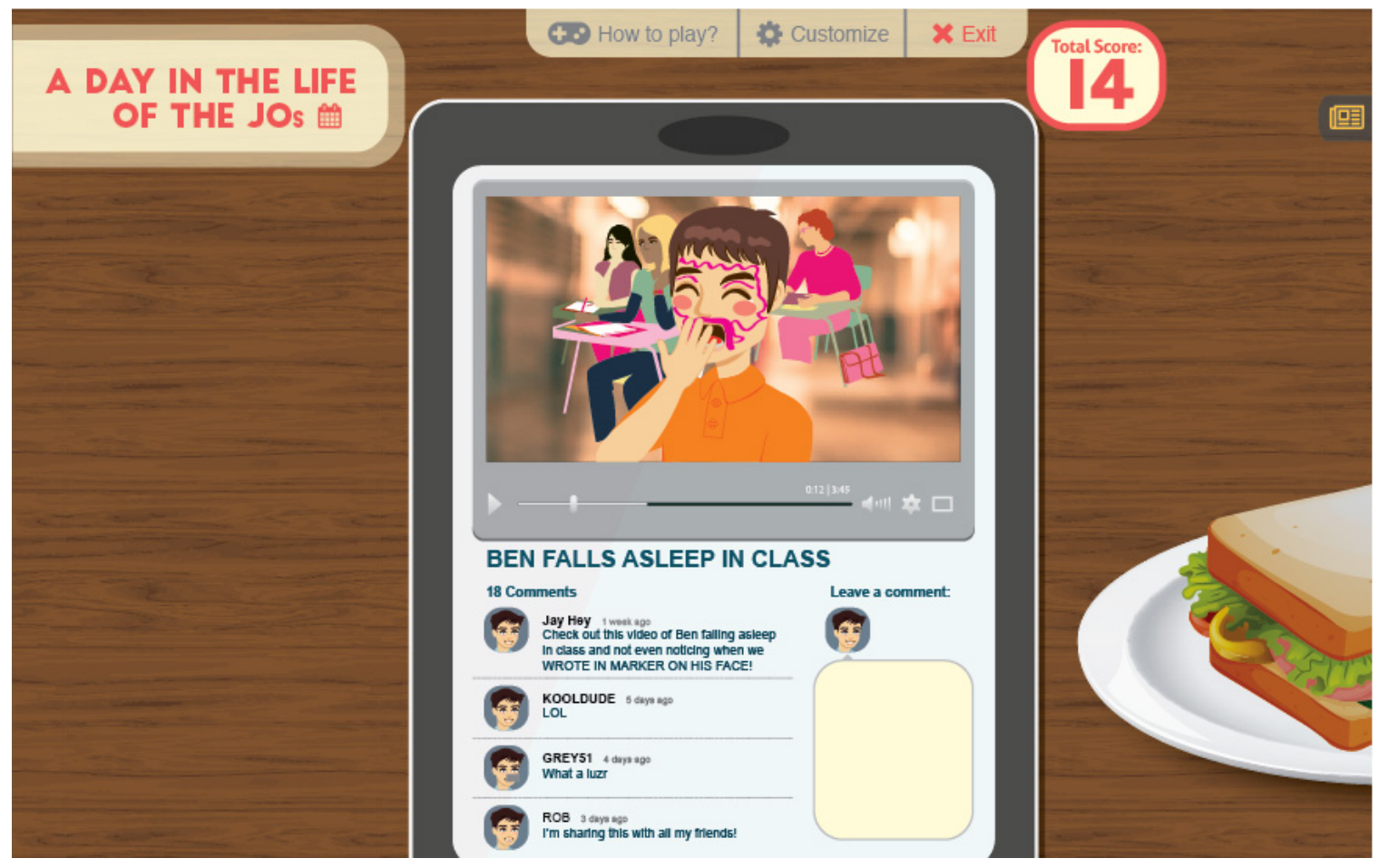

Figure A.12: D1-Cyberbullying: Scene 2 of second scenario. 


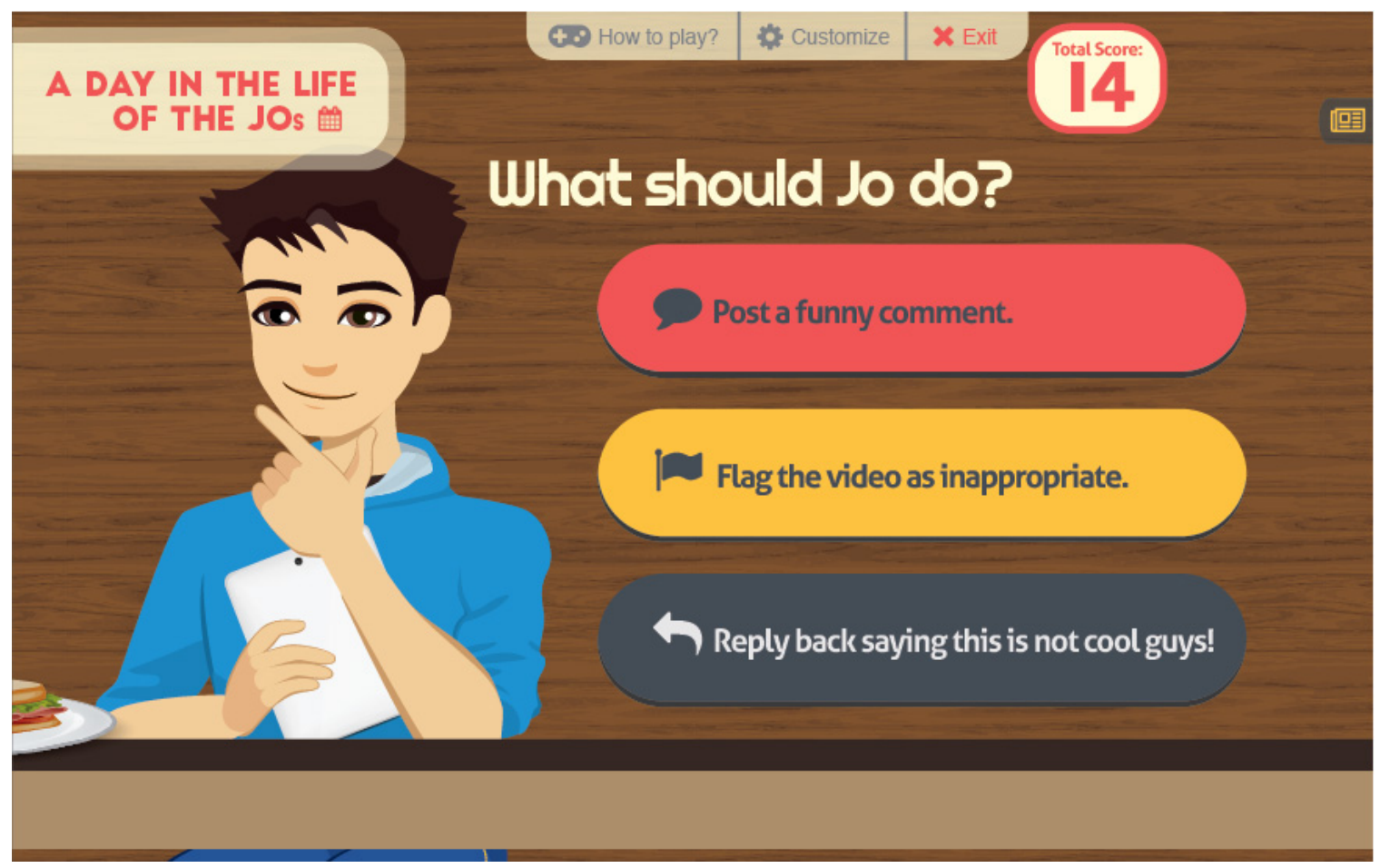

Figure A.13: D1-Cyberbullying: Scene 3 of second scenario.

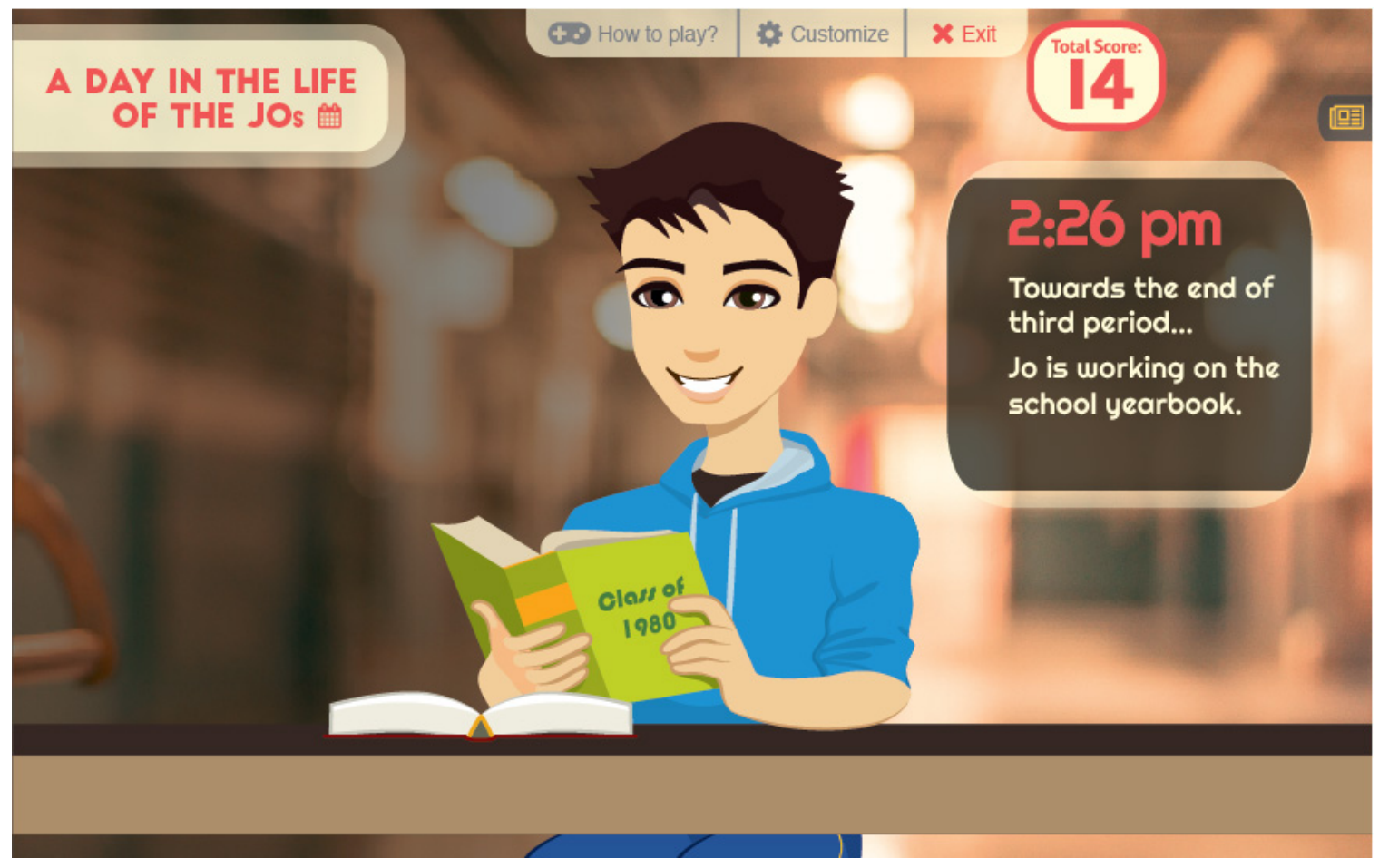

Figure A.14: D1-Cyberbullying: Scene 1 of third scenario. 


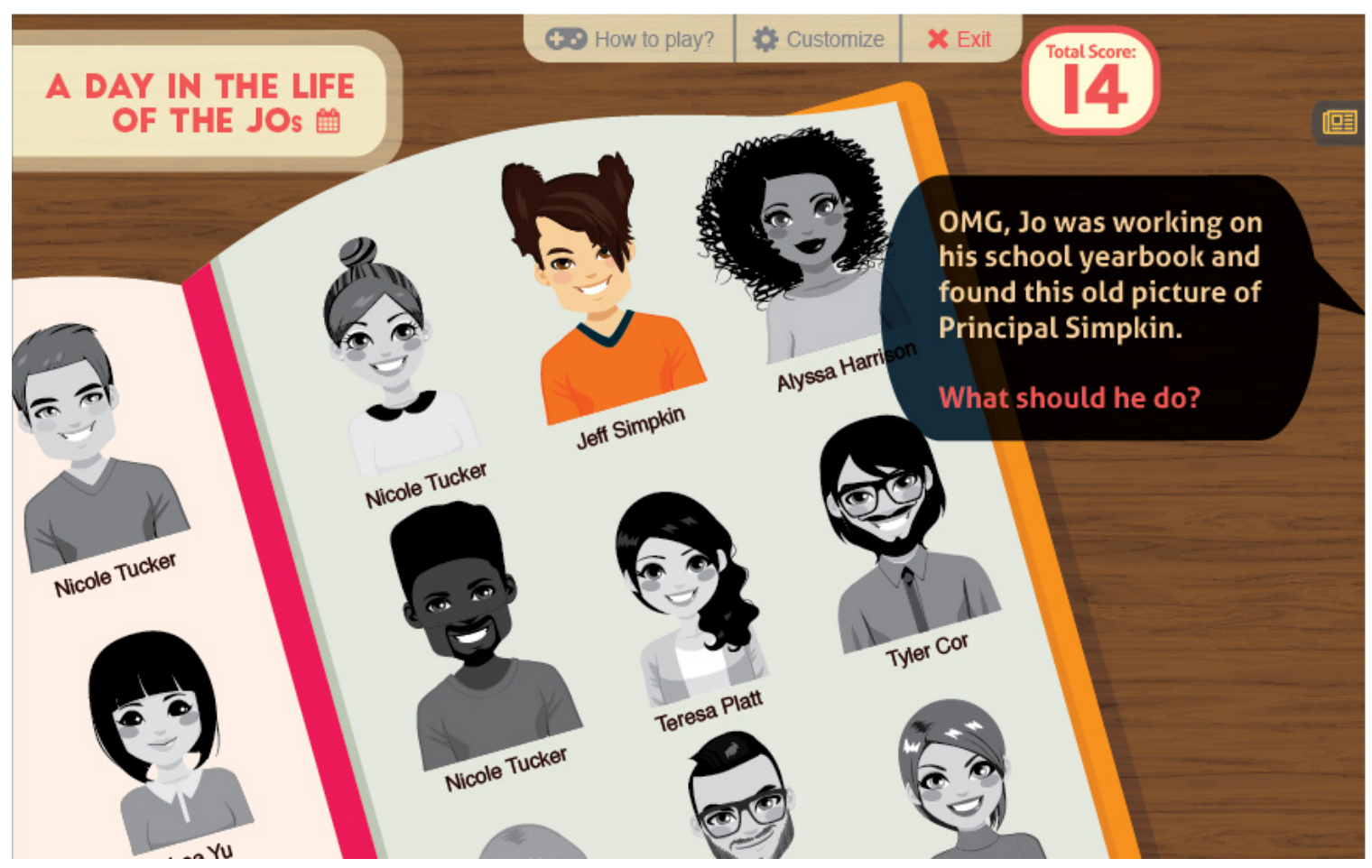

Figure A.15: D1-Cyberbullying: Scene 2 of third scenario.

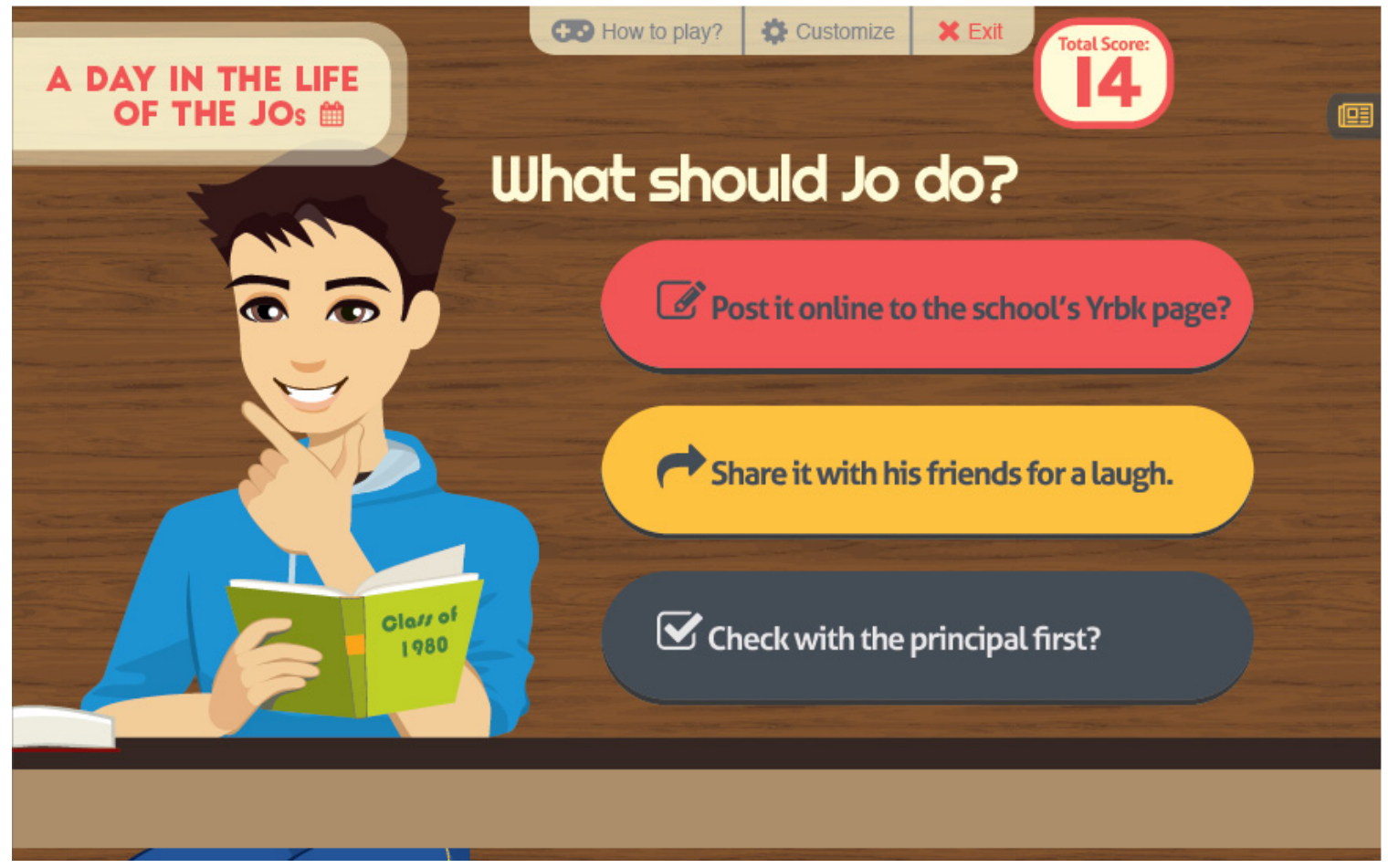

Figure A.16: D1-Cyberbullying: Scene 3 of third scenario. 


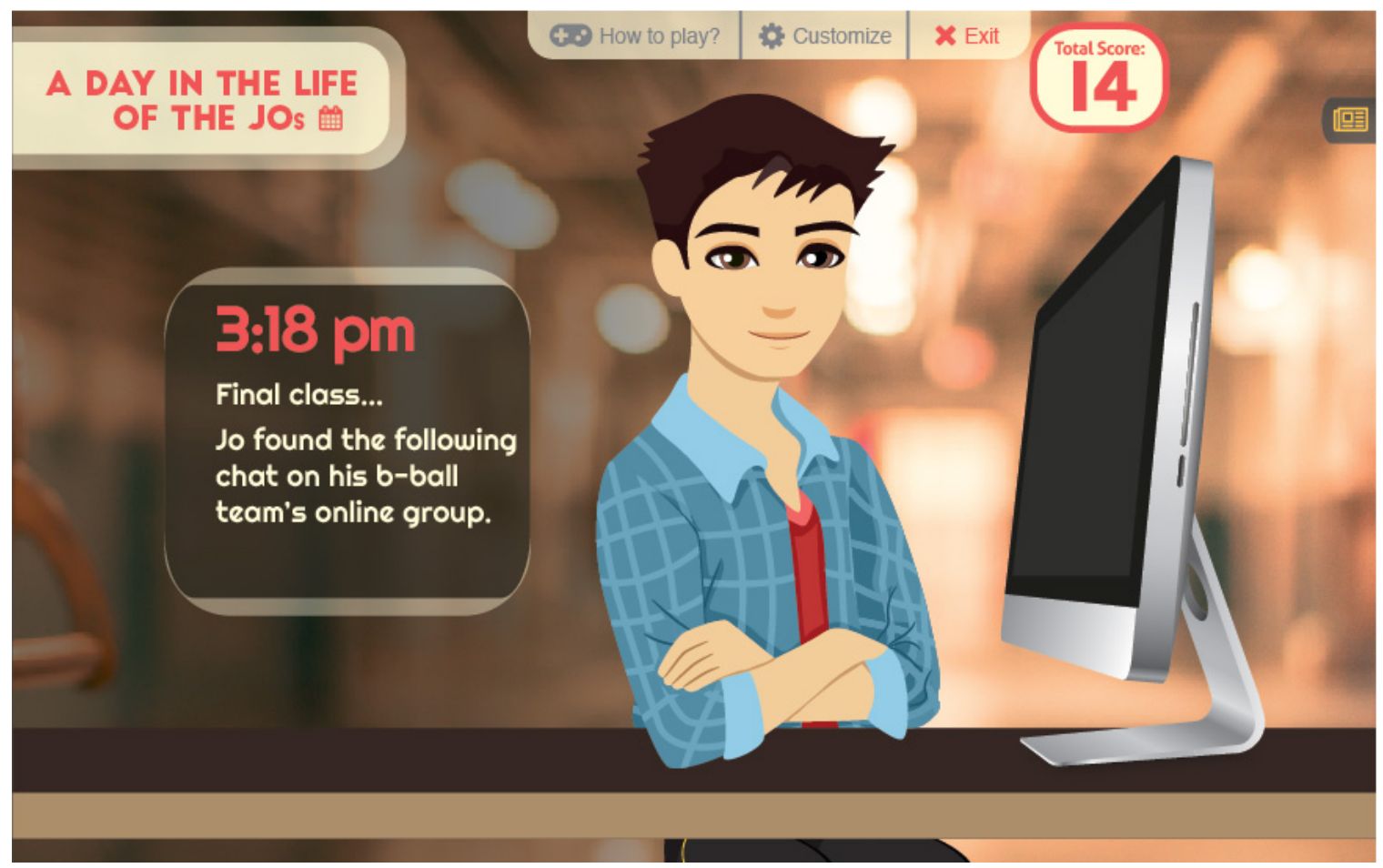

Figure A.17: D1-Cyberbullying: Scene 1 of fourth scenario.

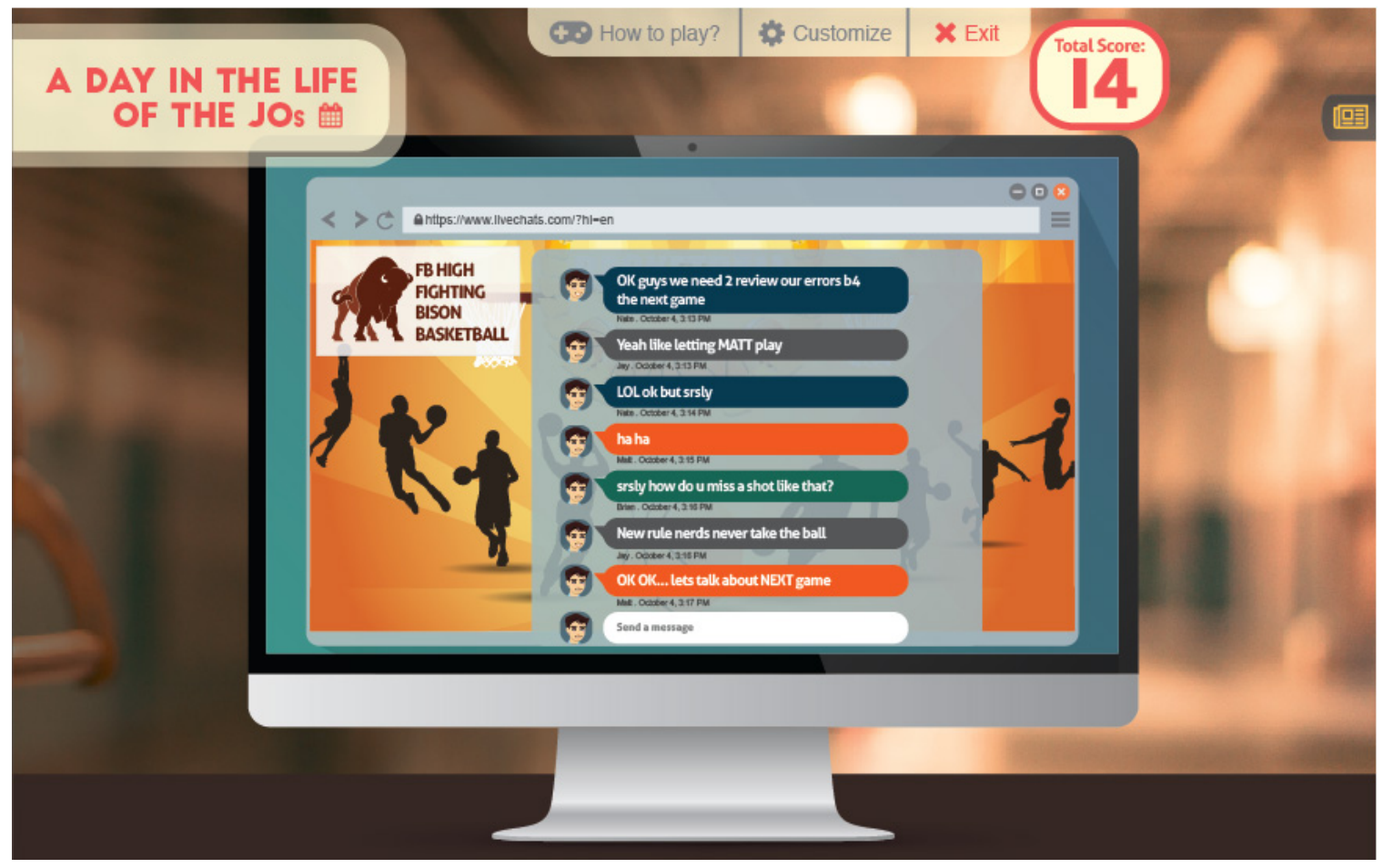

Figure A.18: D1-Cyberbullying: Scene 2 of fourth scenario. 


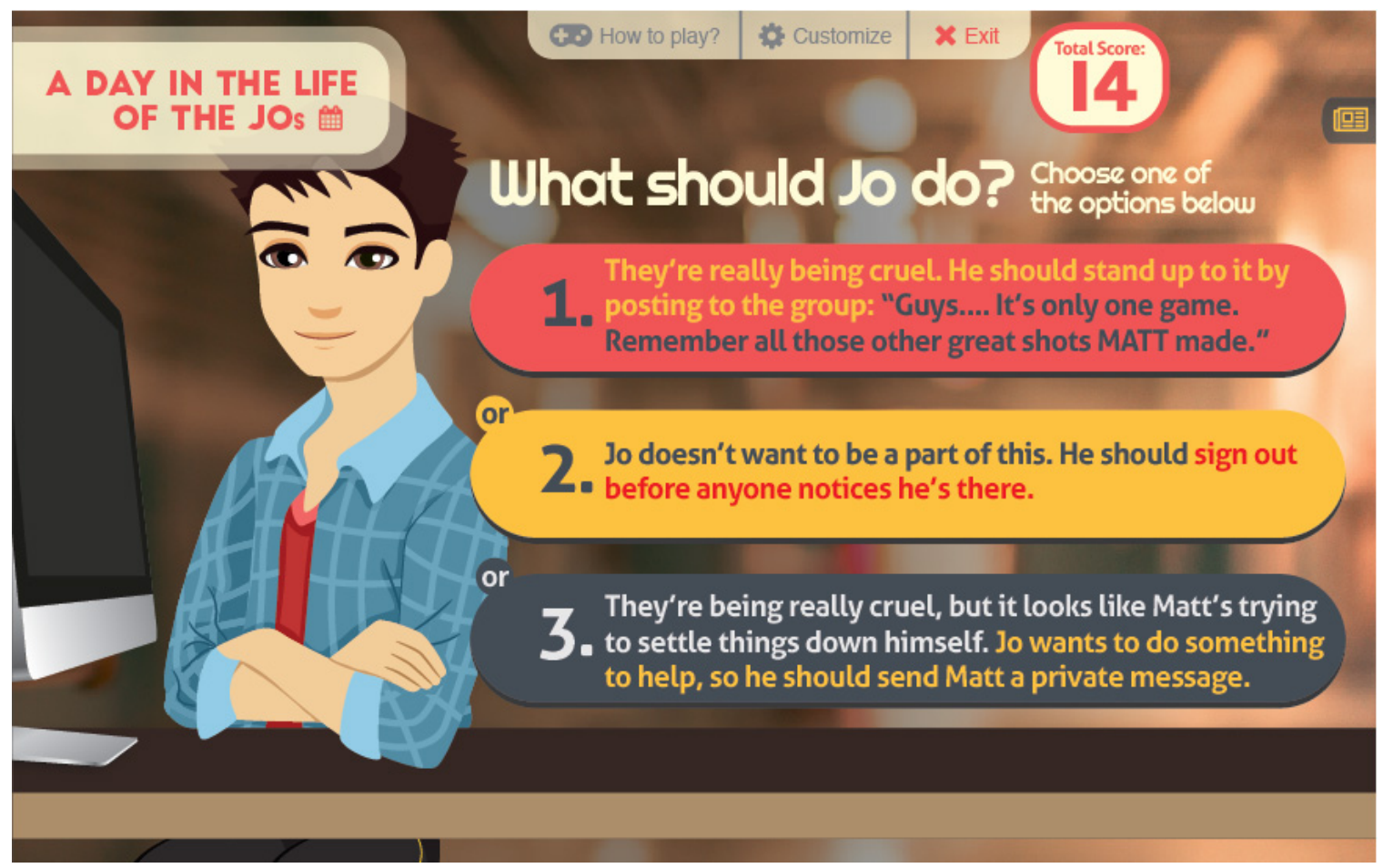

Figure A.19: D1-Cyberbullying: Scene 3 of fourth scenario.

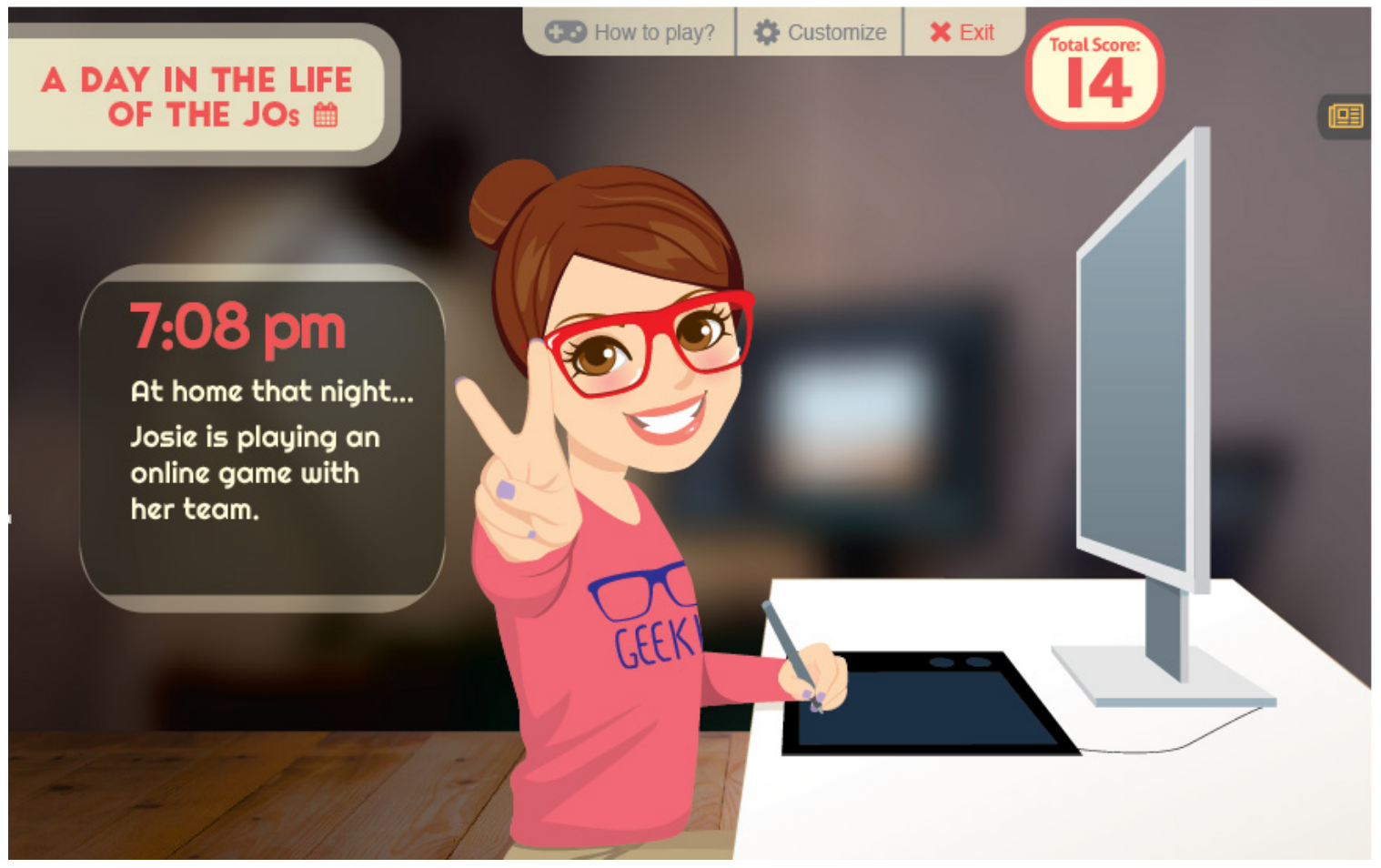

Figure A.20: D1-Cyberbullying: Scene 1 of fifth scenario. 


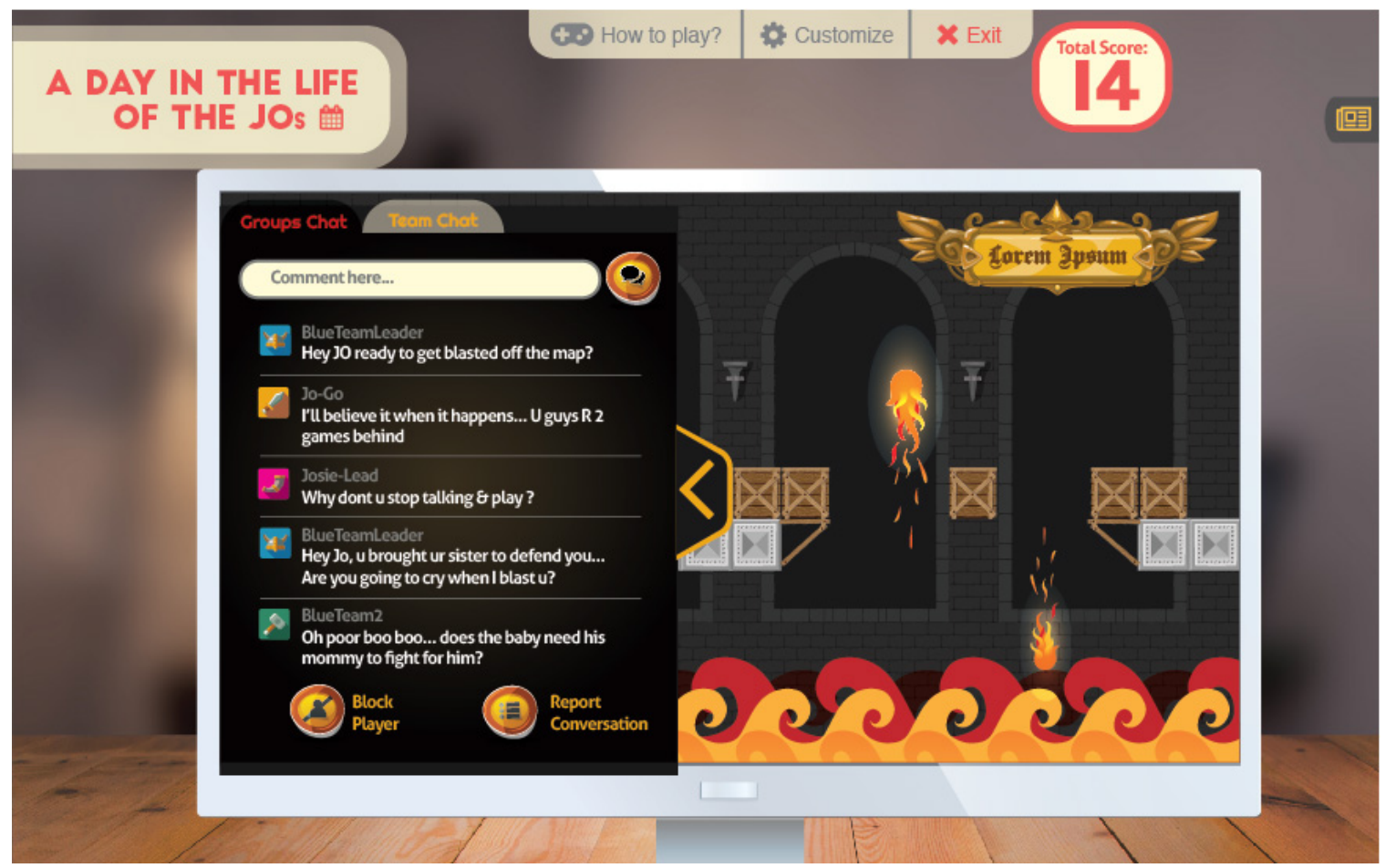

Figure A.21: D1-Cyberbullying: Scene 2 of fifth scenario.

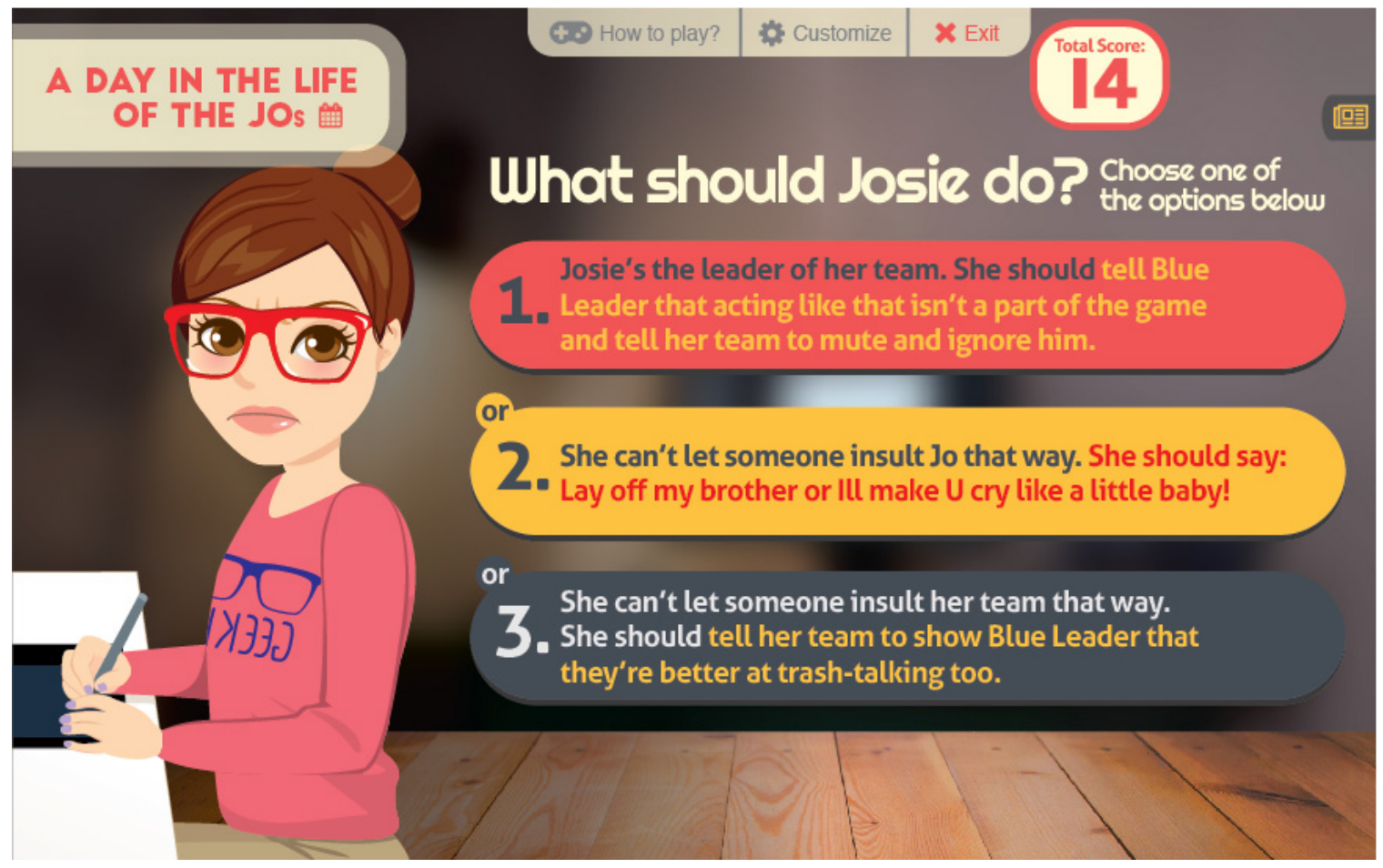

Figure A.22: D1-Cyberbullying: Scene 3 of fifth scenario. 


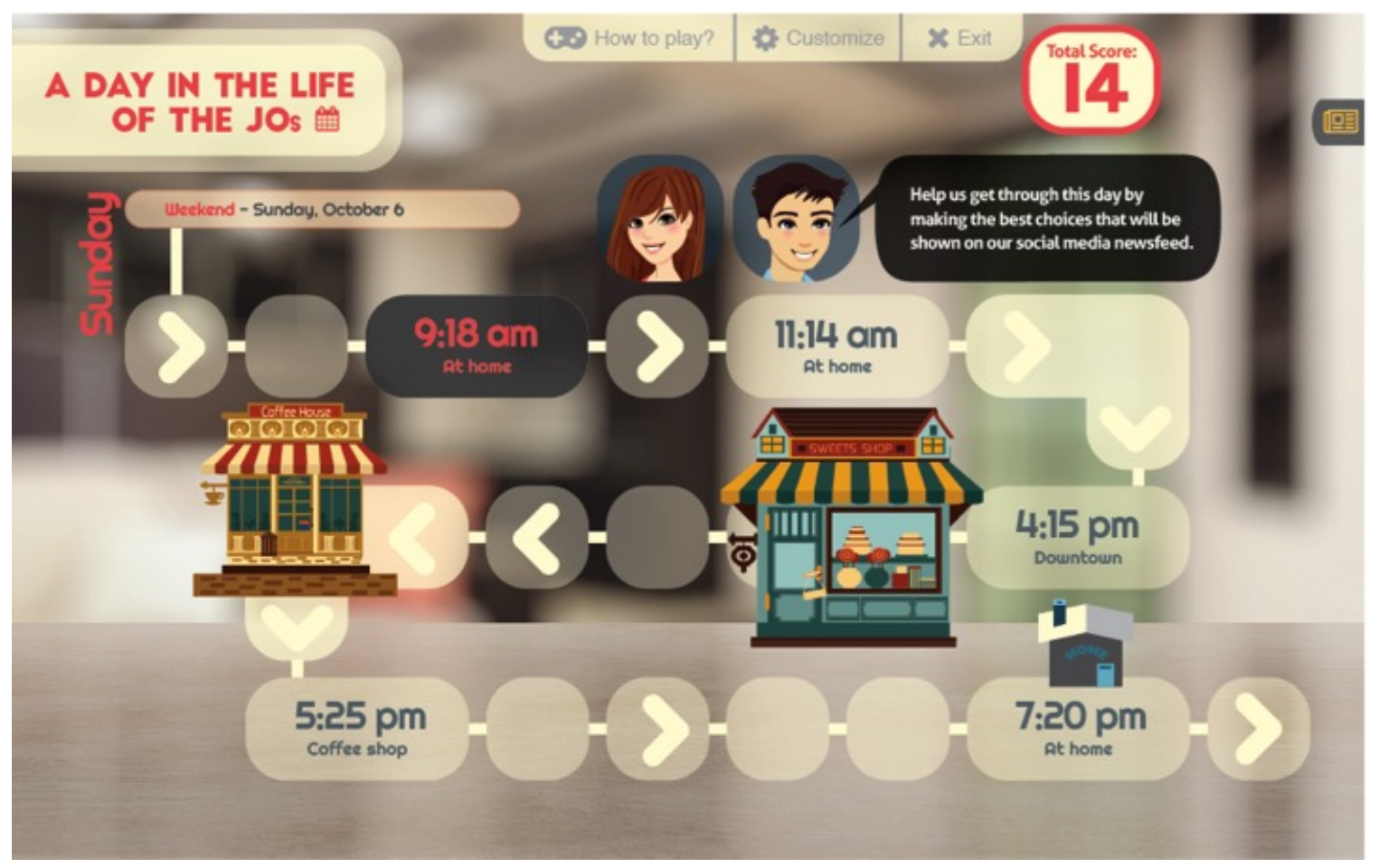

Figure A.23: The Day View of D2-Data mining topic.

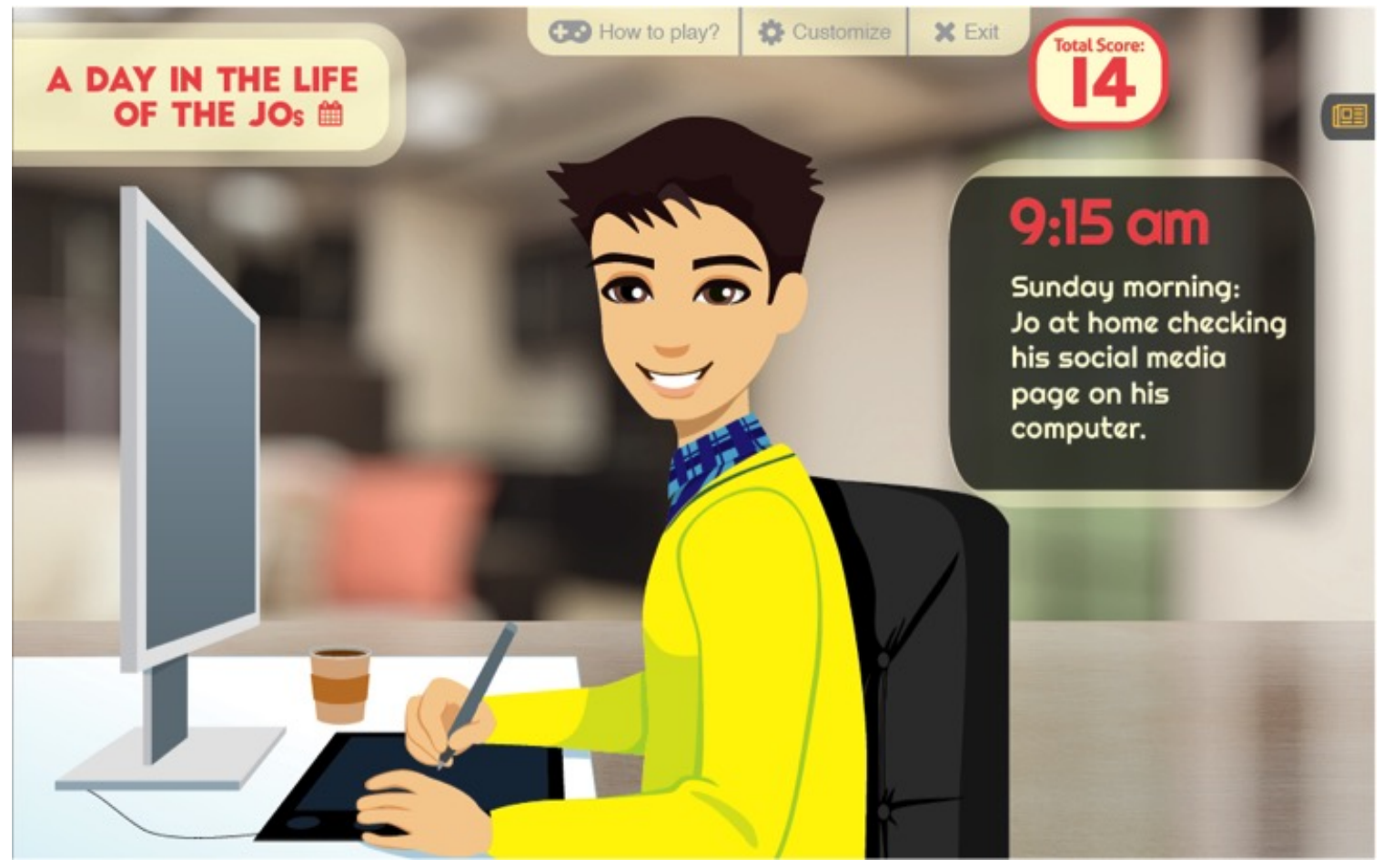

Figure A.24: D2-Data mining: Scene 1 of first scenario. 


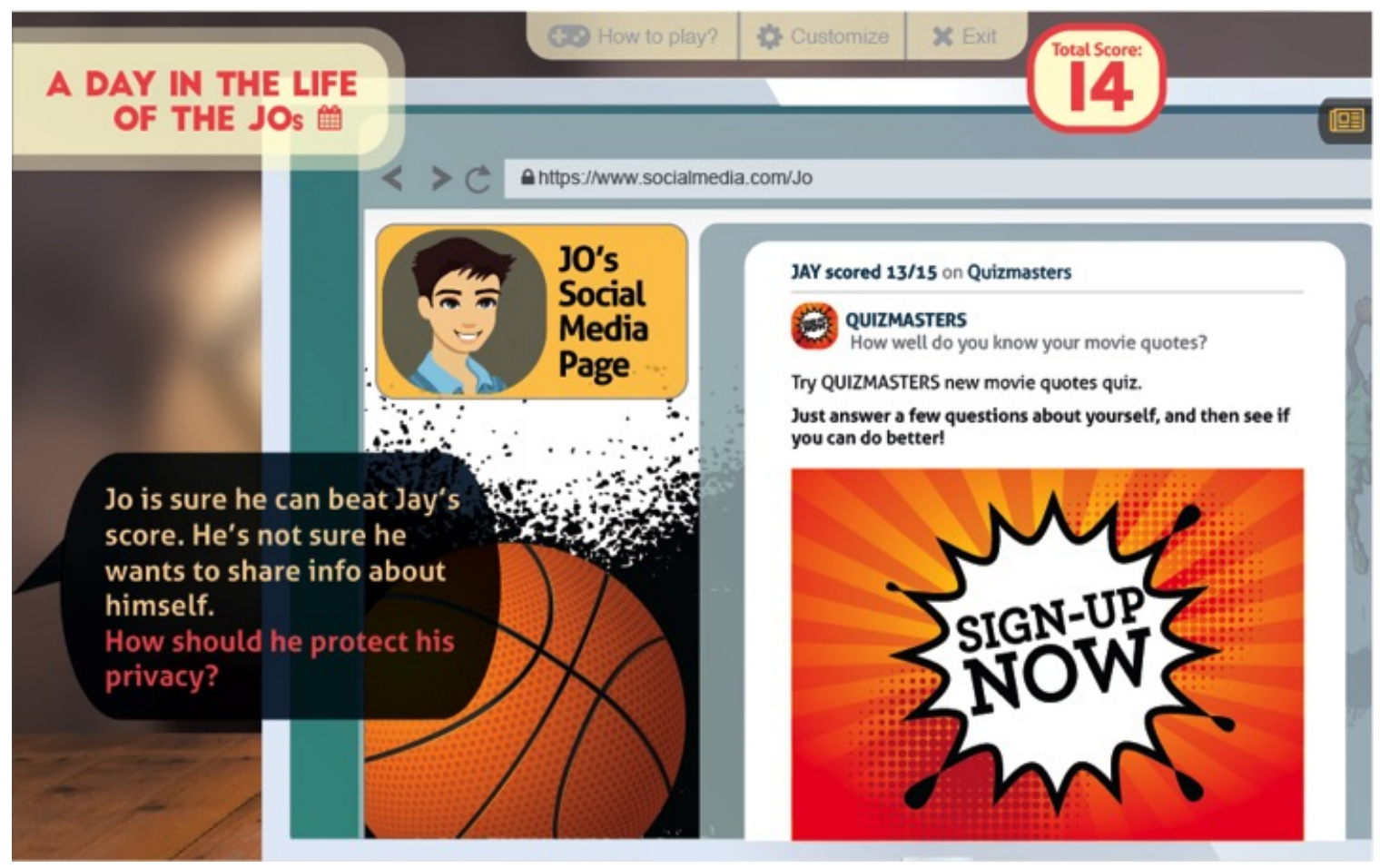

Figure A.25: D2-Data mining: Scene 2 of first scenario.

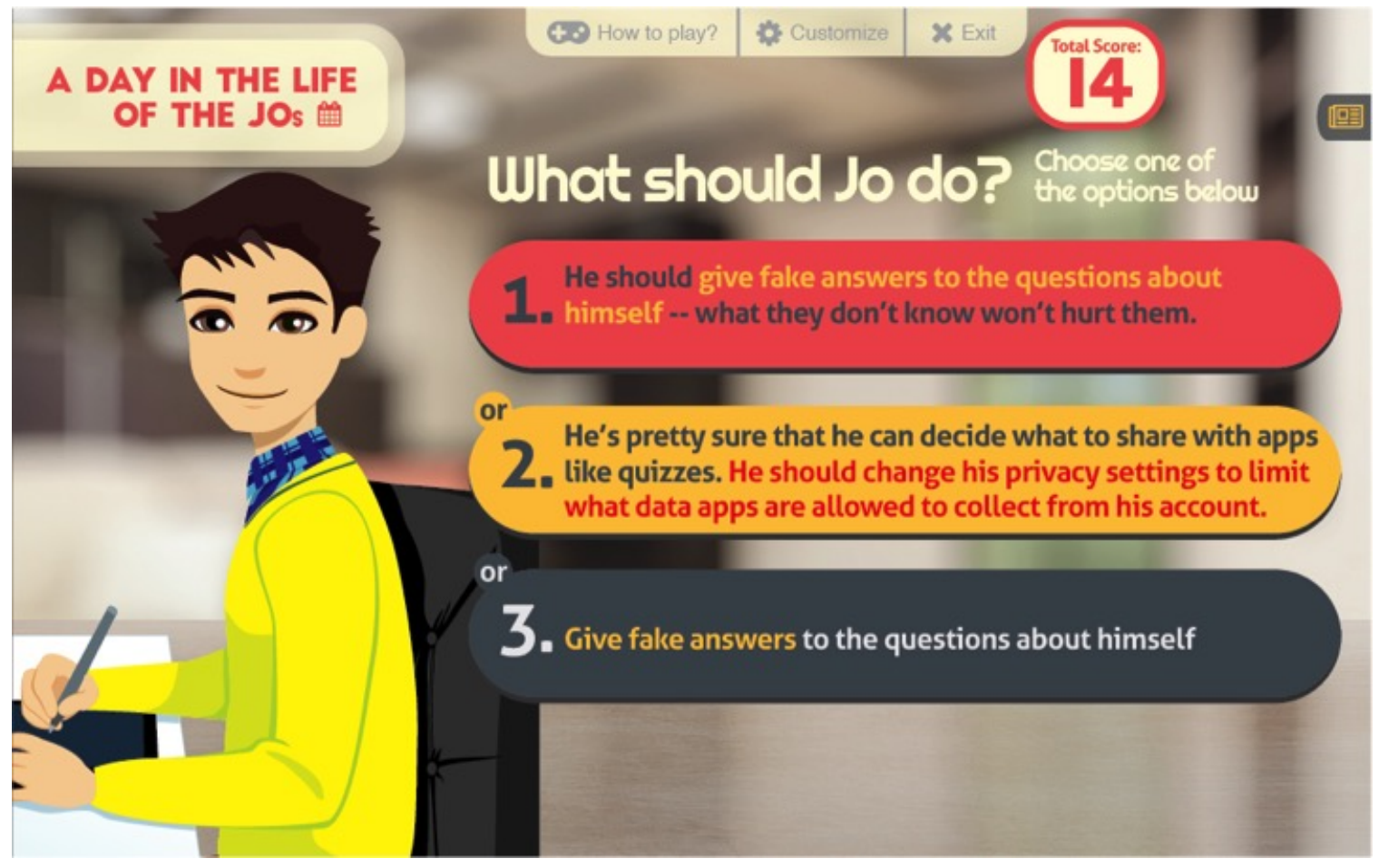

Figure A.26: D2-Data mining: Scene 3 of first scenario. 


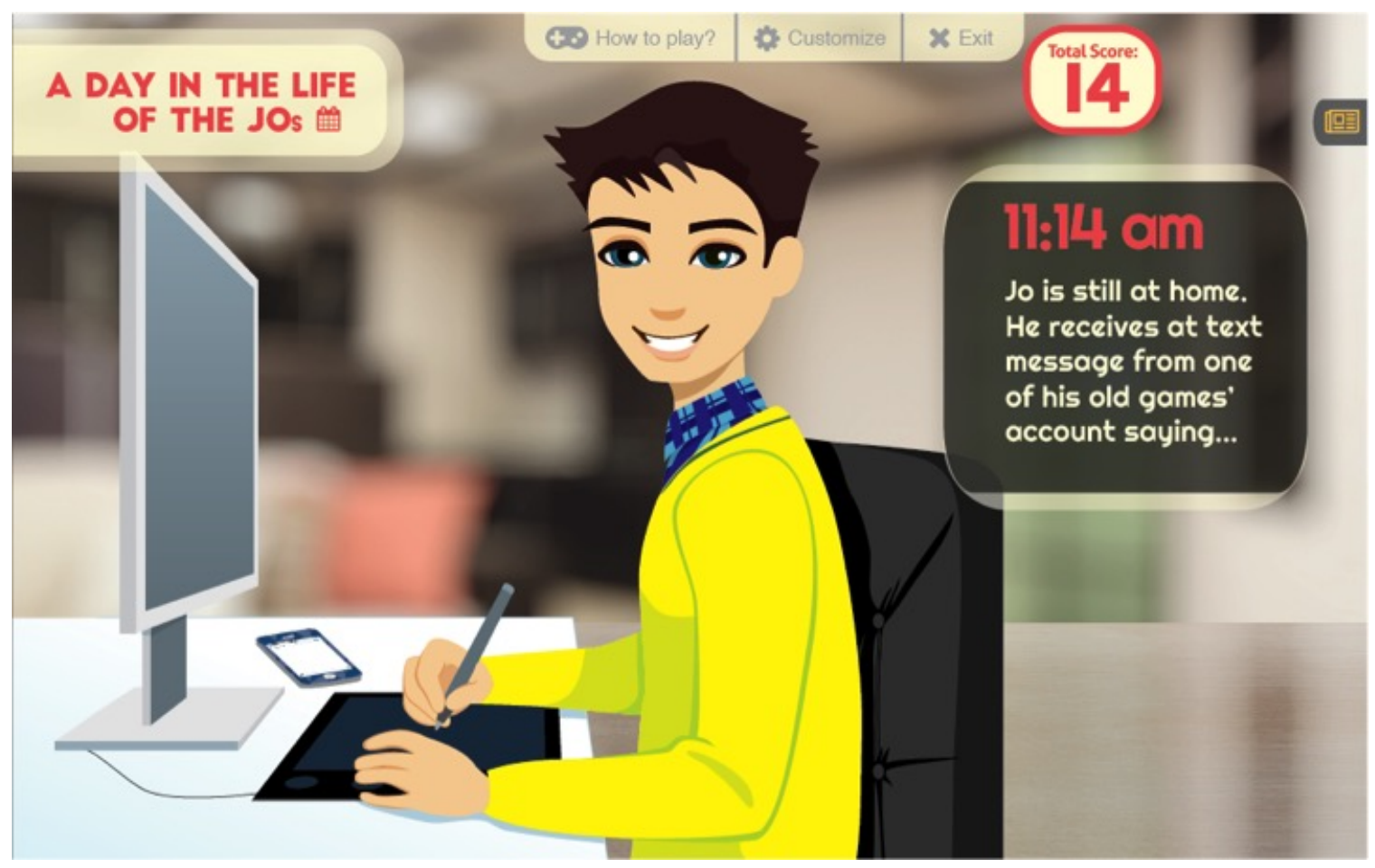

Figure A.27: D2-Data mining: Scene 1 of second scenario.

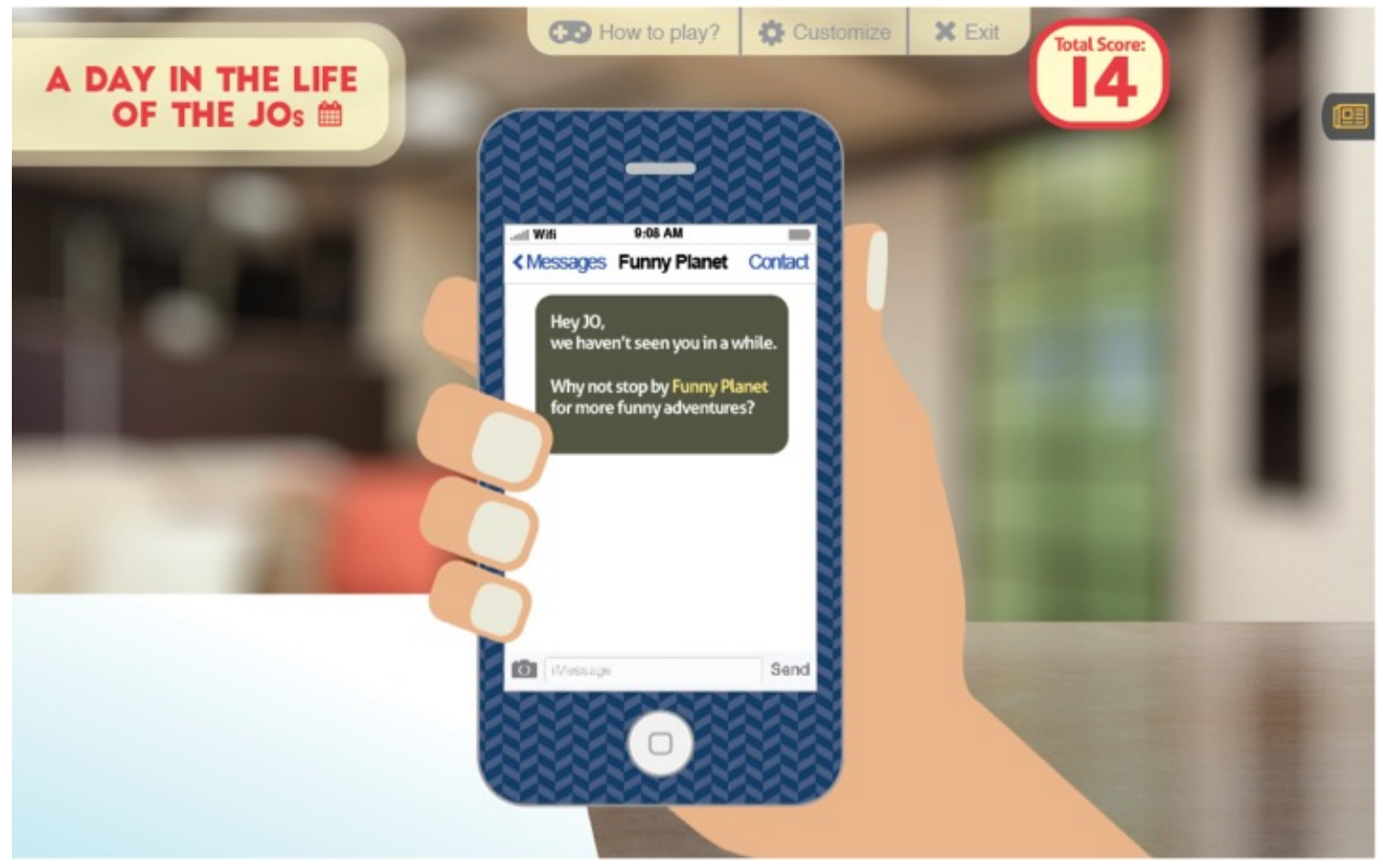

Figure A.28: D2-Data mining: Scene 2 of second scenario. 


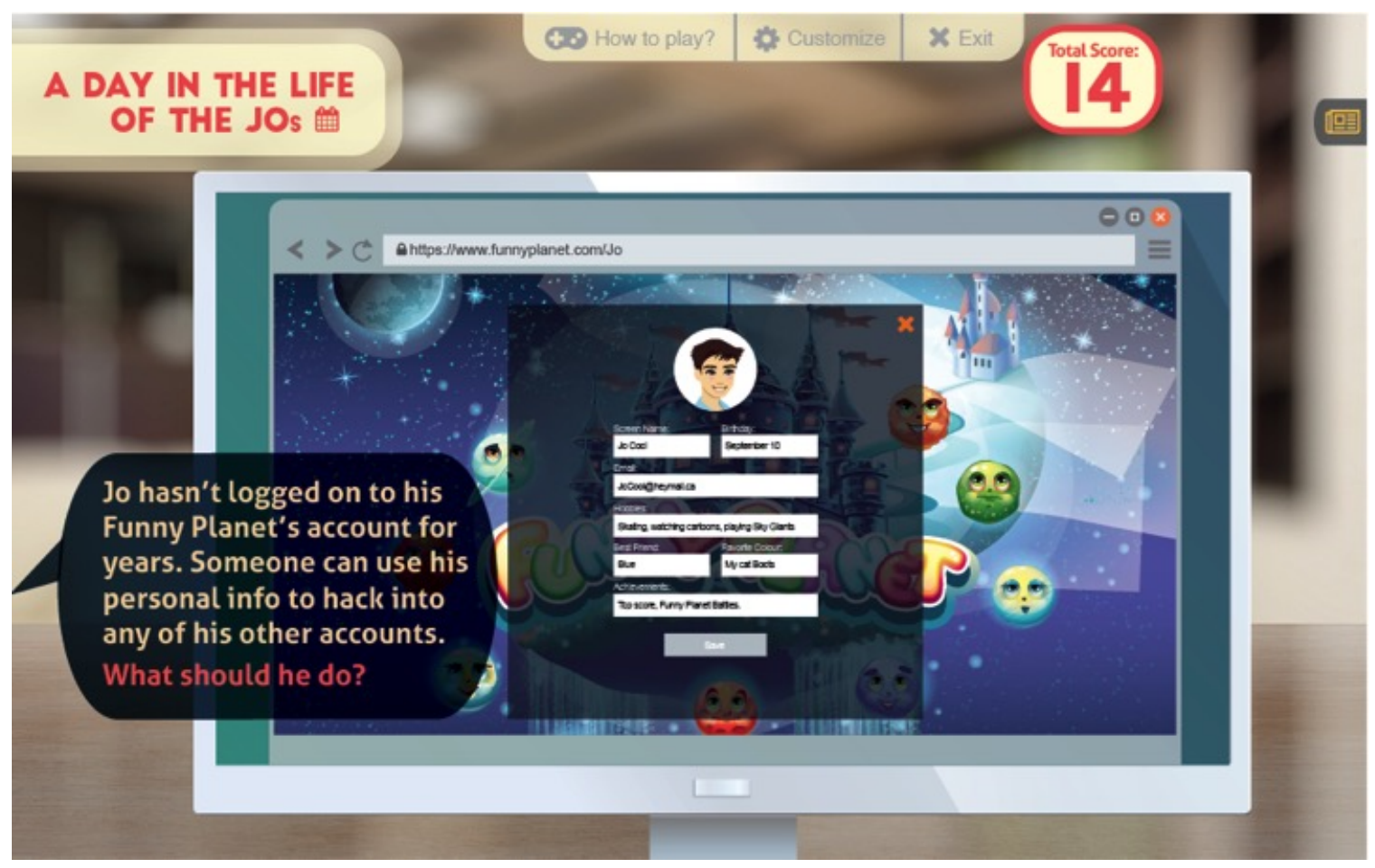

Figure A.29: D2-Data mining: Scene 3 of second scenario.

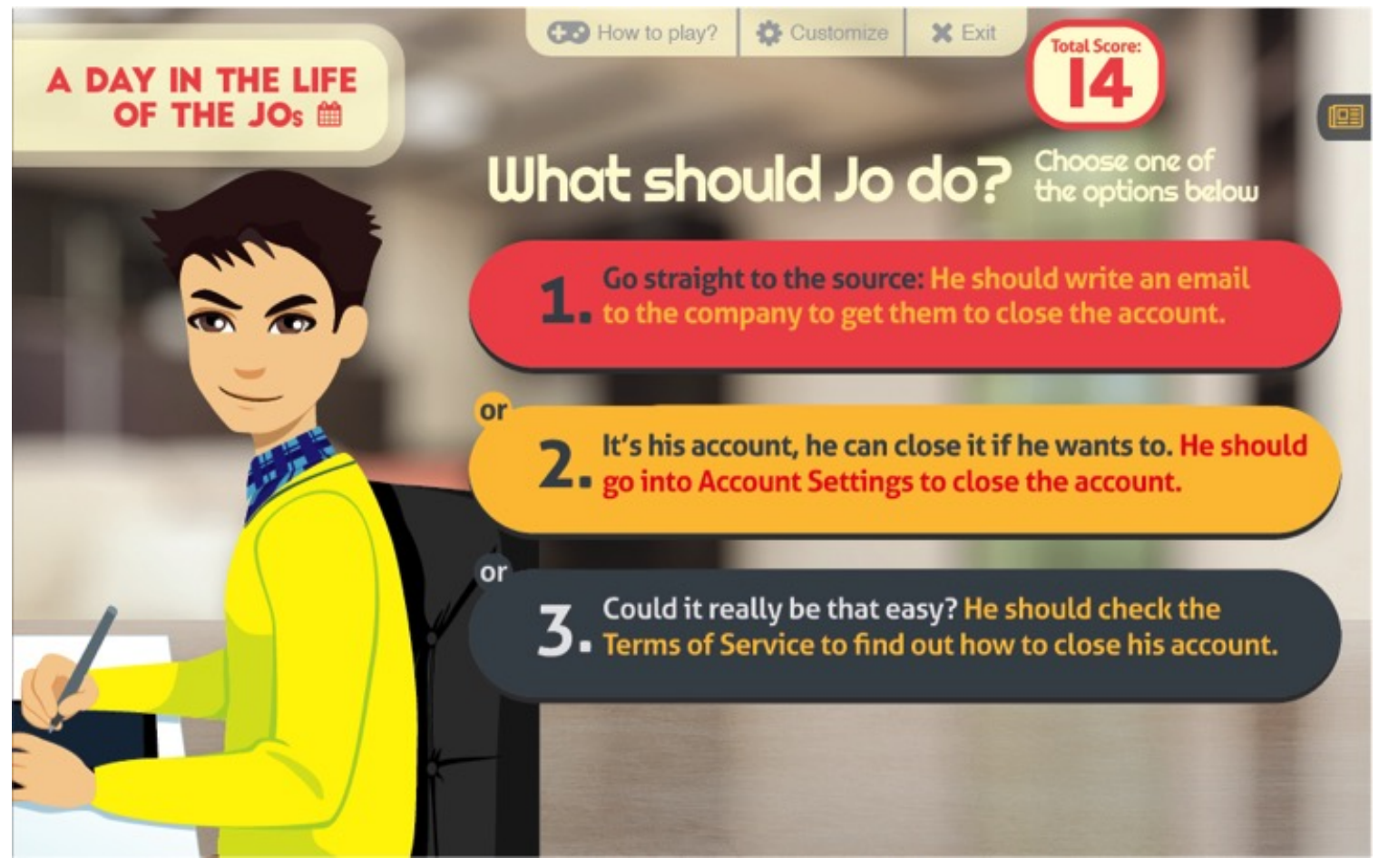

Figure A.30: D2-Data mining: Scene 4 of second scenario. 


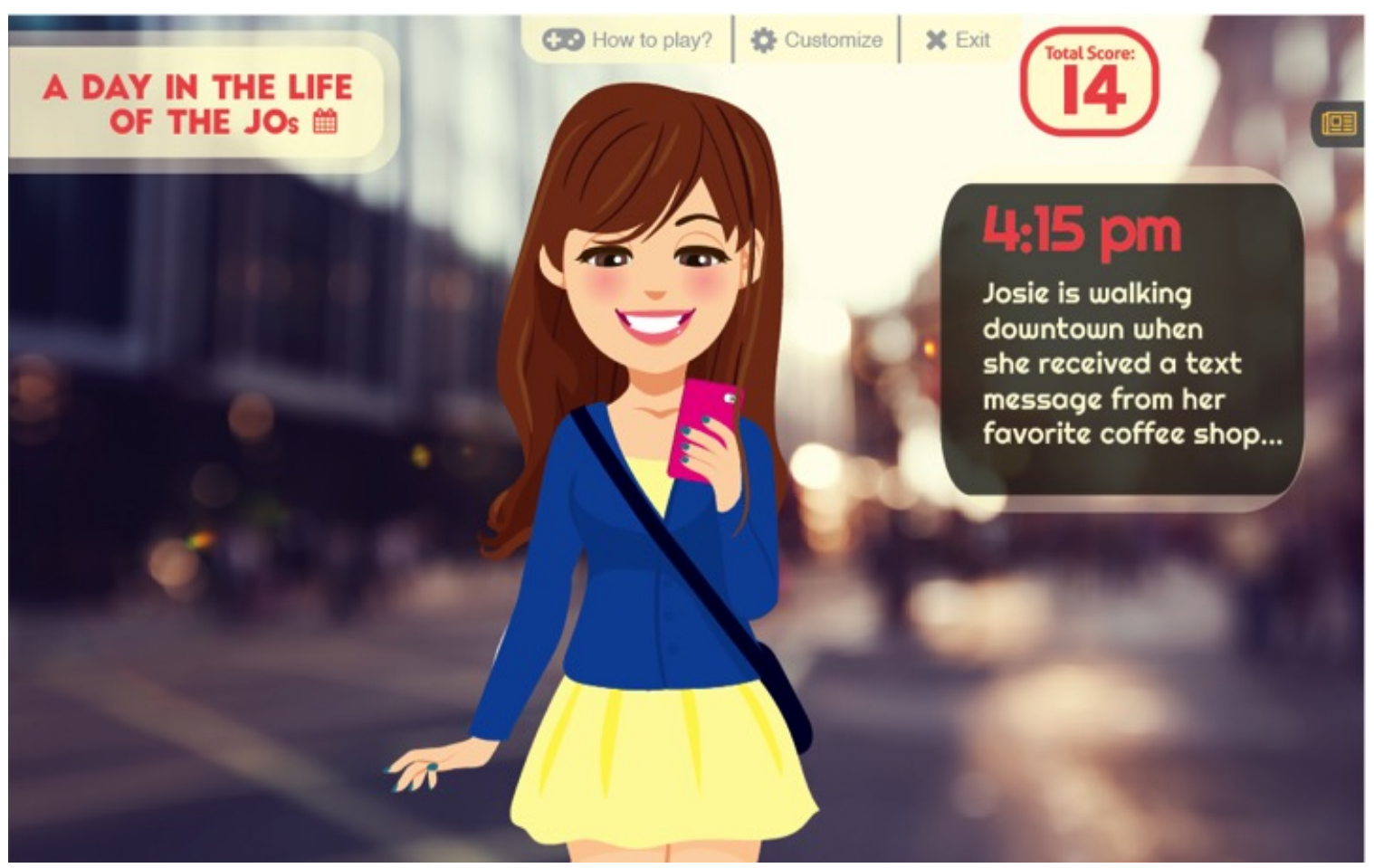

Figure A.31: D2-Data mining: Scene 1 of third scenario.

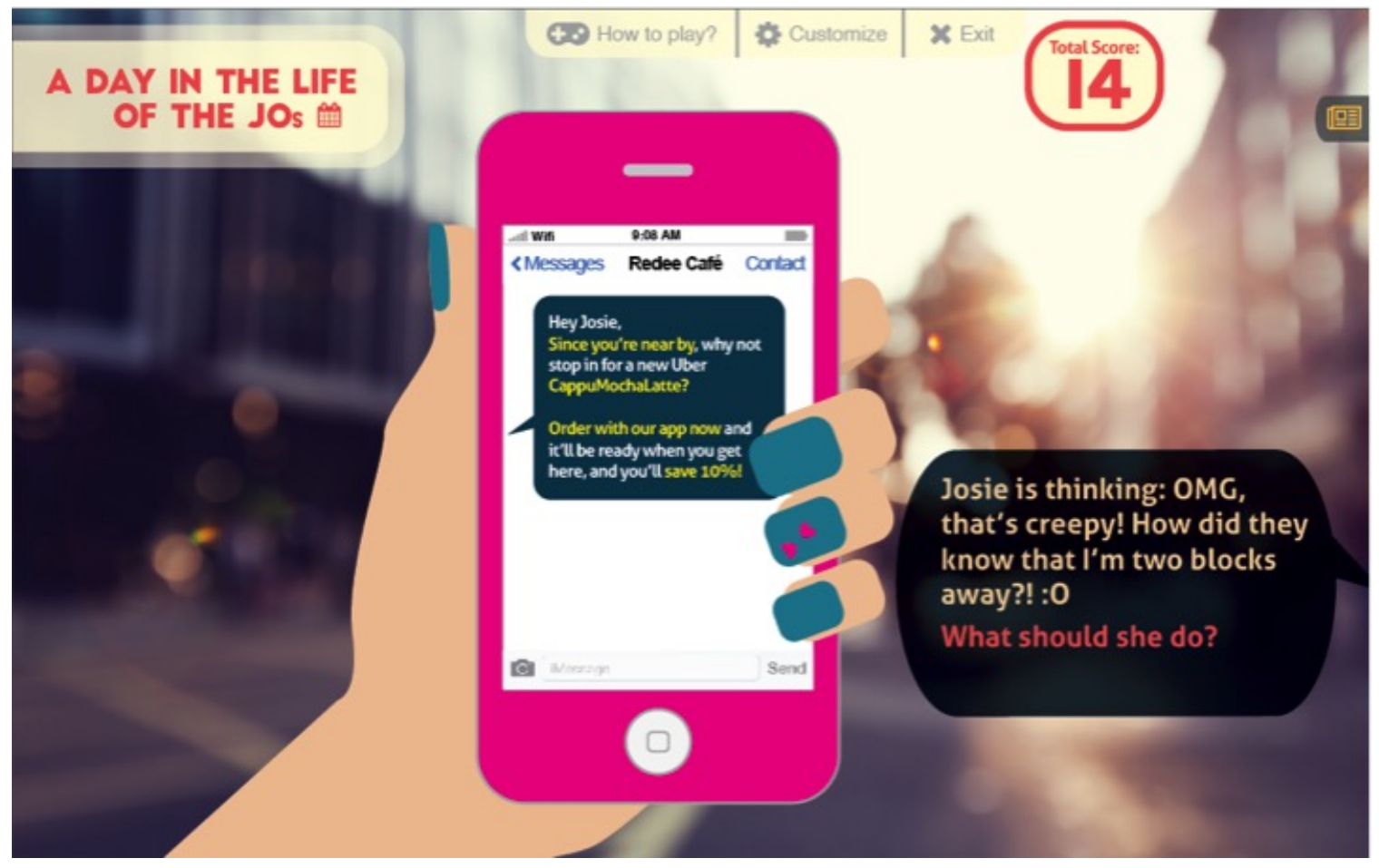

Figure A.32: D2-Data mining: Scene 2 of third scenario. 


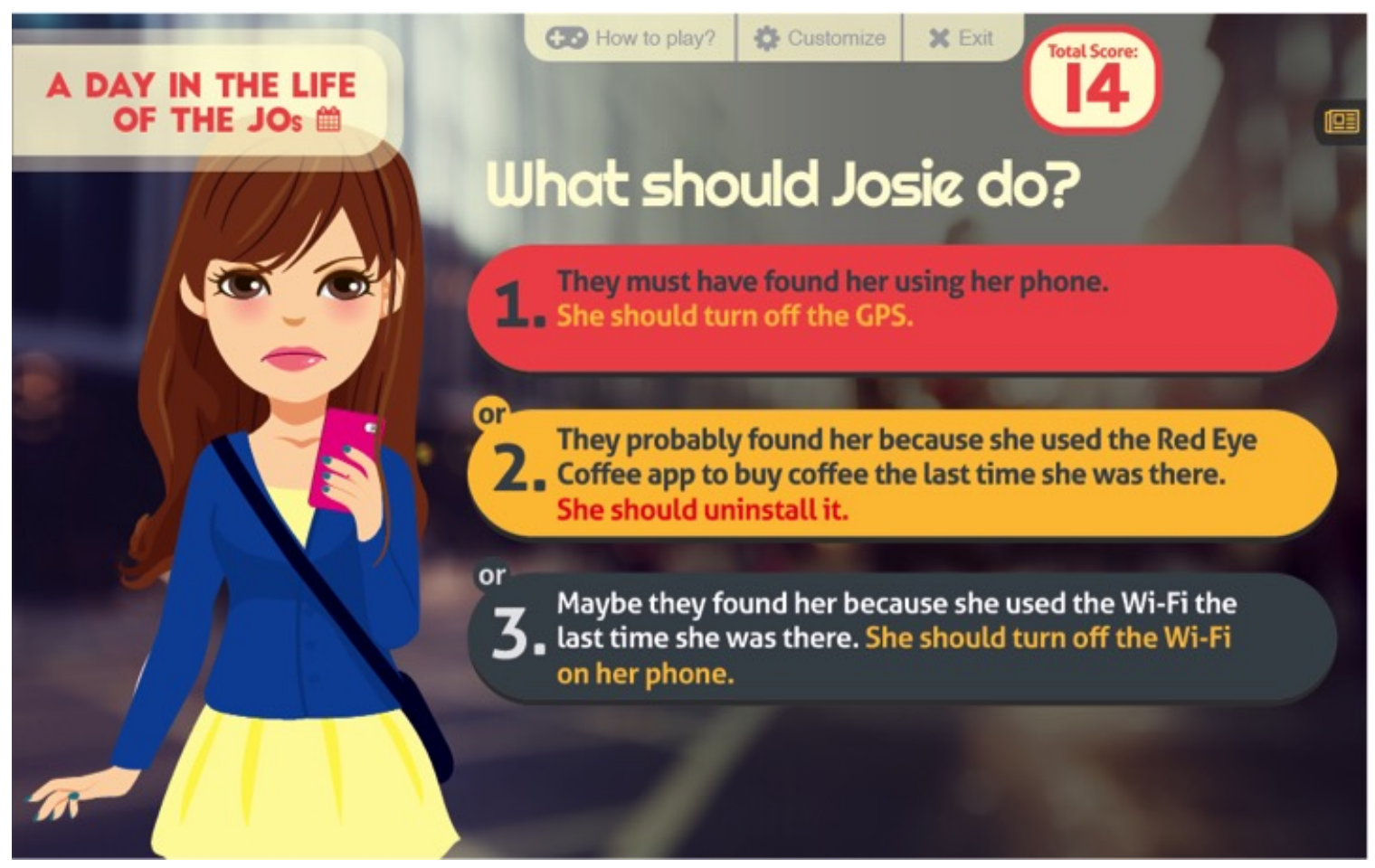

Figure A.33: D2-Data mining: Scene 3 of third scenario.

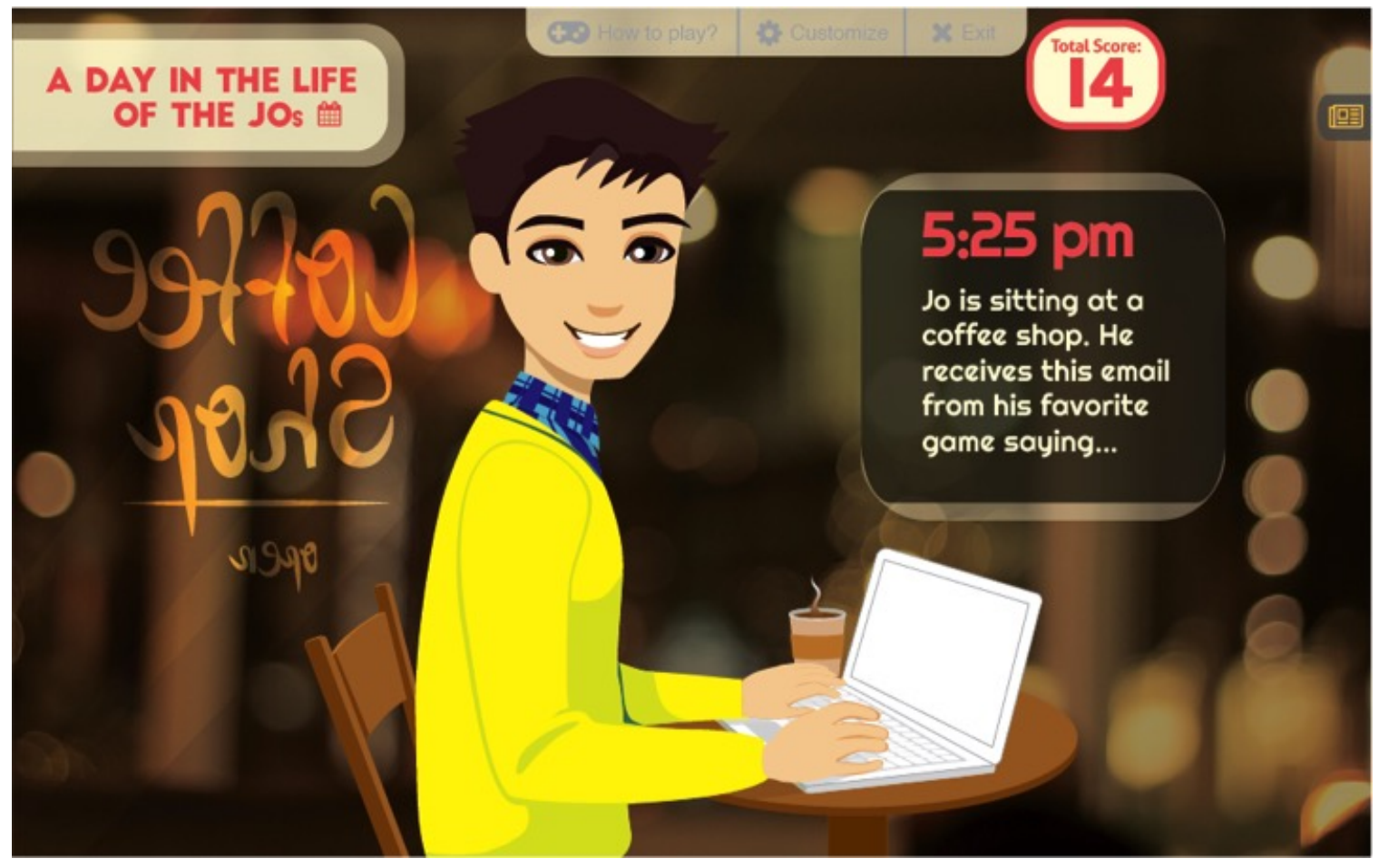

Figure A.34: D2-Data mining: Scene 1 of fourth scenario. 


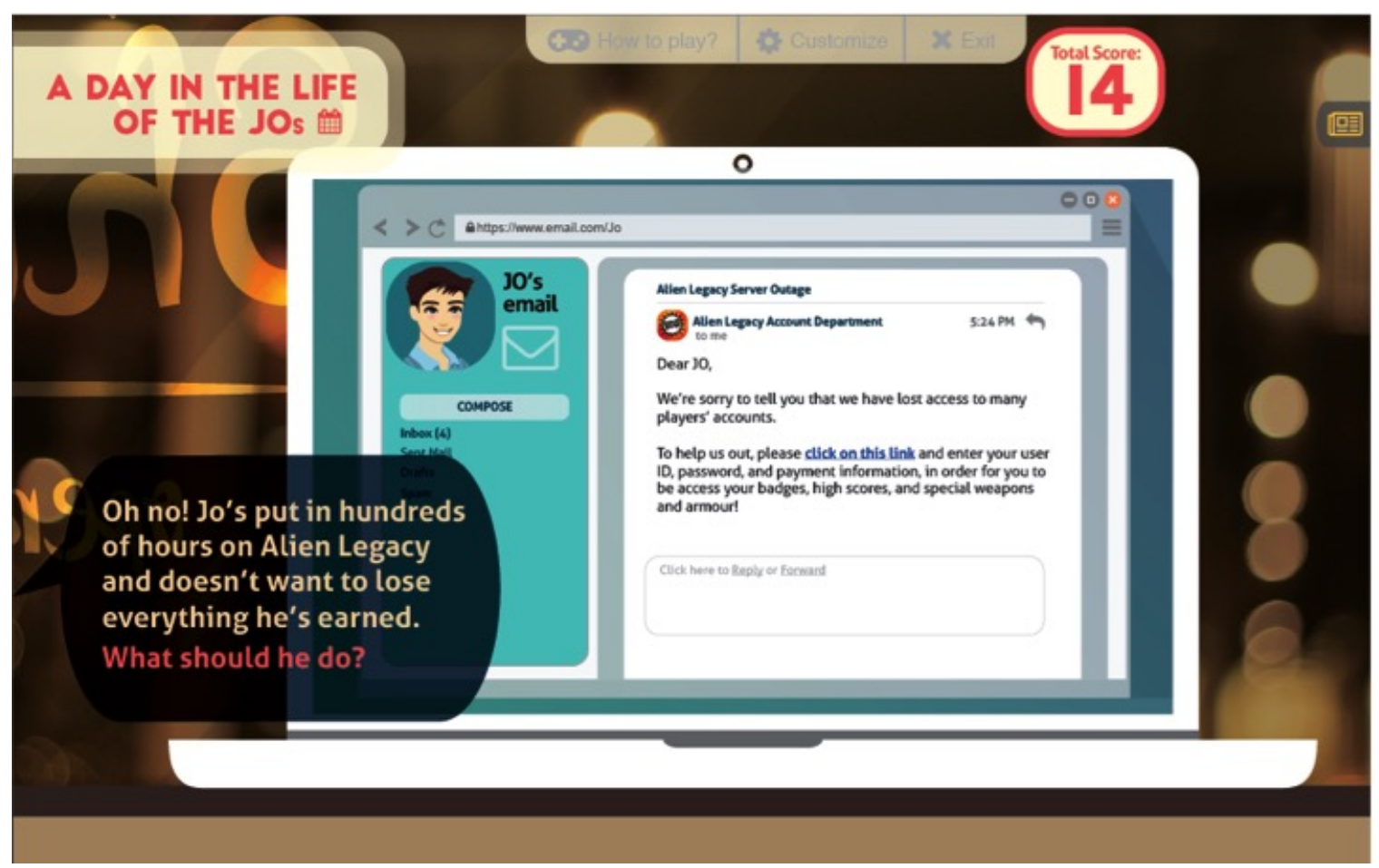

Figure A.35: D2-Data mining: Scene 2 of fourth scenario.

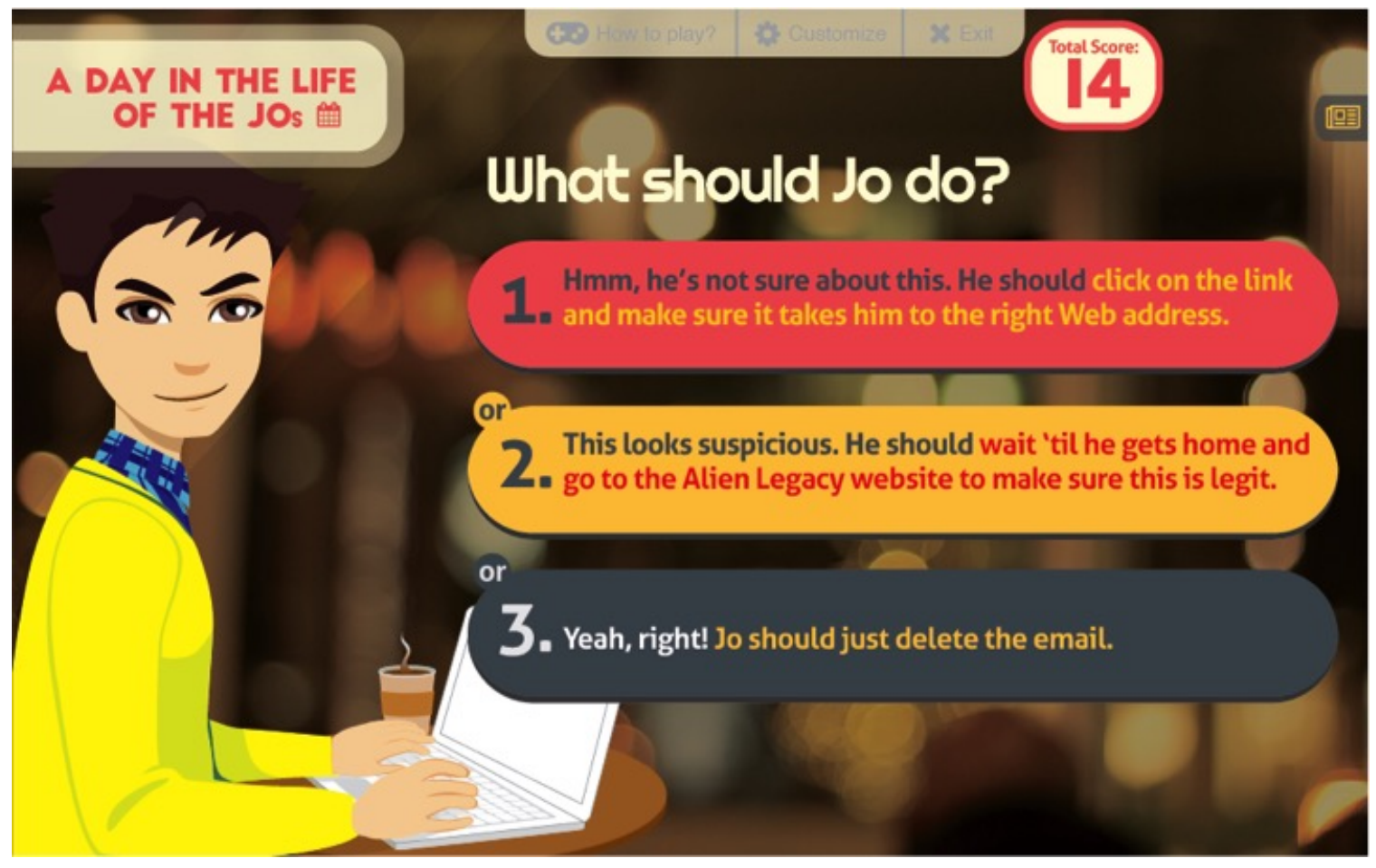

Figure A.36: D2-Data mining: Scene 3 of fourth scenario. 


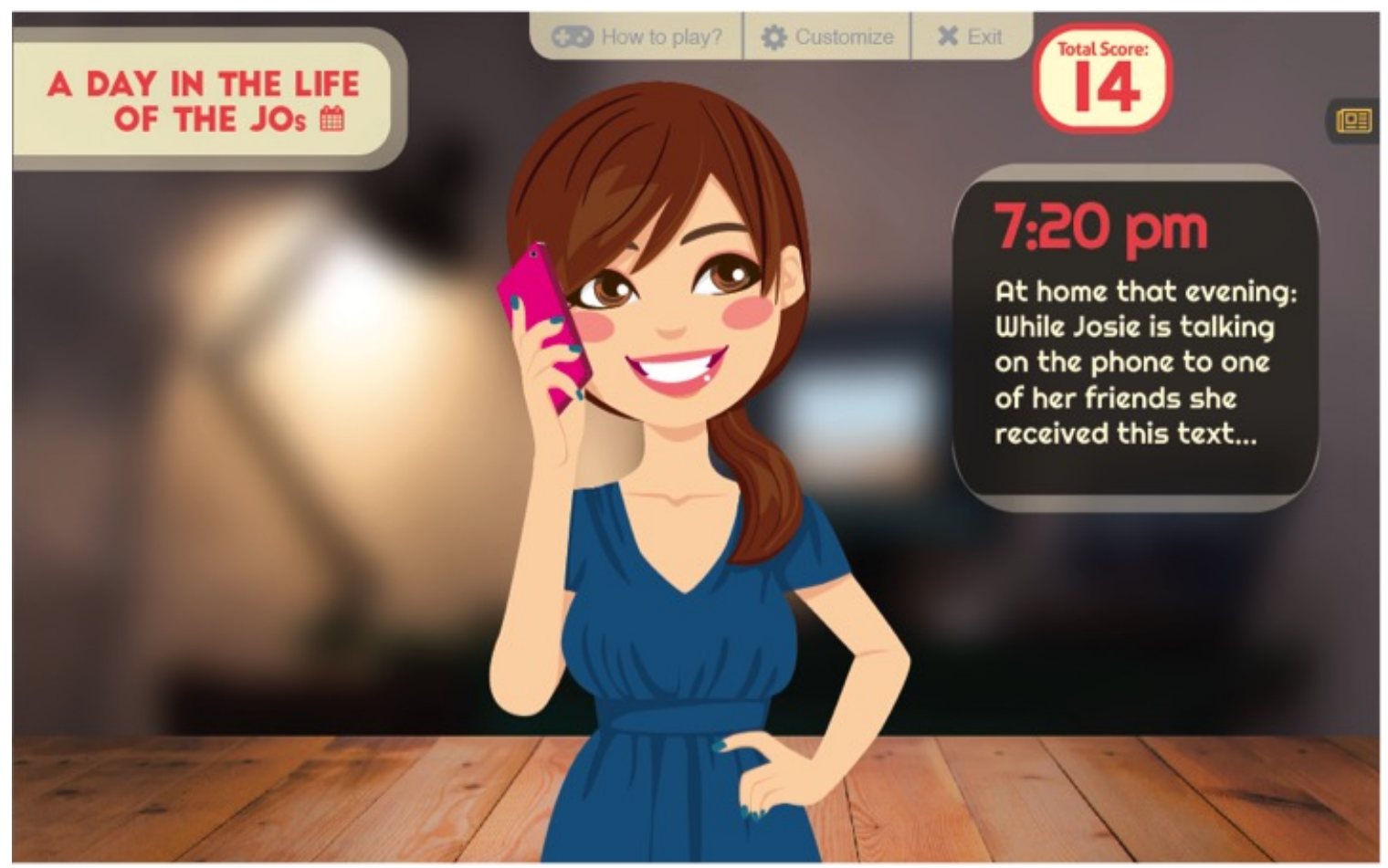

Figure A.37: D2-Data mining: Scene 1 of fifth scenario.

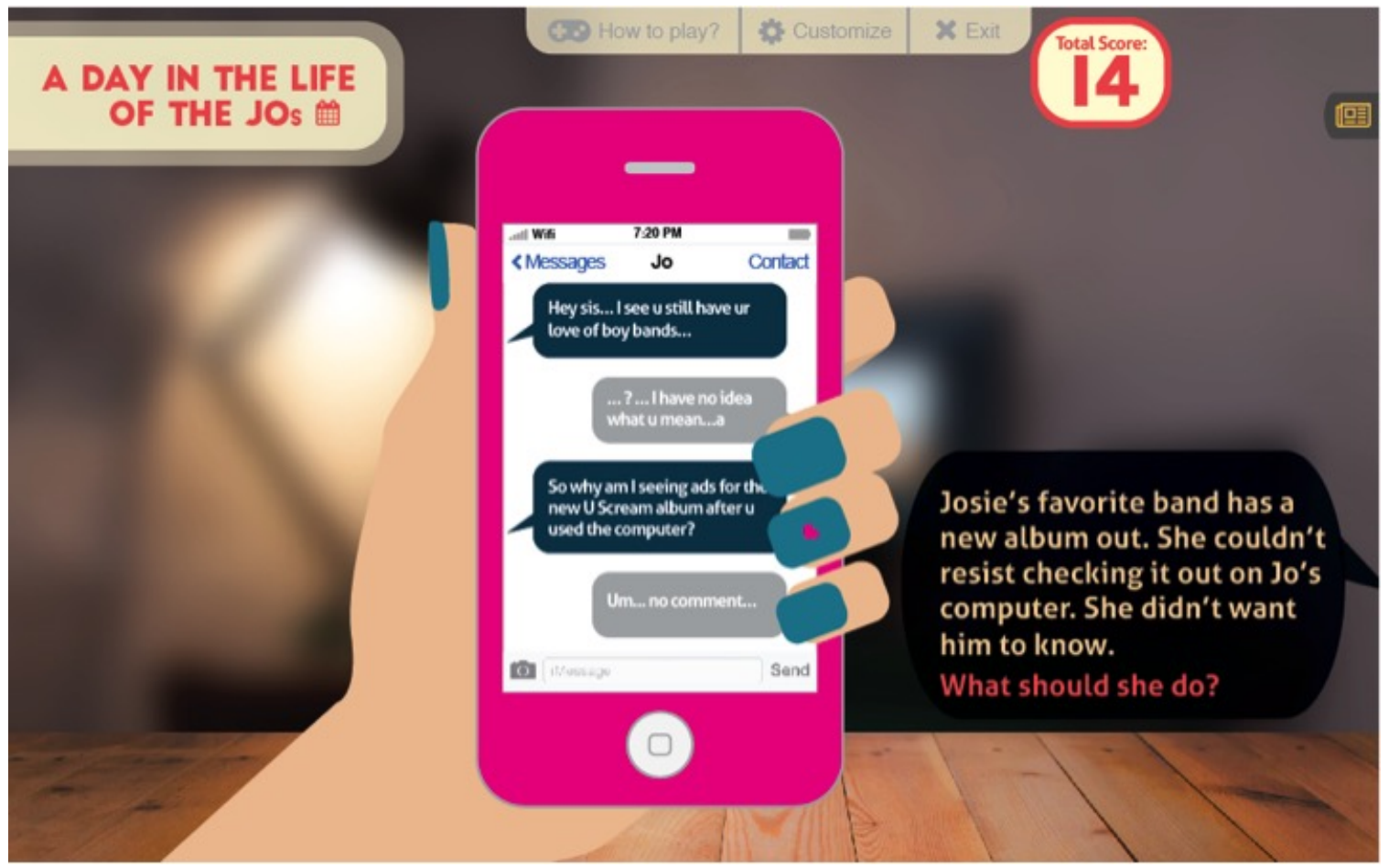

Figure A.38: D2-Data mining: Scene 2 of fifth scenario. 


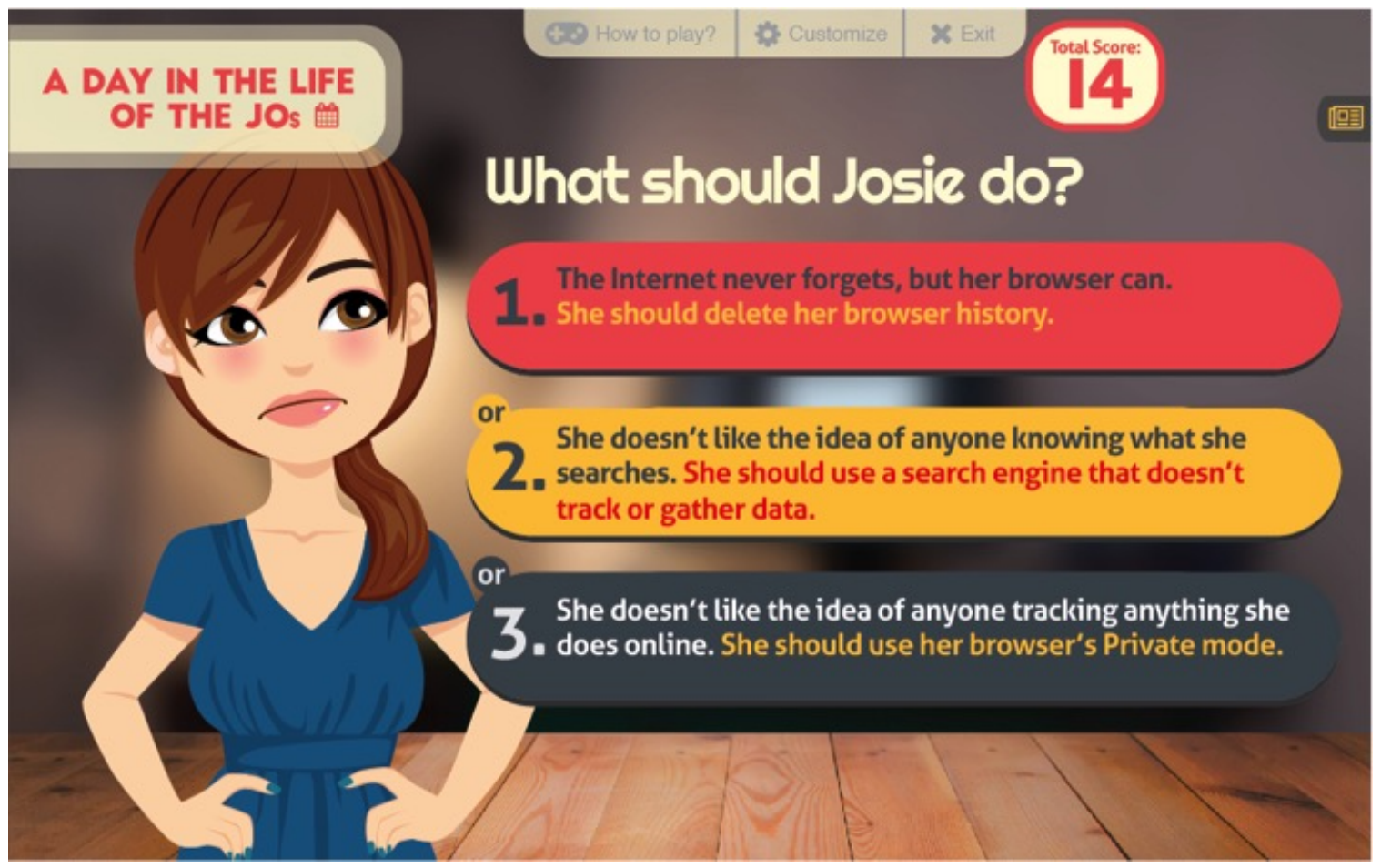

Figure A.39: D2-Data mining: Scene 3 of fifth scenario. 


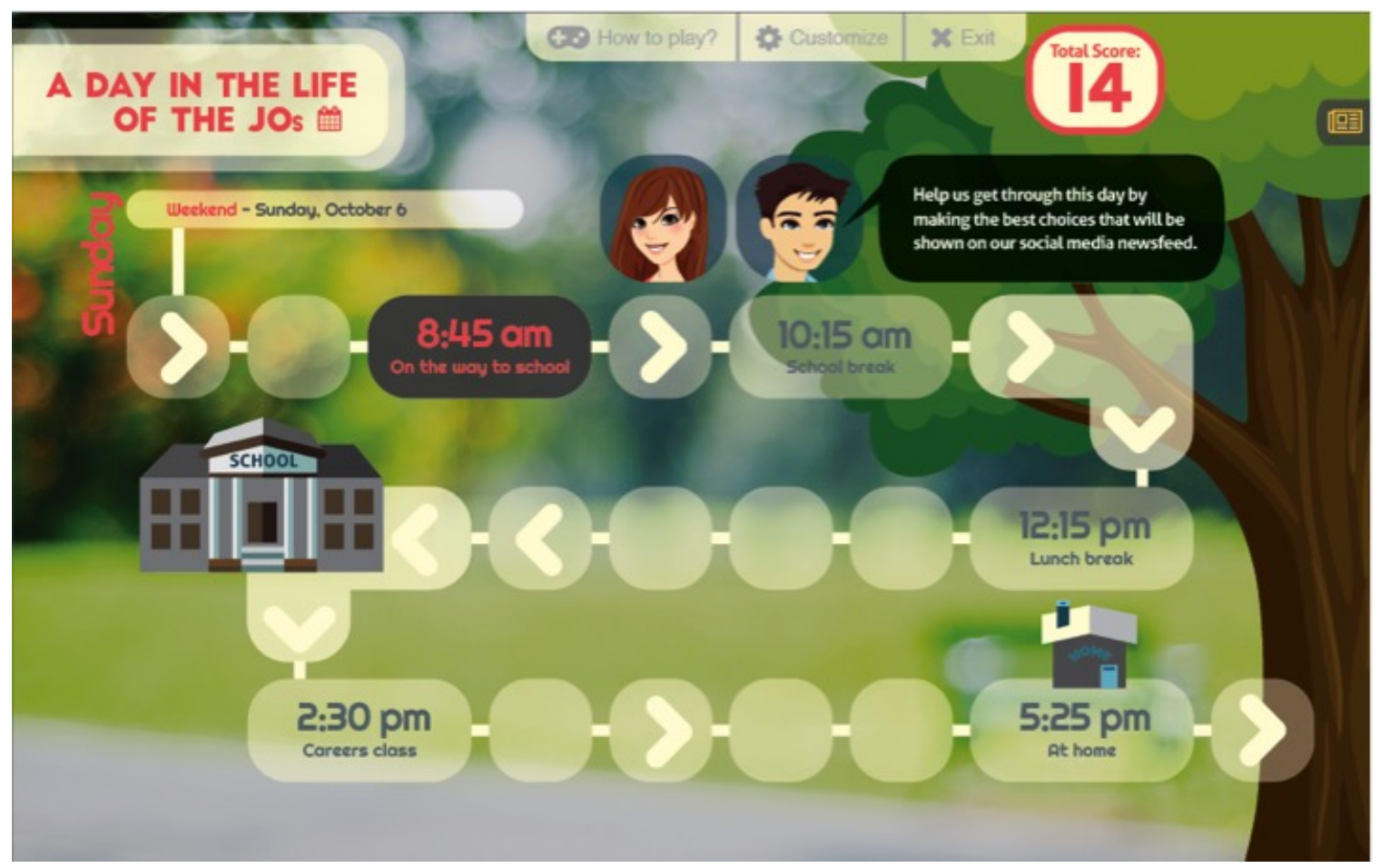

Figure A.40: The Day View of D3-Internet privacy topic.

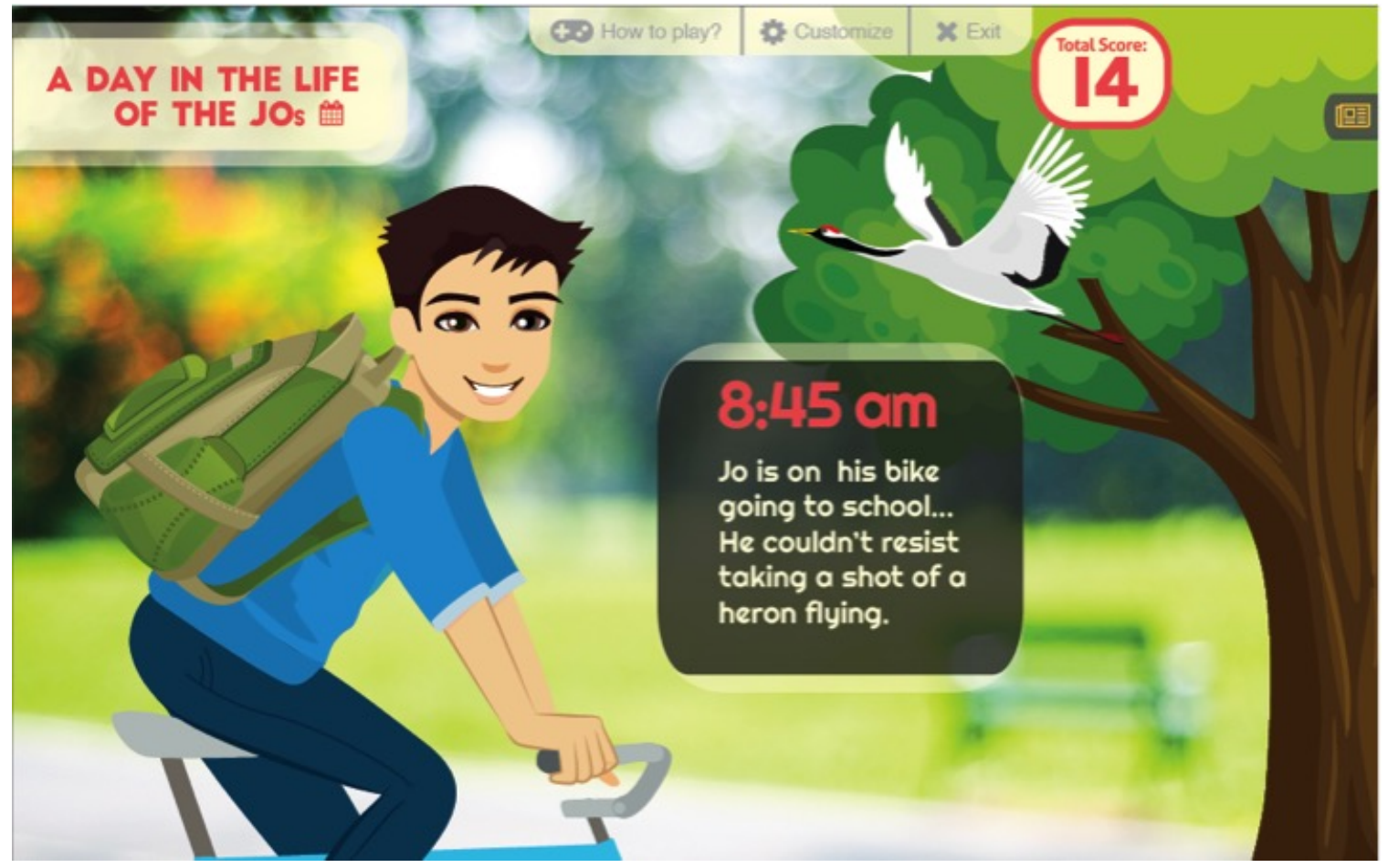

Figure A.41: D3-Internet privacy: Scene 1 of first scenario. 


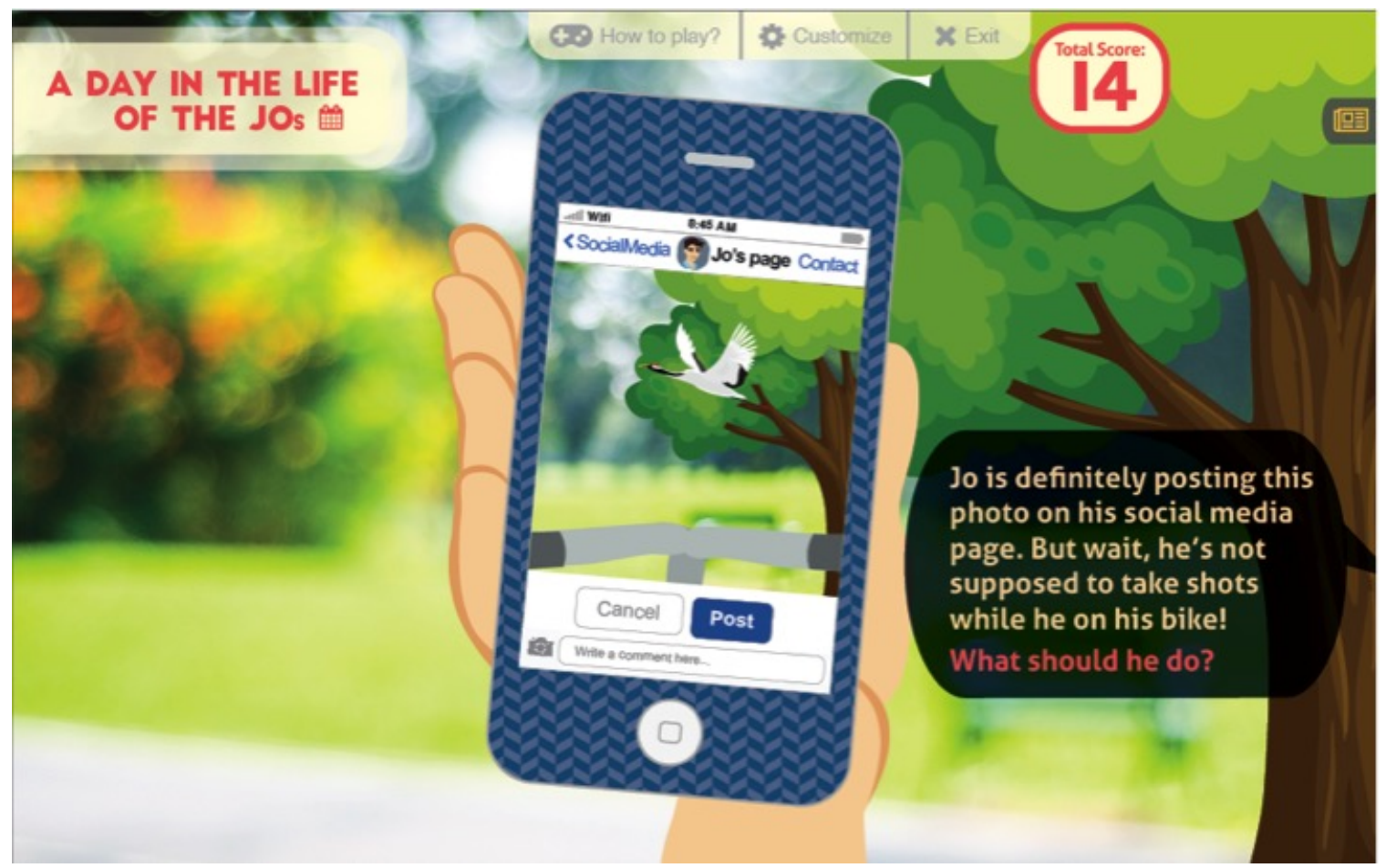

Figure A.42: D3-Internet privacy: Scene 2 of first scenario.

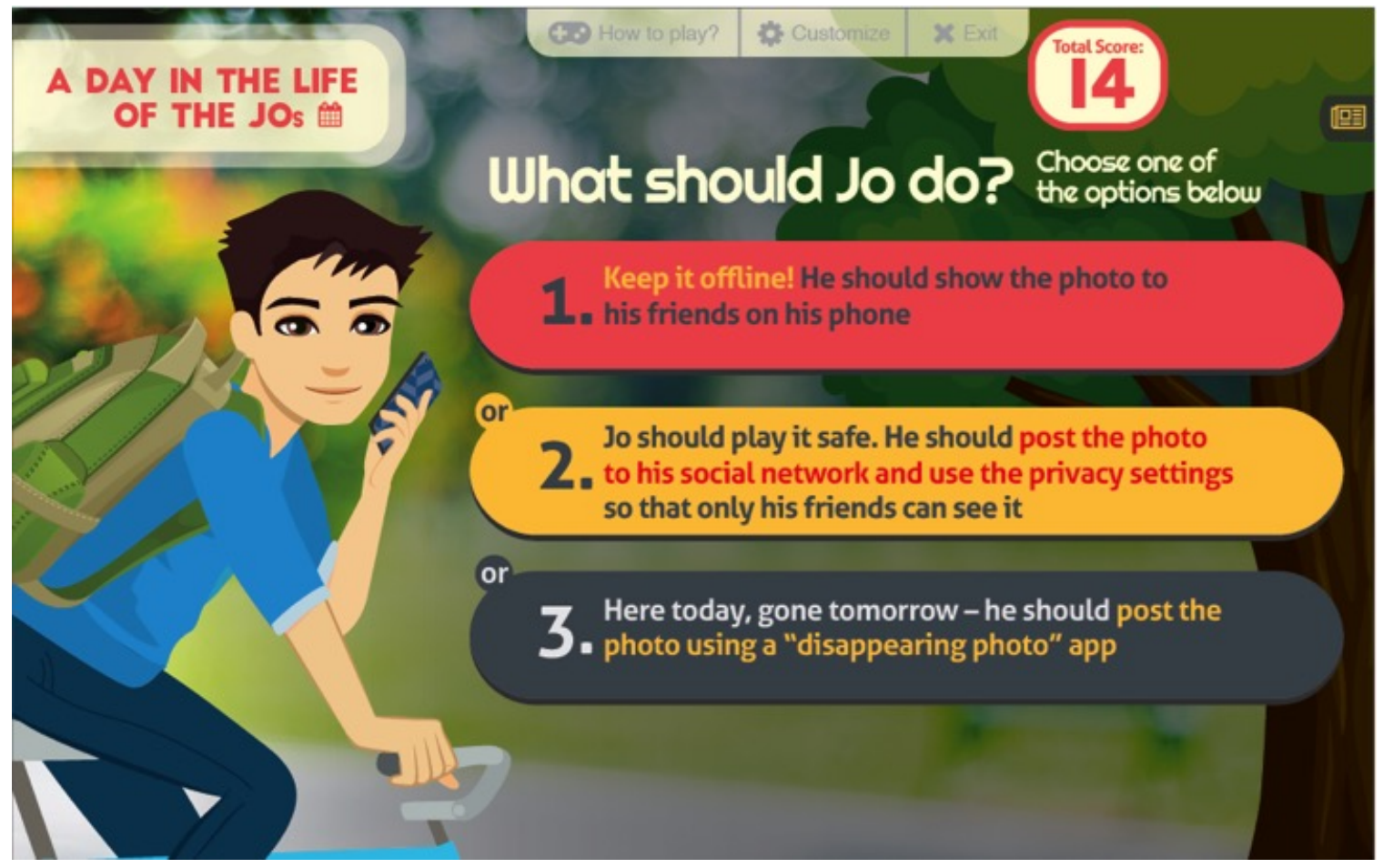

Figure A.43: D3-Internet privacy: Scene 3 of first scenario. 


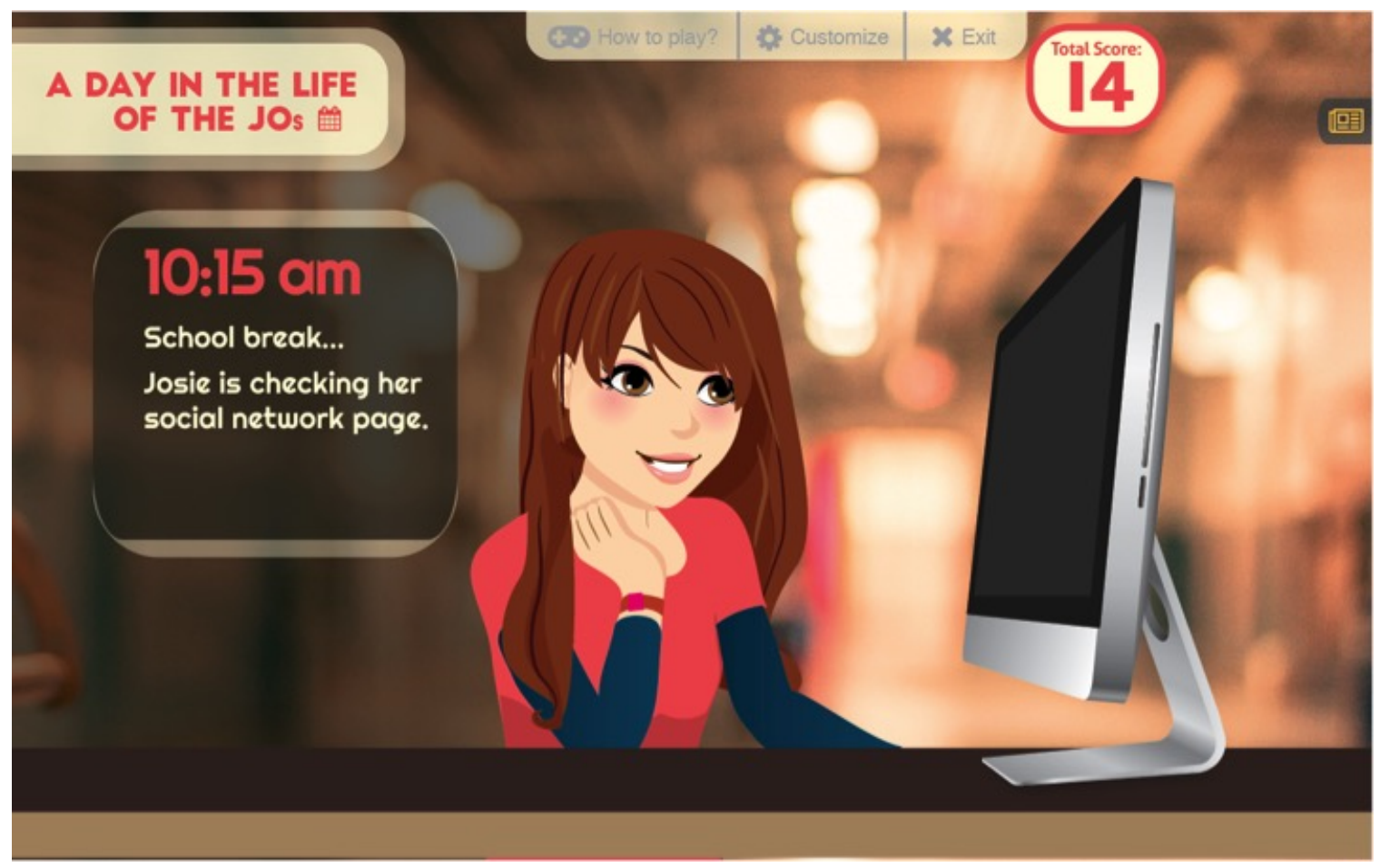

Figure A.44: D3-Internet privacy: Scene 1 of second scenario.

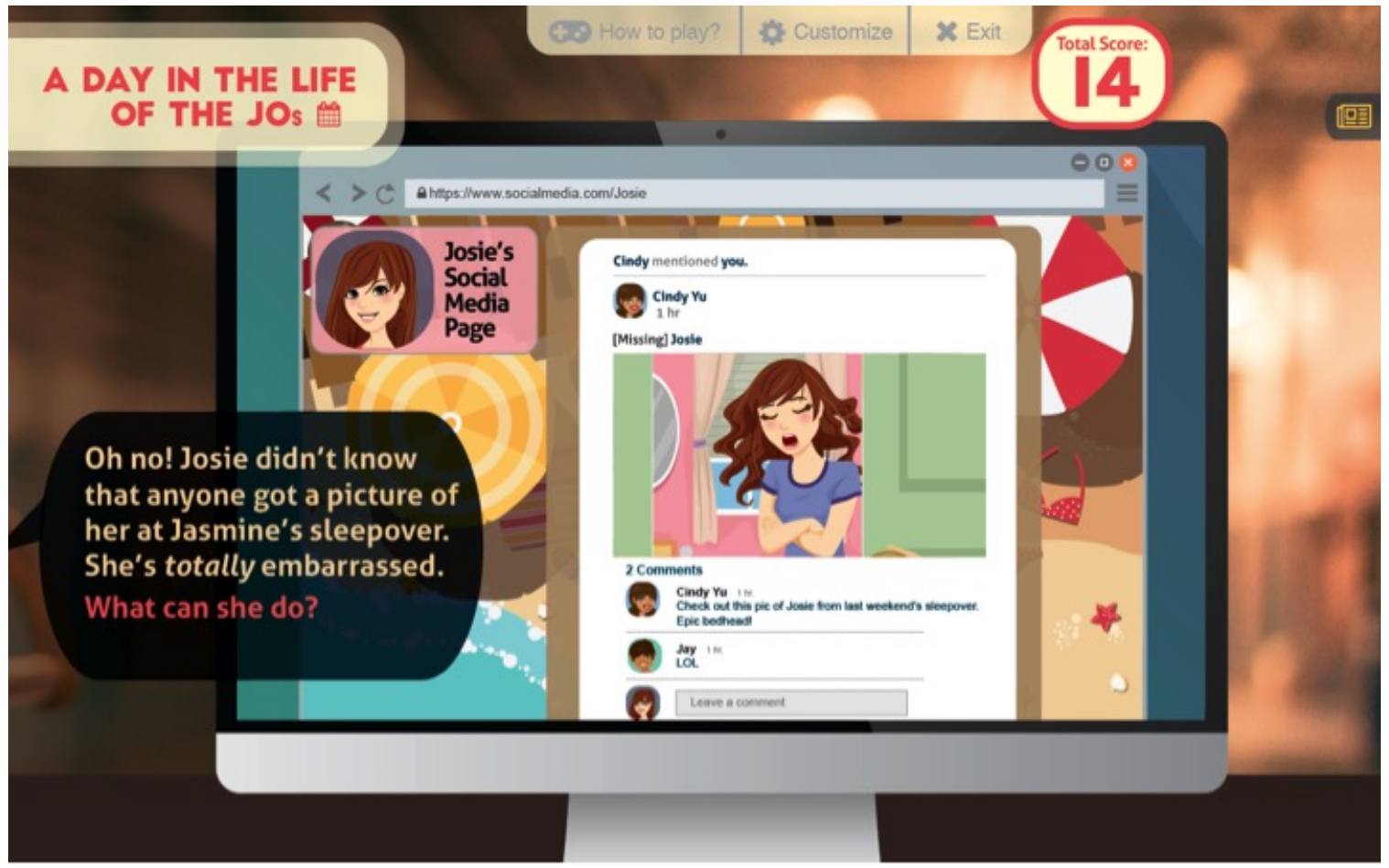

Figure A.45: D3-Internet privacy: Scene 2 of second scenario. 


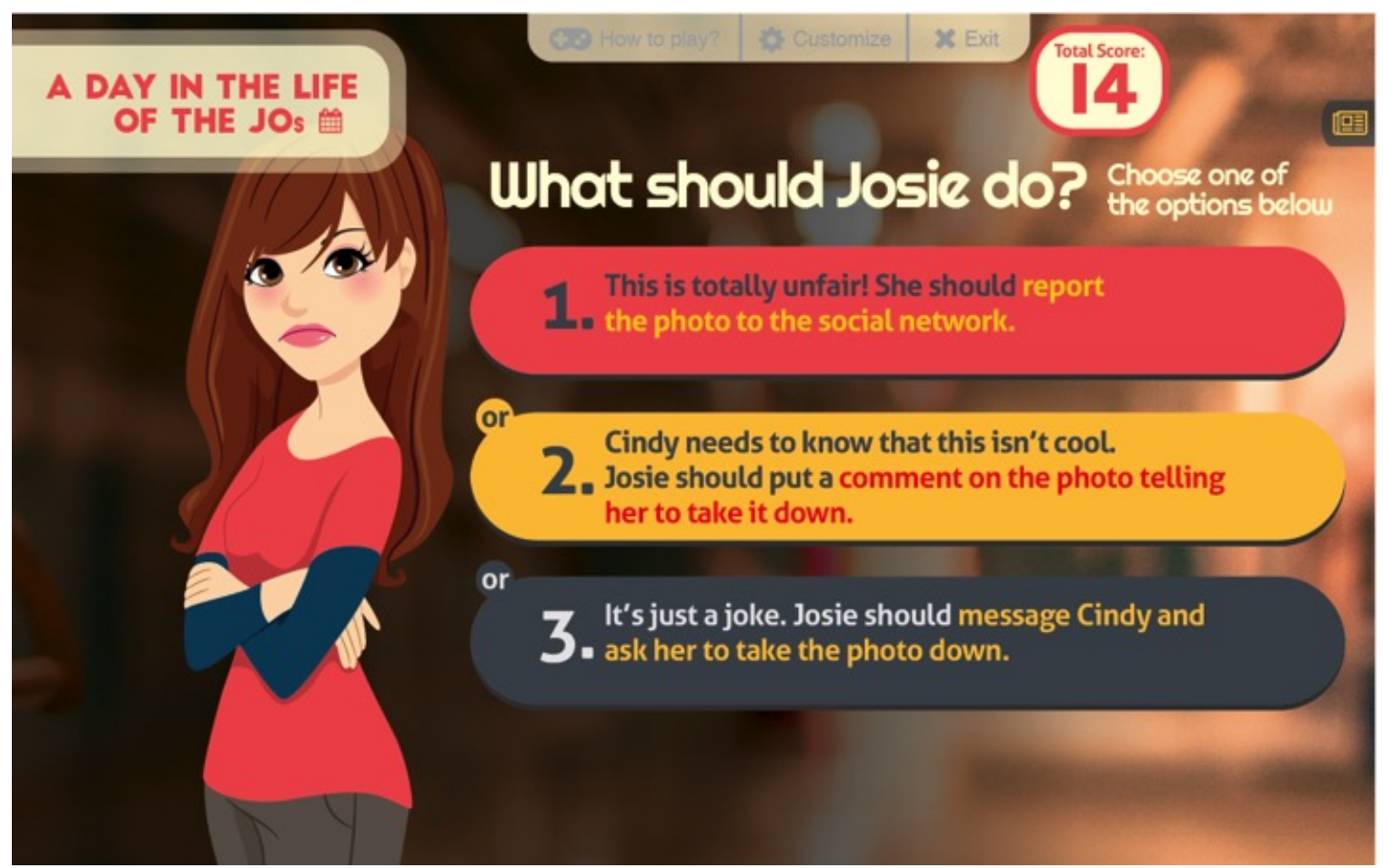

Figure A.46: D3-Internet privacy: Scene 3 of second scenario.

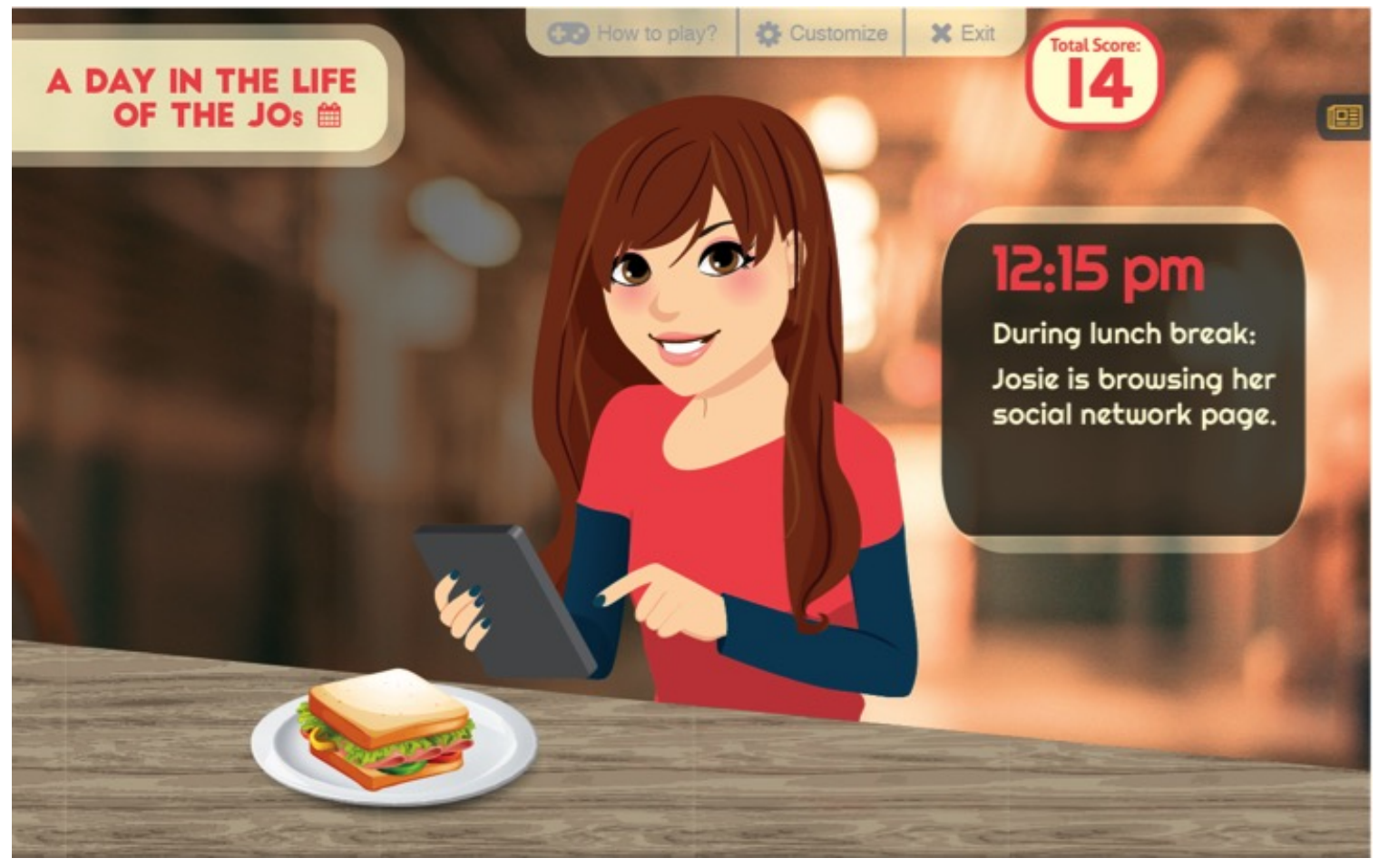

Figure A.47: D3-Internet privacy: Scene 1 of third scenario. 


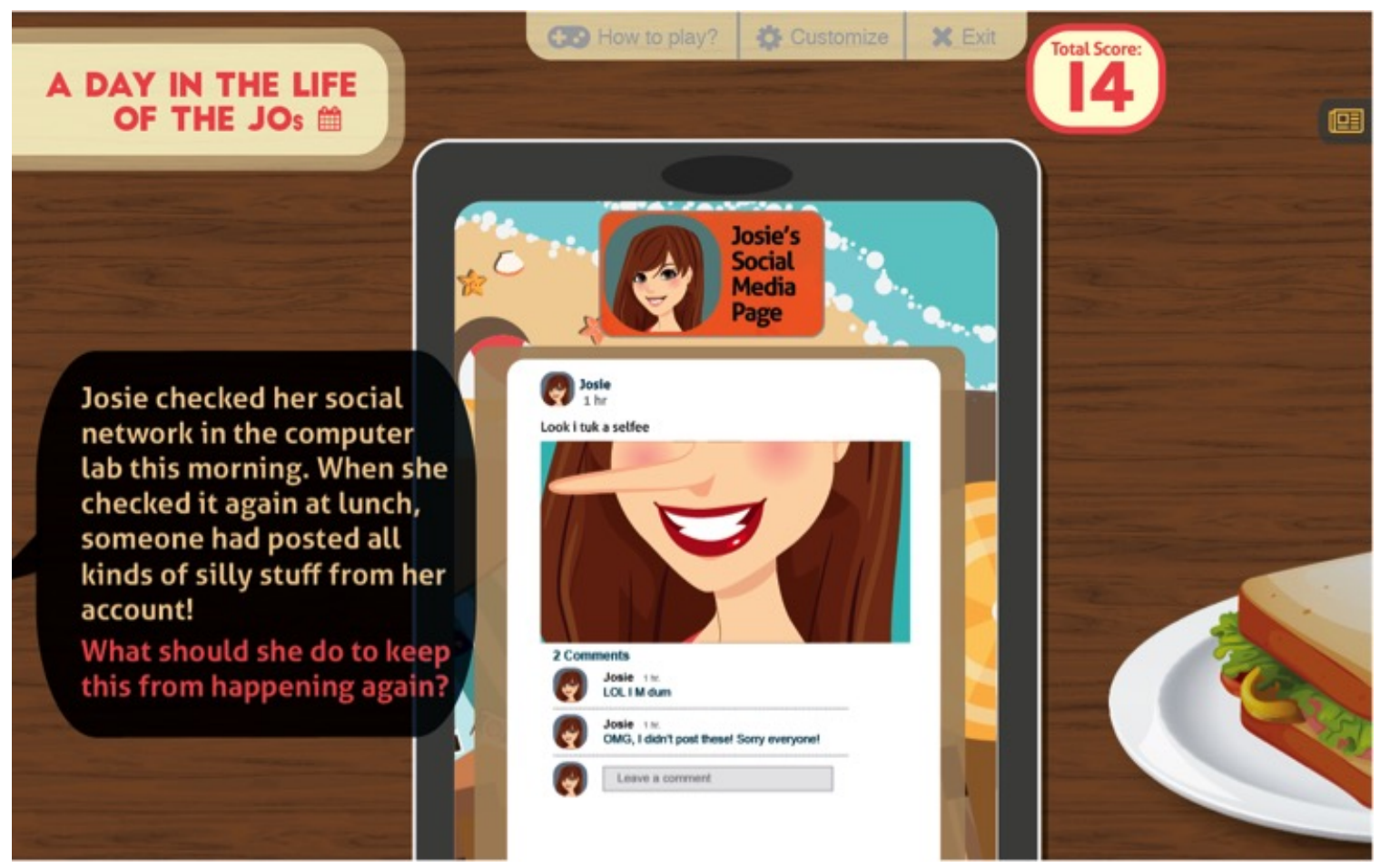

Figure A.48: D3-Internet privacy: Scene 2 of third scenario.

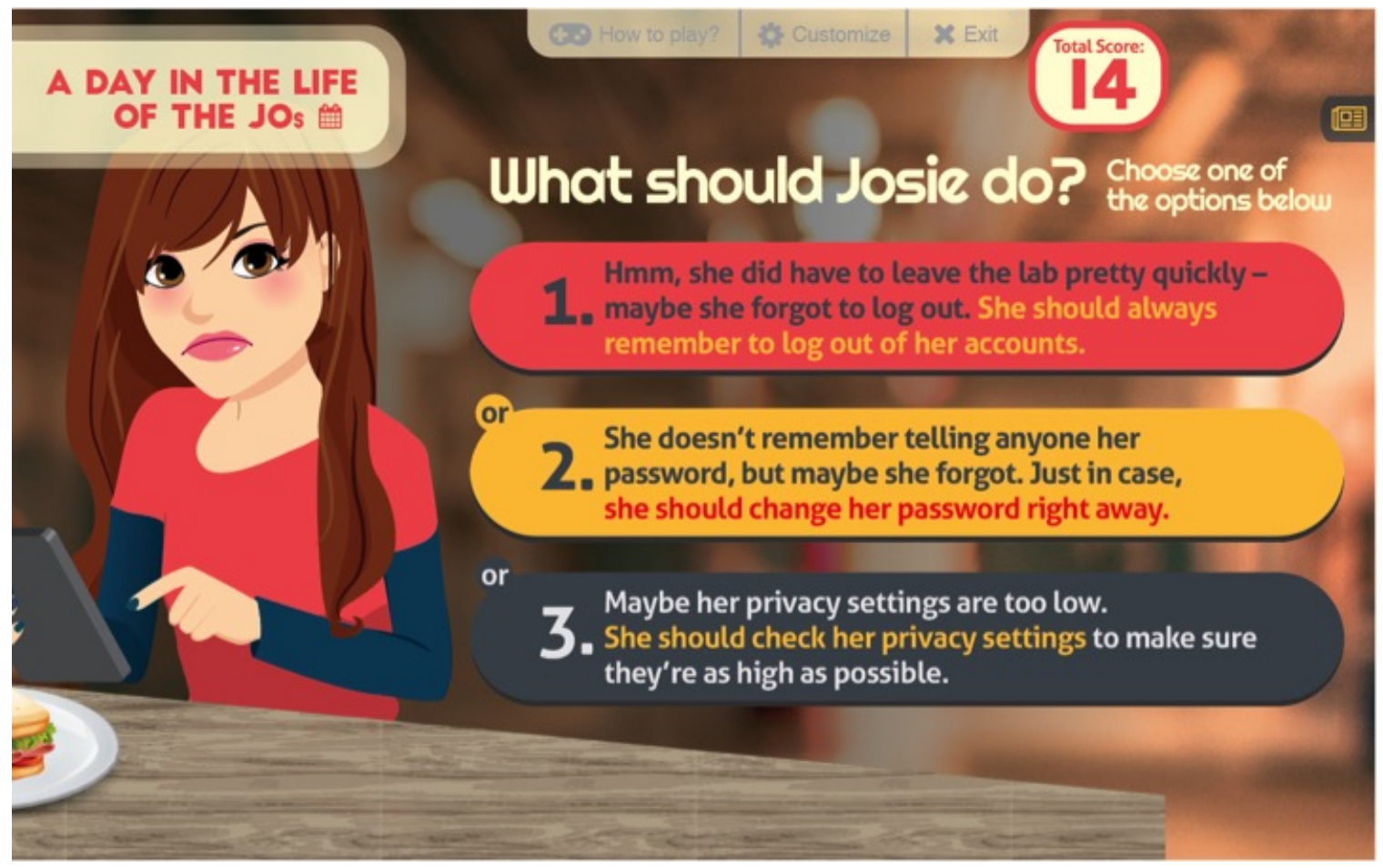

Figure A.49: D3-Internet privacy: Scene 3 of third scenario. 


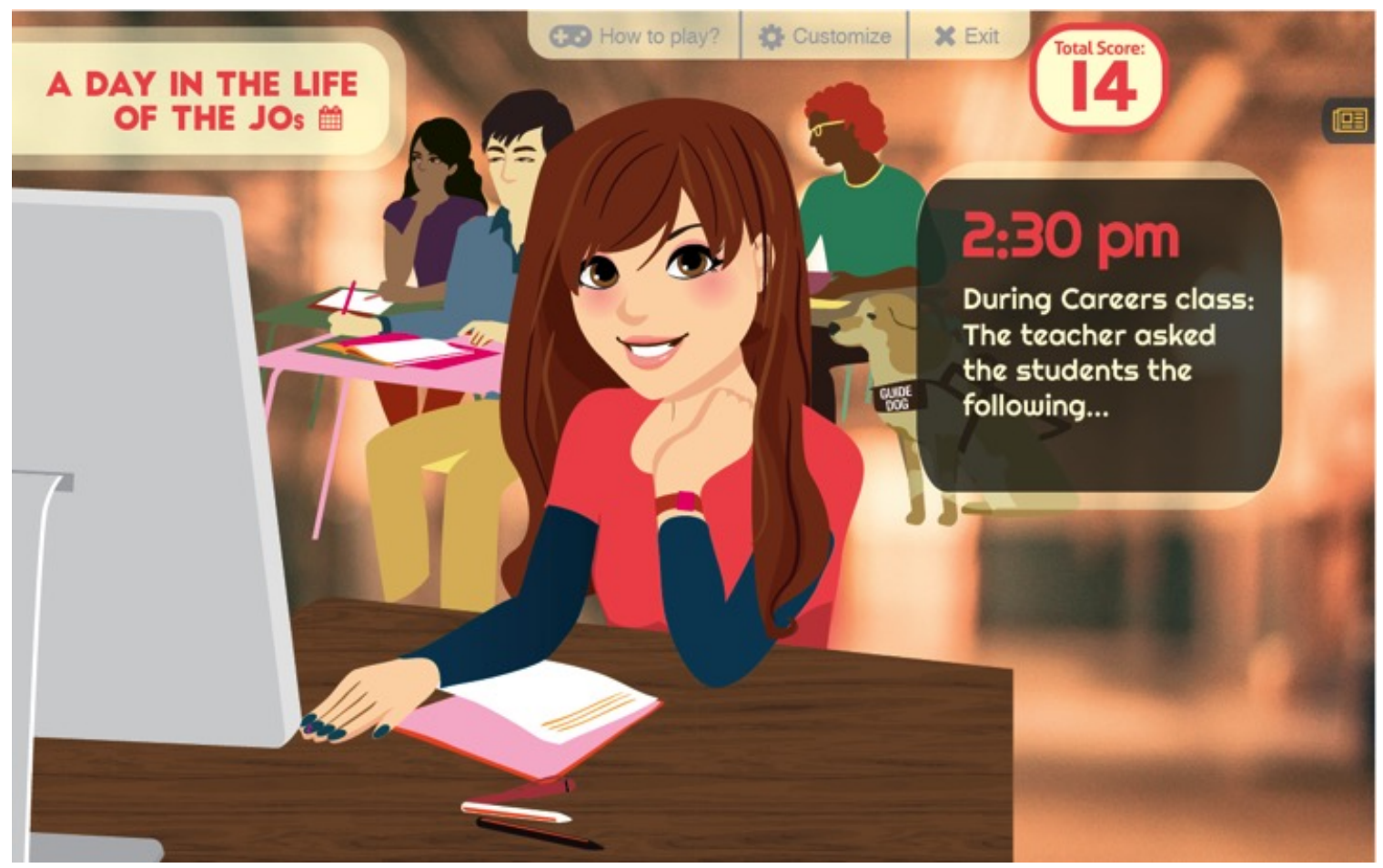

Figure A.50: D3-Internet privacy: Scene 1 of fourth scenario.

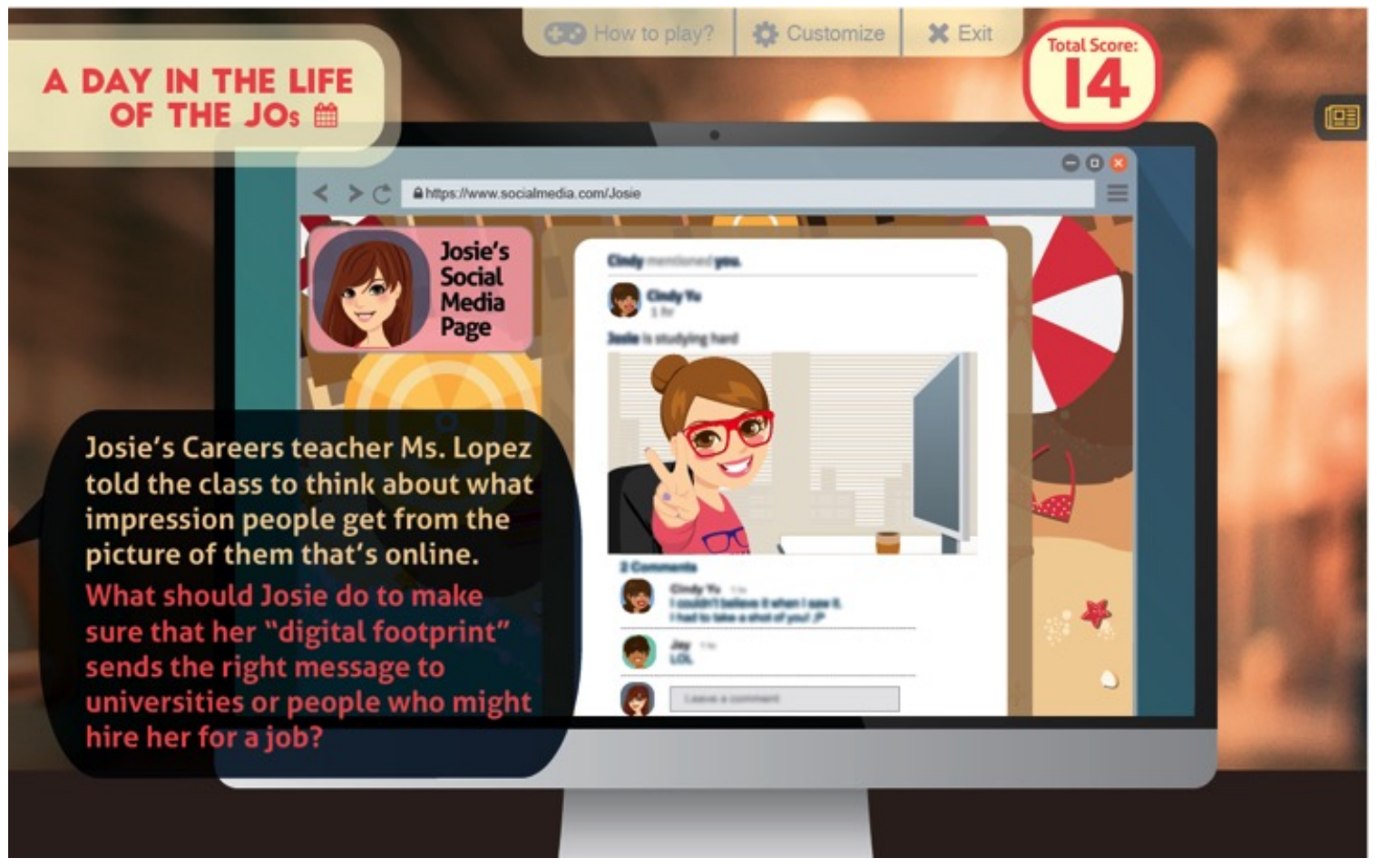

Figure A.51: D3-Internet privacy: Scene 2 of fourth scenario. 


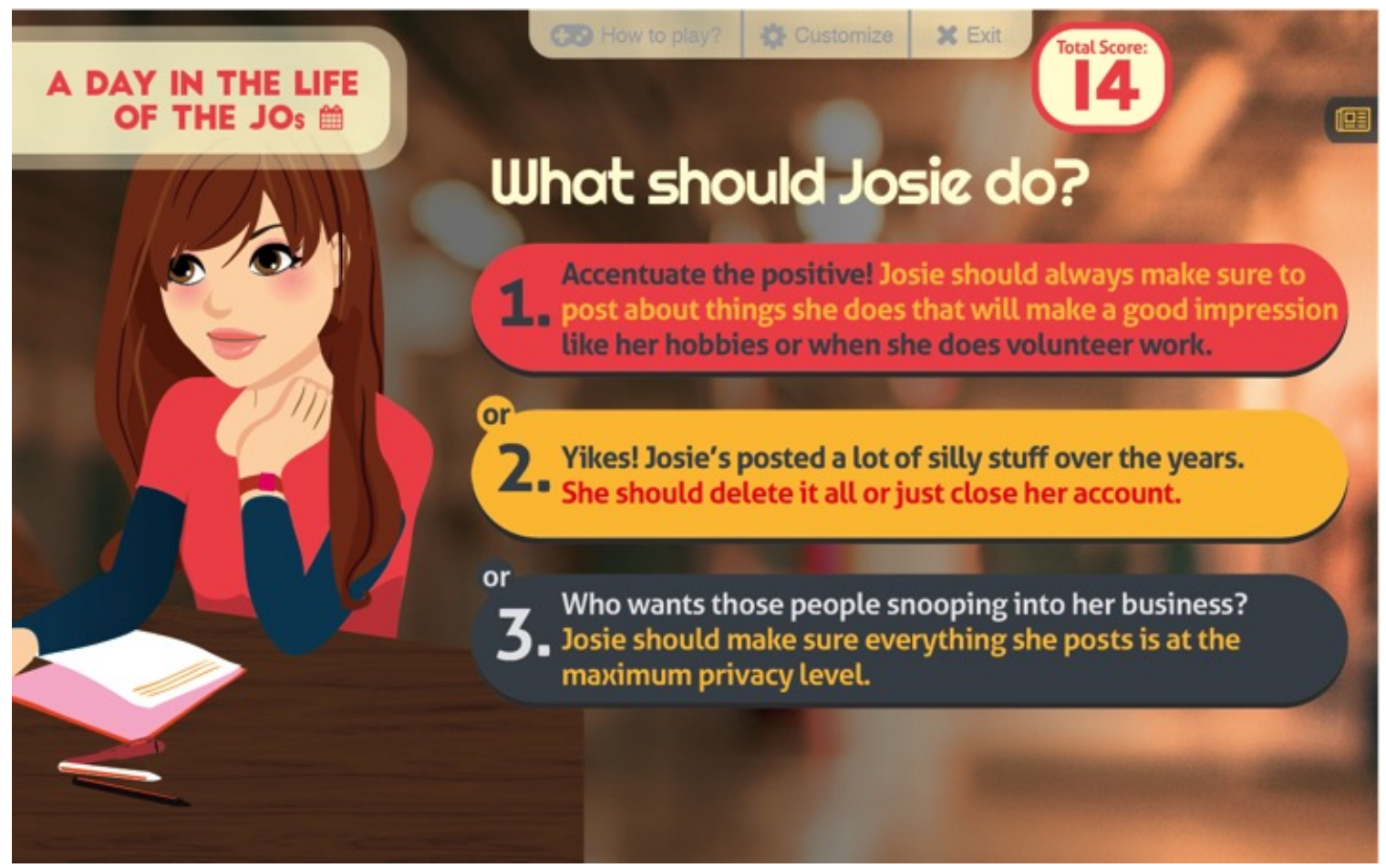

Figure A.52: D3-Internet privacy: Scene 3 of fourth scenario.

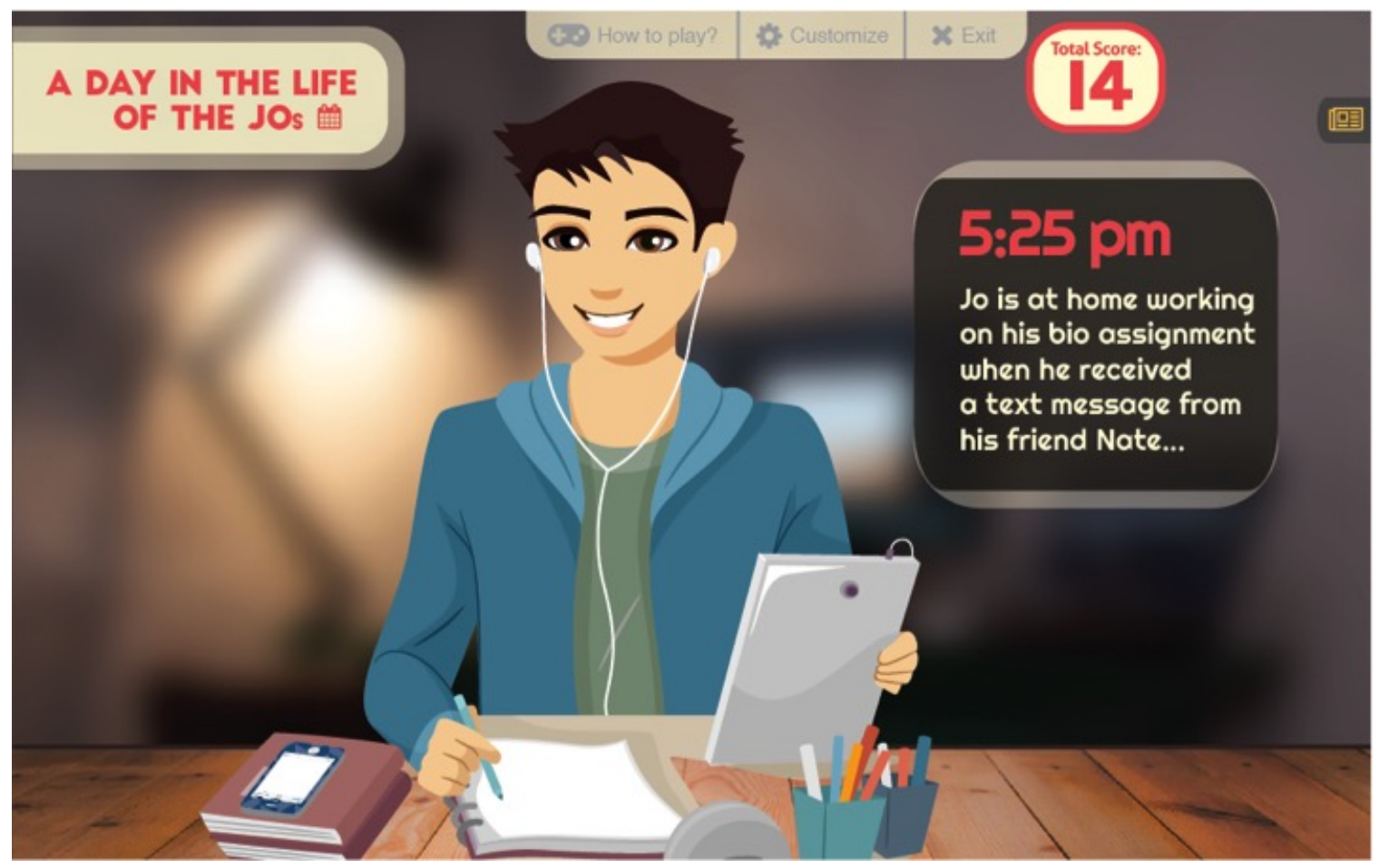

Figure A.53: D3-Internet privacy: Scene 1 of fifth scenario. 


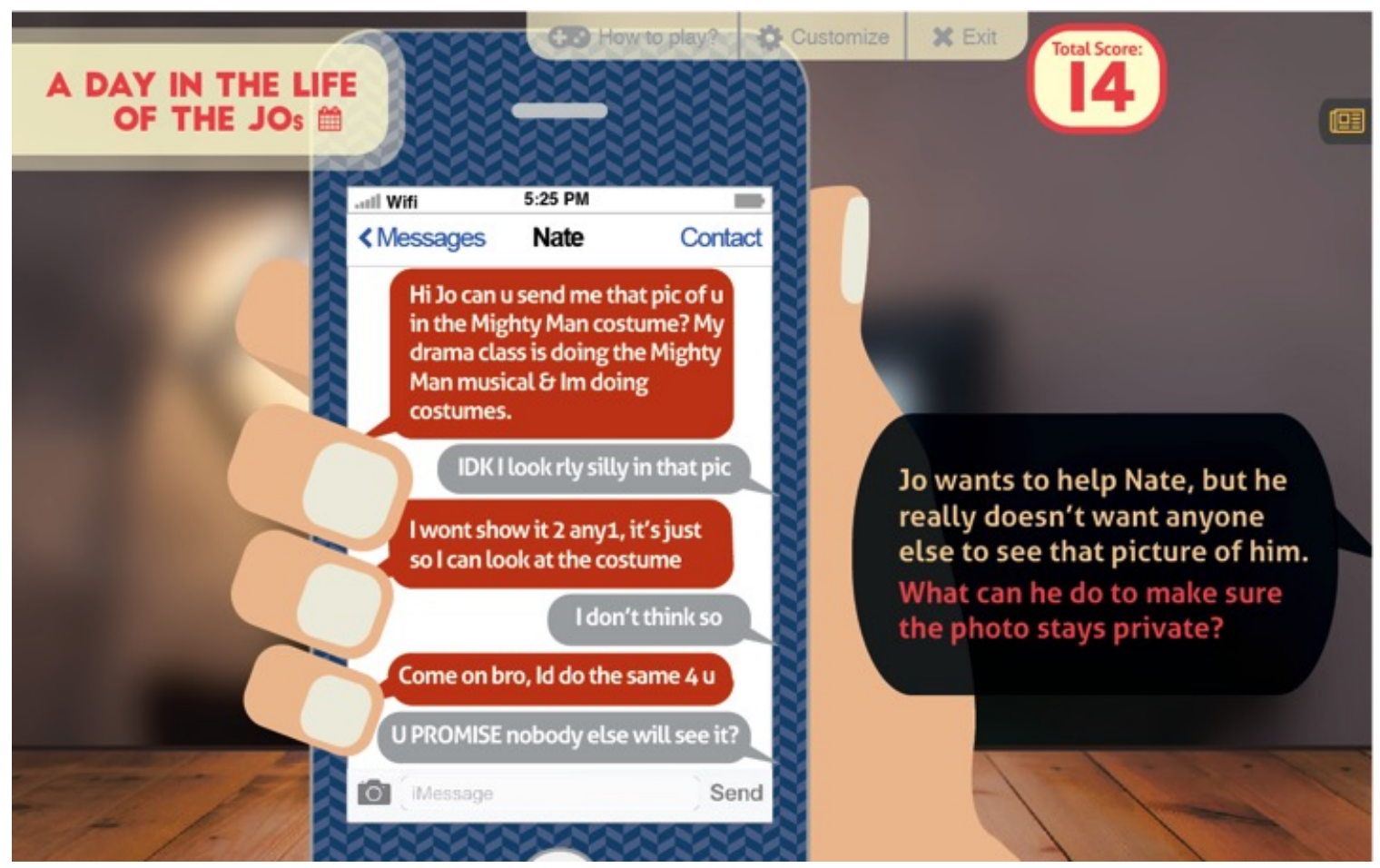

Figure A.54: D3-Internet privacy: Scene 2 of fifth scenario.

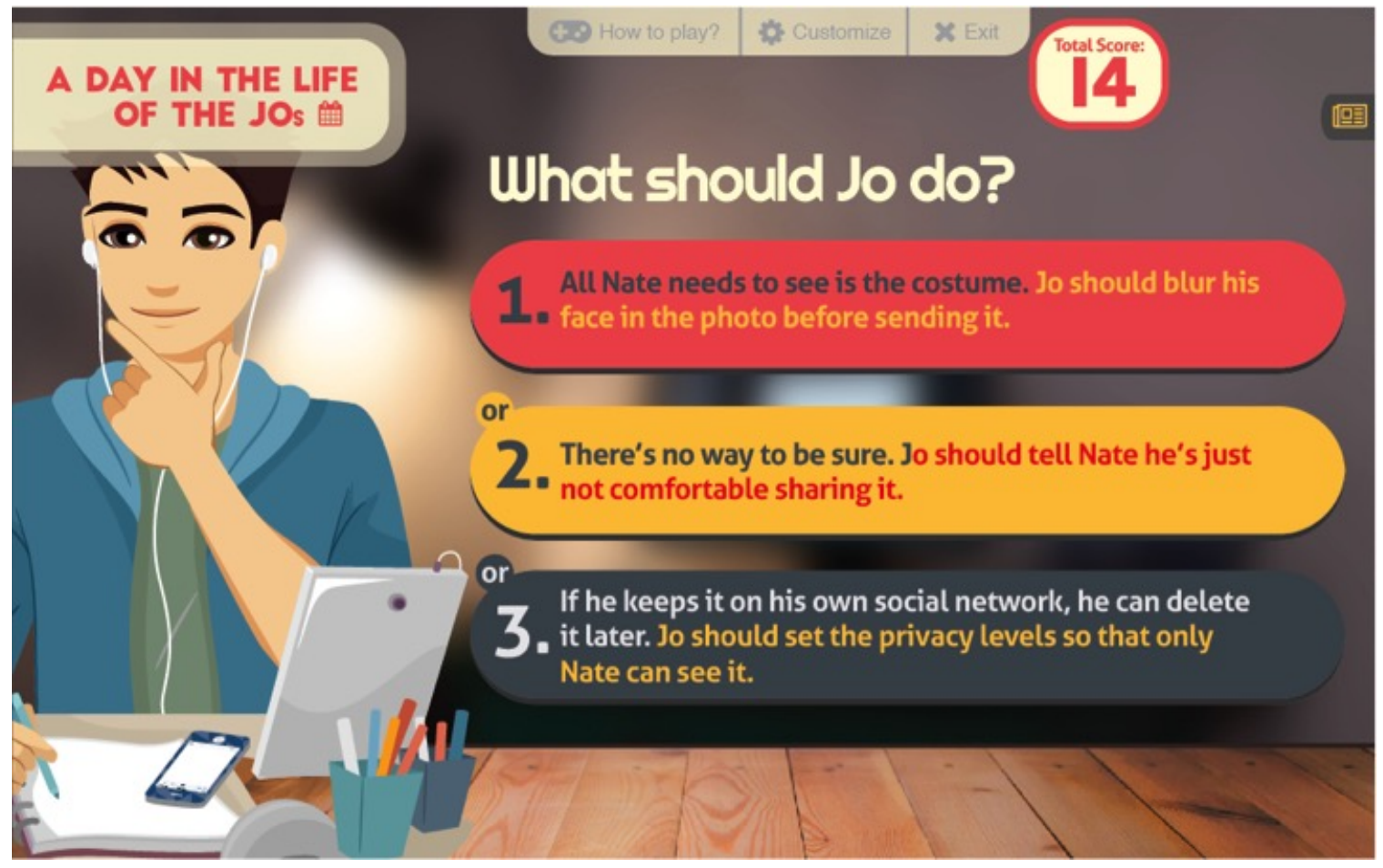

Figure A.55: D3-Internet privacy: Scene 3 of fifth scenario. 


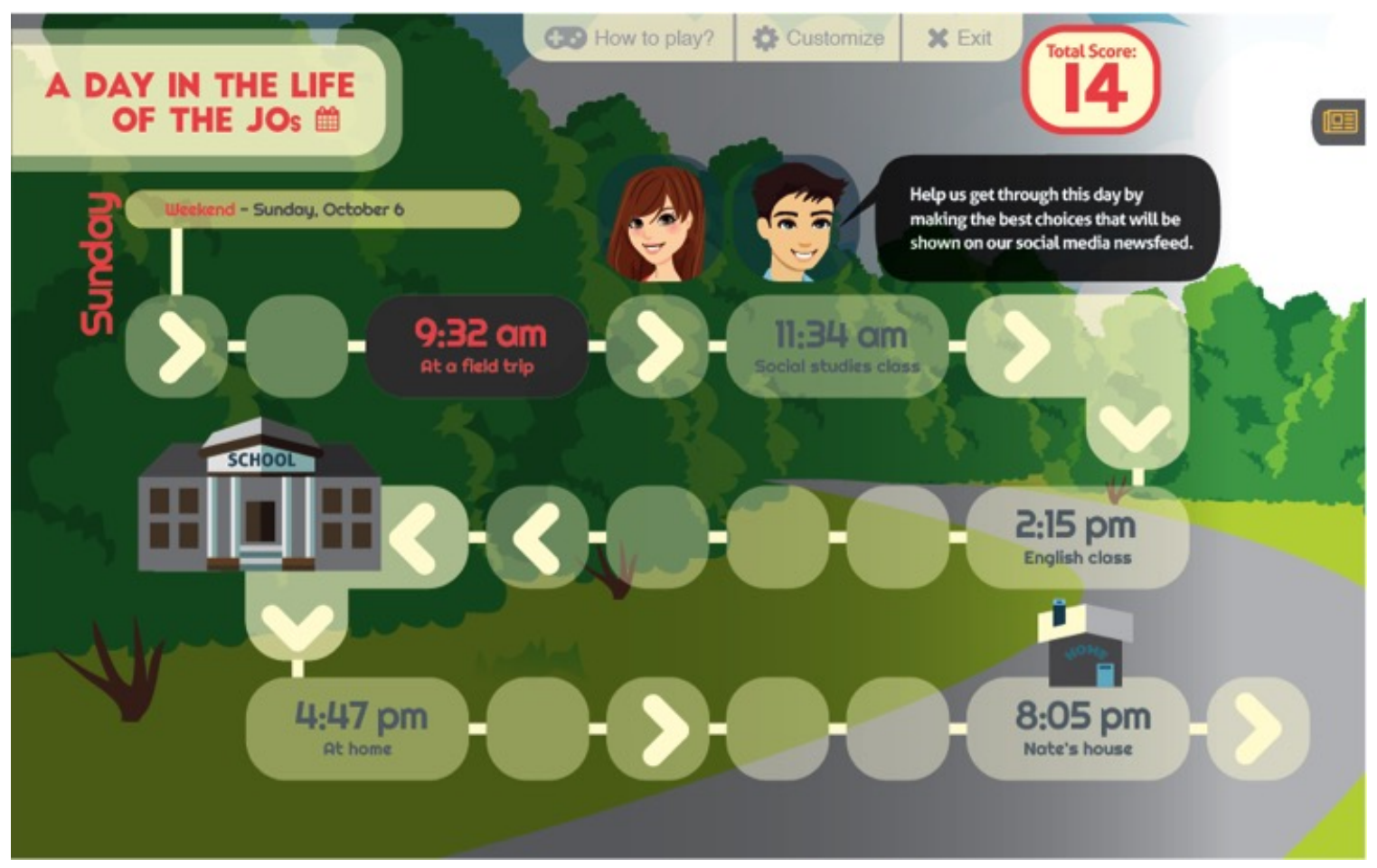

Figure A.56: The Day View of D4-Image sharing topic.

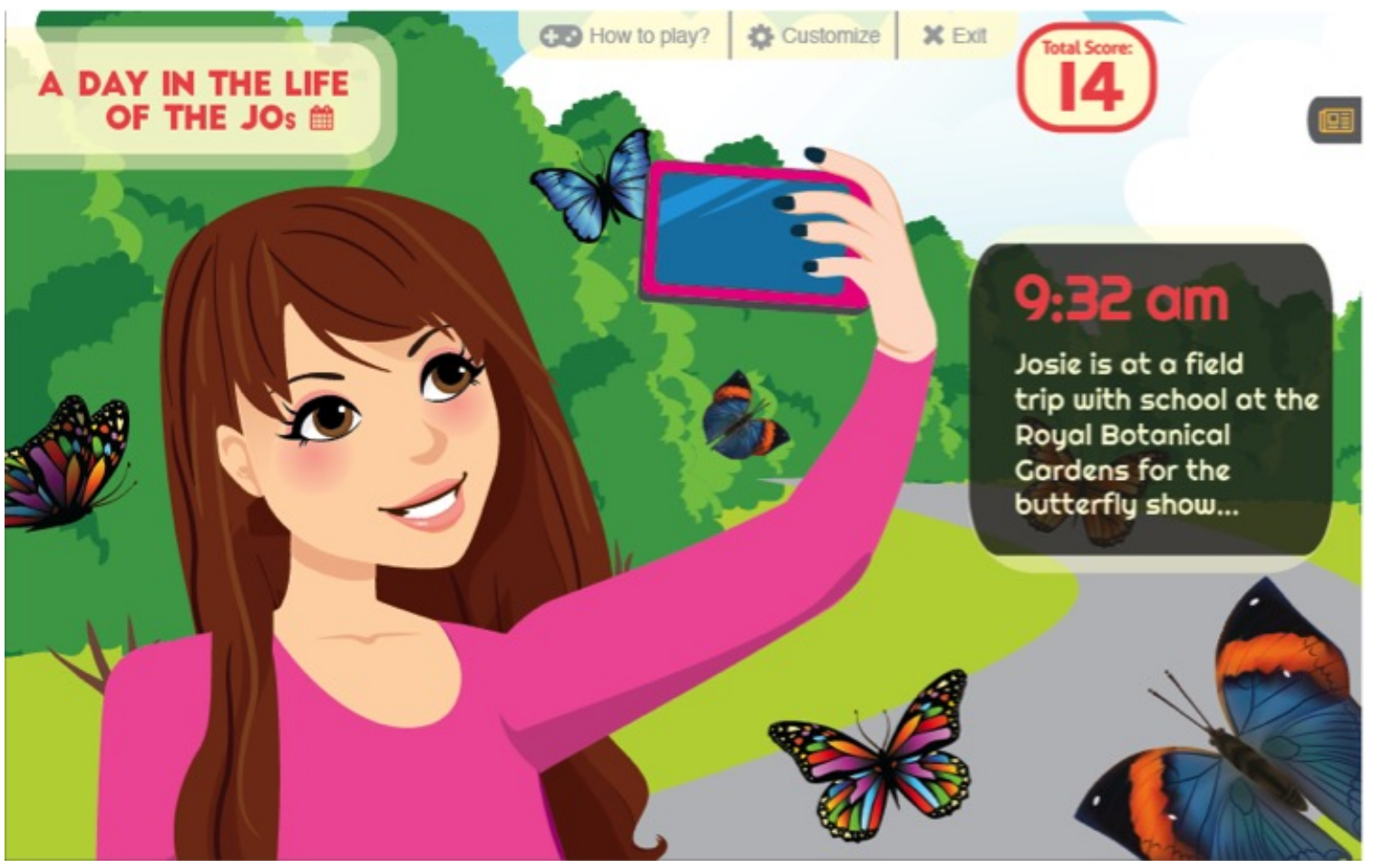

Figure A.57: D4-Image sharing: Scene 1 of first scenario. 


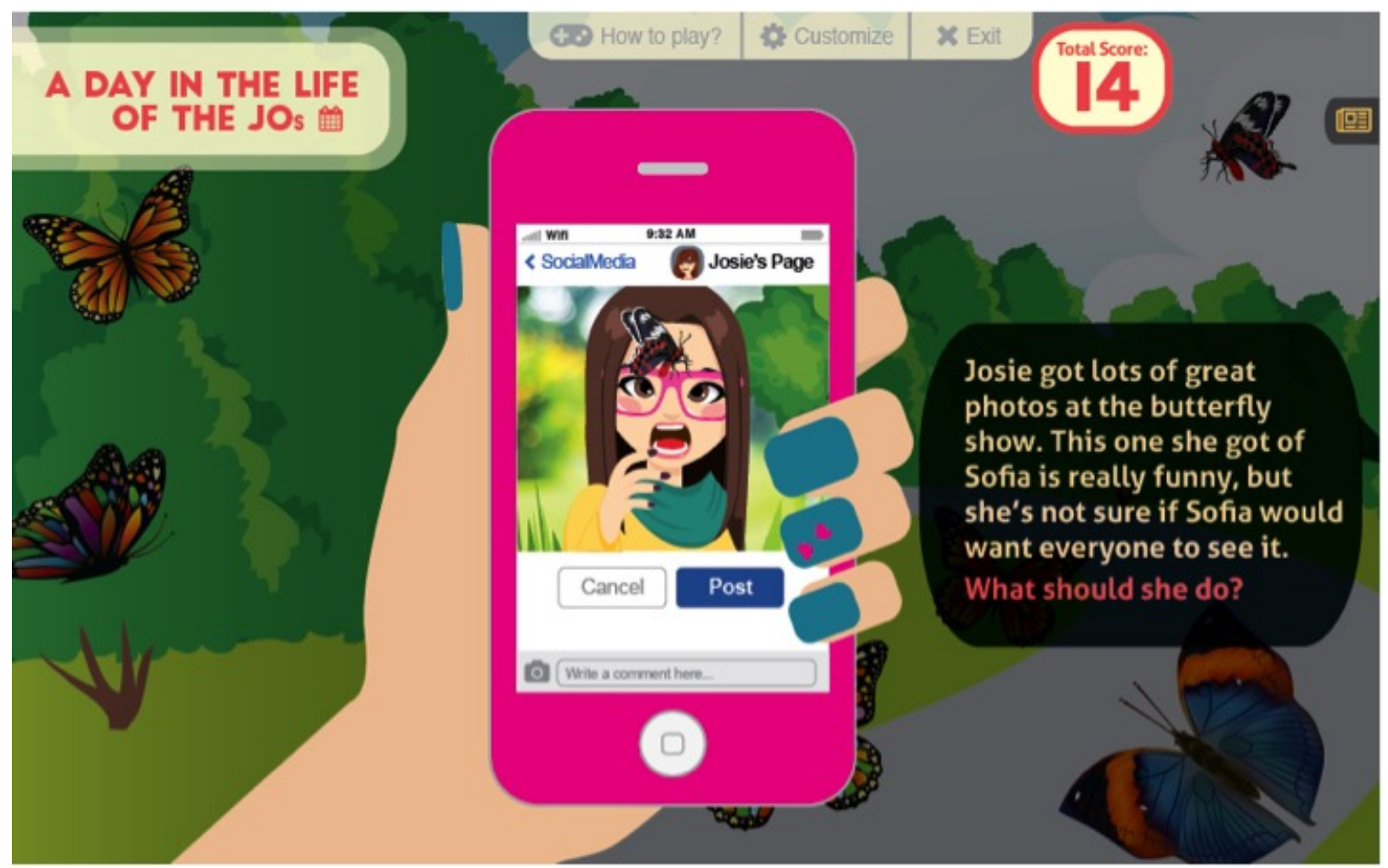

Figure A.58: D4-Image sharing: Scene 2 of first scenario.

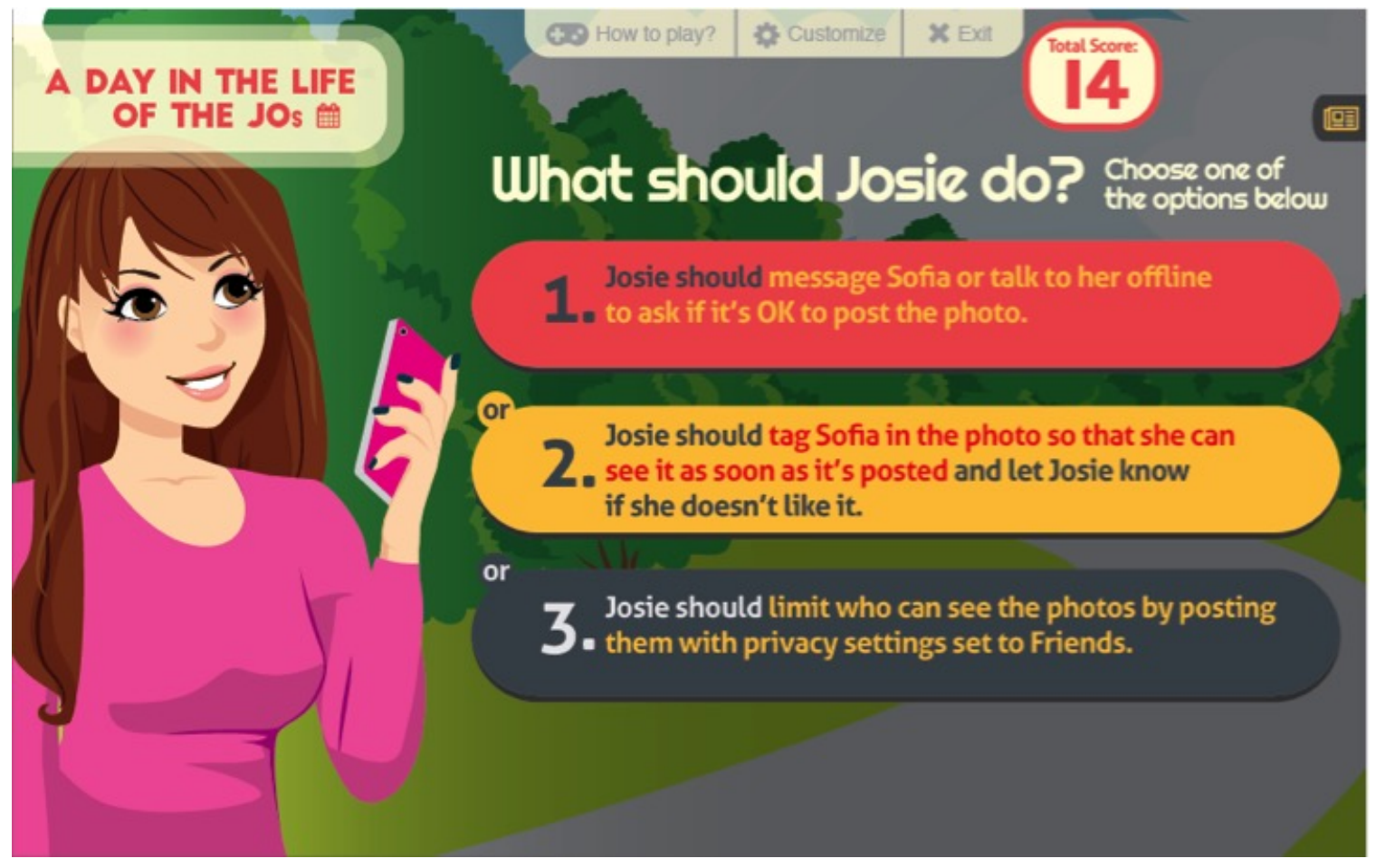

Figure A.59: D4-Image sharing: Scene 3 of first scenario. 


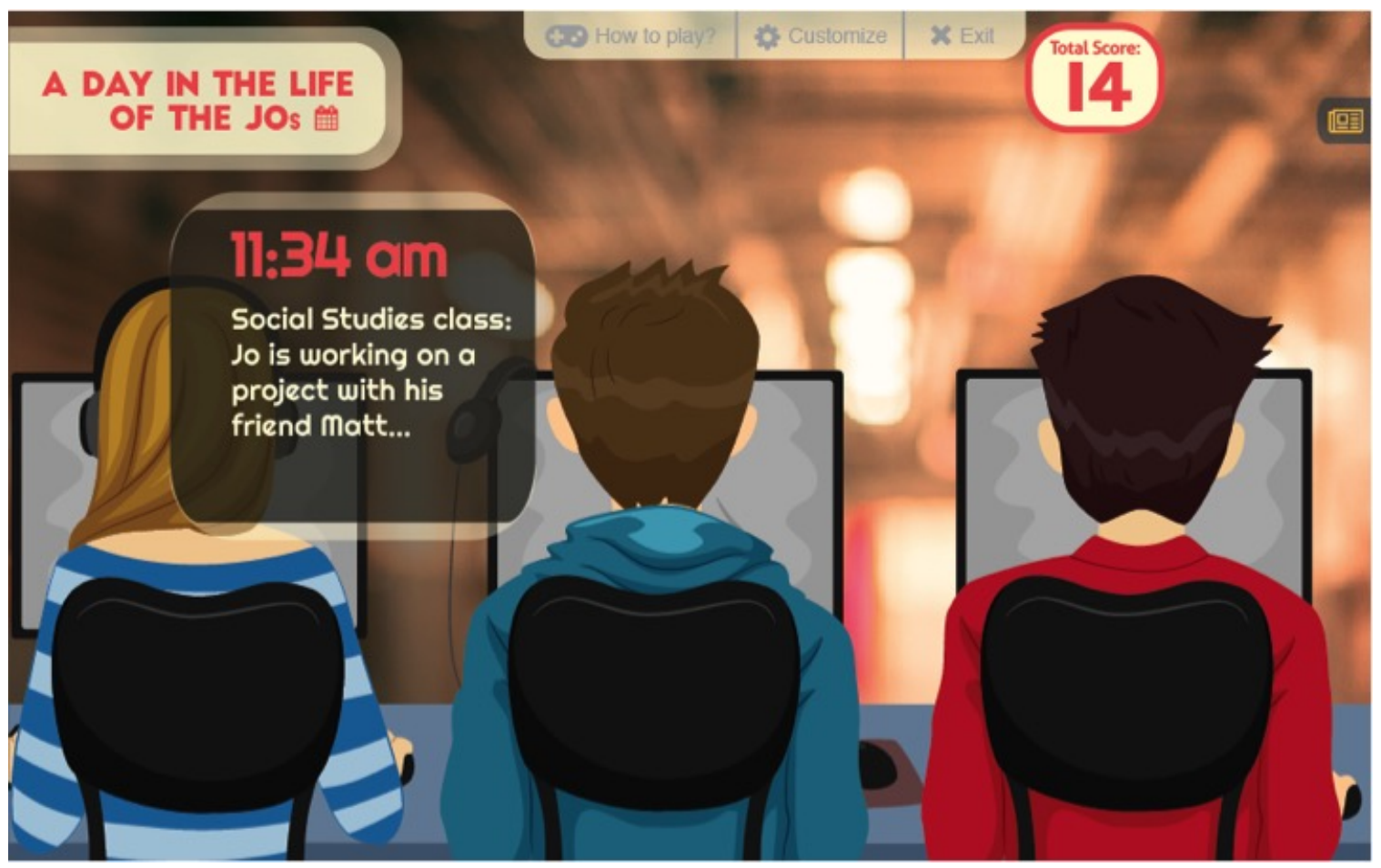

Figure A.60: D4-Image sharing: Scene 1 of second scenario.

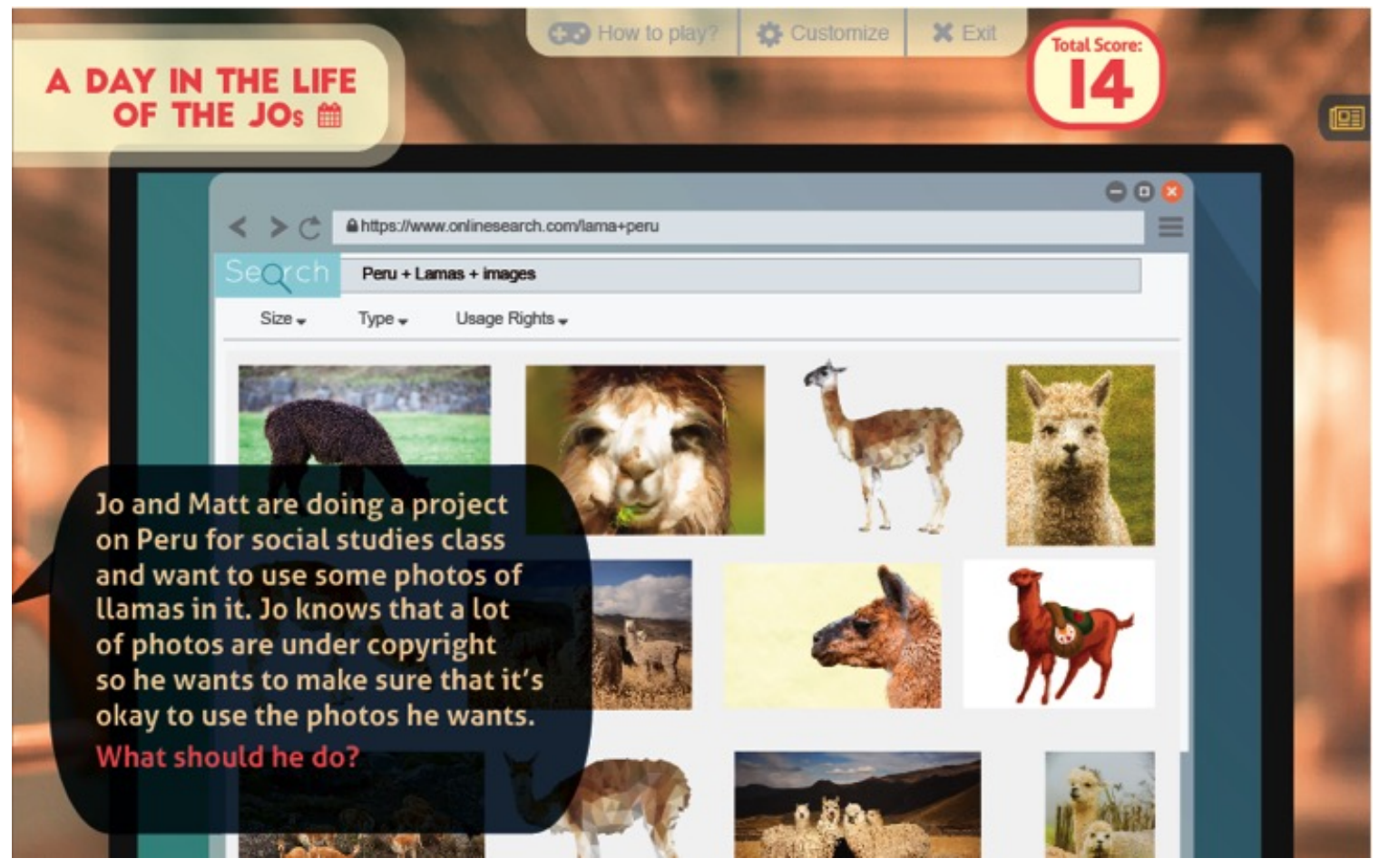

Figure A.61: D4-Image sharing: Scene 2 of second scenario. 


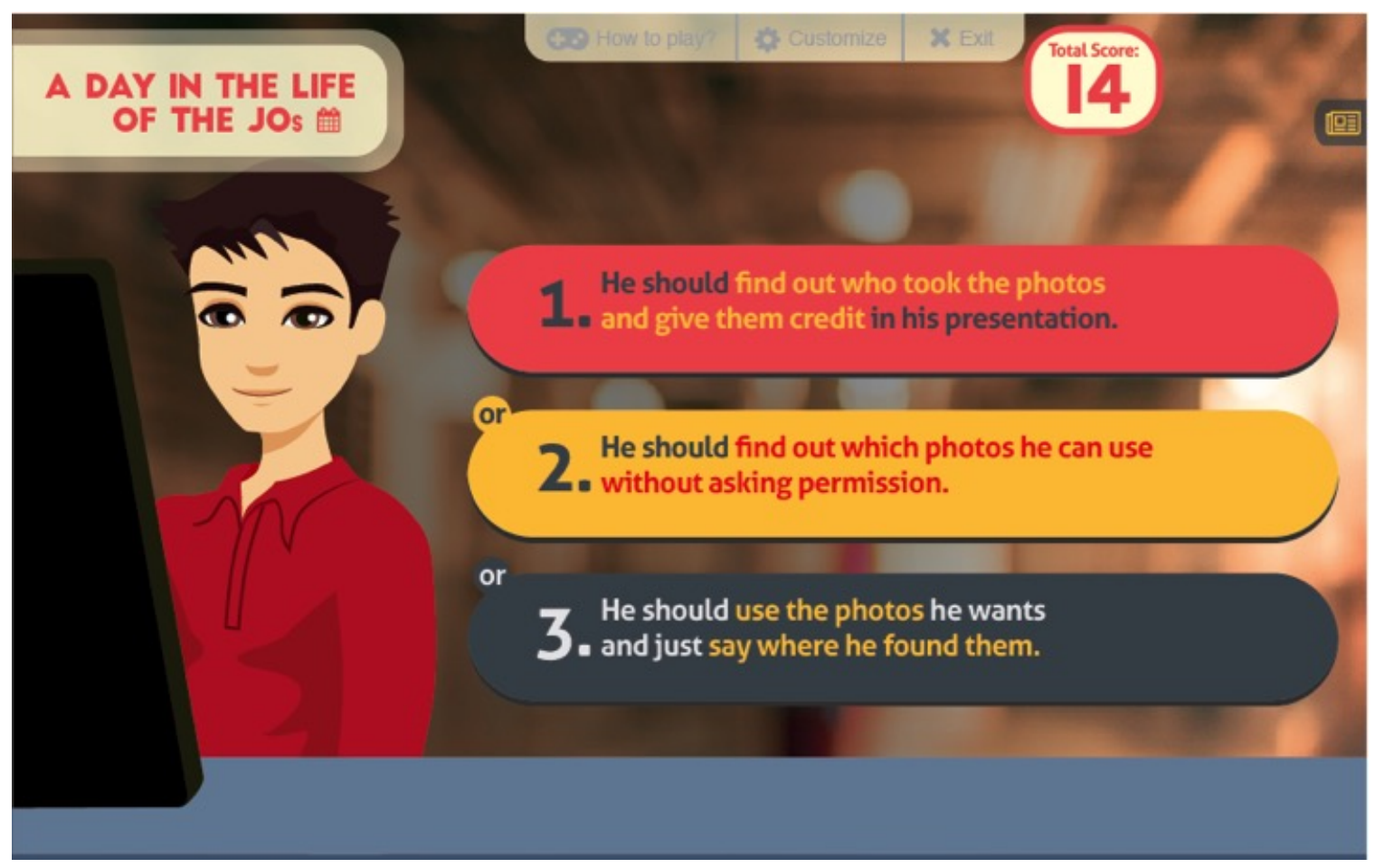

Figure A.62: D4-Image sharing: Scene 3 of second scenario.

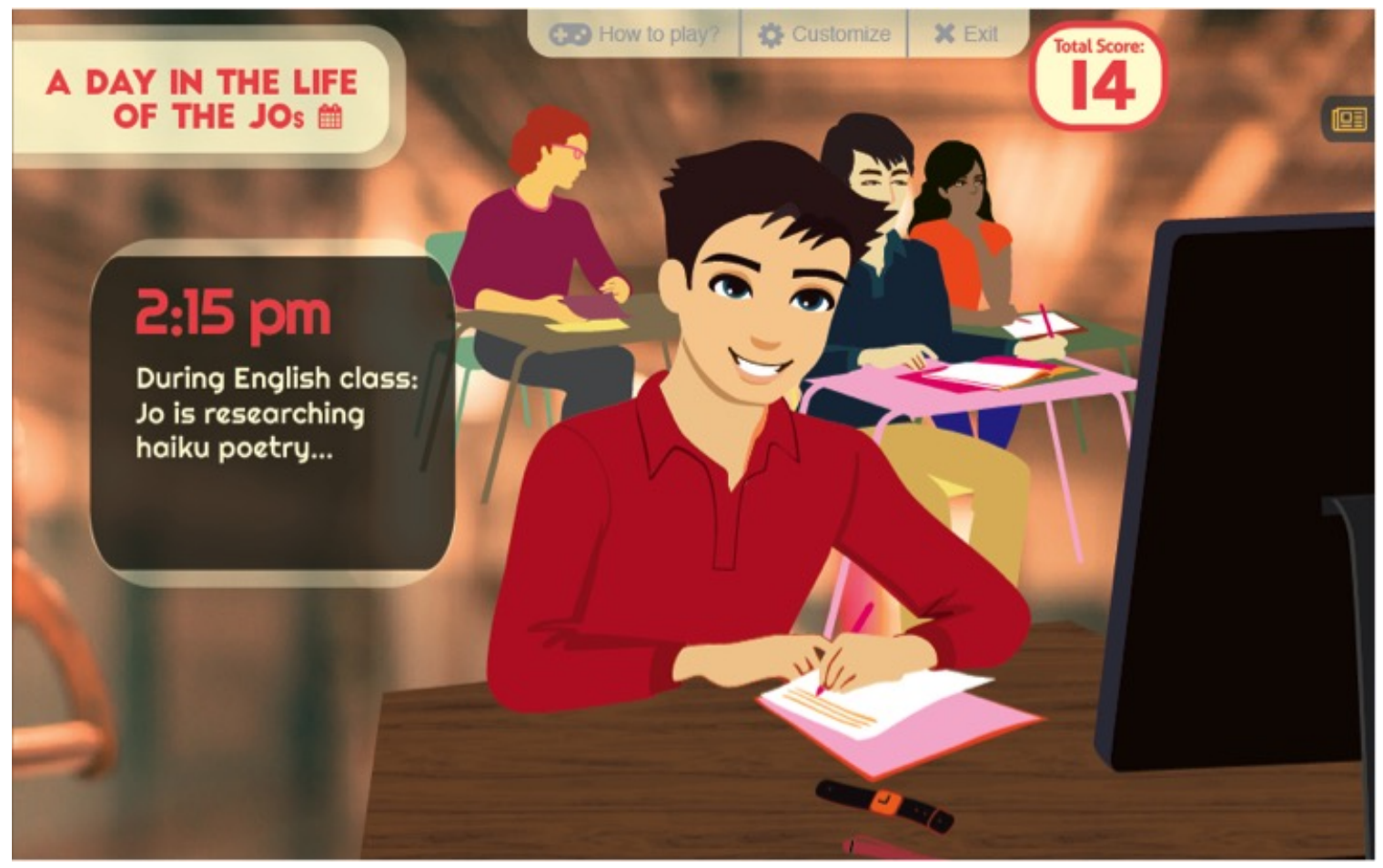

Figure A.63: D4-Image sharing: Scene 1 of third scenario. 


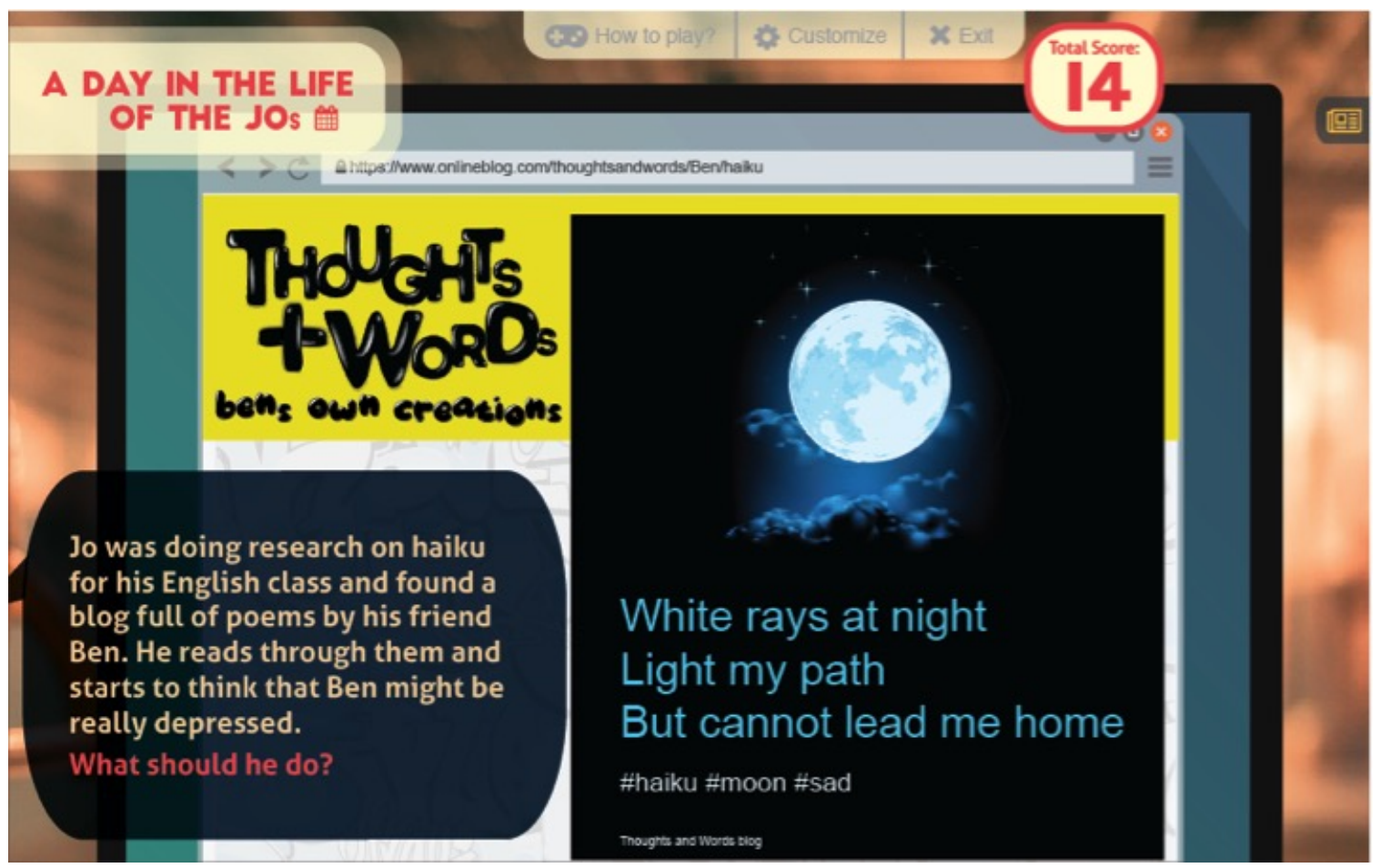

Figure A.64: D4-Image sharing: Scene 2 of third scenario.

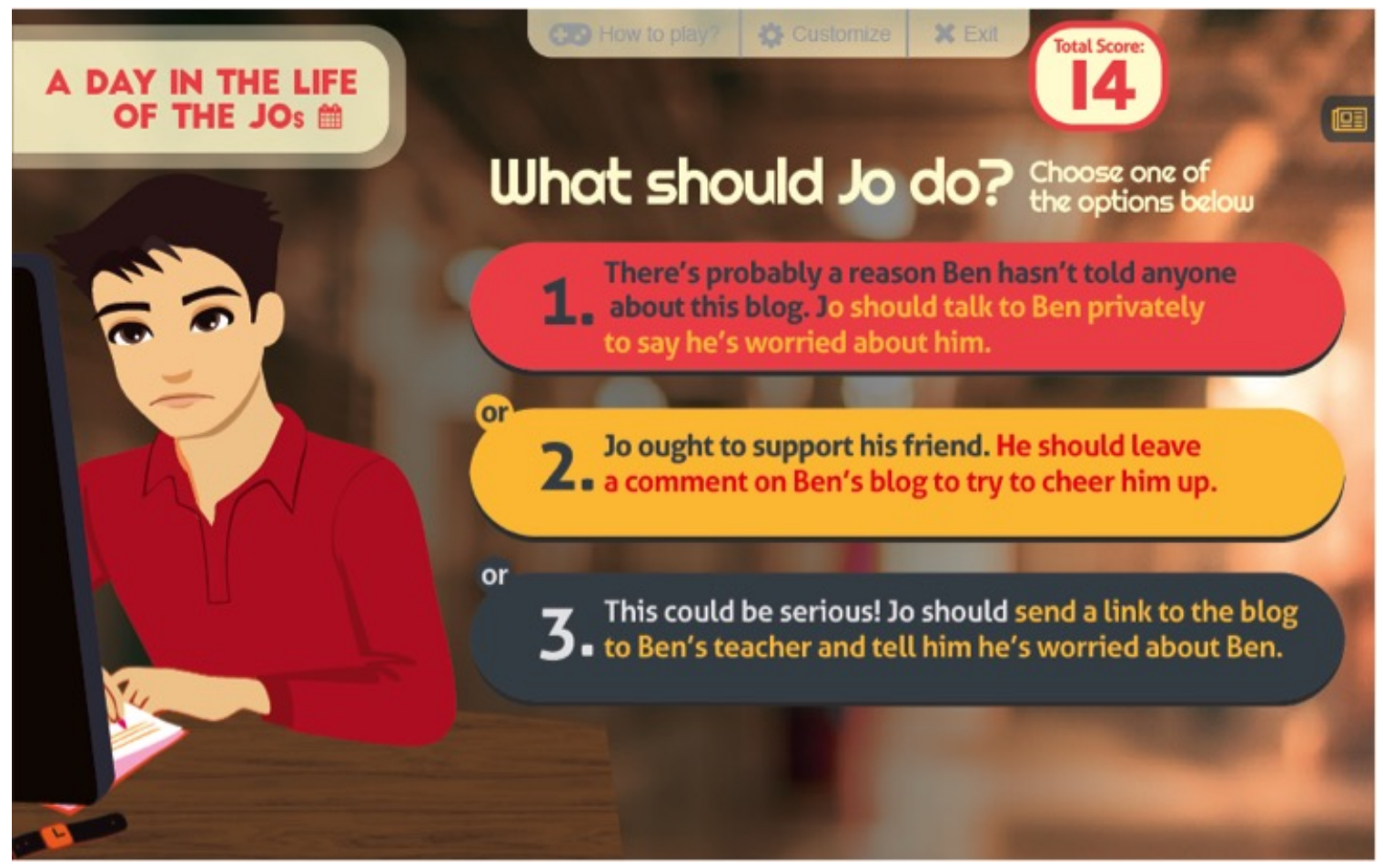

Figure A.65: D4-Image sharing: Scene 3 of third scenario. 


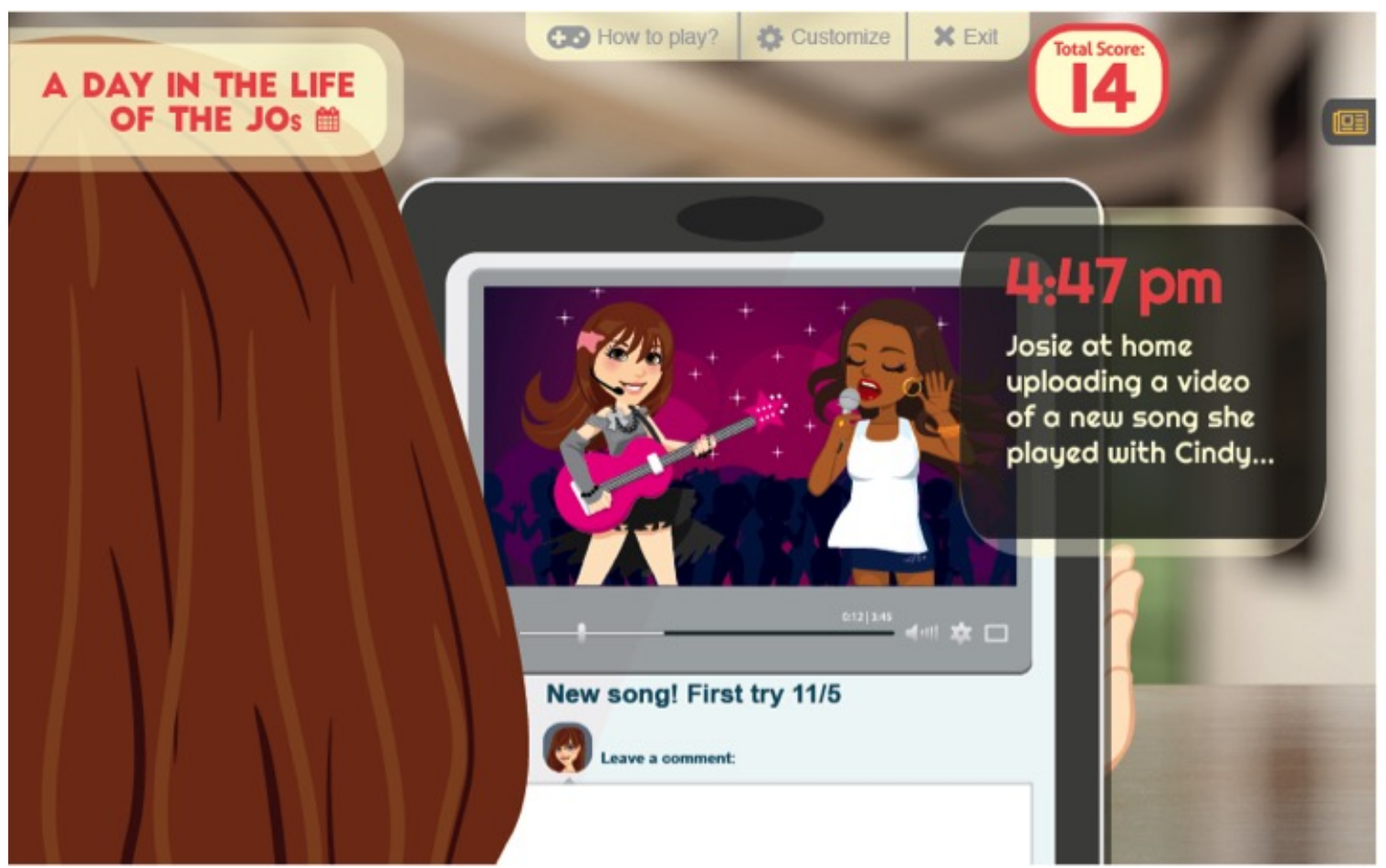

Figure A.66: D4-Image sharing: Scene 1 of fourth scenario.

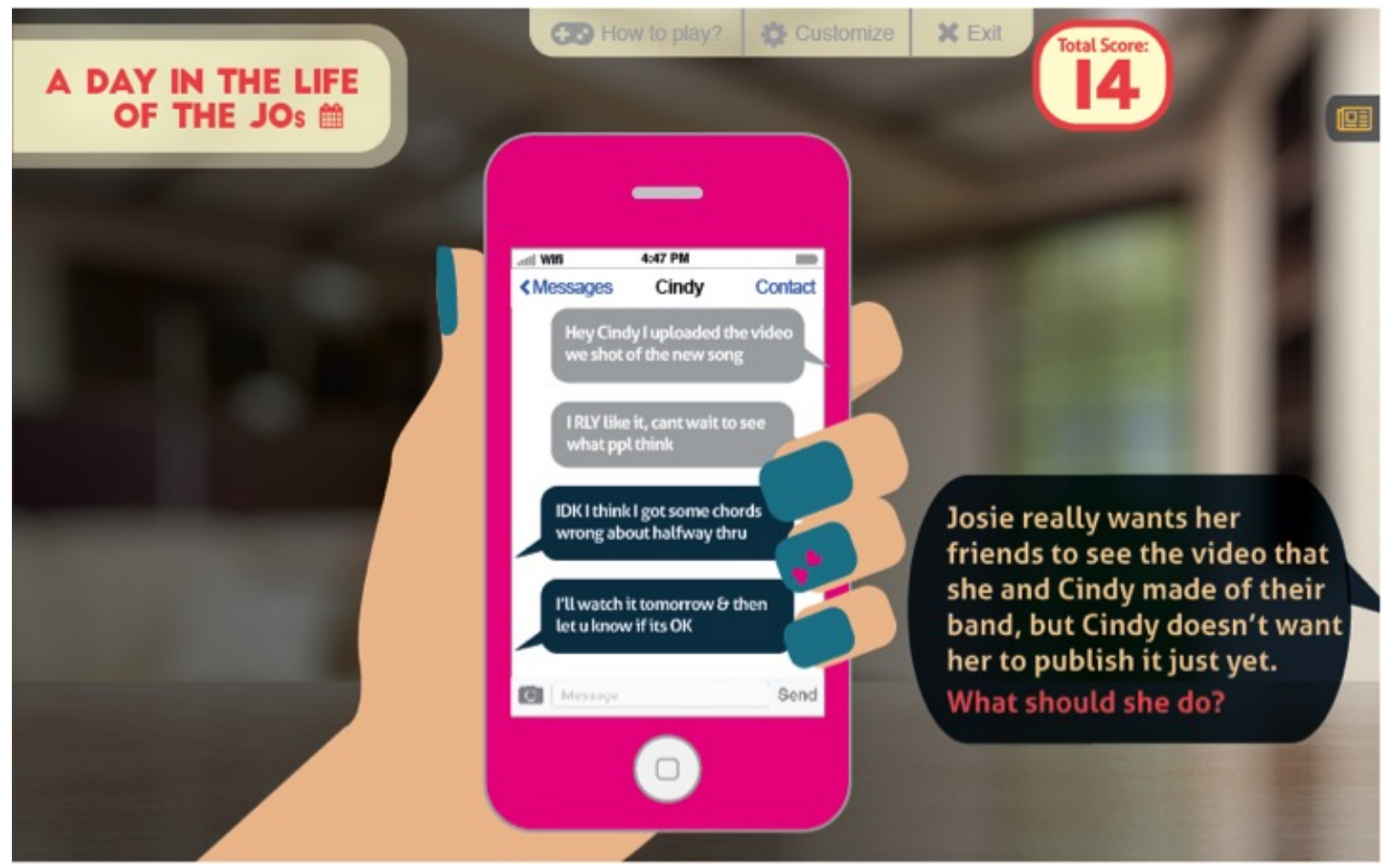

Figure A.67: D4-Image sharing: Scene 2 of fourth scenario. 


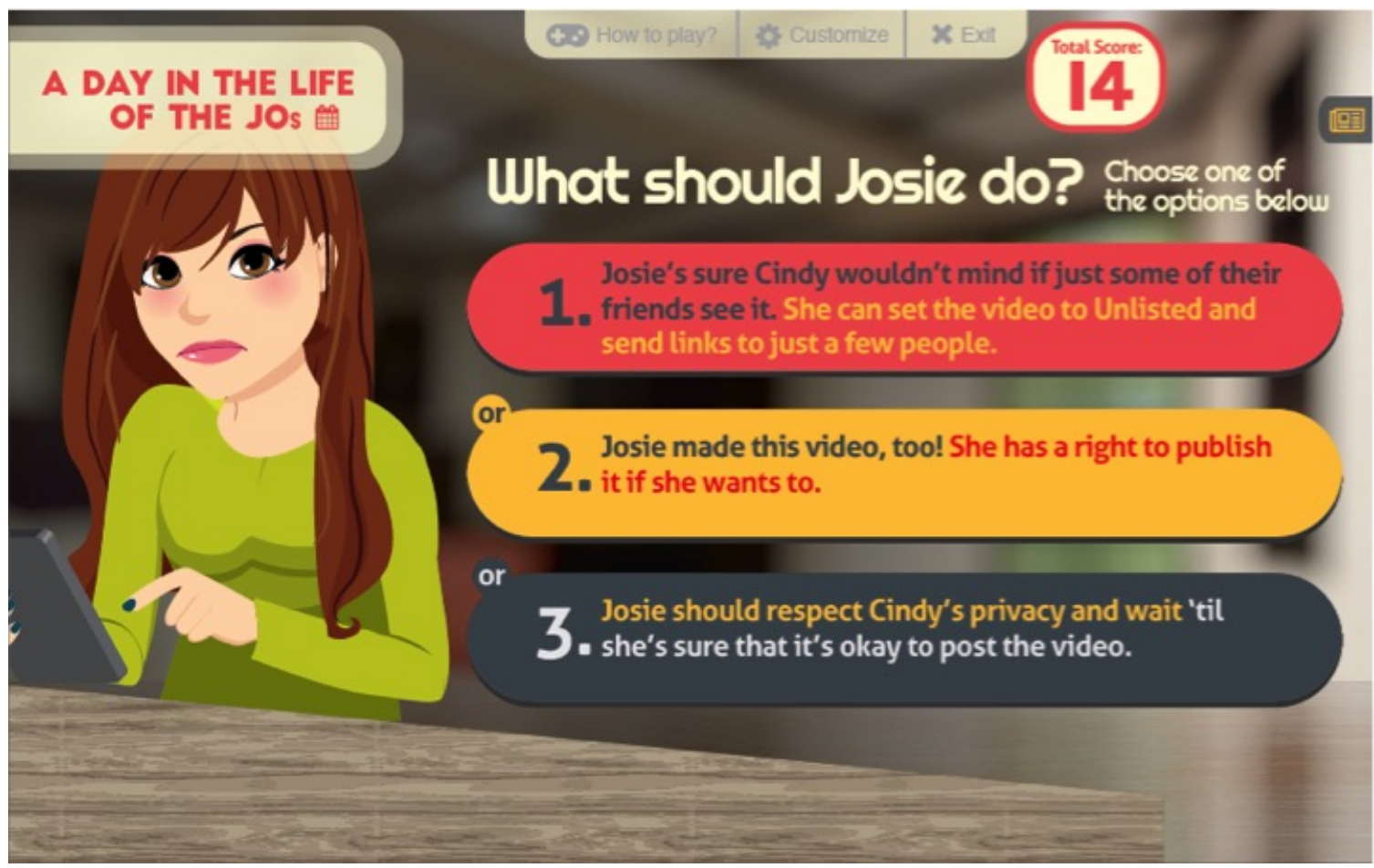

Figure A.68: D4-Image sharing: Scene 3 of fourth scenario.

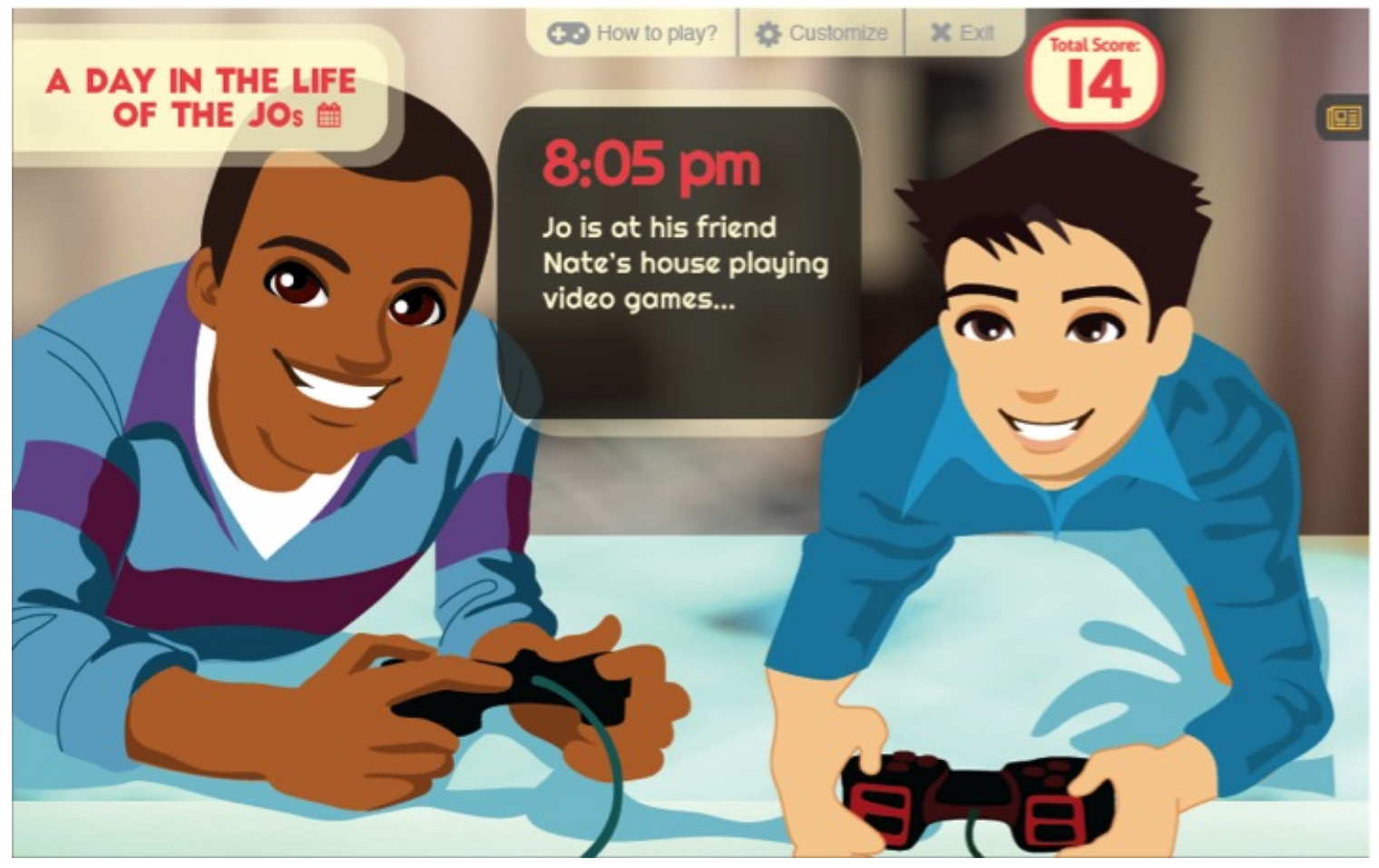

Figure A.69: D4-Image sharing: Scene 1 of fifth scenario. 


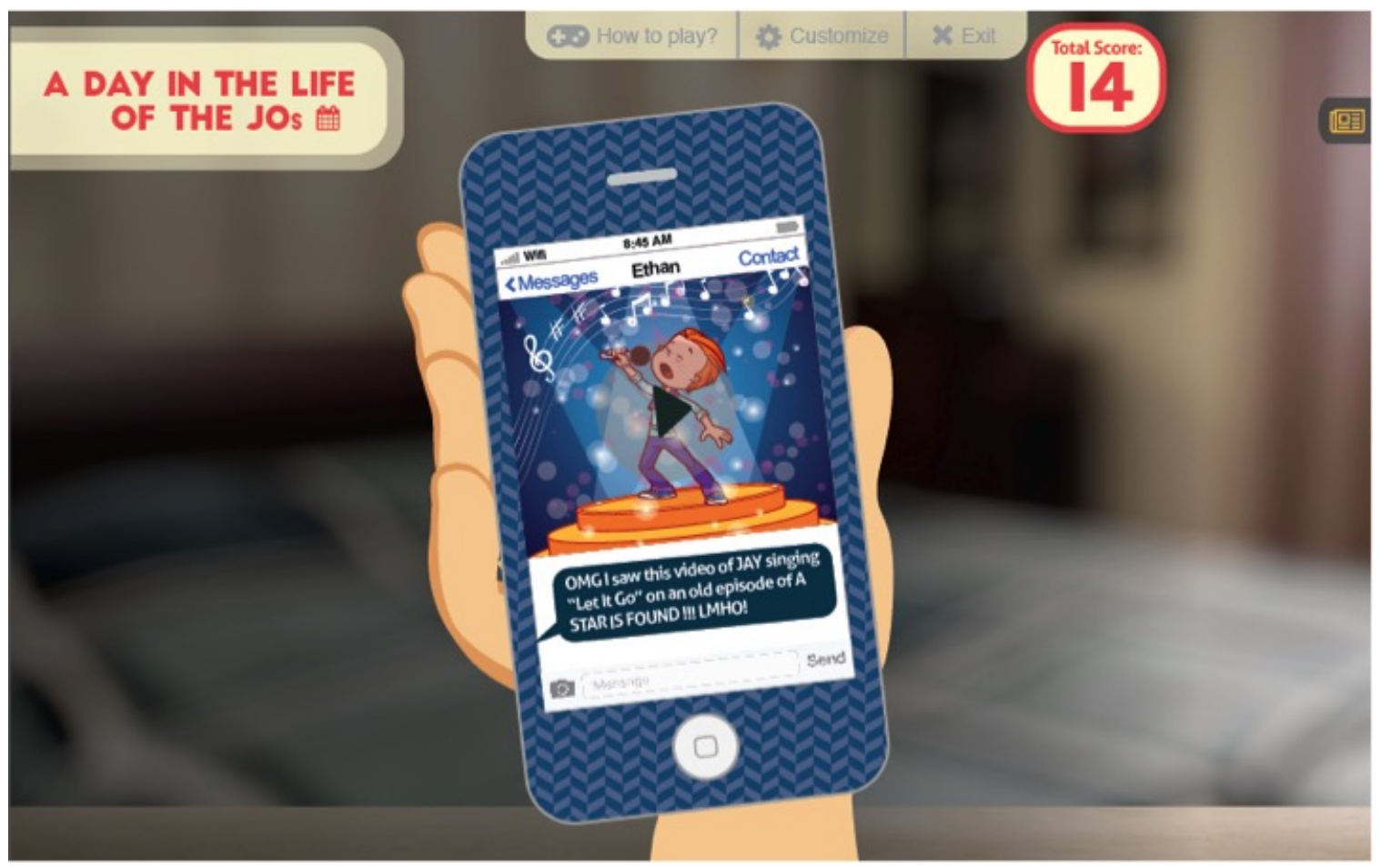

Figure A.70: D4-Image sharing: Scene 2 of fifth scenario.

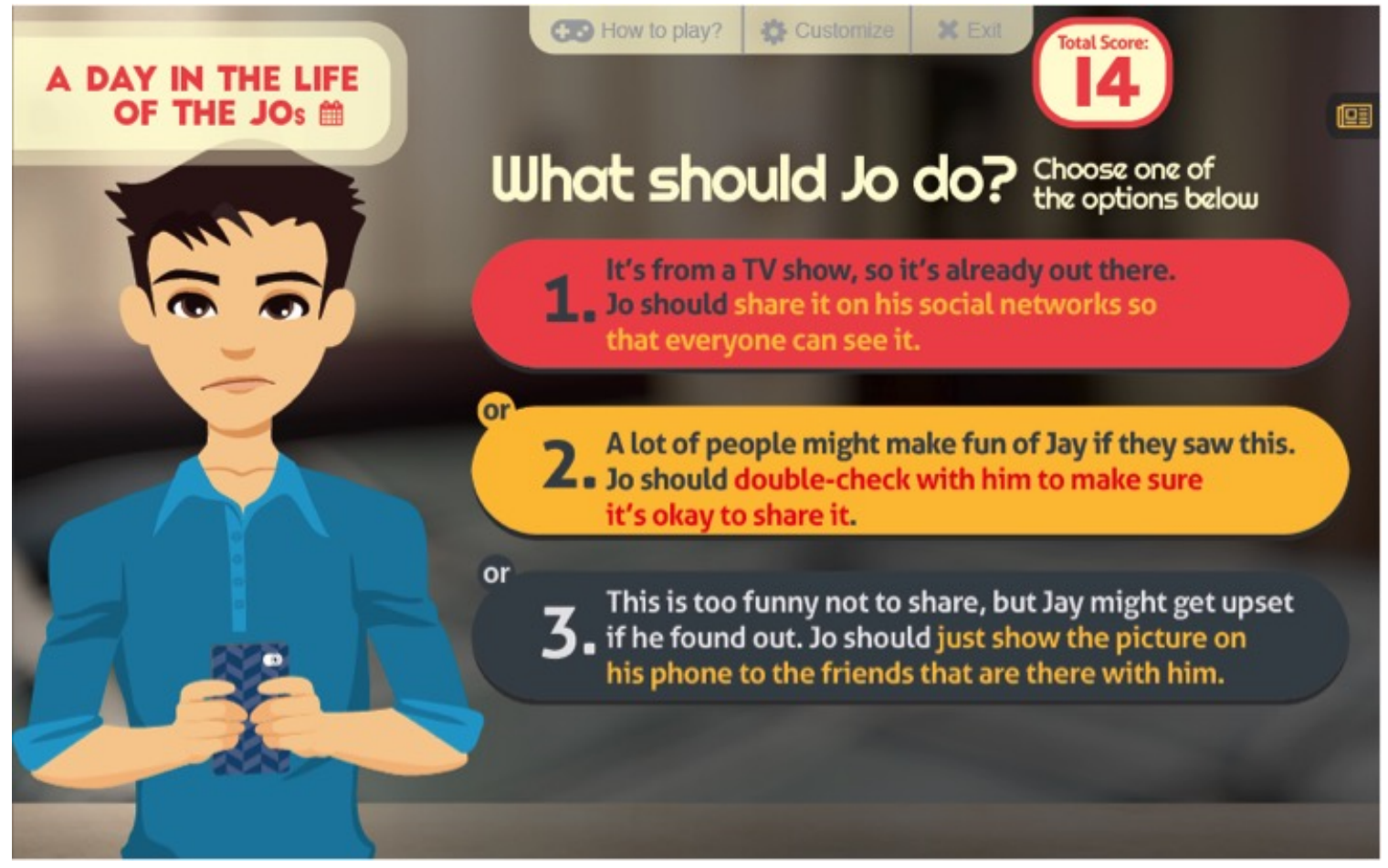

Figure A.71: D4-Image sharing: Scene 3 of fifth scenario. 


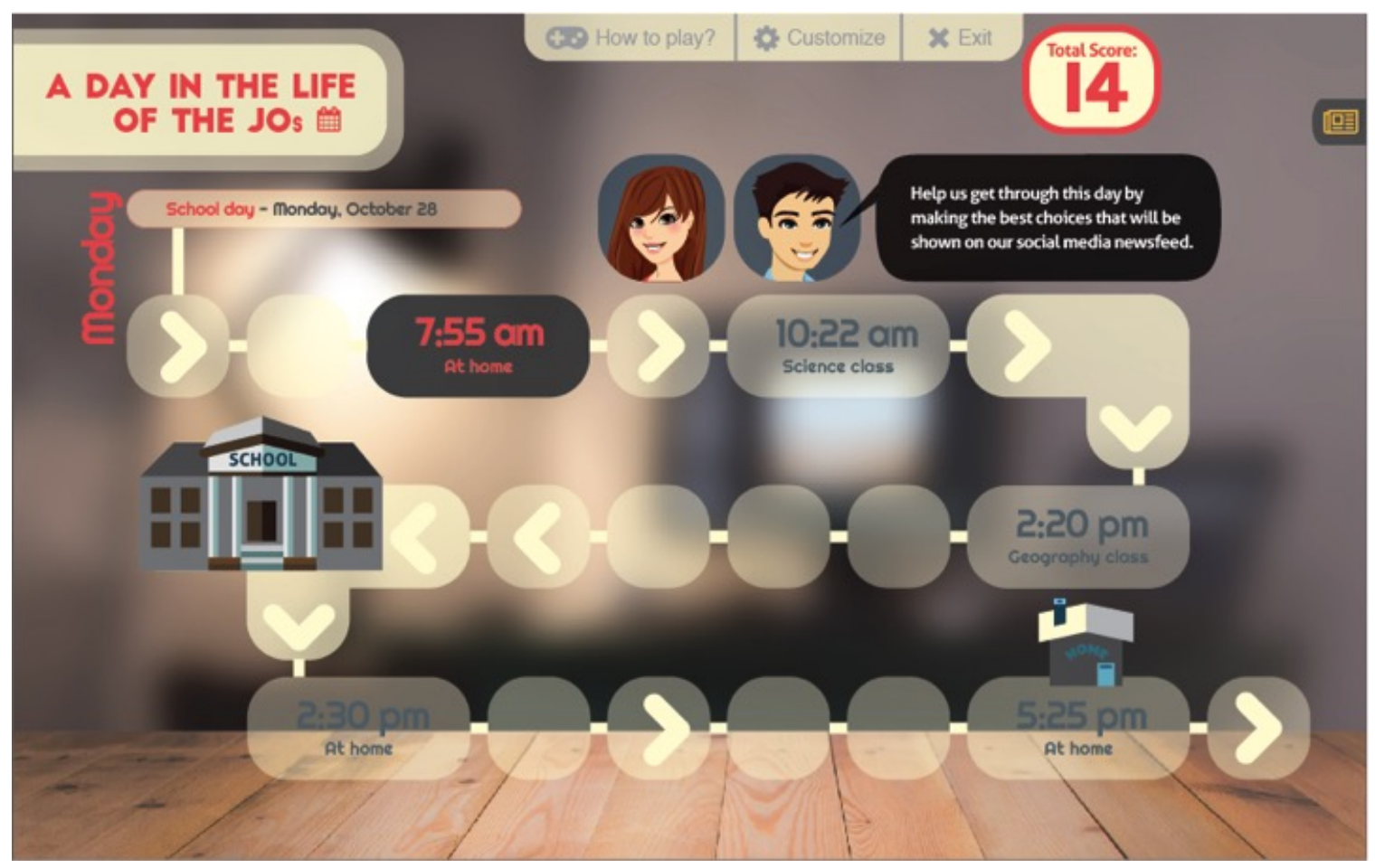

Figure A.72: The Day View of D5-Verification of information from online sources topic. 


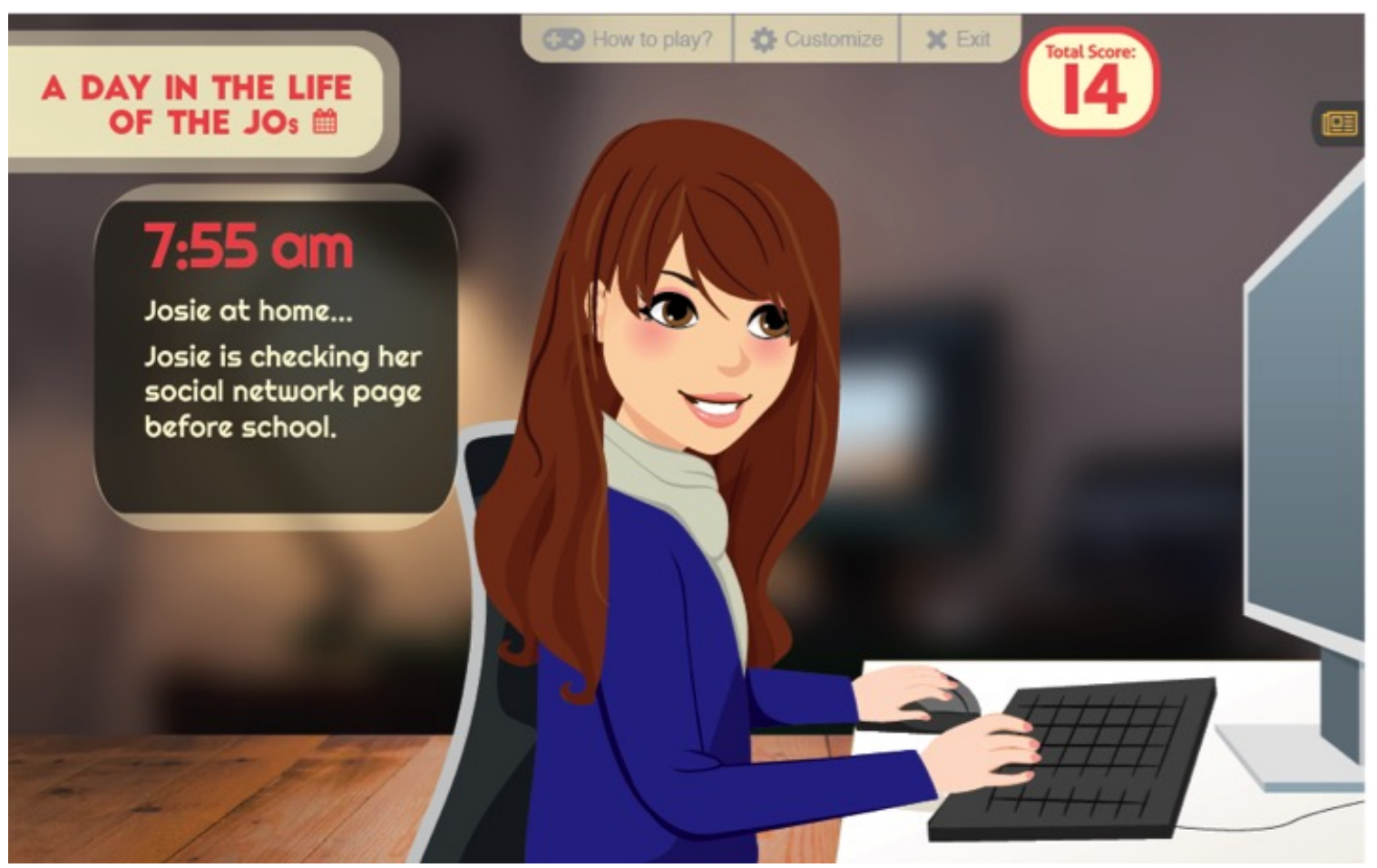

Figure A.73: D5-Verification of information from online sources: Scene 1 of first scenario. 


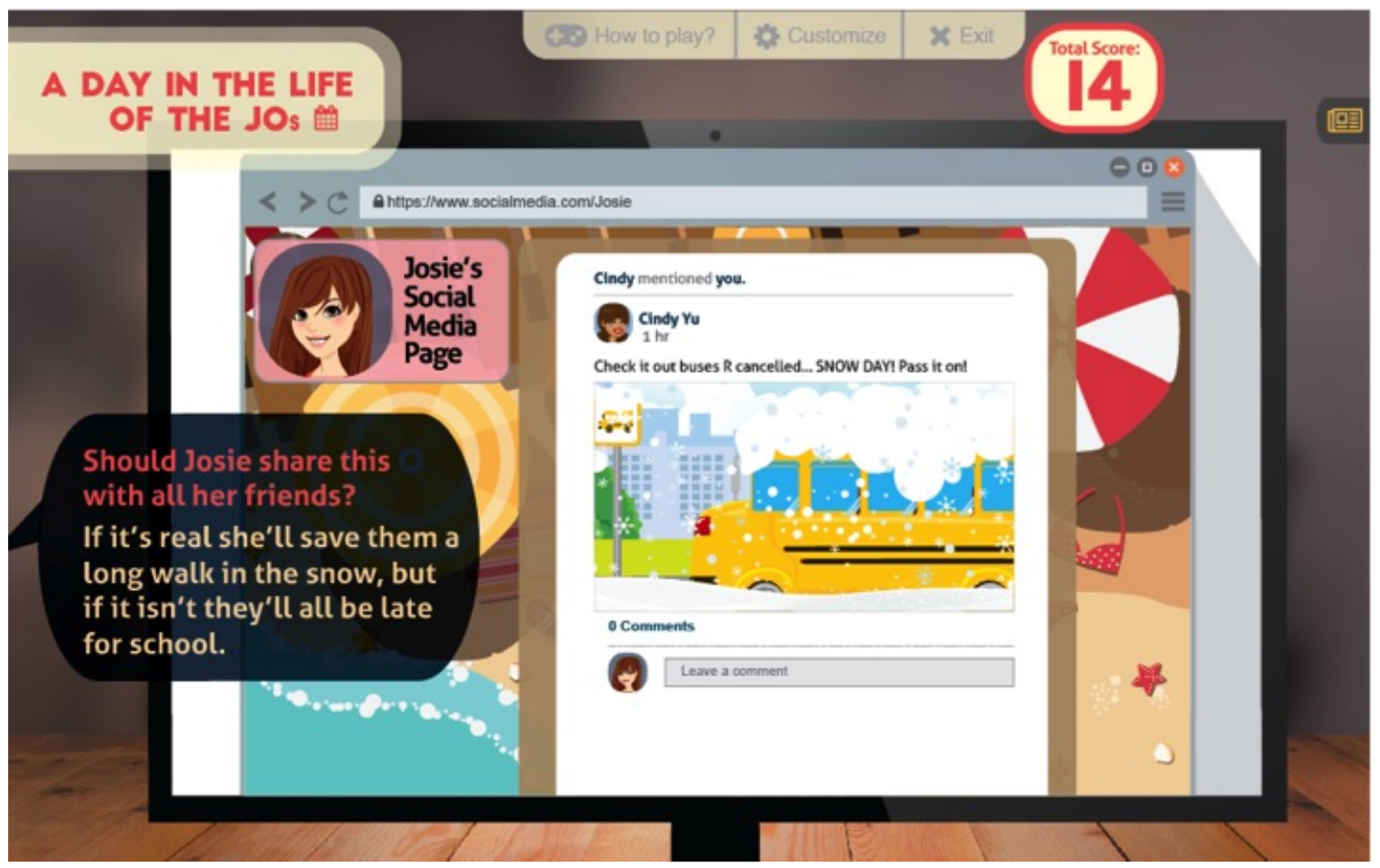

Figure A.74: D5-Verification of information from online sources: Scene 2 of first scenario. 


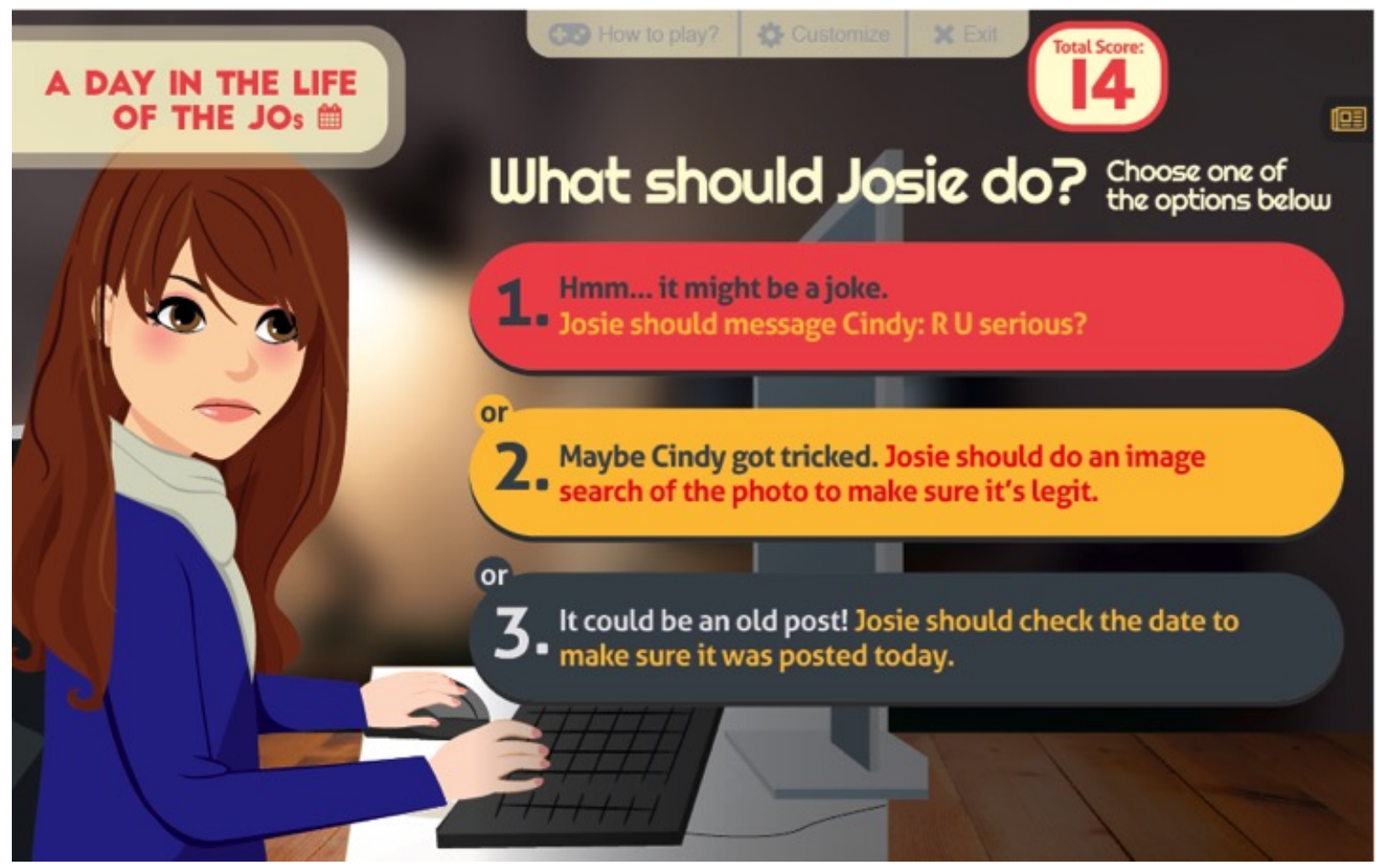

Figure A.75: D5-Verification of information from online sources: Scene 3 of first scenario. 


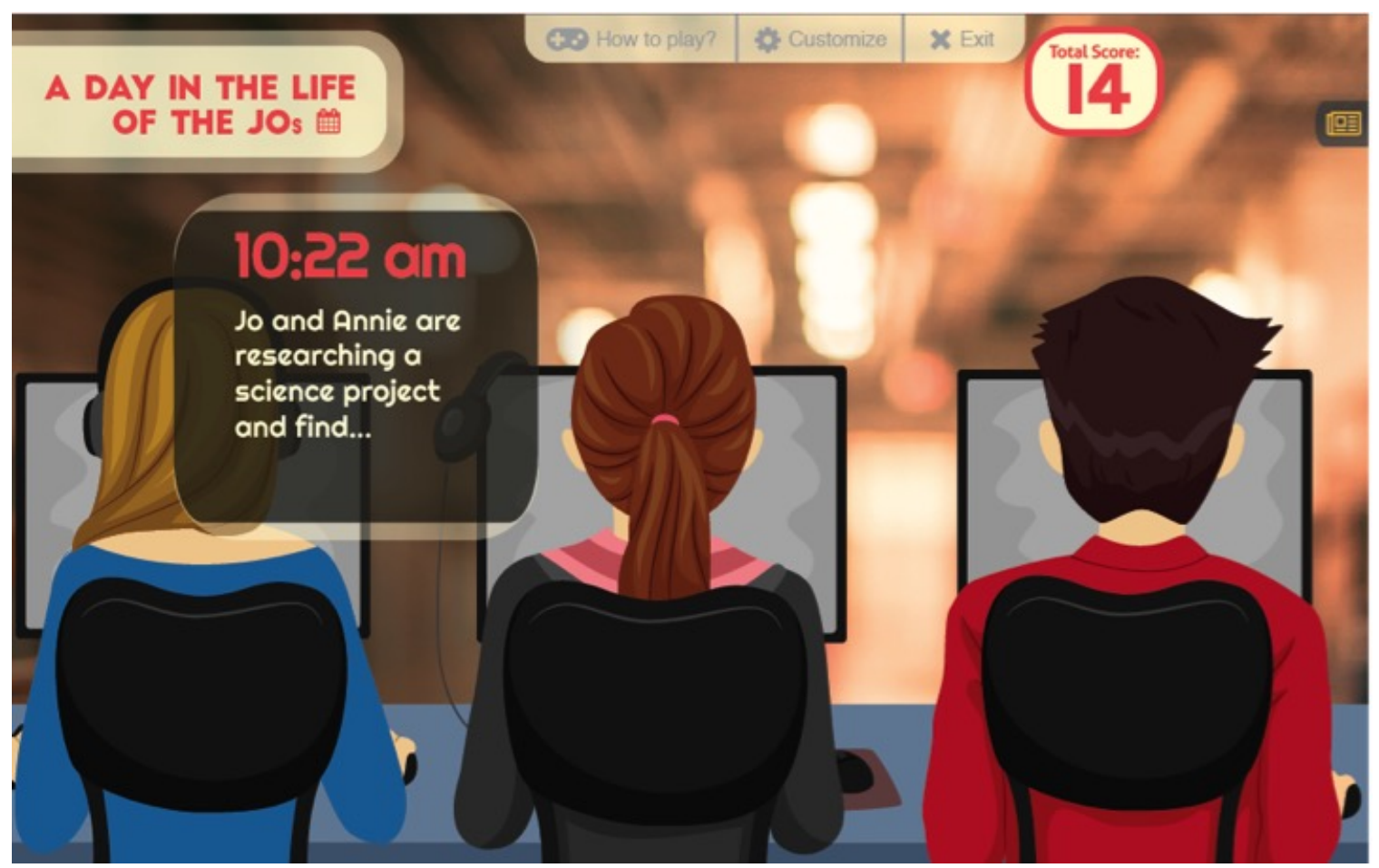

Figure A.76: D5-Verification of information from online sources: Scene 1 of second scenario. 


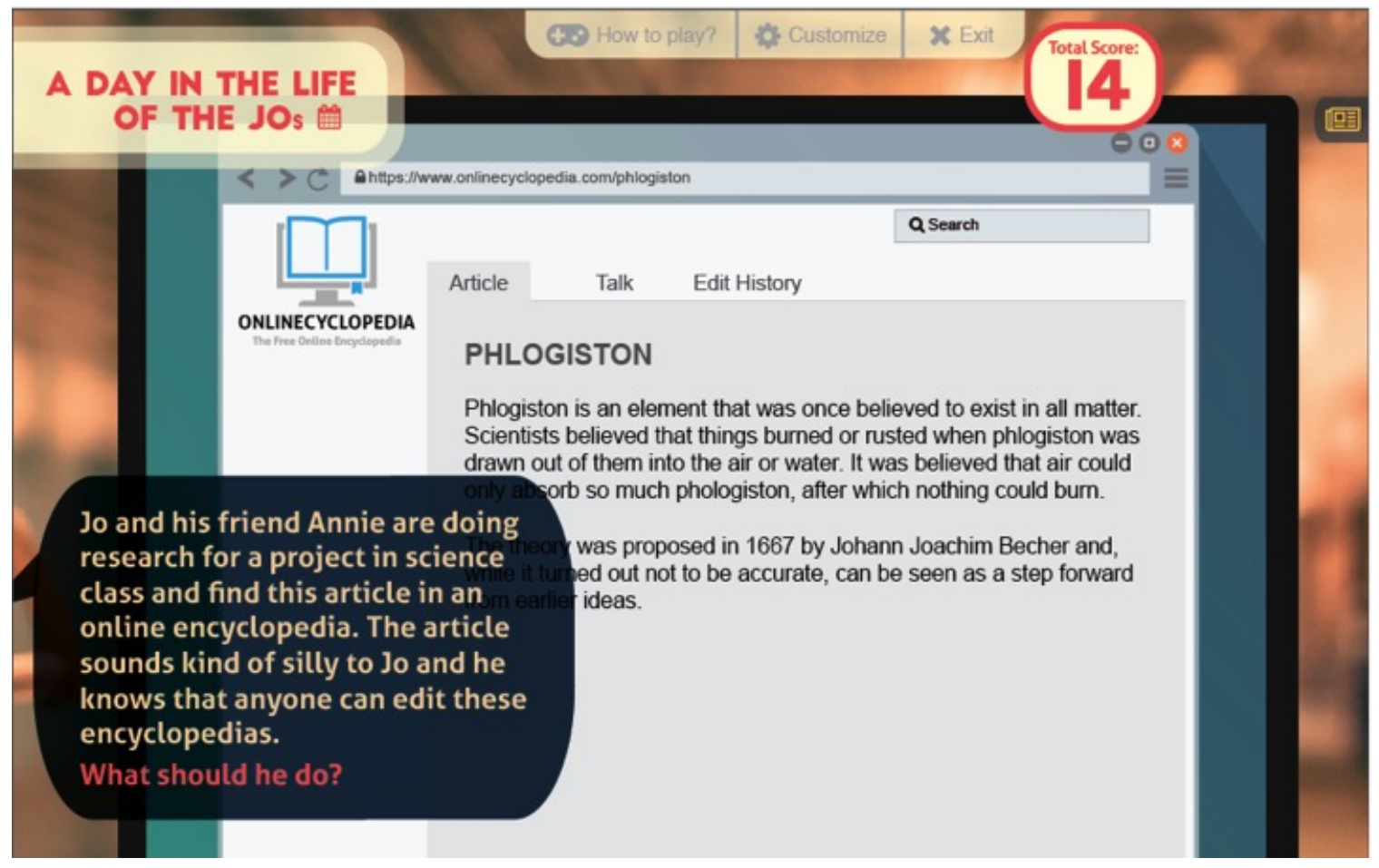

Figure A.77: D5-Verification of information from online sources: Scene 2 of second scenario. 


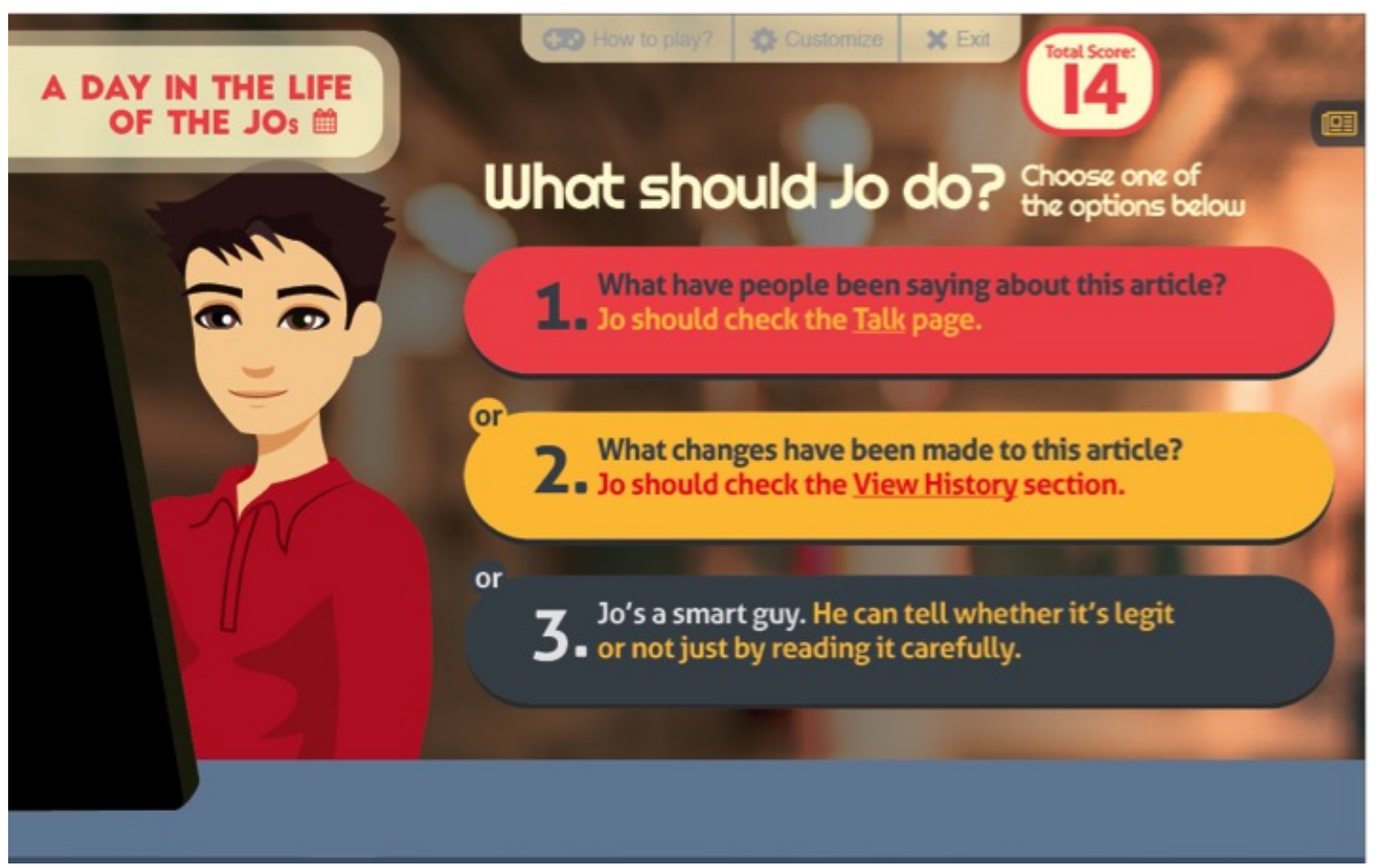

Figure A.78: D5-Verification of information from online sources: Scene 3 of second scenario. 


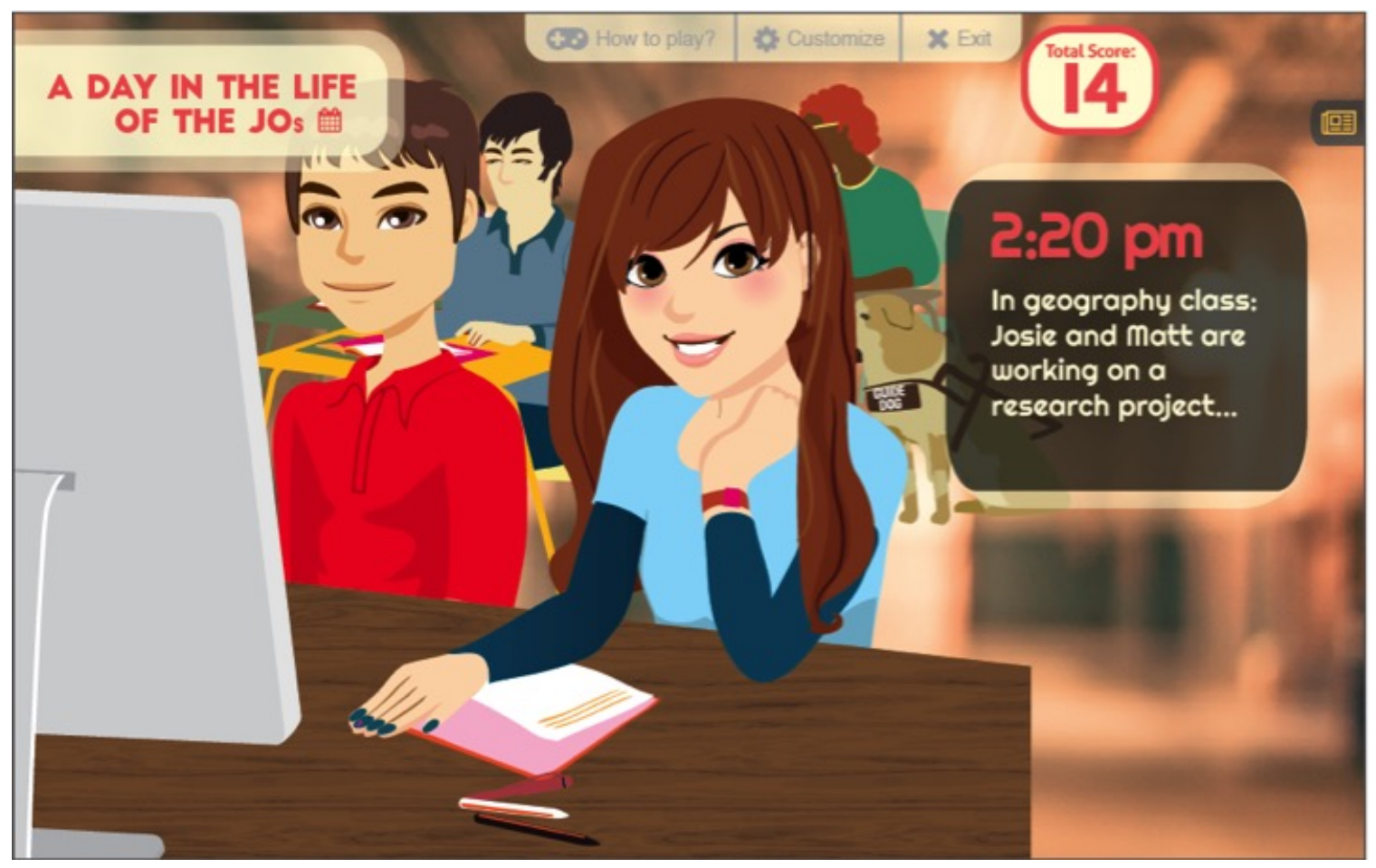

Figure A.79: D5-Verification of information from online sources: Scene 1 of third scenario. 


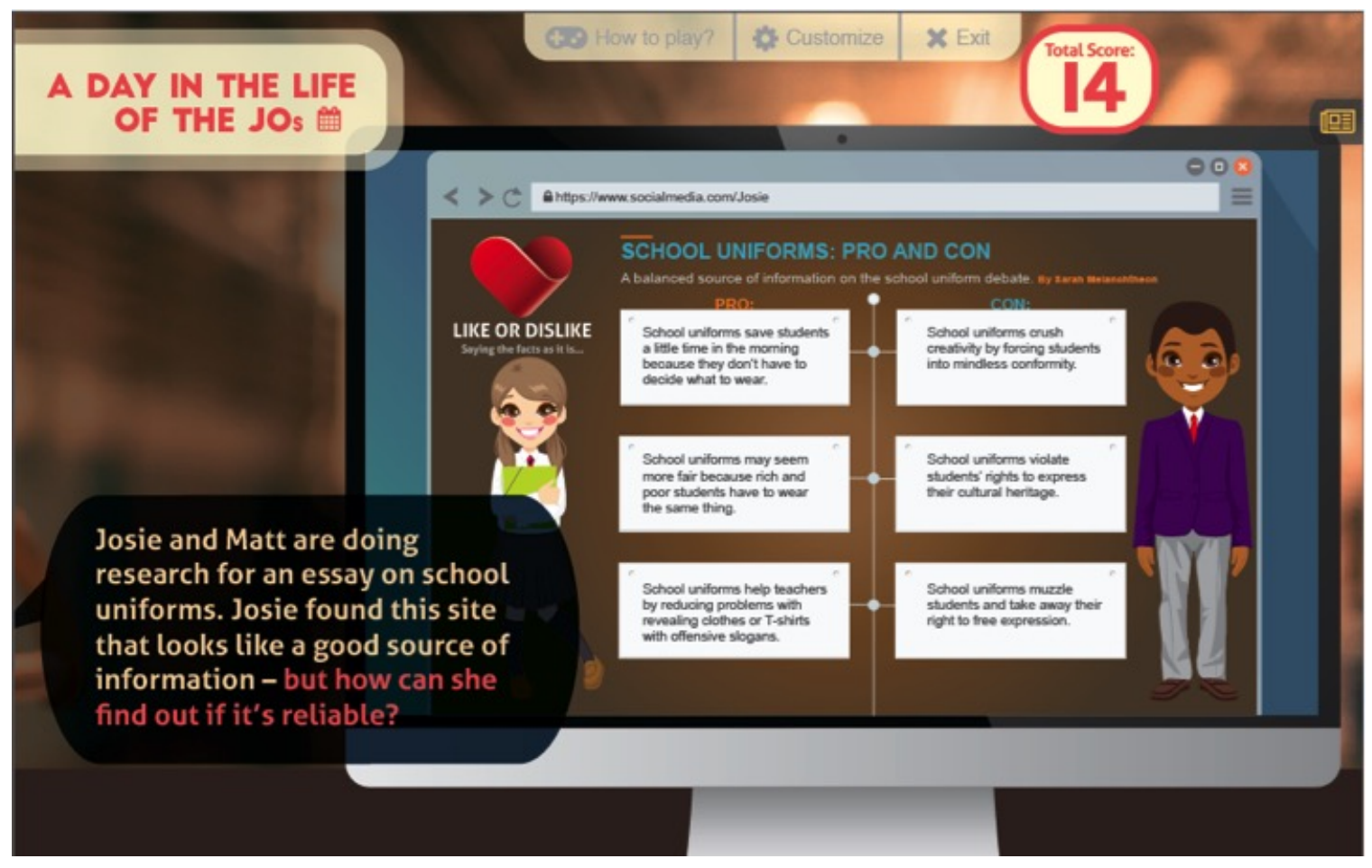

Figure A.80: D5-Verification of information from online sources: Scene 2 of third scenario. 


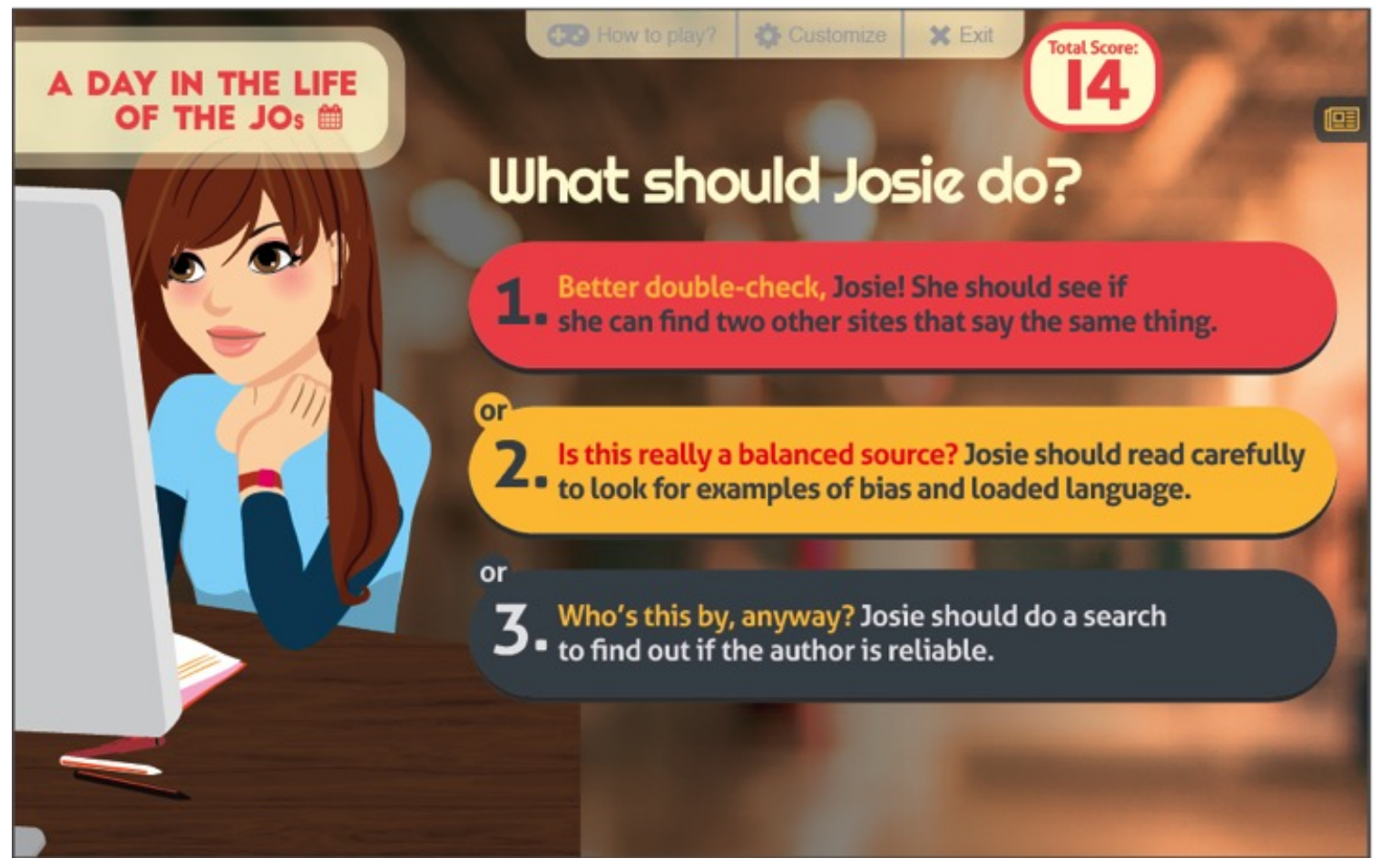

Figure A.81: D5-Verification of information from online sources: Scene 3 of third scenario. 


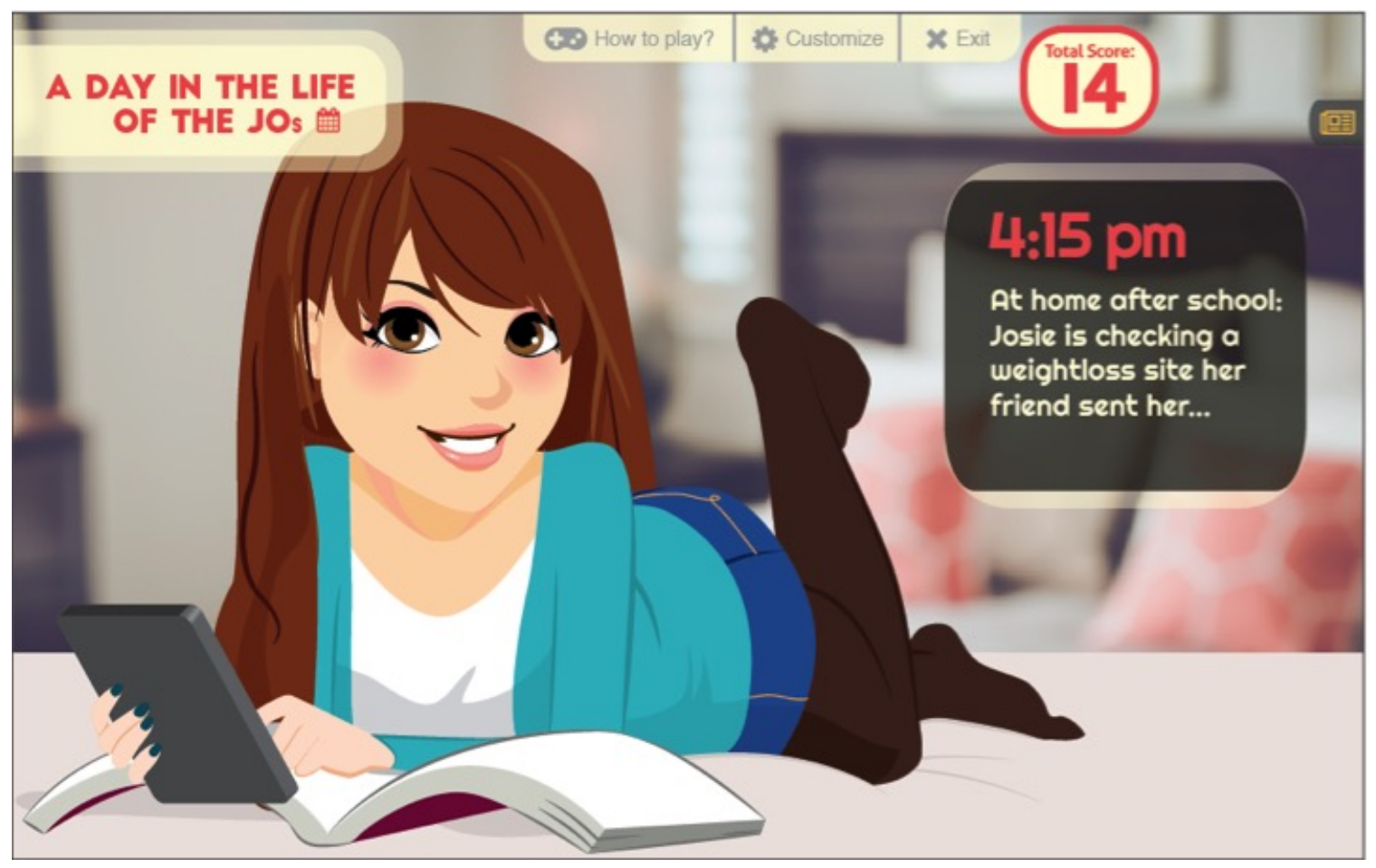

Figure A.82: D5-Verification of information from online sources: Scene 1 of fourth scenario. 


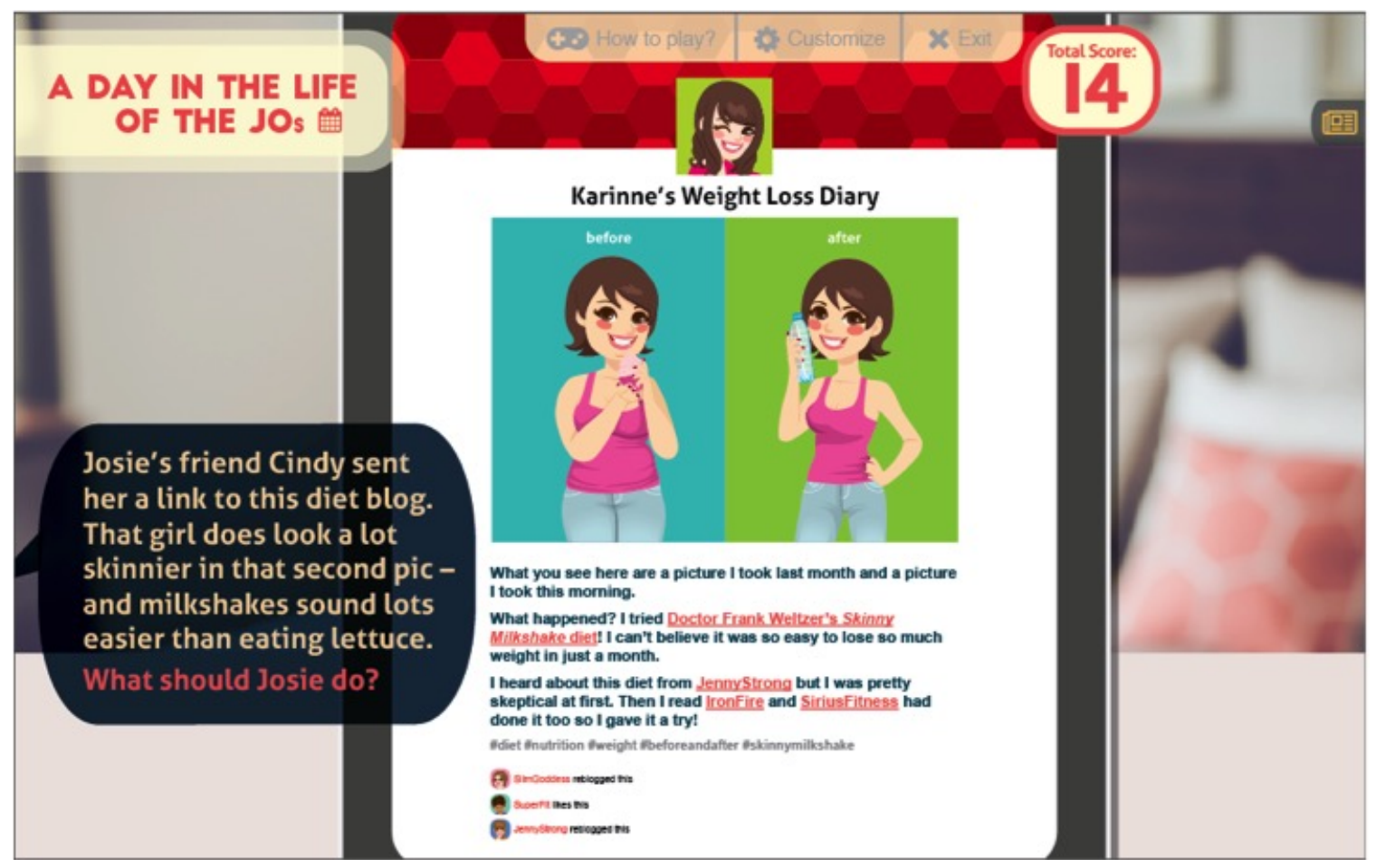

Figure A.83: D5-Verification of information from online sources: Scene 2 of fourth scenario. 


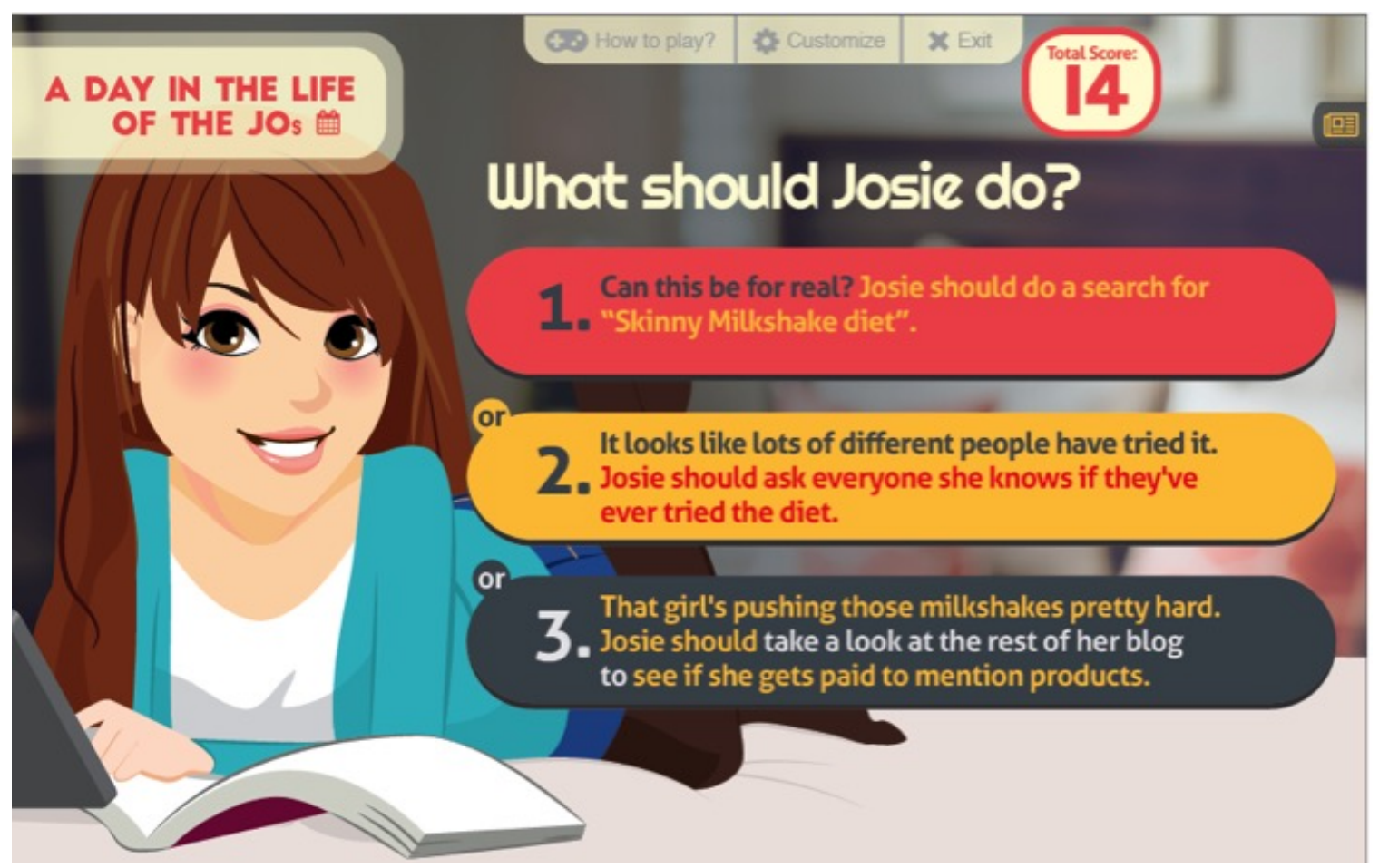

Figure A.84: D5-Verification of information from online sources: Scene 3 of fourth scenario. 


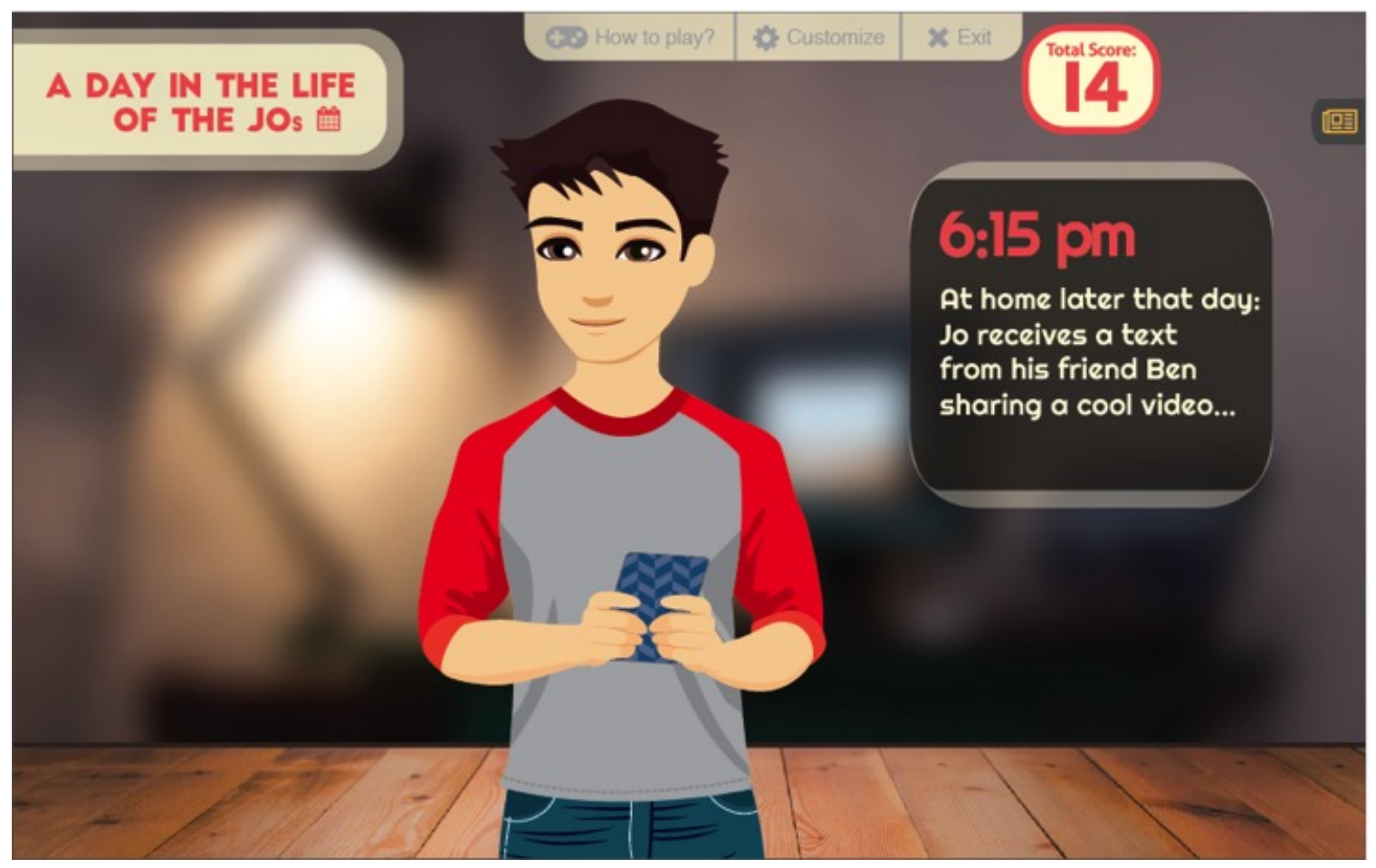

Figure A.85: D5-Verification of information from online sources: Scene 1 of fifth scenario. 


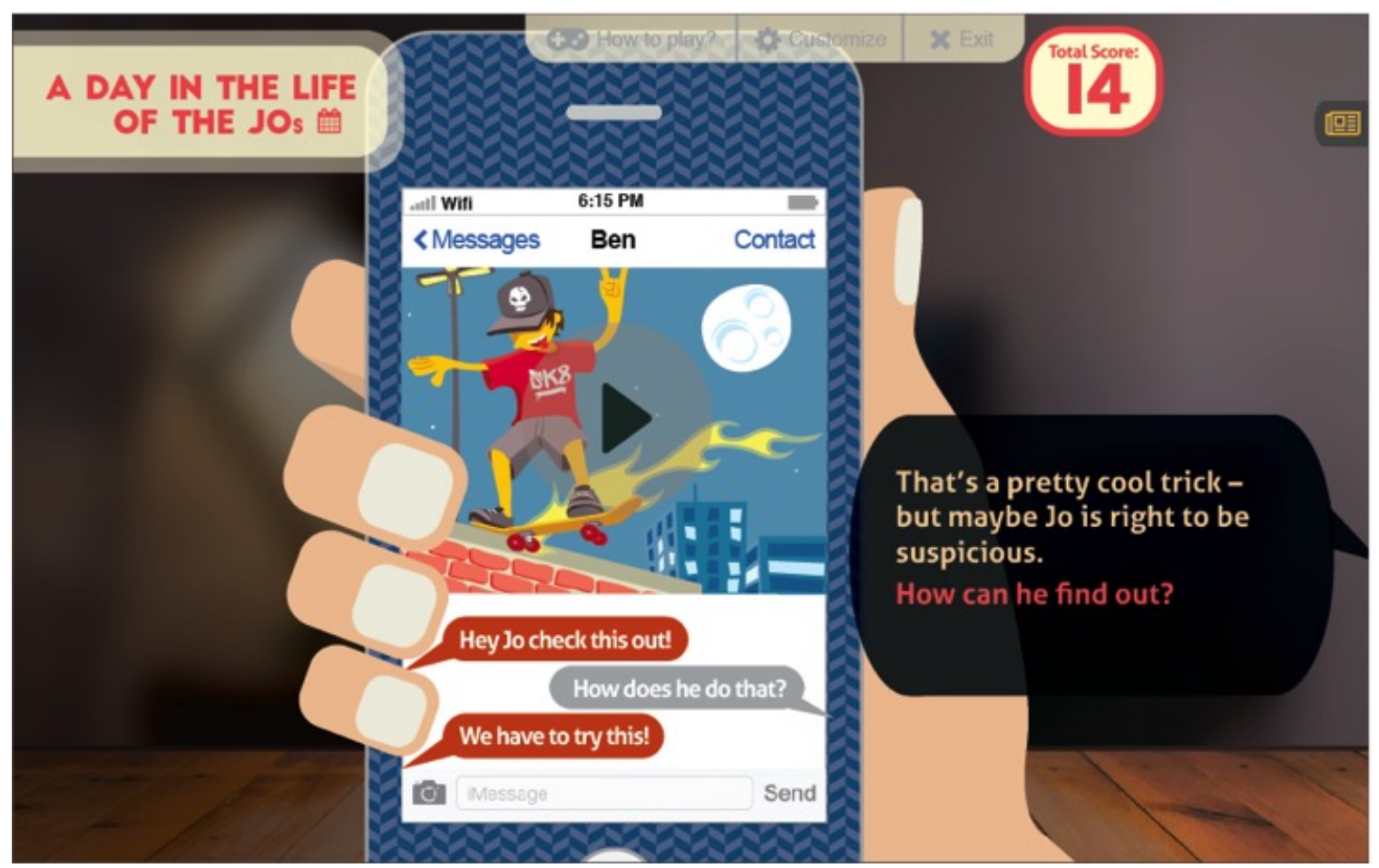

Figure A.86: D5-Verification of information from online sources: Scene 2 of fifth scenario. 


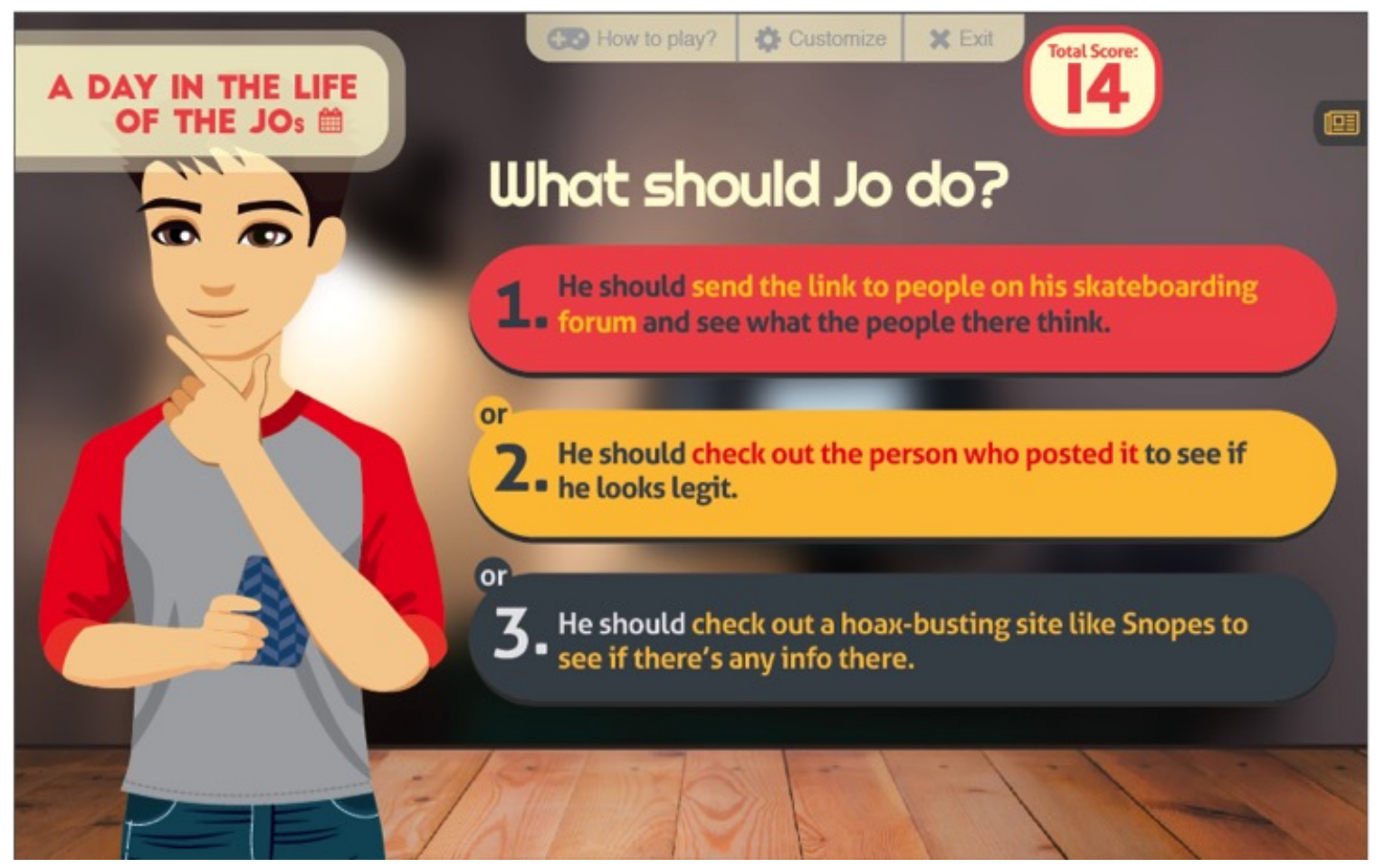

Figure A.87: D5-Verification of information from online sources: Scene 3 of fifth scenario. 